\title{
Boolean-Valued Set-Theoretic Systems: General Formalism and Basic Technique
}

\author{
Alexander Gutman ${ }^{1,2}$ (D) \\ 1 Laboratory of Functional Analysis, Sobolev Institute of Mathematics, 630090 Novosibirsk, Russia; \\ gutman@math.nsc.ru or a.e.gutman@gmail.com; Tel.: +7-913-067-4848 \\ 2 Department of Physics and Mathematics, Novosibirsk State University, 630090 Novosibirsk, Russia
}

Citation: Gutman, A.

Boolean-Valued Set-Theoretic Systems: General Formalism and Basic Technique. Mathematics 2021, 9 , 1056. https://doi.org/10.3390/math 9091056

Academic Editor: Anatoly

Georgievich Kusraev

Received: 4 April 2021

Accepted: 23 April 2021

Published: 8 May 2021

Publisher's Note: MDPI stays neutral with regard to jurisdictional claims in published maps and institutional affiliations.

Copyright: (C) 2021 by the author. Licensee MDPI, Basel, Switzerland. This article is an open access article distributed under the terms and conditions of the Creative Commons Attribution (CC BY) license (https:// creativecommons.org/licenses/by/ $4.0 /)$.

\begin{abstract}
This article is devoted to the study of the Boolean-valued universe as an algebraic system. We start with the logical backgrounds of the notion and present the formalism of extending the syntax of Boolean truth values by the use of definable symbols, internal classes, outer terms and external Boolean-valued classes. Next, we enrich the collection of Boolean-valued research tools with the technique of partial elements and the corresponding joins, mixings and ascents. Passing on to the set-theoretic signature, we prove that bounded formulas are absolute for transitive Boolean-valued subsystems. We also introduce and study intensional, predicative, cyclic and regular Boolean-valued systems, examine the maximum principle, and analyze its relationship with the ascent and mixing principles. The main applications relate to the universe over an arbitrary extensional Booleanvalued system. A close interrelation is established between such a universe and the intensional hierarchy. We prove the existence and uniqueness of the Boolean-valued universe up to a unique isomorphism and show that the conditions in the corresponding axiomatic characterization are logically independent. We also describe the structure of the universe by means of several cumulative hierarchies. Another application, based on the quantifier hierarchy of formulas, improves the transfer principle for the canonical embedding in the Boolean-valued universe.
\end{abstract}

Keywords: Boolean-valued universe; algebraic system; set theory; cumulative hierarchy

MSC: 03C90; 03E40

\section{Introduction}

This article is devoted to the study of Boolean-valued algebraic systems of set-theoretic signature. The primary audience is assumed to be general mathematicians who are interested in the formal backgrounds of Boolean-valued analysis.

The key facts presented here are not new: The defining axioms of a Boolean-valued universe, its existence, uniqueness, and basic properties (such as the transfer, ascent, mixing and maximum principles) are well known. What is new here is a systematic study of the Boolean-valued universe as an algebraic system, with some new tools including partial elements, superstructures over extensional Boolean-valued systems, and intensional cumulative hierarchies.

The main content of the paper is divided into four sections. Section 2, "General Formalism," is devoted to the notion of Boolean-valued algebraic system and exposes the logical backgrounds of various useful extensions of the syntax of Boolean truth values. In Section 3, "Basic Technique," we present the main tools related to Boolean-valued systems (including the new apparatus of partial elements), study the key properties of the systems, and clarify interrelations between them. In Section 4, "The Structure of the Boolean-Valued Universe," we elaborate the notion of universe over an arbitrary extensional Boolean-valued system and present the main results on the classical universe $\mathbb{V}^{(B)}$ : existence, uniqueness, logical independence of the axioms, hierarchical structure. In Section 5, "Applications of the Lévy Hierarchy," we suggest a development of the technique based on the quantifier 
classification of formulas and demonstrate how it can improve the Boolean-valued transfer principle for the canonical embedding.

Let $\mathbb{F}$ be the totality of all formulas of set-theoretic signature $\{=, \in\}$ defined in the metatheory. The metaformula that defines $\mathbb{F}$ can be rendered into the language of ZFC as a formula, $\delta_{\mathbb{F}}(x) \in \mathbb{F}$, thus providing a ZFC definition, $x=\ulcorner\mathbb{F}\urcorner \Leftrightarrow \delta_{\mathbb{F}}(x)$, for the set $\ulcorner\mathbb{F}\urcorner$ of internal formulas. The same is true of the metaset $\mathbb{S} \subset \mathbb{F}$ of sentences (i.e., formulas without free variables) and the set $\ulcorner\mathbb{S}\urcorner$ of internal sentences. In a similar manner, given an arbitrary formula $\varphi \in \mathbb{F}$, we may render $\varphi$ into its description $\delta_{\varphi}(x) \in \mathbb{F}$ and thus obtain a ZFC definition, $x=\ulcorner\varphi\urcorner \Leftrightarrow \delta_{\varphi}(x)$, for the set $\ulcorner\varphi\urcorner$, the code of $\varphi$. This results in a conservative extension (actually, an eliminable extension; see Definition 5) of ZFC by means of the definable constants $\ulcorner\mathbb{F}\urcorner,\ulcorner\mathbb{S}\urcorner$, and $\ulcorner\varphi\urcorner$ such that $\mathrm{ZFC} \vdash(\ulcorner\varphi\urcorner \in\ulcorner\mathbb{F}\urcorner,\ulcorner\sigma\urcorner \in\ulcorner\mathbb{S}\urcorner)$ for all $\varphi \in \mathbb{F}, \sigma \in \mathbb{S}$.

In all contemporary articles and textbooks devoted to Boolean-valued models of set theory, given a complete Boolean algebra $B$, the truth valuation in the $B$-valued universe $\mathbb{V}^{(B)}$ is described informally either as a class function

$$
\llbracket \cdot \rrbracket: \sigma \in \mathbb{S}\left(\mathbb{V}^{(B)}\right) \mapsto \llbracket \sigma \rrbracket \in B
$$

mapping each sentence $\sigma$ of signature $\{=, \in\} \cup \mathbb{V}^{(B)}$, with elements of $\mathbb{V}^{(B)}$ regarded as constants, to an element $\llbracket \sigma \rrbracket$ of $B$; or as a class function

$$
\llbracket \cdot \rrbracket:\left(\varphi, x_{1}, \ldots, x_{n}\right) \in \bigcup_{m \in \omega} \mathbb{F}_{m} \times\left(\mathbb{V}^{(B)}\right)^{m} \mapsto \llbracket \varphi\left(x_{1}, \ldots, x_{n}\right) \rrbracket \in B
$$

that maps each pair, constituted by a formula $\varphi \in \mathbb{F}$ with $n$ free variables and a tuple $\left(x_{1}, \ldots, x_{n}\right) \in\left(\mathbb{V}^{(B)}\right)^{n}$, to an element $\llbracket \varphi\left(x_{1}, \ldots, x_{n}\right) \rrbracket$ of $B$; see, for example, [1] (3.1-3.3), [2] (Boolean-Valued Models, pp. 206-207), [3] (Construction of the model, pp. 20-29), [4] $(2.1 .6,2.1 .7),[5](4.1 .6,4.1 .7)$. The informality mentioned above is twofold. First, the set $\ulcorner\mathbb{F}\urcorner$ of internal formulas is implicitly meant instead of $\mathbb{F}$; and, second, the class function $\llbracket \cdot \rrbracket$ is actually not defined and, moreover, cannot be defined. The latter is explained as follows: In the case of $B=2:=\{0,1\}$, the separated 2-valued universe $\mathbb{V}^{(2)}$ is naturally isomorphic to $\mathbb{V}$, and the truth function $\llbracket \cdot \rrbracket:\ulcorner\mathbb{S}\urcorner \rightarrow\{0,1\}$ satisfies the condition

$$
\mathrm{ZFC} \vdash(\sigma \Leftrightarrow \mathbb{V} \vDash\ulcorner\sigma\urcorner \Leftrightarrow \llbracket\ulcorner\sigma\urcorner \rrbracket=1),
$$

for all sentences $\sigma \in \mathbb{S}$. Therefore, if $\llbracket \cdot \rrbracket$ were definable, the formula $\tau(s):=(\llbracket s \rrbracket=1)$ would be a truth predicate for ZFC:

$$
\mathrm{ZFC} \vdash(\sigma \Leftrightarrow \tau(\ulcorner\sigma\urcorner)), \quad \sigma \in \mathbb{S} ;
$$

which, by Tarski's undefinability theorem [2] (12.7), is impossible unless ZFC is inconsistent.

The authors are certainly aware of the informality. For instance, there is a warning in [2] (Models of Set Theory and Relativization, pp. 161-162) that the relativization $M \vDash \varphi$ is not defined for $\varphi \in\ulcorner\mathbb{F}\urcorner$, and the satisfaction $M \vDash\ulcorner\varphi\urcorner$ is not defined if $M$ is a proper class; and when considering models of set theory that are proper classes, due to Gödel's Second Incompleteness Theorem, we have to be careful how the generalization is formulated. In [3] (Construction of the model, Remark 1, p. 24) this "tiresome point" is commented as follows: "The construction of $\llbracket \sigma \rrbracket$ for arbitrary $\sigma$ evidently has the form of a truth definition for set theory and so cannot be completely formalized within the language of set theory... The machinery available in ZFC is not (unless ZFC is inconsistent) strong enough to formalize the construction of the map $\sigma \mapsto \llbracket \sigma \rrbracket$ as a function of $\sigma$. More precisely, one can prove in ZFC that the collection of all pairs $(\sigma, \llbracket \sigma \rrbracket)$ is not a definable class. We must therefore think of this map as being defined metalinguistically." Furthermore, as was said in [6], because of the "undefinability of truth" one cannot express " $\psi(\varphi)$ holds in $\mathbb{V}^{(B)}$ for all formulas $\varphi^{\prime \prime}$ in a single set-theoretical formula. Usually what is in question is a scheme of theorems and there is no particular difficulty giving a correct treatment. People are sloppy about this detail but that is only to concentrate on the essentials. 
In the present article, we do not avoid inessentials and do our best to give a correct treatment of all the details. We discard internal formulas, define the Boolean truth valuation "metalinguistically," and thoroughly expose the logical backgrounds of Boolean-valued modeling. This is what Section 2, "General Formalism," is devoted to. In the section, we provide an accurate formal definition for the notion of Boolean-valued algebraic system and also justify the use of theoretically definable symbols, internal classes, outer terms, and external Boolean-valued classes in the truth valuation syntax.

Another subject currently lacking in the literature is the study of $\mathbb{V}^{(B)}$ as an algebraic system of set-theoretic signature. General properties of Boolean-valued systems were considered only in their connection with representation in $\mathbb{V}^{(B)}$ and with specific technical aspects of ascents and descents; see [4] (Chapter 4), [5] (Chapter 7). Until recently, Reference [1] was the only publication in which the characteristic algebraic properties of $\mathbb{V}^{(B)}$ were listed; see [1] (3.4). In Section 3, "Basic Technique," we methodically examine those key properties of Boolean-valued algebraic systems under the names of extensionality, regularity, intensionality, and predicativity. The main tools in this research include the new apparatus of partial elements, joins of antichains, mixings of subclasses, ascents and descents of various kinds, the use of Boolean-valued classes in the language of truth values, and the absoluteness of bounded formulas for transitive Boolean-valued subsystems. We also introduce and study $\sigma$-regular Boolean-valued systems, examine the maximum principle, and analyze its relationship with the ascent and mixing principles.

The axiomatic characterization of the Boolean-valued universe presented in [1] (3.4) became the main motivation for our research; and the primary aim was to develop approaches to proving the uniqueness assertion claimed therein (see Definition 55 and Theorem 15). Furthermore, Professor Robert M. Solovay noted in [6] that all the axioms listed in [1] (3.4) were needed for a complete description of the $B$-valued universe, and the examples for this could be given in the special case of $B=\{0,1\}$. For instance, to justify the necessity of regularity, one could build up a universe not from the empty set but from a non-well-founded collection. Therefore, we aimed at proving that the five conditions (a)-(e) of Definition 55 listed in the axiomatic characterization of $\mathbb{V}^{(B)}$ are logically independent, and, moreover, we aimed to do that for all complete Boolean algebras $B$. To this end, we elaborated the notion of universe over an arbitrary extensional Boolean-valued system and established a close interrelation between such a universe and the intensional hierarchy, a Boolean-valued analog of the von Neumann cumulative hierarchy. This general tool, presented in Section 4, "The Structure of the Boolean-Valued Universe," makes our aims easily achievable (see Theorem 15 and Examples 1-5). As a bonus, we obtain the descriptions of $\mathbb{V}^{(B)}$ by means of four cumulative hierarchies (see Section 4.3).

Another bonus can be derived from the formalism of eliminable extensions exposed in Section 2.2. As soon as we know the logical backgrounds of formal definitions, we can analyze the structure of the low-level set-theoretic translations of definable mathematical objects and properties. In certain cases, this knowledge can considerably simplify verification of the validity of complex assertions inside the Boolean-valued universe. As is known (see [4] (2.2.9), [5] (4.2.9)), if an assertion $\varphi\left(x_{1}, \ldots, x_{n}\right)$ about sets $x_{1}, \ldots, x_{n}$ belongs to class $\Sigma_{1}$, that is, can be expressed by a set-theoretic formula

$$
\left(\exists y_{1}\right) \ldots\left(\exists y_{m}\right) \psi\left(x_{1}, \ldots, x_{n}, y_{1}, \ldots, y_{m}\right),
$$

with all quantifiers in $\psi$ having the form $(\forall u \in v)$ or $(\exists u \in v)$; then $\varphi\left(x_{1}, \ldots, x_{n}\right)$ implies the validity of $\varphi\left(x_{1}^{\wedge}, \ldots, x_{n}^{\wedge}\right)$ inside $\mathbb{V}^{(B)}$ for every complete Boolean algebra $B$. In Section 5 , "Applications of the Lévy Hierarchy", we suggest some additions to the set of tools which help to successively build more and more complex formulas and terms, while staying within the class of $\Sigma_{1}$ constructions. As an example, we demonstrate that the use of the tools can shorten the proof of the validity $\mathbb{V}^{(B)} \vDash\left(\mathcal{P}_{\text {fin }}(X)^{\wedge}=\mathcal{P}_{\text {fin }}\left(X^{\wedge}\right)\right)$ from a couple of pages to a couple of lines (see Section 5.4). We also analyze the logical structure of several classical definitions of the field of reals and find out which of them guarantee the inclusion $\mathbb{R}^{\wedge} \subset \mathbb{R}$ inside $\mathbb{V}^{(B)}$ for all $B$. 


\section{General Formalism}

Since the primary audience is not assumed to consist of specialists in logic or formal languages, we consider it appropriate to start the exposition with describing the logical machinery of formal definitions, utilization of classes in set theory, and the use of infinite assertions in implications. In this section, we present the basic information related to the notion of Boolean-valued algebraic system and formalize the use of definable symbols, outer terms and external Boolean-valued classes in the syntax of Boolean truth values.

\subsection{Logical Prerequisites}

As a logical base we use the classical Hilbert-style first-order predicate calculus with equality. Therefore, throughout the article, we assume that, first, all signatures under consideration contain the binary predicate symbol "=" and, second, the axioms of the calculus include the standard axioms of equality.

Definition 1. Let $\Sigma$ be a signature. (The signature can be infinite but is always assumed at most countable and decidable.) By a theory (more exactly, an axiomatizable theory) of signature $\Sigma$ we mean an arbitrary decidable subset $\mathcal{T}$ of the set $F(\Sigma)$ of formulas of signature $\Sigma$. The elements of $\mathcal{T}$ are called the special axioms (or nonlogical axioms) of the theory. Given a formula $\varphi \in F(\Sigma)$, the expression $\mathcal{T} \vdash \varphi$ means that $\varphi$ is a theorem of $\mathcal{T}$, that is, $\varphi$ is deducible from the axioms of the predicate calculus of signature $\Sigma$ with equality and the special axioms of $\mathcal{T}$ by means of the classical deduction rules. If $\mathcal{F}$ is a set of formulas, we write $\mathcal{T} \vdash \mathcal{F}$ whenever $\mathcal{T} \vdash \varphi$ for all $\varphi \in \mathcal{F}$. The expression $\vdash \varphi$ serves as a shorthand for $\varnothing \vdash \varphi$ and thus means that $\varphi$ is provable in the calculus of signature $\Sigma$ without any special axioms. Due to the Soundness and Completeness theorems, a formula $\varphi$ meets $\vdash \varphi$ if and only if $\varphi$ is a tautology of signature $\Sigma$, that is, $\varphi$ is true in every algebraic system of signature $\Sigma$ with the standard interpretation of equality. Formulas $\varphi$ and $\psi$ subject to $\vdash(\varphi \Leftrightarrow \psi)$ are called logically equivalent.

Definition 2. The variables with free occurrences in a formula or term are called the parameters of the latter. The parameters of a set of formulas or terms are the variables contained in the union of the parameters of formulas and terms in the set. Formulas and terms having no parameters are called closed; closed formulas are also called sentences. If $x_{1}, \ldots, x_{n}$ is the complete list of parameters of a formula $\varphi$, then the closed formula $\left(\forall x_{1}\right) \ldots\left(\forall x_{n}\right) \varphi$ is called the universal closure of $\varphi$ and denoted by $(\forall \ldots) \varphi$. Given a set $\mathcal{F}$ of formulas, put $(\forall \ldots) \mathcal{F}:=\{(\forall \ldots) \varphi: \varphi \in \mathcal{F}\}$.

Definition 3. Expressions of the form $\ddot{x}$ or $\dddot{\tau}$ are used to denote arbitrary finite lists of variables or terms. The cardinality $n$ of a list $\ddot{\tau}=\tau_{1}, \ldots, \tau_{n}$ is denoted by $|\ddot{\tau}|$. The formulas

$$
\tau_{1}=\sigma_{1} \wedge \cdots \wedge \tau_{n}=\sigma_{n}, \quad \tau_{1} \in \sigma_{1} \wedge \cdots \wedge \tau_{n} \in \sigma_{n}, \quad \tau_{1} \in \sigma \wedge \cdots \wedge \tau_{n} \in \sigma
$$

are abbreviated as $\dddot{\tau}=\ddot{\sigma}, \dddot{\tau} \in \ddot{\sigma}$, and $\dddot{\tau} \in \sigma$.

Agreement 1. We assume that the set of all variables is computably organized into a sequence and call the corresponding order the alphabetical order. By saying " $\varphi$ is a formula with parameters $\dddot{x} "$ or " $\dddot{x}$ are the parameters of $\varphi$ " we always mean that $\ddot{x}$ is the complete list of parameters of $\varphi$ listed without duplicates in the alphabetical order. By the parameters of a finite set of formulas we mean the union of their parameters listed without duplicates in the alphabetical order. The same is true of the parameters of terms and of finite sets of terms.

Agreement 2. In what follows, the words "a new variable" or "new variables" stand for the alphabetically first variables that do not occur in the preceding formulas or terms under consideration. This agreement is necessary for making the constructions well-defined and keeping the procedures computable (see, for instance, Definition $7(\mathrm{~d})$ and Definition 21).

We make a conventional agreement that simplifies the syntax of term substitution. 
Agreement 3. When writing a formula $\varphi$ initially as $\varphi\left(x_{1}, \ldots, x_{n}\right)$, with $x_{1}, \ldots, x_{n}$ presupposed to be pairwise different variables, we do not assume that all the variables $x_{1}, \ldots, x_{n}$ participate in $\varphi$ as parameters. We also do not assume that all the parameters of $\varphi$ belong to the list $x_{1}, \ldots, x_{n}$. The initial notation $\varphi\left(x_{1}, \ldots, x_{n}\right)$ only means that every subsequent expression of the form $\varphi\left(\tau_{1}, \ldots, \tau_{n}\right)$ denotes the result $\left.\varphi\right|_{\tau_{1}, \ldots, \tau_{n}} ^{x_{1}, \ldots, x_{n}}$ of simultaneous substitution of the terms $\tau_{1}, \ldots, \tau_{n}$ in $\varphi$ for $x_{1}, \ldots, x_{n}$ (with possible name collisions eliminated by renaming the bound variables of $\varphi$ occurred in $\tau_{i}$ ). If $x_{1}, \ldots, x_{n}$ are not among the parameters of $\tau_{1}, \ldots, \tau_{n}$, then the formula $\varphi\left(\tau_{1}, \ldots, \tau_{n}\right)$ is logically equivalent to

$$
\left(\exists x_{1}\right) \ldots\left(\exists x_{n}\right)\left(x_{1}=\tau_{1} \wedge \cdots \wedge x_{n}=\tau_{n} \wedge \varphi\right) ;
$$

while in the general case we have

$$
\vdash \varphi\left(\tau_{1}, \ldots, \tau_{n}\right) \Leftrightarrow\left(\exists y_{1}\right) \ldots\left(\exists y_{n}\right)\left(y_{1}=\tau_{1} \wedge \cdots \wedge y_{n}=\tau_{n} \wedge \varphi\left(y_{1}, \ldots, y_{n}\right)\right),
$$

where $y_{1}, \ldots, y_{n}$ are new variables (see Agreement 2).

The analogous agreement is proposed about the notation of the form $\tau\left(x_{1}, \ldots, x_{n}\right)$ and its relation to the result $\tau\left(\tau_{1}, \ldots, \tau_{n}\right)$ of simultaneous substitution of the terms $\tau_{1}, \ldots, \tau_{n}$ in the term $\tau$ for $x_{1}, \ldots, x_{n}$.

Definition 4. In what follows, $\mathbb{N}=\{1,2, \ldots\}$ is the set of naturals; $\omega=\{0,1,2, \ldots\}$ is the least infinite ordinal. The class of all ordinals is denoted by Ord; and the class of all limit ordinals, by Lim Ord. Moreover, we use the notation

$$
\operatorname{Ord}^{\bullet}:=\operatorname{Ord} \cup\{\infty\}, \quad \operatorname{Lim} \text { Ord }:=\operatorname{Lim} \text { Ord } \cup\{\infty\},
$$

where $\infty \notin$ Ord and $\alpha<\infty$ for all $\alpha \in$ Ord. The symbol " $\subset$ " stands for the non-strict inclusion.

\subsection{Eliminable Extensions}

After examining several examples of definitions, we formalize the notion of definition as an eliminable extension of a theory; present a useful criterion for the eliminability of an extension; clarify the notions of correct and conditionally correct definition; list the key properties of an elimination of definable symbols; and justify iterative definitions and the union of independent definitions.

We start with a brief description of a possible formalism behind introduction of new notation and terminology, that is, extension of the language of a theory by means of definitions of new formulas and terms, such as $x \subset y, f: x \rightarrow y, \mathcal{P}(x), x \cup y, \varnothing, \mathbb{N}$.

The role of the formal language of set theory is conventionally played by the firstorder predicate language $F(\boldsymbol{\epsilon})$ of formulas of signature $\mathcal{\epsilon}=\{=, \in\}$. Initially, the language consists of the atomic formulas $x=y$ and $x \in y$ (with $x$ and $y$ arbitrary variables) and the formulas recursively constructed from simpler formulas by means of propositional and quantifier connectives.

Suppose that we would like to extend the language of set theory with the new formula $x \subset y$ and the two new terms $\mathcal{P}(x)$ and $\varnothing$. To this end, it suffices to consider the signature $\epsilon^{*}=\{=, \in, \subset, \mathcal{P}, \varnothing\}$ that enriches $\mathcal{\epsilon}$ with the binary predicate symbol $\subset$, unary function symbol $\mathcal{P}$, and constant $\varnothing$. As a result, the formal language $F\left(\epsilon^{*}\right)$ of the extended signature $\epsilon^{*}$ contains such new atomic formulas as $\varnothing \in x, \mathcal{P}(x) \subset \mathcal{P}(\mathcal{P}(\varnothing))$, and so forth, as well as various formulas recursively constructed from the new formulas, including, for instance, the formula $(\exists x)(\varnothing \in x \wedge \mathcal{P}(x) \subset \mathcal{P}(\mathcal{P}(\varnothing)))$ that literally belongs to the extended language $F\left(\epsilon^{*}\right)$ and does not contain any abbreviations or informal notation.

By enriching the signature $\epsilon$ to $\epsilon^{*}$ we extended the language with some new expressions but did not make them "sensible." The task can be performed by adding axioms that play the role of the corresponding definitions. Consider the formulas 


$$
\begin{aligned}
\operatorname{Sub}(x, y) & :=(\forall z)(z \in x \Rightarrow z \in y), \\
\operatorname{Pwr}(x, y) & :=(\forall z)(z \in y \Leftrightarrow \operatorname{Sub}(z, x)), \\
\operatorname{Emp}(x) & :=\neg(\exists z)(z \in x)
\end{aligned}
$$

and denote by $\mathrm{ZFC}^{*}$ the theory of signature $\epsilon^{*}$ obtained from ZFC by adding the following three special axioms:

$$
x \subset y \Leftrightarrow \operatorname{Sub}(x, y), \quad y=\mathcal{P}(x) \Leftrightarrow \operatorname{Pwr}(x, y), \quad x=\varnothing \Leftrightarrow \operatorname{Emp}(x) .
$$

Since the formulas $\operatorname{Sub}(x, y), \operatorname{Pwr}(x, y)$, and $\operatorname{Emp}(x)$ belong to the language of signature $\boldsymbol{\epsilon}$, every formula $\varphi$ of the extended language $F\left(\boldsymbol{\epsilon}^{*}\right)$ admits a "translation" into the initial language $F(\boldsymbol{\epsilon})$, an equivalent formula $\lceil\varphi\rceil$ of signature $\boldsymbol{\epsilon}$. The translation procedure can be organized recursively by passing through the logical connectives and transforming the atomic formulas of signature $\epsilon^{*}$ according to the above definitions:

$$
\begin{array}{r}
\lceil\varphi \wedge \psi\rceil=\lceil\varphi\rceil \wedge\lceil\psi\rceil, \quad\lceil\neg \varphi\rceil=\neg\lceil\varphi\rceil, \quad\lceil(\exists x) \varphi\rceil=(\exists x)\lceil\varphi\rceil, \\
\lceil x=y\rceil=x=y, \quad\lceil x \in y\rceil=x \in y, \\
\lceil\sigma \subset \tau\rceil=\lceil\operatorname{Sub}(\sigma, \tau)\rceil, \quad\lceil\tau=\mathcal{P}(\sigma)\rceil=\lceil\operatorname{Pwr}(\sigma, \tau)\rceil, \quad\lceil\tau=\varnothing\rceil=\lceil\operatorname{Emp}(\tau)\rceil, \\
\lceil\mathcal{P}(\sigma) \in \tau\rceil=(\exists y)(\lceil y=\mathcal{P}(\sigma)\rceil \wedge\lceil y \in \tau\rceil), \quad\lceil\varnothing \in \tau\rceil=(\exists y)(\lceil y=\varnothing\rceil \wedge\lceil y \in \tau\rceil), \\
\lceil\tau \in \mathcal{P}(\sigma)\rceil=(\exists y)(\lceil y=\mathcal{P}(\sigma)\rceil \wedge\lceil\tau \in y\rceil), \quad\lceil\tau \in \varnothing\rceil=(\exists y)(\lceil y=\varnothing\rceil \wedge\lceil\tau \in y\rceil),
\end{array}
$$

where $x$ and $y$ are variables; $\sigma$ and $\tau$ are terms of signature $\epsilon^{*} ; \varphi$ and $\psi$ are formulas of signature $\epsilon^{*}$.

It is important to note that the formulas $(\forall x)(\exists ! y) \operatorname{Pwr}(x, y)$ and $(\exists ! x) \operatorname{Emp}(x)$ are provable in ZFC, which guarantees the correctness of the definitions introduced: formal reasoning within the extended theory $\mathrm{ZFC}^{*}$ belongs to legal deduction means, that is, the use of definitions does not make it possible to prove anything unprovable in the pure ZFC.

Definition 5. Guided by the above example, we may conclude that a definition, or an introduction of new notation, is a conservative extension of the theory which admits elimination, "restatement" of the assertions of the extended language in terms of the initial language.

Consider a theory $\mathcal{T}$ of signature $\Sigma$. An eliminable extension of $\mathcal{T}$, or an extension of $\mathcal{T}$ by means of definitions, is an extension of $\mathcal{T}$ to a theory $\mathcal{T}^{*}$ of a richer signature $\Sigma^{*}$ subject to the following conditions:

(a) $\mathcal{T}^{*}$ is a conservative extension of $\mathcal{T}$, that is, $\mathcal{T}^{*} \vdash \varphi$ implies $\mathcal{T} \vdash \varphi$ for each $\varphi \in F(\Sigma)$;

(b) for each $\varphi \in F\left(\Sigma^{*}\right)$ there exists $\psi \in F(\Sigma)$ such that $\mathcal{T}^{*} \vdash(\varphi \Leftrightarrow \psi)$.

(Analogs of the notion of eliminable extension can be found in the literature under the name of definitional or inessential extension.) The special axioms of $\mathcal{T}^{*}$ that do not belong to $\mathcal{T}$ are called the definitions or the defining axioms; the formula $\psi$ in (b) can be called a translation of $\varphi$ into the language of $\Sigma$ or a restatement of $\varphi$ in terms of $\Sigma$.

Observe that the set $\Sigma^{*} \backslash \Sigma$ of new symbols can be infinite (see, e.g., Definitions 6 and 11). Nevertheless, due to the requirement that the signatures and the sets of axioms are decidable (see Definition 1), each eliminable extension $\mathcal{T} \subset \mathcal{T}^{*}$ admits an elimination in the form of a computable function

$$
\lceil\cdot\rceil: \varphi \in F\left(\Sigma^{*}\right) \mapsto\lceil\varphi\rceil \in F(\Sigma)
$$

mapping each formula $\varphi$ of signature $\Sigma^{*}$ to a formula $\lceil\varphi\rceil$ of signature $\Sigma$ so that

$$
\mathcal{T}^{*} \vdash(\varphi \Leftrightarrow\lceil\varphi\rceil) .
$$

We present a criterion for the eliminability of an extension that is easily verifiable for the majority of definitions occurred in mathematical practice. 
Theorem 1. Let $\Sigma^{*}$ be a signature that enriches a signature $\Sigma$ by a set $P$ of new predicate symbols and by a set $F$ of new function symbols, and let $\mathcal{T}^{*}$ be a theory of signature $\Sigma^{*}$ that extends a theory $\mathcal{T}$ of signature $\Sigma$. The theory $\mathcal{T}^{*}$ is an eliminable extension of $\mathcal{T}$ if and only if there is a function mapping the symbols $s \in P \cup F$ to formulas $s_{\Sigma}$ of signature $\Sigma$ so that

$$
\begin{aligned}
& \mathcal{T}^{*} \vdash\left(p(\dddot{x}) \Leftrightarrow p_{\Sigma}(\dddot{x})\right) \quad(p \in P), \\
& \mathcal{T}^{*} \vdash\left(y=f(\ddot{x}) \Leftrightarrow f_{\Sigma}(\ddot{x}, y)\right) \quad(f \in F), \\
& \mathcal{T} \vdash(\exists ! y) f_{\Sigma}(\dddot{x}, y) \quad(f \in F), \\
& \mathcal{T}^{+} \vdash \mathcal{T}^{*} \backslash \mathcal{T} \text {, }
\end{aligned}
$$

where $\mathcal{T}^{+}$is the theory obtained from $\mathcal{T}$ by adding the special axioms

$$
\begin{aligned}
p(\ddot{x}) & \Leftrightarrow p_{\Sigma}(\dddot{x}) & & (p \in P), \\
y=f(\dddot{x}) & \Leftrightarrow f_{\Sigma}(\ddot{x}, y) & & (f \in F) .
\end{aligned}
$$

Proof. Sufficiency: The conservativity of the extension $\mathcal{T} \subset \mathcal{T}^{*}$ can be easily verified with the help of the Soundness and Completeness theorems. An elimination for $\mathcal{T}^{*}$ can be defined by starting with the elimination available for the atomic formulas and then recursively extending it to all formulas by preserving the logical connectives.

Necessity: Given $p \in P$ and $f \in F$, put $p_{\Sigma}(\ddot{x})=\lceil p(\ddot{x})\rceil^{*}$ and $f_{\Sigma}(\ddot{x}, y)=\lceil y=f(\ddot{x})\rceil^{*}$, where $\lceil\cdot]^{*}$ is an elimination of the extension $\mathcal{T} \subset \mathcal{T}^{*}$. We only need to verify the condition $\mathcal{T}^{+} \vdash \mathcal{T}^{*} \backslash \mathcal{T}$. Consider an arbitrary $\varphi \in \mathcal{T}^{*}$. The above-proven "sufficiency" implies that the extension $\mathcal{T} \subset \mathcal{T}^{+}$admits an elimination $\lceil\cdot\rceil^{+}$. Since $\mathcal{T}^{*} \vdash\left(\varphi \Leftrightarrow\lceil\varphi\rceil^{*}\right)$, $\mathcal{T}^{+} \vdash\left(\varphi \Leftrightarrow\lceil\varphi\rceil^{+}\right)$, and $\mathcal{T}^{*} \vdash \mathcal{T}^{+}$; we have $\mathcal{T}^{*} \vdash\left(\lceil\varphi\rceil^{+} \Leftrightarrow\lceil\varphi\rceil^{*}\right)$ and so $\mathcal{T} \vdash\left(\lceil\varphi\rceil^{+} \Leftrightarrow\lceil\varphi\rceil^{*}\right)$. Taking account of $\mathcal{T}^{*} \vdash \varphi$, we successively deduce $\mathcal{T}^{*} \vdash\lceil\varphi\rceil^{*}, \mathcal{T} \vdash\lceil\varphi\rceil^{*}, \mathcal{T} \vdash\lceil\varphi\rceil^{+}$, $\mathcal{T}^{+} \vdash\lceil\varphi\rceil^{+}, \mathcal{T}^{+} \vdash \varphi$

Agreement 4. The condition $\mathcal{T} \vdash(\exists ! y) f_{\Sigma}(\ddot{x}, y)$ in Theorem 1 is conventionally called the correctness of the definition $y=f(\ddot{x}) \Leftrightarrow f_{\Sigma}(\ddot{x}, y)$. (If the correctness is violated, the extension $\mathcal{T}^{*}$ of a consistent theory $\mathcal{T}$ fails to be conservative.) Nevertheless, mathematical practice is replete with examples of terms $f(\ddot{x})$ being correctly defined only under certain conditions $\delta(\ddot{x})$ on the parameters $\dddot{x}=x_{1}, \ldots, x_{n}$ :

$$
\begin{aligned}
\mathcal{T} \vdash(\delta(\dddot{x}) & \left.\Rightarrow(\exists ! y) f_{\Sigma}(\ddot{x}, y)\right), \\
\mathcal{T}^{*} \vdash(\delta(\dddot{x}) & \left.\Rightarrow\left(y=f(\ddot{x}) \Leftrightarrow f_{\Sigma}(\dddot{x}, y)\right)\right) .
\end{aligned}
$$

Such a conditionally correct definition can always be made correct by letting $f(\ddot{x})=x_{1}$ in the case of $\neg \delta(\ddot{x}): \quad \quad \mathcal{T}^{*} \vdash\left(y=f(\dddot{x}) \Leftrightarrow\left(\delta(\ddot{x}) \wedge f_{\Sigma}(\dddot{x}, y)\right) \vee\left(\neg \delta(\ddot{x}) \wedge y=x_{1}\right)\right)$.

The above modification is implicitly assumed to be applied to each conditionally correct definition.

Agreement 5. As is easily seen, every elimination $\lceil\cdot\rceil$ of an eliminable extension $\mathcal{T} \subset \mathcal{T}^{*}$ translates formulas of the initial language to equivalent formulas: $\mathcal{T} \vdash(\varphi \Leftrightarrow\lceil\varphi\rceil)$ for all $\varphi \in F(\Sigma)$. The elimination is also invariant with respect to the logical connectives: $\mathcal{T} \vdash(\lceil\varphi \wedge \psi\rceil \Leftrightarrow\lceil\varphi\rceil \wedge\lceil\psi\rceil)$ and so forth. Moreover, the translation procedure can always be reorganized so that

(a) the initial formulas are unchanged: $\lceil\varphi\rceil=\varphi$ for all $\varphi \in F(\Sigma)$;

(b) the logical connectives are preserved:

$\lceil\varphi \wedge \psi\rceil=\lceil\varphi\rceil \wedge\lceil\psi\rceil,\lceil\neg \varphi\rceil=\neg\lceil\varphi\rceil,\lceil(\exists x) \varphi\rceil=(\exists x)\lceil\varphi\rceil$, etc.;

(c) each formula $\varphi$ is translated to a formula $\lceil\varphi\rceil$ with the same parameters.

In what follows, we assume that every elimination under consideration possesses the abovelisted properties (a)-(c).

Due to property (b), the translation of a formula does not depend on the context in which the formula is contained in superformulas. We thus may regard any formula $\varphi$ of the extended signature $\Sigma^{*}$ as a synonym (denotation, shorthand) for its translation $\lceil\varphi\rceil$ into the initial language of $\Sigma$ and handle the new formulas so as if they belong to the formal language of the basic theory $\mathcal{T}$ under consideration. 
Remark 1. If $\mathcal{T}_{1}$ is an eliminable extension of $\mathcal{T}$, and $\mathcal{T}_{2}$ is an eliminable extension of $\mathcal{T}_{1}$, then $\mathcal{T}_{2}$ is an eliminable extension of $\mathcal{T}$. This trivial observation justifies iterative definitions of new symbols by means of those previously defined.

Remark 2. Let $\mathcal{T}$ be a theory of signature $\Sigma$, and let $\mathcal{T}_{1}$ and $\mathcal{T}_{2}$ be eliminable extensions of $\mathcal{T}$ of signatures $\Sigma_{1}$ and $\Sigma_{2}$, with $\Sigma_{1} \cap \Sigma_{2}=\Sigma$. Then the theory $\mathcal{T}_{1} \cup \mathcal{T}_{2}$ of signature $\Sigma_{1} \cup \Sigma_{2}$ is an eliminable extension of $\mathcal{T}$ (cp. [7] (Theorem 20.6)). This justifies correctness of the union of independent systems of definitions.

\subsection{Classes in Set Theory}

After introducing the syntax of subclasses of sets as an eliminable extension, we will formalize the extension of the language of set theory by arbitrary definable, or internal, classes with the aid of so-called syntactic sugar.

Definition 6. In order to demonstrate an eliminable extension of ZFC with infinite set of new signature symbols, we will formalize the enrichment of the language of set theory by terms of the form $\{x \in y: \varphi\}$.

Let $x$ and $y$ be different variables and let $\varphi(x, y, \ddot{z})$ be a formula, where $z=z_{1}, \ldots, z_{n}$ is the alphabetically ordered list of all parameters of $\varphi$ other than $x$ and $y$. For each triple $x, y, \varphi$ described above, enrich the signature of set theory by the function symbol $\langle x \in y: \varphi\rangle$ of arity $n+1$, introduce the abbreviation

and add the defining axiom

$$
\{x \in y: \varphi(x, y, \ddot{z})\}:=\langle x \in y: \varphi(x, y, \ddot{z})\rangle(y, \ddot{z}),
$$

$$
x \in\{x \in y: \varphi(x, y, \ddot{z})\} \Leftrightarrow x \in y \wedge \varphi(x, y, \ddot{z}) .
$$

Due to the obvious provability of the equality

$$
\langle x \in y: \varphi(x, y, \ddot{z})\rangle(v, \ddot{w})=\{u \in v: \varphi(u, v, \ddot{w})\}
$$

(with $v$ and $\ddot{w}=w_{1}, \ldots, w_{n}$ arbitrary variables, and $u$ a variable different from $\left.v, \ddot{w}\right)$, we can avoid using the general expressions of the form $\langle x \in y: \varphi\rangle(\ldots)$ and confine ourselves to the use of the terms $\{x \in y: \varphi\}$.

Remark 3. Since the new symbols $\langle x \in y: \varphi\rangle$ were introduced for the language whose signature had not contained those symbols, the language has not been enriched by expressions of the form

$$
\{x \in\{y \in \sigma: \psi\}: \varphi(\{z \in \tau: \chi\}, \ldots)\} .
$$

This restriction can be removed, for instance, by the union of the sequence of extensions, each of which enlarges the admissible nesting depth of the new constructions in each other. The corresponding procedure can be called the grammatical closure (cp. (1)).

The formalism of eliminable extensions described in Definition 5 does not allow us to enrich the language of ZFC by the terms $\{x: \varphi\}$ of arbitrary definable classes and, in particular, by the terms $\mathbb{V}$ and $\mathbb{V}^{(B)}$. (No consistent extension of ZFC can provide the theorem $(\forall x)(x \in \mathbb{V})$ for a term $\mathbb{V}$, since the formula $(\exists y)(y=\mathbb{V})$ is deducible in the predicate calculus.) Similarly to the case of eliminable extension, extension of the language by the syntax of definable classes assumes enrichment of the signature by new symbols; but the theory per se is not extended, and the role of elimination is played by the so-called syntactic sugar, an explicit translation procedure of the formulas of the extended language into the language of the initial signature.

In this subsection, when writing a formula as $\varphi(x, \ddot{y})$, agree to suppose that $\ddot{y}$ is the complete list of parameters of $\varphi$ other than $x$, while $x$ need not participate in $\varphi$. 
Definition 7. Let $\Sigma$ be an arbitrary signature. For each pair $x, \varphi$, with $x$ a variable and $\varphi(x, \dddot{y})$ a formula of signature $\Sigma$, consider the function symbol $\langle x: \varphi\rangle$ of arity $|\ddot{y}|$ and introduce the notation $\{x: \varphi\}$ for the term $\langle x: \varphi\rangle(\dddot{y})$ :

$$
\{x: \varphi(x, \dddot{y})\}:=\langle x: \varphi(x, \dddot{y})\rangle(\dddot{y}) .
$$

The symbols $\langle x: \varphi\rangle$ are called class symbols, and the terms $\{x: \varphi\}$ are classes or, more exactly, definable classes or internal classes. Denote by $\mathrm{cl} \Sigma$ the signature obtained from $\Sigma$ by adding the class symbols $\langle x: \varphi\rangle$, with $\varphi \in F(\Sigma)$. Let $\mathrm{cl}^{\omega} \in$ be the smallest enrichment of the standard set-theoretic signature $\epsilon=\{=, \in\}$ that is closed under the formation of classes:

$$
\begin{aligned}
\mathrm{cl}^{0} \epsilon & =\epsilon ; \\
\mathrm{cl}^{n+1} \epsilon & =\mathrm{cl}\left(\mathrm{cl}^{n} \epsilon\right), \quad n \in \omega ; \\
\mathrm{cl}^{\omega} \epsilon & =\bigcup_{n \in \omega} \mathrm{cl}^{n} \epsilon .
\end{aligned}
$$

There exists a unique mapping $\lceil\cdot\rceil: F\left(\mathrm{cl}^{\omega} \boldsymbol{\epsilon}\right) \rightarrow F(\boldsymbol{\epsilon})$ subject to the following conditions:

(a) $\lceil\cdot\rceil$ is identical on $F(\boldsymbol{\epsilon})$, that is, $\lceil\varphi\rceil=\varphi$ for the formulas $\varphi$ of signature $\epsilon$;

(b) $\lceil\cdot\rceil$ preserves the logical connectives, that is,

$\lceil\varphi \wedge \psi\rceil=\lceil\varphi\rceil \wedge\lceil\psi\rceil,\lceil\neg \varphi\rceil=\neg\lceil\varphi\rceil,\lceil(\exists x) \varphi\rceil=(\exists x)\lceil\varphi\rceil$, etc.;

(c) for all variables $x$ and $y$, all formulas $\varphi\left(x, y_{1}, \ldots, y_{n}\right)$ of signature $\mathrm{cl}^{\omega} \epsilon$, and all terms $\tau_{1}, \ldots, \tau_{n}$ of signature $\mathrm{cl}^{\omega} \epsilon$,

$$
\left\lceil y \in\langle x: \varphi\rangle\left(\tau_{1}, \ldots, \tau_{n}\right)\right\rceil=\left\lceil\left.\varphi\left(x, \tau_{1}, \ldots, \tau_{n}\right)\right|_{y} ^{x}\right\rceil ;
$$

(d) for each variable $x$ and all terms $\tau, \sigma$ of signature $\mathrm{cl}^{\omega} \in$ that are not variables,

$$
\begin{aligned}
\lceil x=\tau\rceil=\lceil\tau=x\rceil & =(\forall u)(\lceil u \in \tau\rceil \Leftrightarrow u \in x), \\
\lceil\tau \in x\rceil & =(\exists u)(\lceil u=\tau\rceil \wedge u \in x), \\
\lceil\tau=\sigma\rceil & =(\forall u)(\lceil u \in \tau\rceil \Leftrightarrow\lceil u \in \sigma\rceil), \\
\lceil\tau \in \sigma\rceil & =(\exists u)(\lceil u=\tau\rceil \wedge\lceil u \in \sigma\rceil),
\end{aligned}
$$

where $u$ is a new variable (see Agreement 2).

The mapping $\lceil\cdot\rceil$ is called the elimination of classes.

Say that terms $\sigma$ and $\tau$ of signature $\mathrm{cl}^{\omega} \epsilon$ are syntactically equivalent and write $\sigma \equiv \tau$, whenever $\sigma$ and $\tau$ are interchangeable without affecting the result of elimination, that is, $\left\lceil\left.\varphi\right|_{\sigma} ^{x}\right\rceil=$ $\left\lceil\left.\varphi\right|_{\tau} ^{x}\right\rceil$ for every formula $\varphi$ of signature $\mathrm{cl}^{\omega} \epsilon$ and every variable $x$. From condition (d) it is clear that the equivalence $\sigma \equiv \tau$ amounts to the equality $\lceil u \in \tau\rceil=\lceil u \in \sigma\rceil$, where $u$ is not a parameter of $\tau$ or $\sigma$. Moreover, if $\varphi\left(x, y_{1}, \ldots, y_{n}\right)$ is a formula of signature $\mathrm{cl}^{\omega} \epsilon, \tau_{1}, \ldots, \tau_{n}$ are terms of signature $\mathrm{cl}^{\omega} \epsilon$, and $\psi\left(x, z_{1}, \ldots, z_{m}\right):=\varphi\left(x, \tau_{1}, \ldots, \tau_{n}\right)$; then, according to (c), we have

$$
\begin{aligned}
\lceil u \in\langle x: \varphi(x, \dddot{y})\rangle(\dddot{\tau})\rceil & =\left\lceil\left.\varphi(x, \ddot{\tau})\right|_{u} ^{x}\right\rceil=\left\lceil\left.\psi(x, \dddot{z})\right|_{u} ^{x}\right\rceil=\lceil u \in\langle x: \psi(x, \ddot{z})\rangle(\dddot{z})\rceil \\
& =\lceil u \in\{x: \psi(x, \ddot{z})\}\rceil=\lceil u \in\{x: \varphi(x, \dddot{\tau})\}\rceil
\end{aligned}
$$

and, consequently,

$$
\langle x: \varphi(x, \dddot{y})\rangle(\dddot{\tau}) \equiv\{x: \varphi(x, \dddot{\tau})\} .
$$

Therefore, every term of signature $\mathrm{cl}^{\omega} \epsilon$ is syntactically equivalent to a suitable class, and so we may refuse to employ expressions of the form $\langle x: \varphi\rangle(\ldots)$ and confine ourselves to the use of classes $\{x: \varphi\}$ without decreasing the expressive power of the language (cp. Definition 6).

The parameters of a class $\{x: \varphi\}$ are the parameters of the class as a term of signature $\mathrm{cl}^{\omega} \epsilon$, which evidently coincide with the parameters of $\varphi$ other than $x$. For instance, if the formula $v(x, B)$ of signature $\epsilon$ expresses the containment of a set $x$ to the classical B-valued universe, then the term

$$
\mathbb{V}^{(B)}:=\{x: v(x, B)\}=\langle x: v(x, B)\rangle(B)
$$


of signature $\mathrm{cl}^{\omega} \boldsymbol{\epsilon}$ is a class with parameter $B$.

Remark 4. Due to Definition 7, the language of each theory of set-theoretic signature $\epsilon$ can be extended by the use of classes. Moreover, the extension is purely syntactic and has no relation to the theory. The enrichment of the signature $\epsilon \subset \mathrm{cl}^{\omega} \epsilon$ is not accompanied by any extension of the axiomatics. In particular, classes do not become terms of the theory under consideration, and the logical axioms remain corresponding to the predicate calculus of the initial signature $\epsilon$. (For instance, if $x$ and $y$ are variables, $C$ is a class, and the theory does not contain the axiom of extensionality, then the formula $(x=C \wedge y=C) \Rightarrow x=y$ needs not to be a theorem.) In this respect, formulas of signature $\mathrm{cl}^{\omega} \epsilon$ are not full-fledged participants of the theory and, due to the syntactic sugar, are regarded as synonyms (denotations, shorthands) for the results of elimination of classes applied to them.

Conditions (a)-(d) of Definition 7 determine the elimination of classes only for the formulas of signature $\mathrm{cl}^{\omega} \epsilon$ and are not applicable to any extension by means of definitions. If classes are used in the language of an eliminable extension, then, for translating a formula into the initial language of signature $\boldsymbol{\epsilon}$, we should, first, apply an elimination of the extension (in order to obtain a formula of signature $\left.\mathrm{cl}^{\omega} \boldsymbol{\epsilon}\right)$ and, next, eliminate the classes.

Agreement 6. Terms are conventionally regarded as particular cases of classes, since every eliminable extension of ZFC proves the equality $\tau=\{x: x \in \tau\}$ for each term $\tau$ (whose parameters do not include $x$ ). Therefore, when calling any symbol $X$ a class, we do not exclude the case in which $X$ is a variable or a term definable by an eliminable extension of ZFC.

The above agreement does not mean replacement of set theory by any theory of classes and does not extend the language of formulas by quantifiers over classes. Even if a symbol $X$ is chosen to mean a class $\{x: \varphi(x)\}$, the expression $(\exists Y \subset X) \psi(Y)$, which is a shorthand for the formula $(\exists Y)((\forall x)(x \in Y \Rightarrow \varphi(x)) \wedge \psi(Y))$, is interpreted as existence of a set (not a class) $Y \subset X$ possessing the property $\psi(Y)$.

The phrases "for all classes" and "there exists a class" are meta-quantifiers. The corresponding statements usually produce infinite assertions (see Section 2.4 below) and are formalized on the meta-theoretical level. For instance, if $\varphi(x)$ and $\psi(x, y)$ are formulas, and the statement

"for every class $X, \varphi(X)$ if and only if $\psi(X, Y)$ for some class $Y$ "

is asserted to be a theorem of ZFC, then the conjunction of the following two meta-assertions is actually meant:

(a) for an arbitrary class $X$, there is a class $Y$ such that $\mathrm{ZFC} \vdash(\varphi(X) \Rightarrow \psi(X, Y))$;

(b) for arbitrary classes $X$ and $Y$, ZFC $\vdash(\psi(X, Y) \Rightarrow \varphi(X))$.

Definition 8. If $X$ is a class, then the phrase " $X$ is a set" means the formula $(\exists y)(y=X)$ or, more exactly, $(\exists y)\lceil y=X\rceil$. (Here $y$ is not a parameter of $X$.) The negation $\neg(\exists y)\lceil y=X\rceil$ formalizes the phrase " $\mathrm{X}$ is a proper class."

\subsection{Infinite Assertions}

Infinite assertions are specific for the subject under consideration. Those are infinite sets of formulas. For instance, the assertion " $\psi(X)$ is valid for all classes $X$ " is constituted by all the formulas $\psi(\{x: \varphi\})$, with $x$ a variable and $\varphi$ an arbitrary formula. The examples of infinite assertions are also " $X$ is a model of ZFC" (see Proposition 8) and " $X$ meets the maximum principle" (see Definition 43).

Mathematical texts often use "declarations of hypotheses." Such a declaration means that, within a fragment of reasoning, certain conditions are assumed to be valid or some variables are fixed and play the role of objects with certain properties. As an example serves the phrase "in what follows, $B$ is a complete Boolean algebra" that the next subsection 
of this article starts with. Actually, the phrase fixes the letter $B$ and adds a temporary axiom $\gamma(B)$ that formalizes the assertion " $B$ is a complete Boolean algebra."

In most cases, the effect of declaring a hypothesis is quite clear at the informal level, but the use of infinite sets of formulas as hypotheses or conclusions requires accuracy.

Logical connectives with infinite assertions make sense due to the apparatus of formal deduction. For instance, if at least one of the assertions $\Gamma$ or $\Delta$ is infinite, then the implication $\Gamma \Rightarrow \Delta$ itself has no sense; while the phrase " $\Gamma$ implies $\Delta$ within the theory $\mathcal{T}$ " can be formalized as $\mathcal{T}, \Gamma \vdash \Delta$, that is the deducibility of $\Delta$ from the hypotheses $\Gamma$ within $\mathcal{T}$.

Definition 9. Let $\mathcal{T}$ be a theory of signature $\Sigma$ and let $\Gamma$ and $\Delta$ be arbitrary sets of formulas of signature $\Sigma$. (The sets $\Gamma$ and $\Delta$ can be infinite and may have parameters.) Assume that the formulas in $\Delta$ do not contain quantifiers over any parameters of $\Gamma$. The deducibility of the conclusion $\Delta$ from the hypothesis $\Gamma$ within $\mathcal{T}$ is written as $\mathcal{T}, \Gamma \vdash \Delta$ and defined by means of the notion of formal deduction in predicate calculus: For every formula $\delta \in \Delta$ there is a finite sequence of formulas $\varphi_{1}, \ldots, \varphi_{n}$ such that $\varphi_{n}=\delta$ and each formula $\varphi_{i}$ either belongs to $\mathcal{T} \cup \Gamma$ or is obtained from the previous formulas $\varphi_{1}, \ldots, \varphi_{i-1}$ by one of the classical deduction rules save the rules with quantifiers over the parameters of $\Gamma$. Informally, the latter means that the parameters of the hypothesis are fixed and play the role of constants (cp. Proposition 3).

The propositions presented in this subsection are well known and can be easily verified by using the Soundness, Deduction, and Completeness theorems.

Proposition 1. Let $\mathcal{T}$ be a theory of signature $\Sigma$ and let $\Gamma$ and $\Delta$ be arbitrary sets of formulas of signature $\Sigma$ such that the parameters of $\Gamma$ are not quantified in $\Delta$. Then the following are equivalent:

(a) $\mathcal{T}, \Gamma \vdash \Delta$;

(b) $\mathcal{T}, \Gamma \vdash \delta$ for all $\delta \in \Delta$;

(c) for every $\delta \in \Delta$ there is a finite subset $\left\{\gamma_{1}, \ldots, \gamma_{n}\right\} \subset \Gamma$ such that $\mathcal{T} \vdash\left(\gamma_{1} \wedge \cdots \wedge \gamma_{n} \Rightarrow \delta\right)$ or, which is the same, $\mathcal{T} \vdash(\forall \ldots)\left(\gamma_{1} \wedge \cdots \wedge \gamma_{n} \Rightarrow \delta\right)$;

(d) for every model $X$ of $\mathcal{T}$ and every valuation $v: V \rightarrow X$ of the parameters $V$ of $\Gamma \cup \Delta$, the validity $X \vDash \gamma[v]$ of all $\gamma \in \Gamma$ implies the validity $X \vDash \delta[v]$ of all $\delta \in \Delta$.

Proposition 2. Let $\mathcal{T}$ be a theory of signature $\Sigma$ and let $\Gamma$ be an arbitrary set of formulas of signature $\Sigma$. Suppose that

$$
\mathcal{T} \vdash(\exists \dddot{z})\left(\gamma_{1} \wedge \cdots \wedge \gamma_{n}\right)
$$

for every finite subset $\left\{\gamma_{1}, \ldots, \gamma_{n}\right\} \subset \Gamma$ with parameters $\ddot{v}$. Then $\Gamma$ conservatively complements $\mathcal{T}$ in the following sense:

$$
\mathcal{T}, \Gamma \vdash \varphi \Leftrightarrow \mathcal{T} \vdash \varphi
$$

whenever $\varphi$ is a formula of signature $\Sigma$ and the parameters of $\Gamma$ do not occur in $\varphi$.

Another approach to a formalization of a decidable set of hypotheses $\Gamma$ consists in replacing the parameters of $\Gamma$ with new constants and extending the theory $\mathcal{T}$ with $\Gamma$ regarded as an additional set of axioms. After discarding the hypotheses in this way, we can use the standard deduction in the extended theory.

Proposition 3. Let $\mathcal{T}$ be a theory of signature $\Sigma$, let $\Gamma$ be a decidable set of formulas of signature $\Sigma$, and let $V=\left\{v_{1}, v_{2}, \ldots\right\}$ be the (finite or infinite) set of all parameters of $\Gamma$. Consider the signature $\Sigma \cup C$ obtained from $\Sigma$ by adding the set $C=\left\{c_{1}, c_{2}, \ldots\right\}$ of new constants. Given a formula $\varphi$ of signature $\Sigma$, denote by $\varphi(C)$ the result of substituting the constants $c_{i}$ for the free variables $v_{i}$ in $\varphi$. Let $\mathcal{T} \cup \Gamma(C)$ be the theory of signature $\Sigma \cup C$ obtained from $\mathcal{T}$ by adding the set of axioms $\Gamma(C)=\{\gamma(C): \gamma \in \Gamma\}$.

(a) If $\delta$ is a formula of signature $\Sigma$ without quantifiers over $V$, then the deducibility $\mathcal{T}, \Gamma \vdash \delta$ is equivalent to $\mathcal{T} \cup \Gamma(C) \vdash \delta(C)$.

(b) If $\mathcal{T} \vdash(\exists \ddot{v})\left(\gamma_{1} \wedge \cdots \wedge \gamma_{n}\right)$ for every finite subset $\left\{\gamma_{1}, \ldots, \gamma_{n}\right\} \subset \Gamma$ with parameters $\ddot{v}$, then $\mathcal{T} \cup \Gamma(C)$ is a conservative extension of $\mathcal{T}$. 
Declaration of a finite set of hypotheses $\left\{\gamma_{1}, \ldots, \gamma_{n}\right\}$ obviously reduces to the case of a single hypothesis $\gamma:=\gamma_{1} \wedge \cdots \wedge \gamma_{n}$ and admits a simpler formalization based on the following fact.

Proposition 4. Let $\mathcal{T}$ be a theory of signature $\Sigma$ and let $\gamma(\ddot{z})$ be a formula of signature $\Sigma$ with parameters $\ddot{v}=v_{1}, \ldots, v_{m}$. Consider the signature $\Sigma+\ddot{c}$ obtained from $\Sigma$ by adding the constants $\dddot{c}=c_{1}, \ldots, c_{m}$ and let $\mathcal{T}+\gamma(\ddot{c})$ be the theory of signature $\Sigma+\dddot{c}$ obtained from $\mathcal{T}$ by adding the axiom $\gamma(\ddot{c})$.

(a) For every formula $\delta(\ddot{v})$ of signature $\Sigma$ without quantifiers over $\ddot{v}$,

$$
\mathcal{T}+\gamma(\ddot{c}) \vdash \delta(\ddot{c}) \Leftrightarrow \mathcal{T}, \gamma \vdash \delta \Leftrightarrow \mathcal{T} \vdash(\gamma \Rightarrow \delta) \Leftrightarrow \mathcal{T} \vdash(\forall \ddot{v})(\gamma(\ddot{v}) \Rightarrow \delta(\ddot{v})) .
$$

(b) If $\mathcal{T} \vdash(\exists \ddot{v}) \gamma(\ddot{v})$ then $\mathcal{T}+\gamma(\ddot{c})$ is a conservative extension of $\mathcal{T}$.

For instance, under the hypothesis " $B$ is a complete Boolean algebra," the expression

$$
\mathrm{ZFC} \vdash\left(\mathbb{V}^{(B)} \vDash \mathrm{ZFC}\right),
$$

which symbolizes the provability in ZFC of the infinite assertion " $\mathbb{V}^{(B)}$ is a model of ZFC, is formally equivalent to the deducibility

$$
\text { ZFC, } B \text { is a complete Boolean algebra } \vdash\left(\mathbb{V}^{(B)} \vDash \mathrm{ZFC}\right) \text {; }
$$

the latter in turn means that, for every sentence $\varphi$ that is a theorem of ZFC, the following equivalent conditions hold:

(a) ZFC, $B$ is a complete Boolean algebra $\vdash\left(\mathbb{V}^{(B)} \vDash \varphi\right)$;

(b) ZFC $\vdash(\forall B)\left(B\right.$ is a complete Boolean algebra $\left.\Rightarrow \mathbb{V}^{(B)} \vDash \varphi\right)$;

(c) $\mathrm{ZFC}_{B} \vdash\left(\mathbb{V}^{(B)} \vDash \varphi\right)$,

where $\mathrm{ZFC}_{B}$ is the conservative extension of $\mathrm{ZFC}$ obtained by adding the constant $B$ and the axiom " $B$ is a complete Boolean algebra."

\subsection{Boolean-Valued Algebraic Systems}

In this subsection, we formalize the notion of Boolean-valued algebraic system, indicate the main syntactic properties of the Boolean truth valuation, and recall the basic notions related to Boolean-valued systems: extensional function, Boolean-valued class, model of a theory, separated system, subsystem, isomorphism.

Throughout the rest of the article we argue within ZFC. In particular, all the lemmas, propositions, and theorems are assumed to be stated and proven in ZFC. When introducing any definitions, declaring any hypotheses with parameters, or using internal classes, we implicitly enrich the signature and conservatively extend the axiomatics of the theory, but conventionally preserve the name of ZFC for the extended theory and identify the formulas $\varphi$ of the extended language with the formulas $\lceil\varphi\rceil$ of the initial language obtained by the corresponding eliminations.

In what follows, $B$ is a complete Boolean algebra.

Definition 10. By saying that $X$ is a B-presystem of signature $\Sigma$, we mean that

(a) $X$ is a class;

(b) $\Sigma$ is a predicative signature, that is, a signature that consists of only predicate symbols;

(c) a computable function $p \mapsto[p]_{X}$ is defined that maps symbols $p \in \Sigma$ to classes $[p]_{X}$;

(d) $X \neq \varnothing$ and $[p]_{X}: X^{n} \rightarrow B$ for each $n$-ary predicate symbol $p \in \Sigma$.

The class functions $[p]_{X}$ are called the B-valued interpretations of the symbols $p \in \Sigma$. 
Assertion (d), that is infinite in case $\Sigma$ is infinite, is assumed to be appended to ZFC as a hypothesis whose parameters are $B$ and the optional parameters of $X$ and $[p]_{X}$; see Section 2.4.

Definition 11. Given a B-presystem $X$ of signature $\Sigma$, define the truth values $[\varphi]_{X}$ of formulas $\varphi$ along the lines of Definition 6. Namely, for each formula $\varphi$ of signature $\Sigma$ with parameters $\ddot{x}$, introduce the function symbol $\langle\varphi\rangle_{X}$ of arity $|\ddot{x}|$, agree to write the term $\langle\varphi(\ddot{x})\rangle_{X}(\ddot{x})$ as $[\varphi]_{X}$, and extend the theory by the definitions

$$
\begin{gathered}
X=[p]_{X}(\ddot{x}), \\
{[\varphi \vee \psi]_{X}=[\varphi]_{X} \vee_{B}[\psi]_{X}, \quad[\varphi \wedge \psi]_{X}=[\varphi]_{X} \wedge_{B}[\psi]_{X},} \\
{[\neg \varphi]_{X}=\neg_{B}[\varphi]_{X}, \quad[\varphi \Rightarrow \psi]_{X}=\neg_{B}[\varphi]_{X} \vee_{B}[\psi]_{X}} \\
{[(\exists x) \varphi]_{X}=\vee_{B}\left\{[\varphi]_{X}: x \in X\right\}, \quad[(\forall x) \varphi]_{X}=\wedge_{B}\left\{[\varphi]_{X}: x \in X\right\}}
\end{gathered}
$$

for all variables $x, \ddot{x}$, predicate symbols $p \in \Sigma$, and formulas $\varphi, \psi$ of signature $\Sigma$, where $\neg_{B}$ is the complement operation in B. The above definitions are conditionally correct (see Agreement 4) provided that the parameters of the formulas under consideration belong to $X$.

Equalities (2) correctly define the function symbols $\langle\varphi\rangle_{X}$ within ZFC under the hypothesis " $X$ is a $B$-presystem." According to Definition 5 and Section 2.4, the definitions lead to a conservative extension of ZFC that proves $(\forall \ddot{x} \in X)[\varphi(\ddot{x})]_{X} \in B$ for each formula $\varphi$ of signature $\Sigma$.

Strictly speaking, the parameters of the term $[\varphi]_{X}$ are not only those of the formula $\varphi$, but also the parameters of the hypotheses declared in the definition of Boolean truth values (including the variable $B$ and the optional parameters of the classes $X$ and $[p]_{X}$ ). Nevertheless, with account taken of Propositions 3 or 4 , the parameters of hypotheses can be regarded as constants and excluded from the arguments of the function symbols $\langle\varphi\rangle_{\mathrm{X}}$.

Lemma 1. Let $X$ be a B-presystem of signature $\Sigma$, let $\varphi(\ddot{x})$ be a formula of signature $\Sigma$ with parameters $\ddot{x}$, and let $\ddot{y}$ be an arbitrary list of variables with $|\ddot{y}|=|\ddot{x}|$. Then (the extension of) ZFC proves that, for all $\ddot{y} \in X$,

(a) $\left.[\varphi]_{X}\right|_{\dddot{y}} ^{\dddot{x}}=\left[\left.\varphi\right|_{\dddot{y}} ^{\dddot{x}}\right]_{X} ;$

(b) $\langle\varphi(\ddot{x})\rangle_{X}(\ddot{y})=[\varphi(\ddot{y})]_{X}$.

Proof. (a): Consider the case of an atomic formula, $p(\ddot{x})$, with $p \in \Sigma$. According to definitions (2), for all $\ddot{x}, \ddot{y} \in X$ we have $[p(\ddot{x})]_{X}=[p]_{X}(\ddot{x}),[p(\ddot{y})]_{X}=[p]_{X}(\ddot{y})$, and, consequently,

$$
\left.[p(\dddot{x})]_{X}\right|_{\dddot{y}} ^{\dddot{x}}=\left.[p]_{X}(\ddot{x})\right|_{\dddot{y}} ^{\dddot{x}}=[p]_{X}(\ddot{y})=[p(\dddot{y})]_{X}=\left[\left.p(\ddot{x})\right|_{\dddot{y}} ^{\dddot{x}}\right]_{X} .
$$

The case of a complex formula $\varphi$ is easily proven by induction on the complexity.

(b): Due to equality (a) we have

$$
\langle\varphi(\ddot{x})\rangle_{X}(\dddot{y})=\left.\langle\varphi(\ddot{x})\rangle_{X}(\dddot{x})\right|_{\ddot{y}} ^{\dddot{x}}=\left.[\varphi(\dddot{x})]_{X}\right|_{\ddot{y}} ^{\dddot{x}}=\left[\left.\varphi(\dddot{x})\right|_{\dddot{y}} ^{\dddot{x}}\right]_{X}=[\varphi(\dddot{y})]_{X} .
$$

From equality (b) it follows that the expressive power of the language will not decrease if we refuse to employ expressions of the form $\langle\varphi\rangle_{X}(\ldots)$ and confine ourselves to the use of terms $[\varphi]_{X}$ (cp. Definitions 6 and 7).

Definition 12. Let $X$ be a B-presystem of signature $\Sigma$ and let $\varphi(\ddot{x})$ be a formula of signature $\Sigma$ with parameters $\ddot{x}$. On assuming $\ddot{x} \in X$, say that $\varphi(\ddot{x})$ is valid in $X$ and write

$$
X \vDash \varphi(\ddot{x})
$$


provided that $[\varphi(\ddot{x})]_{X}=1_{B}$. Given a set $\mathcal{F}$ of sentences of signature $\Sigma$, say that $\mathcal{F}$ is valid in $X$ and write $X \vDash \mathcal{F}$ whenever $X \vDash \varphi$ for all $\varphi \in \mathcal{F}$.

Remark 5. Since the article is devoted to the study of Boolean-valued systems of set-theoretic signature (that is constituted by predicate symbols only), we considered it appropriate to simplify the exposition by excluding function symbols from Definitions 10 and 11. It is worth noting that the traditional approach, in which $n$-ary function symbols $f$ in a system $X$ are interpreted by functions $[f]_{X}: X^{n} \rightarrow X$, is not the most general solution in the Boolean-valued case. Indeed, in this approach, for all $\ddot{x}=x_{1}, \ldots, x_{n} \in X$, the condition

$$
X \vDash(\exists y)(f(\ddot{x})=y)
$$

is fulfilled in a considerably stronger form,

$$
(\exists y \in X) X \vDash(f(\ddot{x})=y),
$$

which automatically provides the maximum principle for the formula $(\exists y)(f(\dddot{x})=y)$ (see Definition 43). A less restrictive approach consists in considering a predicate symbol $f^{*}$ of arity $n+1$ with interpretation subject to

$$
X \vDash(\forall \ddot{x})(\exists ! y) f^{*}(\ddot{x}, y)
$$

and then employing the eliminable extension in which the function symbol $f$ is defined via $f^{*}$ by the axiom

$$
f(\ddot{x})=y \Leftrightarrow f^{*}(\ddot{x}, y) .
$$

Anyway, such a generalization is unnecessary for the present article.

Definition 13. Let $X$ be a B-presystem of a signature with equality. Say that $\left(X,[=]_{X}\right)$ is a Bmodel of equality if for all $x, y, z \in X$

$$
\begin{aligned}
& {[x=x]_{X}=1_{B} ;} \\
& {[x=y]_{X}=[y=x]_{X} ;} \\
& {[x=y]_{X} \wedge_{B}[y=z]_{X} \leqslant{ }_{B}[x=z]_{X}}
\end{aligned}
$$

or, which is the same, the axioms of equality for signature $\{=\}$ are valid in $X$ :

$$
X \vDash(\forall \ldots)((x=x) \wedge(x=y \Rightarrow x=y) \wedge(x=y \wedge y=z \Rightarrow x=z)) .
$$

Let $\left(X,[=]_{X}\right)$ be a $B$-model of equality.

Proposition 5. The following properties of a function $\Phi: X \rightarrow B$ are equivalent:

(a) $\Phi(x) \wedge_{B}[x=y]_{X} \leqslant{ }_{B} \Phi(y)$ for all $x, y \in X$;

(b) $\Phi(x) \wedge_{B}[x=y]_{X}=\Phi(y) \wedge_{B}[x=y]_{X}$ for all $x, y \in X$;

(c) $[x=y]_{X} \geqslant_{B} b \Rightarrow \Phi(x) \wedge_{B} b=\Phi(y) \wedge_{B} b$ for all $x, y \in X, b \in B$;

(d) $[x=y]_{X} \leqslant B\left(\Phi(x) \Leftrightarrow_{B} \Phi(y)\right)$ for all $x, y \in X$, where $\left(a \Leftrightarrow_{B} b\right)=\left(\neg_{B} a \vee_{B} b\right) \wedge_{B}\left(\neg_{B} b \vee_{B} a\right)$.

Definition 14. A function $\Phi: X \rightarrow B$ subject to each of the equivalent conditions (a)-(d) of Proposition 5 is called extensional; see [1] (3.5), [4] (2.5.5), [5] (4.5.6). Say that a function $\Phi: X^{n} \rightarrow B$ is extensional if $\Phi$ is extensional in every of the $n$ arguments, which is equivalent to each of the following four conditions (see Definition 3):

$$
\begin{aligned}
& \Phi(\ddot{x}) \wedge_{B}[\ddot{x}=\ddot{y}]_{X} \leqslant{ }_{B} \Phi(\ddot{y}) ; \\
& \Phi(\ddot{x}) \wedge_{B}[\ddot{x}=\ddot{y}]_{X}=\Phi(\ddot{y}) \wedge_{B}[\ddot{x}=\dddot{y}]_{X} ; \\
& {[\ddot{x}=\dddot{y}]_{X} \geqslant_{B} b \Rightarrow \Phi(\ddot{x}) \wedge_{B} b=\Phi(\ddot{y}) \wedge_{B} b ;} \\
& {[\ddot{x}=\ddot{y}]_{X} \leqslant B\left(\Phi(\ddot{x}) \Leftrightarrow_{B} \Phi(\ddot{y})\right)}
\end{aligned}
$$


for all $\ddot{x}=x_{1}, \ldots, x_{n} \in X, \dddot{y}=y_{1}, \ldots, y_{n} \in X, b \in B$.

Extensional functions $\Phi: X \rightarrow B$ are also called Boolean-valued classes in X (see [1] (3.5), [4] (2.5.8), [5] (4.6.1)) and are employed in the language of truth values in a manner similar to the use of classes in the language of set theory (see Definition 22 below).

In the sequel, the assertion that $\Phi: X \rightarrow B$ is a Boolean-valued class (i.e., $\Phi$ is extensional) will be written as $\Phi \Subset X$.

Let $\Sigma$ be a predicative signature with equality.

Proposition 6. The following properties of a B-presystem $X$ of signature $\Sigma$ are equivalent:

(a) $\left(X,[=]_{X}\right)$ is a B-model of equality and the interpretations $[p]_{X}: X^{n} \rightarrow B$ of all the symbols $p \in \Sigma \backslash\{=\}$ are extensional;

(b) the axioms of equality for signature $\Sigma$ are valid in $X$ :

$$
\begin{aligned}
& X \vDash(\forall \ldots)((x=x) \wedge(x=y \Rightarrow x=y) \wedge(x=y \wedge y=z \Rightarrow x=z)) ; \\
& X \vDash(\forall \ldots)(p(\ddot{x}) \wedge \ddot{x}=\ddot{y} \Rightarrow p(\ddot{y})) \text { for each symbol } p \in \Sigma \backslash\{=\} ;
\end{aligned}
$$

(c) $X \vDash(\forall . ..) \varphi$ for each axiom $\varphi$ of predicate calculus of signature $\Sigma$ (with equality);

(d) $X \vDash(\forall . ..) \varphi$ for all formulas $\varphi$ deducible in predicate calculus of signature $\Sigma$;

(e) all closed tautologies of signature $\Sigma$ are valid in $X$.

Whenever the equivalent conditions (a)-(e) hold, say that $X$ is a Boolean-valued (more exactly, B-valued) algebraic system of signature $\Sigma$. We will also use the shorter synonyms: Boolean-valued system and B-system of signature $\Sigma$.

The following simple consequence of condition (d) will be often used without explicit reference:

Proposition 7. Let $X$ be a B-system of signature $\Sigma$ and let $\varphi$ be a formula of signature $\Sigma$ with parameters $x_{1}, \ldots, x_{n}$. Then the following function is extensional:

$$
\left(x_{1}, \ldots, x_{n}\right) \in X^{n} \mapsto\left[\varphi\left(x_{1}, \ldots, x_{n}\right)\right]_{X} \in B .
$$

As is known, the deduction rules preserve validity in any Boolean-valued system. More exactly, the following holds:

Proposition 8. Let $X$ be a B-system of signature $\Sigma$ and let $\Gamma$ and $\Delta$ be some sets of sentences of signature $\Sigma$.

(a) If $\vdash \Delta$ then $\mathrm{ZFC} \vdash(X \vDash \Delta)$.

(b) If $\mathrm{ZFC} \vdash(X \vDash \Gamma)$ and $\Gamma \vdash \Delta$ then $\mathrm{ZFC} \vdash(X \vDash \Delta)$.

Therefore, if $\operatorname{Th}(\mathcal{T})$ is the totality of all theorems of a theory $\mathcal{T}$, the assertions $X \vDash(\forall \ldots) \mathcal{T}$ and $X \vDash(\forall \ldots) \operatorname{Th}(\mathcal{T})$ are equivalent. In each of the cases we call $X$ a Booleanvalued model (or a B-model) of $\mathcal{T}$ and write $X \vDash \mathcal{T}$. Assertions (c) and (d) of Proposition 6 correspond to the case $\mathcal{T}=\varnothing$ and state that every $B$-system is a Boolean-valued model of predicate calculus.

Definition 15. Let $X$ be a B-system of signature $\Sigma$. Consider the following equivalence $\sim$ on $X$ :

$$
x \sim y \Leftrightarrow[x=y]_{X}=1_{B} \Leftrightarrow X \vDash(x=y) .
$$

The system $X$ is called separated if $x \sim y \Leftrightarrow x=y$ for all $x, y \in X$. In the case of a 2-system, where $2:=\{0,1\}$ is the simplest Boolean algebra; $X$ is separated whenever the interpretation of equality in $X$ is standard: $[=]_{X}(x, y)=1 \Leftrightarrow x=y$. 
The quotient $\widetilde{X}:=X / \sim($ see [4] (2.5), [5] (4.5)) is a separated B-system that is elementary equivalent to the initial system: $[\varphi]_{\tilde{X}}=[\varphi]_{X}$ for every sentence $\varphi$ of signature $\Sigma$. Moreover, for each formula $\varphi$ of signature $\Sigma$ with parameters $x_{1}, \ldots, x_{n}$, we have

$$
\left[\varphi\left(y_{1}, \ldots, y_{n}\right)\right]_{\tilde{X}}=\left[\varphi\left(x_{1}, \ldots, x_{n}\right)\right]_{X}
$$

whenever $x_{1}, \ldots, x_{n} \in X$ and $y_{i}=\tilde{x}_{i} \in \tilde{X}$ are the cosets of $x_{i}$.

Definition 16. Given a B-system $X$ of signature $\Sigma$, a B-system $Y$ is called a subsystem of $X$ if $Y \subset X$ and $[p]_{Y}=\left.[p]_{X}\right|_{Y}$ for all $p \in \Sigma$.

Definition 17. Say that a class $f$ is an isomorphism between B-systems $X$ and $Y$ of signature $\Sigma$ and write $f: X \leftrightarrow_{B} Y$, if $f$ is a bijection between $X$ and $Y$ subject to the condition

$$
\left(\forall x_{1}, \ldots, x_{n} \in X\right)[p]_{X}\left(x_{1}, \ldots, x_{n}\right)=[p]_{Y}\left(f\left(x_{1}\right), \ldots, f\left(x_{n}\right)\right)
$$

for each $n$-ary symbol $p \in \Sigma$. As is easily seen, if $\varphi$ is a formula of signature $\Sigma$ with parameters $x_{1}, \ldots, x_{n}$, then $f: X \leftrightarrow_{B} Y$ implies

$$
\begin{aligned}
& \left(\forall x_{1}, \ldots, x_{n} \in X\right)\left(\forall y_{1}, \ldots, y_{n} \in Y\right) \\
& \left(f\left(x_{1}\right)=y_{1} \wedge \cdots \wedge f\left(x_{n}\right)=y_{n} \Rightarrow\left[\varphi\left(x_{1}, \ldots, x_{n}\right)\right]_{X}=\left[\varphi\left(y_{1}, \ldots, y_{n}\right)\right]_{Y}\right) .
\end{aligned}
$$

Say that B-systems $X$ and $Y$ are isomorphic and write $X \leftrightarrow_{B} Y$ whenever $Z F C \vdash\left(f: X \leftrightarrow_{B} Y\right)$ for some class $f$.

\subsection{Eliminable Extensions in Truth Values}

According to Definition 11, given a Boolean-valued model $X$ of set theory, the terms $[\varphi]_{X}$ make sense for formulas $\varphi$ of signature $\{=, \in\}$ but not for formulas containing any additional definable predicate or function symbols. In this subsection, we discuss and justify the convenient use of such expressions as $[x \subset y]_{X^{\prime}}[x \cap y=\varnothing]_{X^{\prime}}$ or $[f: \mathbb{R} \rightarrow \mathbb{R}]_{X}$. We also formalize the use of outer terms in the language of truth values by means of an eliminable extension that makes it possible to delegate the semantics of a term to the outer theory and legalizes the expressions of the form $[\ldots \underline{\tau} \ldots]_{X}$, with $\tau$ a term defined in ZFC, even for the case in which $X$ is not a model of set theory.

Definition 18. Let $X$ be a B-system of signature $\Sigma$, let $\mathcal{T}$ be a theory of signature $\Sigma$, let $\mathcal{T}^{*}$ be an eliminable extension of $\mathcal{T}$ of an arbitrary (not necessarily predicative) signature $\Sigma^{*}$, and let $\lceil\cdot\rceil: F\left(\Sigma^{*}\right) \rightarrow F(\Sigma)$ be the corresponding elimination (see Agreement 5). Acting in a similar way to Definition 11, for each formula $\varphi \in F\left(\Sigma^{*}\right) \backslash F(\Sigma)$ with parameters $\ddot{x}$, enrich the signature of ZFC by the new function symbol $\langle\varphi\rangle_{X}$ of arity $|\ddot{x}|$, introduce the notation $[\varphi]_{X}:=\langle\varphi(\ddot{x})\rangle_{X}(\ddot{x})$, and add the defining axiom

$$
[\varphi]_{X}=[\lceil\varphi\rceil]_{X}
$$

to ZFC. Note that the extended syntax of Boolean truth values remains invariant with respect to the logical connectives: the equalities (2) occur provable for the formulas $\varphi, \psi$ of the enriched signature $\Sigma^{*}$.

The validity in $X$ for the new formulas $\varphi$ of signature $\Sigma^{*}$ is defined by the conventional relation $X \vDash \varphi \Leftrightarrow[\varphi]_{X}=1_{B}$.

The corresponding grammatical closure (cp. Remark 3) enriches the language by terms of the form $\left[\ldots[\varphi]_{Y} \ldots\right]_{X}$ and thus makes it possible to consider Boolean-valued models inside Booleanvalued models (see, e.g., [1]).

Definition 18 makes it possible to regard every Boolean-valued model of a theory $\mathcal{T}$ as a model of an arbitrary eliminable extension $\mathcal{T}^{*}$ of $\mathcal{T}$. Namely, the following holds: 
Proposition 9. Let $X$ be a B-model of a theory $\mathcal{T}$ of signature $\Sigma$, and let $\mathcal{T}^{*}$ be an eliminable extension of $\mathcal{T}$ of signature $\Sigma^{*}$.

(a) The system $X$ is a model of $\mathcal{T}^{*}$ in the sense that $X \vDash(\forall ..) \varphi$ for each theorem $\varphi$ of $\mathcal{T}^{*}$.

(b) If the signature $\Sigma^{*}$ is predicative, then the truth value $[\varphi]_{X}$ of every formula $\varphi \in F\left(\Sigma^{*}\right)$ coincides with its truth value $[\varphi]_{X^{*}}$ in the class $X$ regarded as a B-valued system of signature $\Sigma^{*}$ with interpretations

Moreover, $X^{*} \vDash \mathcal{T}^{*}$.

$$
[p]_{X^{*}}(\ddot{x})=[\lceil p(\dddot{x})\rceil]_{X^{\prime}}, \quad p \in \Sigma^{*} .
$$

Remark 6. The field of reals $\mathbb{R}$ is often defined as an arbitrary Dedekind-complete totally ordered field. In ZFC, such an ordered field is unique up to isomorphism, but not unique; therefore, the corresponding definition of the constant $\mathbb{R}$ does not meet the conditions of Definition 5. In more detail, let $\delta(x)$ be a formalization, in the language of ZFC, of the assertion " $x$ is a Dedekind-complete totally ordered field," and let $\mathrm{ZFC}_{\mathbb{R}}$ be the extension of $\mathrm{ZFC}$ by the constant $\mathbb{R}$ and the axiom $\delta(\mathbb{R})$.

Proposition 10. If $\mathrm{ZFC}$ is consistent, then the extension $\mathrm{ZFC} \subset \mathrm{ZFC} \mathbb{R}_{\mathbb{R}}$ is not eliminable.

Proof. It suffices to observe that the formula $x=\mathbb{R}$ cannot be eliminated. Indeed, if there was a formula $\varepsilon(x)$ of signature $\{=, \in\}$ such that $\mathrm{ZFC}_{\mathbb{R}} \vdash(x=\mathbb{R} \Leftrightarrow \varepsilon(x))$, then we would have ZFC $\vdash(\delta(r) \Rightarrow(\forall x)(x=r \Leftrightarrow \varepsilon(x)))$ and, in particular, ZFC $\vdash\left(\delta\left(r_{1}\right) \wedge \delta\left(r_{2}\right) \Rightarrow\right.$ $\left.r_{1}=r_{2}\right)$, which is not the case.

However, due to the provability of $(\exists x) \delta(x)$ in ZFC, the theory $Z_{F} C_{\mathbb{R}}$ occurs a conservative extension of ZFC. Moreover, the extension $Z F C \subset Z F C_{\mathbb{R}}$ admits an elimination in the following weaker sense: every formula $\varphi$ of signature $\{=, \in, \mathbb{R}\}$ can be computably associated with a formula $\lceil\varphi\rceil$ of signature $\{=, \in\}$ such that

$$
\mathrm{ZFC}_{\mathbb{R}} \vdash \varphi \Leftrightarrow \mathrm{ZFC} \vdash\lceil\varphi\rceil .
$$

The role of $\lceil\varphi\rceil$ can be played by the implication $\left.\delta(r) \Rightarrow \varphi\right|_{r} ^{\mathbb{R}}$, where $\left.\varphi\right|_{r} ^{\mathbb{R}}$ is the result of replacing all occurrences of the constant $\mathbb{R}$ in $\varphi$ with a new variable $r$.

Therefore, the definition of the constant $\mathbb{R}$ as "an arbitrary Dedekind-complete totally ordered field" is formalized by a conservative but not eliminable extension. The lack of elimination complicates modeling the extension in a Boolean-valued model X of ZFC (see Definition 18). In the case under consideration, instead of embedding the constant $\mathbb{R}$ inside the syntax of $[\ldots]_{X}$, the symbol $\mathcal{R}$ is introduced as "an arbitrary element of $X$ that is a Dedekind-complete totally ordered field inside $X$. .' This approach is formalized by adding the constant $\mathcal{R}$ and the axiom

$$
(\exists x \in X)(x=\mathcal{R} \wedge X \vDash \delta(x)) .
$$

The resultant theory occurs again a conservative but not eliminable extension of ZFC.

Remark 7. The constant $\mathbb{R}$ becomes eliminable if we choose a "concrete" definition of the ordered field of reals (for instance, as the set of decimal fractions or continued fractions, or as the set of cosets of Cauchy sequences, etc.), that is, a description that provides the uniqueness of the object under definition.

For example, let $\rho(x)$ be a formalization, in the language of $\mathrm{ZFC,} \mathrm{of} \mathrm{the} \mathrm{assertion} \mathrm{that} x$ is the set of all Dedekind cuts. Employing the formalism of Definition 5, define the constant $\mathbb{R}$ by the axiom $x=\mathbb{R} \Leftrightarrow \rho(x)$. The symbol $\mathbb{R}$ now becomes an element of the formal language of the extended theory. Since ZFC $\vdash(\exists ! x) \rho(x)$, the resultant extension is eliminable (see Theorem 1), and we now have the possibility of extending the syntax of truth values $[\ldots]_{X}$ in a Boolean-valued model $X$ of ZFC onto the formulas $\varphi$ of the extended signature by putting $[\varphi]_{X}=[\lceil\varphi\rceil]_{X}$ (see Definition 18). As a result, we obtain an eliminable extension, $Z \mathrm{ZFC}^{*}$, and $\mathrm{X}$ occurs a model of the theory.

Since ZFC proves the translation $(\exists ! x) \rho(x)$ of the formula $(\exists ! x)(x=\mathbb{R})$, the latter is valid in every Boolean-valued model $X$ of ZFC. In particular, if $X$ is separated and satisfies the maximum principle (see Definitions 15 and 43), there exists a unique $x \in X$ subject to the condition $X \vDash$ 
$(x=\mathbb{R})$. Therefore, each such model has a unique element that "is $\mathbb{R}$ " inside the model. The symbol $\mathbb{R}$ not only "names the element $x \in \mathbb{V}$ subject to the definition $\rho(x)$," but also serves as a universal name for the field of reals in all models of ZFC.

The universality of the constant described is not surprising, since the symbol belongs to the signature of the language of the theory under consideration rather than to a model of the theory. In this respect, the constant $\mathbb{R}$ does not stand out from the other elements of the signature, including the predicate symbol $\in$ that occurs in expressions $[\ldots]_{X}$ and $X \vDash(\ldots)$ without any special syntactic modifications. So, the formula $X \vDash(x \in y)$ has translation $\lceil X \vDash(x \in y)\rceil=\left\lceil[\in]_{X}(x, y)=1_{B}\right\rceil$ into the initial language of set theory as a formula with parameters $x, y$. In exactly the same way, the formula $X \vDash(x=\mathbb{R})$ has translation $\lceil X \vDash(x=\mathbb{R})\rceil=\lceil X \vDash\lceil x=\mathbb{R}\rceil\rceil=\lceil X \vDash \rho(x)\rceil=$ $\left\lceil[\rho(x)]_{X}=1_{B}\right\rceil$ as a formula with parameter $x$.

Remark 8. A possible discomfort brought by the expression $X \vDash(x=\mathbb{R})$ is caused not so much by the universal use of the constant $\mathbb{R}$ as by inconsistency of the syntax of truth values with term substitution: the formula $X \vDash(x=\mathbb{R})$ is not equivalent to the result $\left.(X \vDash(x=y))\right|_{\mathbb{R}} ^{y}$ of substitution $\mathbb{R}$ for $y$ in $X \vDash(x=y)$. Indeed, in the case of a separated model we have $X \vDash$ $(x=y) \Leftrightarrow x=y$ and hence

whereas

$$
\left.\left.(X \vDash(x=y))\right|_{\mathbb{R}} ^{y} \Leftrightarrow(x=y)\right|_{\mathbb{R}} ^{y} \Leftrightarrow x=\mathbb{R} \Leftrightarrow \rho(x),
$$

$$
X \vDash(x=\mathbb{R}) \Leftrightarrow X \vDash\lceil x=\mathbb{R}\rceil \Leftrightarrow X \vDash \rho(x) .
$$

The phenomenon is caused by the lack of the identity $\left.[\varphi]_{X}\right|_{\tau} ^{y}=\left[\left.\varphi\right|_{\tau} ^{y}\right]_{X}$ for the case in which the term $\tau$ is not a variable. Moreover, if the signature $\Sigma$ of the system X differs from $\{=, \in\}$, then the substitution $\left.\varphi\right|_{\tau} ^{y}$ does not make sense at all: the formula $\varphi$ belongs to the language of $\Sigma$, while the term $\tau$ belongs to the language of set theory. The truth valuation and term substitution commute only in the case of the simplest terms, the variables (see Lemma 1(a)).

$B y$ definition, the validity $X \vDash \varphi$ amounts to $[\lceil\varphi\rceil]_{X}=1_{B}$; hence, elimination of the definable symbols involved in $\varphi$ is performed "inside" the construction $[\ldots]_{X}$ and is not delegated outside its syntactic margins. This circumstance adequately reflects the concept of modeling: Being a model of set theory, $X$ interprets in its own way not only the basic predicates $=$ and $\in$, but also all symbols defined in the theory, including the predicate $\subset$, constants $\varnothing, \mathbb{N}, \mathbb{R}$, function symbols $\mathcal{P}, \cup$, and so forth. So, as soon as we know that the validity of the assertion " $x$ consists of all subsets of $y$ " depends on the model in which it is verified, we easily agree that the formulas $x=\mathcal{P}(y)$ and $X \vDash(x=\mathcal{P}(y))$ can be nonequivalent; for a similar reason, the universality of the constant $\mathbb{R}$ and the difference between the formula $X \vDash(x=\mathbb{R})$ and the substitution $\left.(X \vDash(x=y))\right|_{\mathbb{R}} ^{y}$ become commonplace, if we take account of the fact that $\mathbb{R}$ is a function symbol like $\mathcal{P}$ and only differs in arity.

Remark 9. The definition of the canonical embedding ${ }^{\wedge}: \mathbb{V} \hookrightarrow \mathbb{V}^{(B)}$ (see [4] (2.2.7), [5] (4.2.7)) as a unary function symbol ${ }^{\wedge}$ is conventionally accompanied by the agreement on denoting the standard name $^{\wedge}(x)$ of $x$ as $x^{\wedge}$. Despite the fact that the definition $x^{\wedge}=\left\{y^{\wedge}: y \in x\right\} \uparrow$ is recursive, it results in an eliminable extension of ZFC; therefore, according to Definition 5 , the equality $y=x^{\wedge}$ admits a translation into the language of set theory as a formula $\lambda(x, y)$.

By saying " $y$ is a real" we mean $y \in \mathbb{R}$, and the phrase " $y$ is a real inside $\mathbb{V}^{(B) \text { " means }}$ $\mathbb{V}^{(B)} \vDash(y \in \mathbb{R})$. One might think that the phrase " $x^{\wedge}$ is a real inside $\mathbb{V}^{(B)}$ " is adequately expressed by the formula $\mathbb{V}^{(B)} \vDash\left(x^{\wedge} \in \mathbb{R}\right)$, but the experience accumulated in Remark 8 suggests that this is not the case. Since the definable symbols are subject to elimination inside the truth value construction, the formula $\mathbb{V}^{(B)} \vDash\left(x^{\wedge} \in \mathbb{R}\right)$ expresses the assertion that, inside $\mathbb{V}^{(B)}$, the reals contain the standard name $x^{\wedge}$ calculated inside $\mathbb{V}^{(B)}$ :

$$
\mathbb{V}^{(B)} \vDash\left(x^{\wedge} \in \mathbb{R}\right) \Leftrightarrow \mathbb{V}^{(B)} \vDash(\exists y)\left(y=x^{\wedge} \wedge y \in \mathbb{R}\right) \Leftrightarrow \mathbb{V}^{(B)} \vDash(\exists y)(\lambda(x, y) \wedge y \in \mathbb{R}) .
$$

On the other hand, by saying " $x^{\wedge}$ is a real inside $\mathbb{V}^{(B) "}$ " we assume calculation of $x^{\wedge}$ outside $\mathbb{V}^{(B)}$ and actually have in mind

$$
\left.\left(\mathbb{V}^{(B)} \vDash(y \in \mathbb{R})\right)\right|_{x^{\wedge}} ^{y} \Leftrightarrow(\exists y)\left(y=x^{\wedge} \wedge \mathbb{V}^{(B)} \vDash(y \in \mathbb{R})\right) \Leftrightarrow(\exists y)\left(\lambda(x, y) \wedge \mathbb{V}^{(B)} \vDash(y \in \mathbb{R})\right) .
$$


The above formalization is rather bulky. The following approach makes it possible to combine formality and brevity.

Definition 19. Let $X$ be an arbitrary B-system of signature $\Sigma$. Consider the signature $\Sigma$ obtained from $\Sigma$ by adding the constant $\underline{\tau}$ for each set-theoretic term $\tau$. The constants $\underline{\tau}$ are called outer terms. We will define the Boolean truth values for the formulas of signature $\underline{\Sigma}$ in such a manner that outer terms will be evaluated "outside the Boolean-valued system." Namely, we introduce the terms $[\psi]_{X}, \psi \in F(\underline{\Sigma})$, as follows: Given a formula $\varphi(\ddot{x}, \ddot{y})$ of signature $\Sigma$ whose parameters are arbitrarily partitioned into two parts, $\ddot{x}=x_{1}, \ldots, x_{m}$ and $\ddot{y}=y_{1}, \ldots, y_{n}$, and given arbitrary set-theoretic terms $\ddot{\tau}=\tau_{1}, \ldots, \tau_{n}$; consider the parameters $\dddot{z}$ of the set $\ddot{x} \cup \dddot{\tau}$ (see Definition 2); enrich the language of ZFC by the function symbol $\langle\varphi(\ddot{x}, \underline{\tilde{\tau}})\rangle_{X}$ of arity $|\ddot{z}|$, with $\underline{\underline{\tau}}=\underline{\tau_{1}}, \ldots, \underline{\tau_{n}}$; introduce the notation $[\varphi(\ddot{x}, \underline{\tilde{\tau}})]_{X}:=\langle\varphi(\ddot{x}, \underline{\tau})\rangle_{X}(\ddot{z})$; and extend ZFC by the defining axiom

$$
[\varphi(\dddot{x}, \underline{\tau})]_{X}:=\left.[\varphi(\dddot{x}, \dddot{y})]_{X}\right|_{\dddot{\tau}} ^{\dddot{y}}=\langle\varphi(\dddot{x}, \dddot{y})\rangle_{X}(\dddot{x}, \dddot{\tau})
$$

or, which is equivalent, by the axiom

$$
\begin{aligned}
b=\left[\varphi\left(\ddot{x}, \underline{\tau_{1}}, \ldots, \underline{\tau_{n}}\right)\right]_{X} \Leftrightarrow\left(\exists y_{1}, \ldots, y_{n}\right)\left(y_{1}\right. & =\tau_{1} \wedge \cdots \wedge y_{n}=\tau_{n} \\
& \left.\wedge b=\left[\varphi\left(\ddot{x}, y_{1}, \ldots, y_{n}\right)\right]_{X}\right) .
\end{aligned}
$$

The validity $X \vDash \varphi(\dddot{x}, \underline{\tilde{\tau}})$ is conventionally defined as the equality $[\varphi(\dddot{x}, \underline{\underline{\tau}})]_{X}=1_{B}$.

The use of outer terms considerably simplifies syntax constructions while keeping

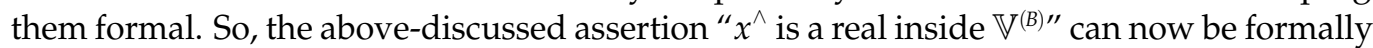
written as

$$
\mathbb{V}^{(B)} \vDash\left(\underline{x^{\wedge}} \in \mathbb{R}\right),
$$

and the property (3) of an isomorphism $f: X \leftrightarrow_{B} Y$ takes the more concise form

$$
\left(\forall x_{1}, \ldots, x_{n} \in X\right)\left[\varphi\left(x_{1}, \ldots, x_{n}\right)\right]_{X}=\left[\varphi\left(\underline{f\left(x_{1}\right)}, \ldots, \underline{f\left(x_{n}\right)}\right)\right]_{Y} .
$$

Outer terms are used in mathematical practice without any special syntax. This departure from formalism is conventionally compensated by the context. For instance, in the presence of subsets $X, Y \subset \mathbb{V}^{(B)}$, an extensional mapping $f: X \rightarrow Y$, and an element $x \in X$; it is easy to find out that the expression $\mathbb{V}^{(B)} \vDash(f \uparrow(x)=f(x))$ (see [4] (3.3.11), [5] (5.5.6)) employs the outer terms $f \uparrow$ and $f(x)$ : in the context under consideration, the formula

$$
\mathbb{V}^{(B)} \vDash(\underline{f \uparrow}(x)=\underline{f(x)})
$$

is actually implied, which is equivalent to $(\exists g)(\exists y)\left(g=f \uparrow \wedge y=f(x) \wedge \mathbb{V}^{(B)} \vDash(g(x)=y)\right)$. Following the tradition, we will not underline outer terms in the sequel.

\subsection{Classes in Truth Values}

In this subsection, we extend the syntax of Boolean truth values by definable (internal) classes and, which is more important, by external Boolean-valued classes. To make the latter possible, we first describe the general machinery of extending a theory by means of external classes. Those are undefined unary predicates supplemented with a syntactic sugar that turns them into constants. Next, arbitrary Boolean-valued classes $\Phi$ are associated with the corresponding unary predicate symbols $\operatorname{Sym}(\Phi)$ that are interpreted by $\Phi$ and, therefore, can be used in the language of truth values.

Definition 20. The language of Boolean truth values is extended by the use of internal classes in much the same manner as in Definition 18. Namely, let $\epsilon$ be the standard set-theoretic signature $\{=, \in\}$, and let $\mathrm{cl}^{\omega} \in$ be the enrichment of $\in$ by the symbols of internal classes (see Definition 7). If $X$ is a Boolean-valued system of signature $\epsilon$, then, given a formula $\varphi$ of signature $\mathrm{cl}^{\omega} \epsilon$, the truth value $[\varphi]_{X}$ is defined as $[\lceil\varphi\rceil]_{X}$, where $\lceil\cdot\rceil: F\left(\mathrm{cl}^{\omega} \boldsymbol{\epsilon}\right) \rightarrow F(\boldsymbol{\epsilon})$ is the elimination of classes. 
As in Definition 7, if definable classes are used inside a Boolean-valued model together with other symbols defined by means of an eliminable extension, then, in order to calculate the truth value $[\varphi]_{X}$, we should first apply an elimination of the extension to $\varphi$, and then apply the elimination of classes.

Agreement 7. Such frequently used terms as $\varnothing,\{x\}$, or $x \cap y$ are defined by means of an eliminable extension of ZFC, but their conventional definitions are not correct within the pure predicate calculus of signature $\epsilon$ without any special axioms; therefore, Definition 18 is not sufficient for making the expressions $[x \neq \varnothing]_{X},[y=\{x\}]_{X}$ or $[x \cap y=z]_{X}$ sensible in the case of an arbitrary Boolean-valued system $X$ of signature $\epsilon$. We can give them sense by the agreement to interpret the terms $\varnothing,\{x\}$, and $x \cap y$ as the names of definable classes:

$$
\begin{aligned}
\varnothing & =\{u: u \neq u\}, \\
\{x\} & =\{u: u=x\}, \\
x \cap y & =\{u: u \in x \wedge u \in y\} .
\end{aligned}
$$

Then, according to Definitions 7 and 20, we have

$$
\begin{aligned}
{[x \neq \varnothing]_{X} } & =[\neg(\forall u)(u \in x \Leftrightarrow u \in \varnothing)]_{X}=[\neg(\forall u)(u \in x \Leftrightarrow u \neq u)]_{X}=[(\exists u)(u \in x)]_{X}, \\
{[y=\{x\}]_{X} } & =[(\forall u)(u \in y \Leftrightarrow u \in\{x\})]_{X}=[(\forall u)(u \in y \Leftrightarrow u=x)]_{X}, \\
{[x \cap y=z]_{X} } & =[(\forall u)(u \in x \cap y \Leftrightarrow u \in z)]_{X}=[(\forall u)(u \in x \wedge u \in y \Leftrightarrow u \in z)]_{X} .
\end{aligned}
$$

We will repeatedly use the above agreement (see, e.g., Lemmas 2, 5, 9, 11 and 13).

The extension of the language of set theory by the syntax of external classes plays an important role in the theory of Boolean-valued models. The extension consists in the addition of new predicate symbols that are grammatically used as function symbols. We will describe the formalism for the case of external classes without parameters.

Definition 21. Let $\epsilon$ be the standard set-theoretic signature $\{=, \in\}$, and let $\epsilon^{*}$ be the enrichment of $\epsilon$ by a set $\mathcal{P}$ of unary predicate symbols. Enrich $\epsilon^{*}$ to the signature $\epsilon_{\mathrm{c}}^{*}$ by adding, for each symbol $C \in \mathcal{P}$, the corresponding external class, the constant (i.e., 0-ary function symbol) with the same name $C$. (The definition of signature usually requires that the sets of predicate and function symbols do not intersect; however, in the case under consideration, predicates and constants with equal names are easily distinguished due to different grammatical roles and arities. For example, it is clear that, in the formula $C(C)$, the first occurrence of $C$ is a predicate, while the second is a constant.)

As is easily seen, there exists a unique mapping

$$
\lceil\cdot\rceil: F\left(\epsilon_{\mathrm{c}}^{*}\right) \rightarrow F\left(\epsilon^{*}\right)
$$

that is identical on $F\left(\boldsymbol{\epsilon}^{*}\right)$, preserves the logical connectives, and satisfies the following conditions similar to Definition $7(\mathrm{~d})$ :

$$
\begin{aligned}
\lceil x \in C\rceil & =C(x), \\
\lceil x=C\rceil=\lceil C=x\rceil & =(\forall y)(\lceil y \in C\rceil \Leftrightarrow y \in x) \\
& =(\forall y)(C(y) \Leftrightarrow y \in x), \\
\lceil C \in x\rceil & =(\exists y)(\lceil y=C\rceil \wedge y \in x) \\
& =(\exists y)((\forall z)(z \in y \Leftrightarrow C(z)) \wedge y \in x), \\
\lceil C=D\rceil & =(\forall y)(\lceil y \in C\rceil \Leftrightarrow\lceil y \in D\rceil) \\
& =(\forall y)(C(y) \Leftrightarrow D(y)), \\
\lceil D(C)\rceil=\lceil C \in D\rceil & =(\exists y)(\lceil y=C\rceil \wedge\lceil y \in D\rceil) \\
& =(\exists y)((\forall z)(z \in y \Leftrightarrow C(z)) \wedge D(y))
\end{aligned}
$$


for each variable $x$ and arbitrary symbols $C, D \in \mathcal{P}$, where $y$ and $z$ are new variables different from $x$. The mapping $\lceil\cdot\rceil$ is called the elimination of external classes.

Let $\mathcal{T}$ be a theory of signature $\epsilon$. The extension of the theory $\mathcal{T}$ by external classes is the (conservative) extension $\mathcal{T}^{*}$ of $\mathcal{T}$ to the above-described signature $\epsilon^{*}$ without any additional special axioms, which is supplemented by the use of the formulas of signature $\epsilon_{\mathrm{c}}^{*}$ and the elimination of external classes as syntactic sugar. As in the case of definable classes (see Definition 7), the signature enrichment $\epsilon^{*} \subset \epsilon_{\mathrm{c}}^{*}$ is not accompanied by any extension of the theory $\mathcal{T}^{*}$ : the axiomatics remain corresponding to the predicate calculus of $\epsilon^{*}$, while the external classes serve as function replacements for the corresponding predicate symbols on the level of grammatics.

As a result, within $\mathcal{T}^{*}$, a sense is given to expressions $\varphi\left(x_{1}, \ldots, x_{m}, C_{1}, \ldots, C_{n}\right)$ obtained from formulas $\varphi$ of set-theoretic signature by replacing some of the parameters with external classes. Moreover, as is easily seen, for each formula $\psi\left(x_{1}, \ldots, x_{m}\right) \in F\left(\boldsymbol{\epsilon}_{\mathrm{c}}^{*}\right)$, there exist a formula $\varphi\left(x_{1}, \ldots, x_{m}, y_{1}, \ldots, y_{n}\right) \in F(\boldsymbol{\epsilon})$ and external classes $C_{1}, \ldots, C_{n} \in \mathcal{P}$ such that

$$
\left\lceil\psi\left(x_{1}, \ldots, x_{m}\right)\right\rceil=\left\lceil\varphi\left(x_{1}, \ldots, x_{m}, C_{1}, \ldots, C_{n}\right)\right\rceil
$$

and, in particular,

$$
\mathcal{T}^{*} \vdash\left(\psi\left(x_{1}, \ldots, x_{m}\right) \Leftrightarrow \varphi\left(x_{1}, \ldots, x_{m}, C_{1}, \ldots, C_{n}\right)\right) .
$$

If external classes are used together with an eliminable extension of a theory and internal classes; the rules (4) are not sufficient for translating the formulas into the language of signature $\epsilon^{*}$. To this end, we should apply, first, an elimination of the extension, next, the elimination of internal classes, and, finally, the elimination of external classes.

Remark 10. The formalism of Definition 21 can be generalized to the case of external classes with parameters. In this case, predicate symbols in $\mathcal{P}$ may have arbitrary nonzero arities, each symbol $C \in \mathcal{P}$ of arity $n+1$ is associated with the symbol of external class having the same name $C$ and arity $n$, and the elimination rules are appropriately specified. For instance, the first rule of (4) takes the form

$$
\begin{aligned}
& \left\lceil x \in C\left(\tau_{1}, \ldots, \tau_{n}\right)\right\rceil \\
& \quad=\left(\exists y_{1}\right) \ldots\left(\exists y_{n}\right)\left(\left\lceil y_{1}=\tau_{1}\right\rceil \wedge \cdots \wedge\left\lceil y_{n}=\tau_{n}\right\rceil \wedge C\left(x, y_{1}, \ldots, y_{n}\right)\right),
\end{aligned}
$$

where $\tau_{1}, \ldots, \tau_{n}$ are terms of signature $\epsilon_{\mathrm{c}}^{*}$. Such a generalization is unnecessary for the present article.

Basing on Definition 21, we will extend the syntax of truth values by the use of Boolean-valued classes.

Definition 22. Let $X$ be a B-system of set-theoretic signature $\epsilon=\{=, \in\}$. Consider some classes $\Phi_{1}, \ldots, \Phi_{n}$ and extend ZFC by the hypothesis $\Phi_{1}, \ldots, \Phi_{n} \Subset X$ (see Proposition 5). Denote by $\epsilon^{*}$ the signature obtained from $\epsilon$ by adding new unary predicate symbols $\operatorname{Sym}\left(\Phi_{1}\right), \ldots, \operatorname{Sym}\left(\Phi_{n}\right)$, and turn $X$ into a B-system of signature $\epsilon^{*}$ by means of the additional interpretations $\left[\operatorname{Sym}\left(\Phi_{1}\right)\right]_{X}=$ $\Phi_{1}, \ldots,\left[\operatorname{Sym}\left(\Phi_{n}\right)\right]_{\mathrm{X}}=\Phi_{n}$. Consider the enrichment $\epsilon_{\mathrm{c}}^{*}$ of $\epsilon^{*}$ by external classes with elimination $\left\lceil\cdot 7: F\left(\boldsymbol{\epsilon}_{\mathrm{c}}^{*}\right) \rightarrow F\left(\boldsymbol{\epsilon}^{*}\right)\right.$ (see Definition 21) and, acting in a similar way to Definition 18, extend the syntax of Boolean truth values by the terms $[\psi]_{X}$ and formulas $X \vDash \psi, \psi \in F\left(\epsilon_{\mathrm{c}}^{*}\right)$, subject to the definitions

$$
[\psi]_{X}=[\lceil\psi\rceil]_{X}, \quad X \vDash \psi \Leftrightarrow[\psi]_{X}=1_{B} .
$$

In order to make the expressions less bulky, we write $\Phi_{i}$ instead of $\operatorname{Sym}\left(\Phi_{i}\right)$ inside $[\ldots]_{X}$. (This informality is easily compensated by the context.) As a result, expressions of the form

$$
\left[\varphi\left(x_{1}, \ldots, x_{m}, \Phi_{1}, \ldots, \Phi_{n}\right)\right]_{X}, \quad X \vDash \varphi\left(x_{1}, \ldots, x_{m}, \Phi_{1}, \ldots, \Phi_{n}\right)
$$

make sense for formulas $\varphi\left(x_{1}, \ldots, x_{m}, y_{1}, \ldots, y_{n}\right)$ of signature $\epsilon$ and classes $\Phi_{i}$ subject to the hypothesis $\Phi_{i} \Subset X$. 
The extended Boolean truth valuation agrees with the logical connectives (cp. (2)) and, according to the rules (4), takes the following values at the new atomic formulas of signature $\epsilon_{\mathrm{c}}^{*}$ :

$$
\begin{aligned}
x_{X}=[x \in \Phi]_{X} & =\Phi(x) ; \\
{[x=\Phi]_{X}=[\Phi=x]_{X} } & =[(\forall y)(y \in \Phi \Leftrightarrow y \in x)]_{X} \\
& =\bigwedge_{y \in X} \Phi(y) \Leftrightarrow_{B}[y \in x]_{X} ; \\
{[\Phi \in x]_{X} } & =[(\exists y)(y=\Phi \wedge y \in x)]_{X} \\
& =\bigvee_{y \in X}\left(\bigwedge_{z \in X}[z \in y]_{X} \Leftrightarrow{ }_{B} \Phi(z)\right) \wedge_{B}[y \in x]_{X} ; \\
{[\Phi=\Psi]_{X} } & =[(\forall x)(x \in \Phi \Leftrightarrow x \in \Psi)]_{X} \\
& =\bigwedge_{x \in X} \Phi(x) \Leftrightarrow{ }_{B} \Psi(x) ; \\
{[\Psi(\Phi)]_{X}=[\Phi \in \Psi]_{X} } & =[(\exists x)(x=\Phi \wedge x \in \Psi)]_{X} \\
& =\bigvee_{x \in X}\left(\bigwedge_{y \in X}[y \in x]_{X} \Leftrightarrow{ }_{B} \Phi(y)\right) \wedge_{B} \Psi(x) .
\end{aligned}
$$

In what follows, when considering Boolean-valued classes in any B-system X of signature $\epsilon$, we will always regard $X$ as a B-system of the corresponding enriched signature $\epsilon^{*}$ and employ the expressions (5).

Under the assumptions of Definition 22, consider a theory $\mathcal{T}$ of signature $\epsilon$ and let $\mathcal{T}^{*}$ be the extension of $\mathcal{T}$ by external classes (see Definition 21). Since $\mathcal{T}^{*}$ extends $\mathcal{T}$ to a richer signature without additional special axioms, $X \vDash \mathcal{T}$ obviously implies $X \vDash \mathcal{T}^{*}$ (with the external classes interpreted as Boolean-valued classes in $X$ ). Therefore, the following strengthened version of Proposition 8 holds:

Proposition 11. Let $\mathcal{T}$ be a theory of signature $\epsilon$; let $\mathcal{T}^{*}$ be the extension of $\mathcal{T}$ by external classes $\Phi_{1}, \ldots, \Phi_{n}$; and let $\Gamma$ and $\Delta$ be some sets of sentences in the language of $\mathcal{T}^{*}$. Extend ZFC by the hypotheses " $X$ is a B-model of $\mathcal{T}$ " and $\Phi_{1}, \ldots, \Phi_{n} \Subset X$.

(a) If $\mathcal{T}^{*} \vdash \Delta$ then $\mathrm{ZFC} \vdash(X \vDash \Delta)$.

(b) If $\mathrm{ZFC} \vdash(X \vDash \Gamma)$ and $\mathcal{T}^{*}, \Gamma \vdash \Delta$ then $\mathrm{ZFC} \vdash(X \vDash \Delta)$.

The following is a consequence of Propositions 7 and 11:

Corollary 1. Let $X$ be a B-system of signature $\epsilon$ and let $\varphi$ be a formula of signature $\epsilon$ with parameters $x_{1}, \ldots, x_{m}, y_{1}, \ldots, y_{n}$. Then, given any Boolean-valued classes $\Phi_{1}, \ldots, \Phi_{n} \Subset X$, the following function is extensional:

$$
\left(x_{1}, \ldots, x_{m}\right) \in X^{m} \mapsto\left[\varphi\left(x_{1}, \ldots, x_{m}, \Phi_{1}, \ldots, \Phi_{n}\right)\right]_{X} \in B .
$$

Remark 11. According to Propositions 8 and 11, given a Boolean-valued model X of a theory $\mathcal{T}$, there is a possibility of proving the validity of a formula in X by "reasoning inside X." Namely, let $\varphi_{1}, \ldots, \varphi_{k}, \varphi$ be formulas with parameters $\dddot{x}=x_{1}, \ldots, x_{m}, \dddot{\Phi}=\Phi_{1}, \ldots, \Phi_{n}$. Assume that, reasoning within $\mathcal{T}$ and treating $\Phi_{i}$ as unary predicates or external classes, we can prove $\varphi$ basing on the hypotheses $\varphi_{1}, \ldots, \varphi_{k}$. Then we may assert that, for arbitrary Boolean-valued classes $\Phi_{1}, \ldots, \Phi_{n} \Subset X$,

$$
X \vDash(\forall \ddot{x})\left(\varphi_{1}(\dddot{x}, \dddot{\Phi}) \wedge \cdots \wedge \varphi_{k}(\ddot{x}, \dddot{\Phi}) \Rightarrow \varphi(\ddot{x}, \dddot{\Phi})\right) .
$$

In particular, for all $\dddot{x} \in X$, the validity $X \vDash \varphi_{1}(\dddot{x}, \ddot{\Phi}), \ldots, X \vDash \varphi_{k}(\ddot{x}, \ddot{\Phi})$ implies $X \vDash \varphi(\dddot{x}, \ddot{\Phi})$. 


\section{Basic Technique}

The main tools in dealing with Boolean-valued systems include the apparatus of partial elements, joins of antichains, mixings of subclasses, ascents and descents of various kinds, as well as the use of Boolean-valued classes in the language of truth values. Another useful tool is the analog of Lévy's Lemma on the absoluteness of bounded formulas for transitive Boolean-valued subsystems. In this section, we also introduce and study intensional, predicative, cyclic, regular, and $\sigma$-regular Boolean-valued systems, examine the maximum principle, and analyze its relationship with the ascent and mixing principles.

\subsection{Partial Elements}

Partial elements of a Boolean-valued system are abstract analogs of partially defined functions: the part $\left.x\right|_{b}$ of an element $x$ with domain $b$ resembles the restriction of an everywhere defined function $x$ onto a subset $b$. In this subsection, we introduce and develop the technique of partial elements and present formalization for using partial elements in the language of truth values.

Let $X$ be a $B$-system of an arbitrary predicative signature $\Sigma$ with equality.

Definition 23. Introduce the equivalence $\sim$ on the class $X \times B$ as follows:

$$
(x, b) \sim(y, c) \Leftrightarrow b=c \wedge[x=y]_{X} \geqslant_{B} b .
$$

Define the quotient $\% X:=(X \times B) / \sim$ by using the so-called Frege-Russell-Scott trick (see [4] (1.5.8), [5] (1.6.8)):

$$
\begin{aligned}
& { }^{\%} X:=\{\sim(x, b):(x, b) \in X \times B\} ; \\
& \sim(x, b):=\left\{(y, b): y \in X,[x=y]_{X} \geqslant_{B} b,\right. \\
& \left.(\forall z \in X)\left([x=z]_{X} \geqslant_{B} b \Rightarrow \operatorname{rank}(y) \leqslant \operatorname{rank}(z)\right)\right\},
\end{aligned}
$$

where $\operatorname{rank}(y)$ is the rank of a set $y$ in the von Neumann cumulative hierarchy. By this approach, the cosets $\sim(x, b)$ corresponding to pairs $(x, b) \in X \times B$ occur to be sets even in the case of a proper class $X$. Denote the coset $\sim(x, b)$ by $\left.x\right|_{b}$ and call it a partial element of $X$ or, more exactly, the part of $x$ with domain $b$ or the restriction of $x$ onto $b$. The domain $b$ of a partial element $p=\left.x\right|_{b}$ is denoted by $\Delta p$. Given $p=\left.x\right|_{b}$ and $c \in B$, put $\left.p\right|_{c}:=\left.x\right|_{b \wedge c}$. Moreover, granted $P \subset X \cup{ }^{\%} X$ and $c \in B$, introduce the notation

$$
\left.P\right|_{c}:=\left\{\left.p\right|_{c}: p \in P\right\}, \quad{ }^{\%} P:=\left\{\left.p\right|_{b}: p \in P, b \in B\right\}=\left.\bigcup_{b \in B} P\right|_{b} .
$$

If $p \in{ }^{\%} X$ and $\bar{p} \in X \cup{ }^{\%} X$; then write $p \sqsubset \bar{p}$, say that $p$ is a part or restriction of $\bar{p}$, and call $\bar{p}$ an extension of $p$, whenever $(\exists b \in B) p=\left.\bar{p}\right|_{b}$ or, which is the same, $p=\left.\bar{p}\right|_{\Delta p}$.

Definition 24. A partial element $p \in{ }^{\%} X$ is called everywhere defined or global if $\Delta p=1_{B}$ or, which is the same, $p=\left.x\right|_{1_{B}}$ for some $x \in X$. As is easily seen,

$$
\left.x\right|_{1_{B}}=\left.y\right|_{1_{B}} \Leftrightarrow[x=y]_{X}=1_{B} \Leftrightarrow x \sim y
$$

for all $x, y \in X$. In the sequel, we denote $\left.x\right|_{1_{B}}$ by $\tilde{x}$ and write the relation $\tilde{x}=p$ as $x \sim p$ or $p \sim x$. Moreover, given $Y \subset X$, put

$$
\widetilde{Y}:=\{\widetilde{y}: y \in Y\}=\left.Y\right|_{1_{B}} .
$$

If $X$ is separated then the equalities $\widetilde{x}=\widetilde{y}$ and $x=y$ are equivalent. In this case, we identify the elements $x \in X$ with the corresponding global partial elements $\widetilde{x} \in \widetilde{X}$ and thus assume that $X \subset{ }^{\%} X$.

Propose an agreement on using partial elements in the language of Boolean truth values. 
Definition 25. Consider the signature $\Sigma^{\%}$ obtained from $\Sigma$ by adding the constant $\% p$ for each set-theoretic term $p$, and introduce the terms $[\psi]_{X}, \psi \in F\left(\Sigma^{\%}\right)$, as follows (cp. Definition 19): Given a formula $\varphi(\ddot{x}, \ddot{y})$ of signature $\Sigma$ whose parameters are arbitrarily partitioned into two parts, $\ddot{x}=x_{1}, \ldots, x_{m}$ and $\ddot{y}=y_{1}, \ldots, y_{n}$, and given arbitrary set-theoretic terms $\ddot{p}=p_{1}, \ldots, p_{n}$; consider the parameters $\ddot{z}$ of the set $\ddot{x} \cup \dddot{p}$ (see Definition 2); enrich the language of ZFC by the function symbol $\langle\varphi(\ddot{x}, \% \dddot{p})\rangle_{X}$ of arity $|\ddot{z}|$, with $\% \dddot{p}=\% p_{1}, \ldots, \% p_{n}$; introduce the notation

$$
[\varphi(\dddot{x}, \% \dddot{p})]_{X}:=\langle\varphi(\dddot{x}, \% \dddot{p})\rangle_{X}(\dddot{z}) ;
$$

and extend ZFC by the defining axiom

$$
\begin{aligned}
b=\left[\varphi\left(\ddot{x}, \% p_{1}, \ldots, \% p_{n}\right)\right]_{X} \Leftrightarrow\left(\exists y_{1}, \ldots, y_{n} \in X\right)\left(\exists b_{1}, \ldots, b_{n} \in B\right) \\
\\
p_{1}=\left.y_{1}\right|_{b_{1}} \wedge \cdots \wedge p_{n}=\left.y_{n}\right|_{b_{n}} \wedge \\
b=\left[\varphi\left(\ddot{x}, y_{1}, \ldots, y_{n}\right)\right]_{X} \wedge_{B} b_{1} \wedge_{B} \cdots \wedge_{B} b_{n}
\end{aligned}
$$

subject to the conditions $\ddot{x} \in X, p_{1}, \ldots, p_{n} \in{ }^{\%} X$. Agree to write $p$ instead of $\% p$ inside $[\ldots]_{X}$. (The formal syntax can be easily restored from the context.) Therefore, the above definition rewrites to the equality

$$
\left[\varphi\left(\dddot{x}, p_{1}, \ldots, p_{n}\right)\right]_{X}=\left[\varphi\left(\dddot{x}, y_{1}, \ldots, y_{n}\right)\right]_{X} \wedge_{B} \Delta p_{1} \wedge_{B} \cdots \wedge_{B} \Delta p_{n}, \quad p_{i}=\left.y_{i}\right|_{\Delta p_{i}} .
$$

In the case of $\Sigma=\{=, \in\}$ we also extend the truth valuation to the formulas that contain both partial elements and Boolean-valued classes $\Phi, \ddot{\Phi} \Subset X$ in exactly the same manner:

$$
\begin{aligned}
& {\left[\varphi\left(\ddot{x}, p_{1}, \ldots, p_{n}, \dddot{\Phi}\right)\right]_{X}=\left[\varphi\left(\dddot{x}, y_{1}, \ldots, y_{n}, \dddot{\Phi}\right)\right]_{X} \wedge_{B} \Delta p_{1} \wedge_{B} \cdots \wedge_{B} \Delta p_{n}, \quad p_{i}=\left.y_{i}\right|_{\Delta p_{i}} ;} \\
& {[\Phi(p)]_{X}=\Phi(y) \wedge_{B} \Delta p, \quad p=\left.y\right|_{\Delta p} .}
\end{aligned}
$$

The definitions are correct (see Agreement 4), since the right-hand sides of the equalities (7) and (8) do not depend on the choice of representatives $\left(y_{i}, \Delta p_{i}\right)$ of the cosets $p_{i}$. Indeed, if $\left.y_{i}\right|_{\Delta p_{i}}=\left.z_{i}\right|_{\Delta p_{i}}$ then $\left[y_{i}=z_{i}\right]_{X} \geqslant_{B} \Delta p_{i}$, and, by Proposition 7 and Corollary 1, we have

$$
\begin{aligned}
& {\left[\varphi\left(\ddot{x}, y_{1}, \ldots, y_{n}, \ldots\right)\right]_{X} \wedge_{B} \Delta p_{1} \wedge_{B} \cdots \wedge_{B} \Delta p_{n}} \\
& \quad=\left[\varphi\left(\ddot{x}, z_{1}, \ldots, z_{n}, \ldots\right)\right]_{X} \wedge_{B} \Delta p_{1} \wedge_{B} \cdots \wedge_{B} \Delta p_{n} .
\end{aligned}
$$

Observe that the above semantics of "partially defined terms" does not correspond to any form of free logic, and the truth valuation on $F\left(\Sigma^{\%}\right)$ does not agree with negation: for instance, if $p \in{ }^{\%} \mathrm{X}$ and $\Delta p=0_{B}$ then $[p=p]_{X}=[\neg(p=p)]_{X}=0_{B}$.

With Definition 25 taken into account, the statements of Proposition 7 and Corollary 1 extend to the case of partial elements:

Proposition 12. Let $X$ be a B-system of signature $\Sigma$ and let $\varphi$ be a formula of signature $\Sigma$ that has parameters $x_{1}, \ldots, x_{n}$ and optionally contains occurrences of partial elements of $X$ and, in the case of $\Sigma=\{=, \in\}$, Boolean-valued classes in X. Then the following function is extensional:

$$
\left(x_{1}, \ldots, x_{n}\right) \in X^{n} \mapsto\left[\varphi\left(x_{1}, \ldots, x_{n}\right)\right]_{X} \in B .
$$

In particular, for all $p, q \in{ }^{\%} X$,

$$
[\varphi(p, \ldots)]_{X} \wedge_{B}[p=q]_{X}=[\varphi(q, \ldots)]_{X} \wedge_{B}[p=q]_{X} .
$$

Remark 12. Due to Proposition 12, we may substantially simplify the statements of the general assertions on the truth values of formulas: Instead of considering such expressions as $[\varphi(x, \ddot{y}, \ddot{p}, \ddot{\Phi})]_{X}$ or $[(\exists x) \varphi(x, \dddot{y}, \ddot{p}, \ddot{\Phi})]_{X}$ for an arbitrary formula $\varphi(x, \ddot{y}, \ddot{u}, \ddot{v})$, elements $x, \ddot{y} \in X$, partial elements 
$\ddot{p} \in{ }^{\%} X$, and Boolean-valued classes $\ddot{\Phi} \Subset X$, it suffices to speak of the values $\Phi(x)$ or $[(\exists x) \Phi(x)]_{X}$ for some class $\Phi \Subset X$ (see, e.g., Lemmas 6 and 16 and Corollary 2).

\subsection{Ascents and Intensionality}

Ascents are the key tool in dealing with Boolean-valued systems of set-theoretic signature. Given a $B$-valued system $X$, we introduce and study the ascents of three types: the ascents $P \uparrow$ of subclasses $P \subset{ }^{\%} X$ of partial elements, the ascents $Y \uparrow$ of subclasses $Y \subset X$ of elements, and the ascents $\Phi \uparrow$ of Boolean-valued functions $\Phi: Y \rightarrow B$. In all the cases, the ascents are Boolean-valued classes and therefore can be used in the language of Boolean truth values (see Definition 22). Another basic notion considered in this subsection is representation of Boolean-valued classes by elements of the system. The system $X$ is called intensional if the ascents $P \uparrow$ of all sets $P \subset{ }^{\%} X$ are represented in $X$. This is one of the main conditions in the axiomatic characterization of Boolean-valued universe; see [1] (3.4(4)).

The rest of the paper is devoted to the study of Boolean-valued algebraic systems of set-theoretic signature $\{=, \in\}$. Therefore, by a formula we will always mean a formula of signature $\{=, \in\}$ (or of a richer signature obtained by formal definitions), and by a B-system, a $B$-system of signature $\{=, \in\}$. To make expressions less bulky, we will usually omit the indices $B$ and $X$ in the symbols $\wedge_{B},[\ldots]_{X}$, and so forth.

Definition 26. In what follows, $B$ is a complete Boolean algebra and $X$ is a B-system. According to Definitions 10 and 13 and Proposition 6, the latter means that $X,[=]_{X}$, and $[\in]_{X}$ are classes subject to the conjunction of the following formulas:

$$
\begin{aligned}
& X \neq \varnothing, \quad[=]_{X}: X^{2} \rightarrow B, \quad[\in]_{X}: X^{2} \rightarrow B, \\
& \text { for all } x, y, z \in X, \\
& \qquad[=]_{X}(x, x)=1_{B}, \quad[=]_{X}(x, y)=[=]_{X}(y, x), \quad[=]_{X}(x, y) \wedge[=]_{X}(y, z) \leqslant[=]_{X}(x, z), \\
& \quad[\in]_{X}(x, y) \wedge[=]_{X}(y, z) \leqslant[\in]_{X}(x, z), \quad[\in]_{X}(x, y) \wedge[=]_{X}(x, z) \leqslant[\in]_{X}(z, y) .
\end{aligned}
$$

Definition 27. Recall that the equivalence $\sim$ is defined on $X$ by the rule

$$
x \sim y \Leftrightarrow[x=y]=1_{B} \Leftrightarrow X \vDash(x=y),
$$

and the system is called separated whenever $x \sim y \Leftrightarrow x=y$ for all $x, y \in X$ (see Definition 15).

Given an arbitrary element $x \in X$, define the function $[\cdot \in x]: X \rightarrow B$ by putting $[\cdot \in x](z)=$ $[z \in x]$ for all $z \in X$. Consider the following equivalence $\simeq$ on $X:$

$$
x \simeq y \Leftrightarrow[\cdot \in x]=[\cdot \in y] \Leftrightarrow X \vDash(\forall z)(z \in x \Leftrightarrow z \in y) .
$$

As is easily seen, $x \sim y \Rightarrow x \simeq y$. Say that $X$ is extensional whenever $x \sim y \Leftrightarrow x \simeq y$ for all $x, y \in X$ or, which is the same, if the axiom of extensionality is valid in $X:$

$$
X \vDash(\forall x, y)((\forall z)(z \in x \Leftrightarrow z \in y) \Rightarrow x=y) .
$$

A separated extensional system is characterized by the fact that its elements are uniquely determined by the truth values of the containment: $x=y \Leftrightarrow(\forall z \in X)[z \in x]=[z \in y]$.

Definition 28. The ascent of a set or class $P \subset{ }^{\%} X$ is the function (a class function if $X$ is a proper class) defined as follows:

$$
P \uparrow: X \rightarrow B, \quad P \uparrow(x)=\bigvee_{p \in P}[x=p] .
$$


Given $Y \subset X$ and $\Psi: Y \rightarrow B$, put $Y \uparrow:=\widetilde{Y} \uparrow$ and $\Psi \uparrow:=\left\{\left.y\right|_{\Psi(y)}: y \in Y\right\} \uparrow$, that is,

$$
\begin{aligned}
& Y \uparrow: X \rightarrow B, \quad Y \uparrow(x)=\bigvee_{y \in Y}[x=y], \\
& \Psi \uparrow: X \rightarrow B, \quad \Psi \uparrow(x)=\bigvee_{y \in Y}[x=y] \wedge \Psi(y) .
\end{aligned}
$$

Theorem 2. The following properties of a function $\Phi: X \rightarrow B$ are equivalent:

(a) $\Phi \Subset X$, that is, $\Phi$ is extensional (see Proposition 5);

(b) $\Phi=P \uparrow$ for some set or class $P \subset{ }^{\%} X$;

(c) $\Phi=\left\{\left.x\right|_{\Phi(x)}: x \in X\right\} \uparrow$;

(d) $\Phi=\Psi \uparrow$, where $\Psi: Y \rightarrow B$ and $Y \subset X$ is a set or class;

(e) $\Phi=\Phi \uparrow$

Proof. (a) $\Rightarrow$ (c): If $y \in X$ then

$$
\left\{\left.x\right|_{\Phi(x)}: x \in X\right\} \uparrow(y)=\bigvee_{x \in X}\left[y=\left.x\right|_{\Phi(x)}\right]=\bigvee_{x \in X}[y=x] \wedge \Phi(x)=\bigvee_{x \in X}[y=x] \wedge \Phi(y)=\Phi(y)
$$

The implications $(\mathrm{c}) \Rightarrow(\mathrm{e}) \Rightarrow(\mathrm{d}) \Rightarrow(\mathrm{b})$ are trivial.

(b) $\Rightarrow(a):$ If $x, y \in X$ then

$$
P \uparrow(x) \wedge[x=y]=\bigvee_{p \in P}[x=p] \wedge[x=y] \leqslant \bigvee_{p \in P}[y=p]=P \uparrow(y) .
$$

According to Theorem 2, the ascents $P \uparrow$ are Boolean-valued classes, which fact allows us to use them in the language of Boolean truth values (see Definition 22). Therefore, the expressions of the form $\left[\varphi\left(x_{1}, \ldots, x_{m}, p_{1}, \ldots, p_{n}, P_{1} \uparrow, \ldots, P_{k} \uparrow\right)\right]$ make sense, where $\varphi$ is a formula, $x_{1}, \ldots, x_{m} \in X, p_{1}, \ldots, p_{n} \in{ }^{\%} X$, and $P_{1}, \ldots, P_{k} \subset{ }^{\%} X$ (see Definition 25). The following lemma lists several useful equalities that employ such expressions.

Lemma 2. Let $q \in{ }^{\%} X, P \subset{ }^{\%} X$, and $Y \subset X$. Then

(a) $[q \in P \uparrow]=\bigvee_{p \in P}[q=p]$;

(b) $[P \uparrow \neq \varnothing]=\bigvee_{x \in X} P \uparrow(x)=\bigvee_{p \in P} \Delta p$;

(c) $P \subset{ }^{\%} Y \Rightarrow[P \uparrow \neq \varnothing]=\bigvee_{y \in Y} P \uparrow(y)$.

Proof. (a): If $x \in X$ and $b \in B$ then

$$
\left[\left.x\right|_{b} \in P \uparrow\right]=[x \in P \uparrow] \wedge b=P \uparrow(x) \wedge b=\bigvee_{p \in P}[x=p] \wedge b=\bigvee_{p \in P}\left[\left.x\right|_{b}=p\right] .
$$

(b): If $P=\left\{\left.x_{i}\right|_{b_{i}}: i \in I\right\}$, with $x_{i} \in X$ and $b_{i} \in B$, then

$$
\begin{gathered}
{[P \uparrow \neq \varnothing]=[(\exists x)(x \in P \uparrow)]=\bigvee_{x \in X}[x \in P \uparrow]=\bigvee_{x \in X} P \uparrow(x)} \\
=\bigvee_{x \in X} \bigvee_{i \in I}\left[x=\left.x_{i}\right|_{b_{i}}\right]=\bigvee_{i \in I} \bigvee_{x \in X}\left[x=x_{i}\right] \wedge b_{i}=\bigvee_{i \in I} b_{i} .
\end{gathered}
$$

(c): If $P=\left\{\left.y_{i}\right|_{b_{i}}: i \in I\right\}$, with $y_{i} \in Y$ and $b_{i} \in B$, then

$$
\begin{aligned}
{[P \uparrow \neq \varnothing] } & =\bigvee_{i \in I} \bigvee_{x \in X}\left[x=y_{i}\right] \wedge b_{i}=\bigvee_{i \in I} \bigvee_{y \in Y}\left[y=y_{i}\right] \wedge b_{i} \\
& =\bigvee_{y \in Y} \bigvee_{i \in I}\left[y=\left.y_{i}\right|_{b_{i}}\right]=\bigvee_{y \in Y} P \uparrow(y) .
\end{aligned}
$$


Given functions $\Phi, \Psi: Y \rightarrow B$ on a subclass $Y \subset X$, write $\Phi \leqslant \Psi$ whenever $\Phi(y) \leqslant \Psi(y)$ for all $y \in Y$.

Lemma 3. Consider $Y \subset X$ and $\Psi: Y \rightarrow B$. The function $\Psi \uparrow: X \rightarrow B$ is the least extensional dominant of $\Psi$ :

(a) $\Psi \leqslant\left.(\Psi \uparrow)\right|_{Y}$;

(b) if $\Psi: X \rightarrow B$ is extensional and $\Psi \leqslant\left.\bar{\Psi}\right|_{Y}$ then $\Psi \uparrow \leqslant \bar{\Psi}$.

Proof. (b): If $\bar{\Psi}: X \rightarrow B$ is extensional and $(\forall y \in Y) \Psi(y) \leqslant \bar{\Psi}(y)$ then, for all $x \in X$,

$$
\Psi \uparrow(x)=\bigvee_{y \in Y}[x=y] \wedge \Psi(y) \leqslant \bigvee_{y \in Y}[x=y] \wedge \bar{\Psi}(y)=\bar{\Psi}(x)=\bar{\Psi}(x)
$$

Lemma 4. If $\Phi \Subset X$ and $P \subset{ }^{\%} X$ then $[\Phi \in P \uparrow]=\bigvee_{p \in P}[\Phi=p]$.

Proof. Let $P=\left\{\left.x_{i}\right|_{b_{i}}: i \in I\right\}$, with $x_{i} \in X$ and $b_{i} \in B$. According to the rules (6) we have

$$
\begin{gathered}
{[\Phi \in P \uparrow]=[(\exists x)(\Phi=x \wedge x \in P \uparrow)]=\bigvee_{x \in X}[\Phi=x] \wedge \bigvee_{i \in I}\left[x=x_{i}\right] \wedge b_{i}} \\
=\bigvee_{i \in I} \bigvee_{x \in X}[\Phi=x] \wedge\left[x=x_{i}\right] \wedge b_{i}=\bigvee_{i \in I}\left[\Phi=x_{i}\right] \wedge b_{i}=\bigvee_{p \in P}[\Phi=p]
\end{gathered}
$$

Lemma 5.

(a) If $\Phi, \Psi \Subset X$ then $X \vDash(\Phi \subset \Psi) \Leftrightarrow \Phi \leqslant \Psi$.

(b) If $Y \subset X$ and $P \subset{ }^{\%} Y$ then $X \vDash(P \uparrow \subset Y \uparrow)$.

(c) If $Y \subset X, \Phi \Subset X$, and $P=\left\{\left.y\right|_{\Phi(y)}: y \in Y\right\}$ then $X \vDash(P \uparrow=Y \uparrow \cap \Phi)$ and $[\Phi \subset Y \uparrow]=$ $[\Phi=P \uparrow]$. In particular, if $X \vDash(\Phi \subset Y \uparrow)$ then $X \vDash(\Phi=P \uparrow)$ for some subclass $P \subset{ }^{\%} Y$.

(d) If $Z \subset Y \subset X$ then $X \vDash(Y \uparrow \backslash Z \uparrow \subset(Y \backslash Z) \uparrow)$.

Proof. Assertion (a) is obvious; (b) follows from (a).

(c): For all $x \in X$,

$$
[x \in P \uparrow]=\bigvee_{y \in Y}[x=y] \wedge \Phi(y)=\bigvee_{y \in Y}[x=y] \wedge \Phi(x)=[x \in Y \uparrow \cap \Phi],
$$

that is, $X \vDash(P \uparrow=Y \uparrow \cap \Phi)$ and so $[\Phi \subset Y \uparrow]=[\Phi=Y \uparrow \cap \Phi]=[\Phi=P \uparrow]$.

(d): For all $x \in X$,

$$
\begin{aligned}
{[x \in Y \uparrow \backslash Z \uparrow] } & =\bigvee_{y \in Y}[x=y] \wedge[x \notin Z \uparrow] \\
& =\left(\bigvee_{y \in Y \backslash Z}[x=y] \wedge[x \notin Z \uparrow]\right) \vee\left(\bigvee_{y \in Z}[x=y] \wedge[x \notin Z \uparrow]\right) \\
& \leqslant\left(\bigvee_{y \in Y \backslash Z}[x=y]\right) \vee([x \in Z \uparrow] \wedge[x \notin Z \uparrow])=[x \in(Y \backslash Z) \uparrow]
\end{aligned}
$$

Lemma 6. Let $P \subset{ }^{\%} X$ and $\Phi \Subset X$. Then
(a) $[(\exists x \in P \uparrow) \Phi(x)]=\bigvee_{p \in P}[\Phi(p)]$;
(b) $[(\forall x \in P \uparrow) \Phi(x)]=\Lambda_{p \in P}[\Phi(p)] \vee \neg \Delta p$;
(c) $X \vDash(\forall x \in P \uparrow) \Phi(x) \Leftrightarrow(\forall p \in P)[\Phi(p)]=\Delta p$. 
Proof. (a): With the equality $[\Phi(x)] \wedge[x=p]=[\Phi(p)] \wedge[x=p]$ taken into account, we have

$$
\begin{aligned}
{[(\exists x \in P \uparrow) \Phi(x)] } & =\bigvee_{x \in X}[\Phi(x)] \wedge[x \in P \uparrow] \\
& =\bigvee_{x \in X} \bigvee_{p \in P}[\Phi(x)] \wedge[x=p]=\bigvee_{x \in X} \bigvee_{p \in P}[\Phi(p)] \wedge[x=p] \\
& =\bigvee_{p \in P}[\Phi(p)] \wedge \bigvee_{x \in X}[x=p]=\bigvee_{p \in P}[\Phi(p)] \wedge \Delta p=\bigvee_{p \in P}[\Phi(p)] .
\end{aligned}
$$

The relation (b) is easily deduced from (a); (c) is a partial case of (b).

Corollary 2. Let $Y \subset X$ and $\Phi \Subset X$. Then

(a) $[(\exists y \in Y \uparrow) \Phi(y)]=\bigvee_{y \in Y}[\Phi(y)]$;

(b) $[(\forall y \in Y \uparrow) \Phi(y)]=\bigwedge_{y \in Y}[\Phi(y)]$;

(c) $X \vDash(\forall y \in Y \uparrow) \Phi(y) \Leftrightarrow(\forall y \in Y) X \vDash \Phi(y)$.

Definition 29. Say that an element $x \in X$ represents a Boolean-valued class $\Phi \Subset X$ and write $x \simeq \Phi$ or $\Phi \simeq x$, if $[\cdot \in x]=\Phi$ (see Definition 27). Therefore,

$$
x \simeq \Phi \Leftrightarrow(\forall y \in X)[y \in x]=\Phi(y) \Leftrightarrow X \vDash(x=\Phi) .
$$

As is easily seen, for all $x \in X$, the function $[\cdot \in x]: X \rightarrow B$ is extensional; and so every element $x \in X$ represents the Boolean-valued class $[\cdot \in x]$. An element $x \in X$ represents the ascent $P \uparrow$ of $a$ set or class $P \subset{ }^{\%} X$ if $x \simeq P \uparrow$; that is,

$$
(\forall y \in X)[y \in x]=[y \in P \uparrow]=\bigvee_{p \in P}[y=p] .
$$

In particular, if $x_{i} \in X$ and $b_{i} \in B(i \in I)$ then

$$
x \simeq\left\{\left.x_{i}\right|_{b_{i}}: i \in I\right\} \uparrow \Leftrightarrow(\forall y \in X)[y \in x]=\bigvee_{i \in I}\left[y=x_{i}\right] \wedge b_{i} .
$$

Given an arbitrary subclass $Y \subset X$, denote by $\mathcal{P}_{X}(Y)$ the totality of all elements that represent the ascents of subsets of $\%$ :

$$
\mathcal{P}_{X}(Y):=\left\{x \in X:\left(\exists P \subset{ }^{\%} Y\right)(x \simeq P \uparrow)\right\} .
$$

Call a Boolean-valued system X intensional if the ascents of arbitrary sets of partial elements are represented in $X$ :

$$
\left(\forall P \subset{ }^{\%} X\right)(\exists x \in X)(x \simeq P \uparrow) .
$$

Remark 13. An element $x \in X$ representing a Boolean-valued class $\Phi \Subset X$ is uniquely determined up to the equivalence $\simeq$ and, if $X$ is extensional, up to the equivalence $\sim$. In the latter case, we will identify the Boolean-valued class $\Phi$ with the corresponding coset $\widetilde{x}$. Therefore, if $X$ is extensional and $x \simeq \Phi$ then $x \sim \Phi \in \widetilde{X}$. If $X$ is extensional and separated, then the agreement of Definition 24 takes effect, the representable Boolean-valued classes become elements of $X$, and the relation $x \simeq \Phi$ turns into the equality $x=\Phi$.

Definition 30. Given B-systems $X, Y$ and a correspondence $f \subset X \times Y$, define $f{ }^{\%} \subset{ }^{\%} X \times{ }^{\%} Y$ as follows:

$$
f^{\%}:=\left\{\left(\left.x\right|_{b},\left.y\right|_{b}\right):(x, y) \in f, b \in B\right\} .
$$

If $f: X \rightarrow Y$ then $f^{\%}:{ }^{\%} X \rightarrow{ }^{\%} Y$ and $\Delta p=\Delta f^{\%}(p)$ for all $p \in{ }^{\%} X$.

The next assertion is easily proven by induction on the complexity of a formula. 
Proposition 13. Let $\varphi$ be an arbitrary formula. If $f$ is an isomorphism between B-systems $X$ and $Y$; then $f^{\%}$ is a bijection between $\%$ and $\%$, and

$$
\begin{aligned}
& \quad\left[\varphi\left(x_{1}, \ldots, x_{m}, p_{1}, \ldots, p_{n}, P_{1} \uparrow, \ldots, P_{k} \uparrow\right)\right]_{X} \\
& \quad=\left[\varphi\left(f\left(x_{1}\right), \ldots, f\left(x_{m}\right), f^{\%}\left(p_{1}\right), \ldots, f^{\%}\left(p_{n}\right), f^{\%}\left(P_{1}\right) \uparrow, \ldots, f^{\%}\left(P_{k}\right) \uparrow\right)\right]_{Y}
\end{aligned}
$$

for all $x_{1}, \ldots, x_{m} \in X, p_{1}, \ldots, p_{n} \in{ }^{\%} X$, and $P_{1}, \ldots, P_{k} \subset{ }^{\%} X$.

\subsection{Saturated Descents and Predicativity}

Let $B$ be a complete Boolean algebra and let $X$ be a $B$-system. (Recall that the signature is assumed to be set-theoretic.)

In this subsection, we introduce and study the notion of saturated descent $\Phi \Downarrow \subset{ }^{\%} X$ of a Boolean-valued class $\Phi \Subset X$. The class $\Phi \Downarrow$ occurs to be the largest class of partial elements whose ascent equals $\Phi$. A Boolean-valued class $\Phi$ is called predicative if it is the ascent of a set or, which is equivalent, if $\Phi \Downarrow$ is a set. The system $X$ is called predicative whenever all elements of $X$ represent predicative classes. Predicativity is the last of the main conditions in the axiomatic characterization of Boolean-valued universe; see [1] (3.4(5)).

Definition 31. Define the saturated descent $\Phi \Downarrow$ of a Boolean-valued class $\Phi \Subset X$ as follows:

$$
\Phi \Downarrow:=\left\{p \in{ }^{\%} X:[p \in \Phi]=\Delta p\right\}=\left\{\left.x\right|_{b}: x \in X, b \leqslant \Phi(x)\right\} .
$$

The saturated descent $x \Downarrow$ of an element $x \in X$ is defined as the saturated descent $[\cdot \in x] \Downarrow$ of the Boolean-valued class $[\cdot \in x]$ (see Definition 27). Observe that $\Phi \Downarrow$ and $x \Downarrow$ can be proper classes.

Definition 32. Call a class $P \subset{ }^{\%} X$ saturated if $P$ satisfies the two conditions:

$$
\begin{array}{ll}
\left.p \in P \Rightarrow p\right|_{b} \in P, & p \in{ }^{*} X, b \in B ; \\
\left.(\forall a \in A)\left(\left.x\right|_{a} \in P\right) \Rightarrow x\right|_{\vee A} \in P, & x \in X, A \subset B .
\end{array}
$$

Theorem 3. The following properties of a class $P \subset{ }^{\%} X$ are equivalent:

(a) $P$ is saturated;

(b) $(\forall x \in X)(\exists c \in B)(\forall b \in B)\left(\left.x\right|_{b} \in P \Leftrightarrow b \leqslant c\right)$;

(c) $(\forall x \in X)(\forall b \in B)\left(b \leqslant\left. P \uparrow(x) \Rightarrow x\right|_{b} \in P\right)$;

(d) $\left(\forall q \in{ }^{\%} X\right)([q \in P \uparrow]=\Delta q \Rightarrow q \in P)$;

(e) given a subclass $Q \subset{ }^{\%} X$, the equality $Q \uparrow=P \uparrow$ implies $Q \subset P$;

(f) $P=P \uparrow \Downarrow$;

(g) $P=\Phi \Downarrow$ for some Boolean-valued class $\Phi \Subset X$.

Proof. (a) $\Rightarrow$ (b): Given $x \in X$, put $c=\vee A$, with $A=\left\{a \in B:\left.x\right|_{a} \in P\right\}$. From (10) we have $\left.x\right|_{c} \in P$. The rest follows from (9).

(b) $\Rightarrow$ (c): Given $x \in X$ and $b \in B$ with $b \leqslant P \uparrow(x)$, put $A=\left\{a \in B:\left.x\right|_{a} \in P\right\}$. By (b) there is $c \in B$ such that $(\forall a \in B)(a \in A \Leftrightarrow a \leqslant c)$. Then $b \leqslant P \uparrow(x)=\bigvee_{p \in P}[x=p] \leqslant$ $\vee_{a \in A}\left[x=\left.x\right|_{a}\right]=\vee A \leqslant c$ and hence $b \in A$.

(c) $\Rightarrow(\mathrm{d})$ : If $x \in X, b \in B$, and $\left[\left.x\right|_{b} \in P \uparrow\right]=b$, then $b=[x \in P \uparrow] \wedge b \leqslant[x \in P \uparrow]=P \uparrow(x)$ and so $\left.x\right|_{b} \in P$ by (c).

(d) $\Rightarrow(\mathrm{e})$ : If $Q \subset{ }^{\%} X, Q \uparrow=P \uparrow$, and $q \in Q$, then $[q \in P \uparrow]=[q \in Q \uparrow]=\Delta q$ and hence $q \in P$ by $(\mathrm{d})$.

(e) $\Rightarrow(\mathrm{f})$ : By Definition 31, $P \uparrow \Downarrow=Q$, with $Q=\left\{q \in{ }^{\%} X:[q \in P \uparrow]=\Delta q\right\}$. It is clear that $P \subset Q$ and so $P \uparrow \leqslant Q \uparrow$. On the other hand, for each $x \in X$ we have $Q \uparrow(x)=$ $\bigvee_{q \in Q}[x=q]=\bigvee_{q \in Q}[x=q] \wedge \Delta q=\bigvee_{q \in Q}[x=q] \wedge[q \in P \uparrow] \leqslant[x \in P \uparrow]=P \uparrow(x)$. Therefore, $Q \uparrow=P \uparrow$ and hence $Q=P$ due to (e).

The implication $(\mathrm{f}) \Rightarrow(\mathrm{g})$ is trivial.

$(\mathrm{g}) \Rightarrow(\mathrm{a})$ : Owing to $(\mathrm{g})$ and Definition 31 we have $P=\left\{\left.x\right|_{b}: x \in X, b \leqslant \Phi(x)\right\}$ for some $\Phi \Subset X$, whence (9) and (10) are easily verified. 
By Theorem 2, a function $\Phi: X \rightarrow B$ is extensional if and only if $\Phi=P \uparrow$ for some class $P \subset{ }^{\%} X$. The following assertion states that the saturated descent $\Phi \Downarrow$ plays the role of such a $P$.

Lemma 7. If $\Phi \Subset X$ then $\Phi=\Phi \Downarrow \uparrow$. In particular, $P \uparrow \Downarrow \uparrow=P \uparrow$ and $x \simeq x \Downarrow \uparrow$ for $P \subset{ }^{\%} X, x \in X$.

Proof. Given $x \in X$ and using the containment $\left.x\right|_{\Phi(x)} \in \Phi \Downarrow$, we conclude that

$$
\Phi(x)=[x=x] \wedge \Phi(x)=\left[x=\left.x\right|_{\Phi(x)}\right] \leqslant \bigvee_{p \in \Phi \Downarrow}[x=p]
$$

On the other hand, due to extensionality of $\Phi$, for all $y \in X$ and $b \leqslant \Phi(y)$, we have

$$
\left[x=\left.y\right|_{b}\right]=[x=y] \wedge b \leqslant[x=y] \wedge \Phi(y) \leqslant \Phi(x) .
$$

The next assertion is a consequence of Theorem 3 and Lemma 7.

Corollary 3. Let $\Phi \Subset X$ and $P \subset{ }^{\%} X$. The following are equivalent:

(a) $P$ is saturated and $P \uparrow=\Phi$;

(b) $P$ is the largest subclass of ${ }^{\%} X$ subject to the equality $P \uparrow=\Phi$;

(c) $P=\Phi \Downarrow$.

Therefore, $P \uparrow \Downarrow$ is the largest among the subclasses $Q \subset{ }^{\%} X$ subject to $Q \uparrow=P \uparrow$; and $P \uparrow \Downarrow$ is also the only saturated class among these $Q$. In this connection, it is natural to call $P \uparrow \Downarrow$ the saturated hull or the saturation of $P$. The following theorem implies that the saturated hull of a set is a set.

Theorem 4. The following properties of a Boolean-valued class $\Phi \Subset X$ are equivalent:

(a) $\Phi=P \uparrow$ for some set $P \subset{ }^{\%} X$;

(b) $\Phi=P \uparrow$ for some saturated set $P \subset{ }^{\%} X$;

(c) $\Phi=\left(\left.\Phi\right|_{Y}\right) \uparrow$ for some set $Y \subset X$;

(d) $\Phi=\Psi \uparrow$ for some function $\Psi: Y \rightarrow B$, where $Y \subset X$ is a set;

(e) the class $\Phi \Downarrow$ is a set.

Proof. (a) $\Rightarrow$ (c): If $\Phi$ meets (a) then there are families $\left(x_{i}\right)_{i \in I} \subset X$ and $\left(b_{i}\right)_{i \in I} \subset B$, with $I$ a set, such that, for all $x \in X$,

$$
\Phi(x)=\bigvee_{i \in I}\left[x=x_{i}\right] \wedge b_{i}
$$

Then, for all $i \in I$,

$$
\Phi\left(x_{i}\right)=\bigvee_{j \in I}\left[x_{i}=x_{j}\right] \wedge b_{j} \geqslant\left[x_{i}=x_{i}\right] \wedge b_{i}=b_{i},
$$

whence, for every $x \in X$ we have

$$
\Phi(x)=\bigvee_{i \in I}\left[x=x_{i}\right] \wedge b_{i} \leqslant \bigvee_{i \in I}\left[x=x_{i}\right] \wedge \Phi\left(x_{i}\right) \leqslant \Phi(x)
$$

and so $Y:=\left\{x_{i}: i \in I\right\}$ meets (c).

The implication $(\mathrm{c}) \Rightarrow(\mathrm{d})$ is obvious.

$(\mathrm{d}) \Rightarrow(\mathrm{a}):$ If $Y \subset X$ and $\Psi: Y \rightarrow B$ satisfy (d) then $\Phi=\left\{\left.y\right|_{\Psi(y)}: y \in Y\right\} \uparrow$.

(a) $\Rightarrow(\mathrm{e})$ : Let $\Phi=P \uparrow$, where $P \subset{ }^{\%} X$ is a set. According to Lemma 2 (a), we have

$$
\bigvee_{p \in P}[q=p]=[q \in P \uparrow]=[q \in \Phi]=\Delta q
$$


for all $q \in \Phi \Downarrow$. Denote by $F$ the set of all functions $f: P \rightarrow B$ for each of which there exists $q \in \Phi \Downarrow$ such that

$$
(\forall p \in P) f(p)=[q=p] .
$$

The element $q \in \Phi \Downarrow$ is uniquely determined by (12). Indeed, if $q_{1}, q_{2} \in \Phi \Downarrow$ and $\left[q_{1}=p\right]=$ $\left[q_{2}=p\right]$ for all $p \in P$ then, with (11) taken into account,

$$
\begin{gathered}
\Delta q_{1}=\bigvee_{p \in P}\left[q_{1}=p\right]=\bigvee_{p \in P}\left[q_{2}=p\right]=\Delta q_{2}, \\
{\left[q_{1}=q_{2}\right] \geqslant \bigvee_{p \in P}\left[q_{1}=p\right] \wedge\left[q_{2}=p\right]=\bigvee_{p \in P}\left[q_{1}=p\right]=\Delta q_{1},}
\end{gathered}
$$

and so $q_{1}=q_{2}$. Consequently, there is a function $g: F \rightarrow \Phi \Downarrow$ that sends each $f \in F$ to the unique element $q \in \Phi \Downarrow$ subject to (12). It remains to observe that $g$ is surjective, since every $q \in \Phi \Downarrow$ satisfies (12) for the function $f: p \mapsto[q=p]$.

The implication $(\mathrm{e}) \Rightarrow(\mathrm{b})$ follows from Lemma 7 ; and $(\mathrm{b}) \Rightarrow(\mathrm{a})$ is trivial.

Definition 33. Say that a Boolean-valued class $\Phi \Subset X$ is predicative if $\Phi$ possesses each of the equivalent properties (a)-(e) of Theorem 4. The term is based on the fact that the classes that are uniquely determined by sets admit quantification in the first-order predicate language: A phrase starting with the words "for every predicative Boolean-valued class" is not an infinite assertion (see Section 2.4) and can be written as a single formula within predicate calculus (see Section 3.4).

If $\Phi$ and $\Psi$ are Boolean-valued classes and $\Phi \leqslant \Psi$ then $\Phi \Downarrow \subset \Psi \Downarrow$; and so the predicativity of $\Psi$ implies the predicativity of $\Phi$.

Corollary 4. The following properties of $X$ are equivalent:
(a) $X$ is intensional;
(b) the ascents of all saturated subsets of ${ }^{\circ} X$ are represented in $X$;
(c) all predicative Boolean-valued classes are represented in $X$.

Corollary 5. The following properties of an element $x \in X$ are equivalent:
(a) $x \simeq P \uparrow$ for some set $P \subset{ }^{\%} X$;
(b) $x \simeq P \uparrow$ for some saturated set $P \subset{ }^{\%} X$;
(c) $(\exists Y \subset X)(\forall z \in X)[z \in x]=\bigvee_{y \in Y}[z=y] \wedge[y \in x]$;
(d) $x \simeq \Psi \uparrow$ for some function $\Psi: Y \rightarrow B$, where $Y \subset X$ is a set;
(e) the class $x \Downarrow$ is a set.

Definition 34. The elements $x \in X$ subject to the equivalent conditions (a)-(e) of Corollary 5 are called predicative. Therefore, $\mathcal{P}_{X}(X)$ is the totality of all predicative elements of $X$ (see Definition 29). Say that the system $X$ is predicative, if all elements of $X$ are predicative; that is, $\mathcal{P}_{X}(X)=X$.

Definition 35. A Boolean-valued system $X$ is said to satisfy the ascent principle, if $X$ is intensional and predicative:

$$
\begin{aligned}
& \left(\forall P \subset{ }^{\%} X\right)(\exists x \in X)(x \simeq P \uparrow), \\
& (\forall x \in X)\left(\exists P \subset{ }^{\%} X\right)(x \simeq P \uparrow) .
\end{aligned}
$$

Lemma 8. Let $x \in X$ be a predicative element and let $Y \subset X$. Then $[x \subset Y \uparrow]=[x=P \uparrow]$ for some subset $P \subset{ }^{\%} Y$. In particular,

$$
X \vDash(x \subset Y \uparrow) \Leftrightarrow\left(\exists P \subset{ }^{\%} Y\right) x \simeq P \uparrow .
$$

Proof. The statement follows from Lemma 5(c), since the class $P=\left\{\left.y\right|_{[y \in x]}: y \in Y\right\}$ is included in $x \Downarrow$ and so $P$ is a set. 


\subsection{Quantification over Boolean-Valued Classes}

In this subsection, we extend the language of truth values by quantifiers over predicative Boolean-valued classes and show that Boolean-valued classes satisfy an analog of the maximum principle.

Definition 36. Let $\varphi(x)$ be an arbitrary set-theoretic formula and let $X$ be a B-system. Agree to write as $(\forall \Phi \Subset X) \varphi(\Phi)$ and $(\exists \Phi \Subset X) \varphi(\Phi)$ the assertions that $\varphi(\Phi)$ holds for every or, respectively, for some predicative Boolean-valued class $\Phi \Subset X$. Since predicative classes admit quantification (see Definition 33), the assertions are not infinite and each of them can be written down by a single formula:

$$
\begin{aligned}
& (\forall \Phi \Subset X) \varphi(\Phi) \Leftrightarrow\left(\forall P \subset{ }^{\%} X\right) \varphi(P \uparrow), \\
& (\exists \Phi \Subset X) \varphi(\Phi) \Leftrightarrow\left(\exists P \subset{ }^{\%} X\right) \varphi(P \uparrow) .
\end{aligned}
$$

The expressions $\bigwedge_{\Phi \Subset X} \tau(\Phi)$ and $\bigvee_{\Phi \Subset X} \tau(\Phi)$ for a term $\tau(x)$ are defined similarly:

$$
\begin{aligned}
& \bigwedge_{\Phi \Subset X} \tau(\Phi)=\bigwedge_{P \subset{ }^{\%} X} \tau(P \uparrow), \\
& \bigvee_{\Phi \Subset X} \tau(\Phi)=\bigvee_{P \subset{ }^{\%} X} \tau(P \uparrow) .
\end{aligned}
$$

Suppose that $\varphi$ is a formula, $X$ is a $B$-system, and $\Phi \Subset X$. Henceforth, in using the expression $[\varphi(\Phi, \ldots)]_{X}$ or $X \vDash \varphi(\Phi, \ldots)$, we mean that $\varphi$ can have several parameters, some of which are possibly replaced by symbols of Boolean-valued classes; that is, the notation $\varphi(\Phi, \ldots)$ serves as an abbreviation for $\varphi\left(\Phi, y_{1}, \ldots, y_{m}, \Psi_{1}, \ldots, \Psi_{n}\right)$, where $y_{i} \in X$ and $\Psi_{j} \Subset X$ are arbitrary preassigned elements and Boolean-valued classes.

Definition 37. Extend the syntax of Boolean truth values by quantifiers over predicative Booleanvalued classes:

$$
\begin{aligned}
{[(\mathbb{\forall} \Phi) \varphi(\Phi, \ldots)]_{X}: } & :=\bigwedge_{\Phi \Subset X}[\varphi(\Phi, \ldots)]_{X}, \\
{[(\text { 田 } \Phi) \varphi(\Phi, \ldots)]_{X}: } & :=\bigvee_{\Phi \Subset X}[\varphi(\Phi, \ldots)]_{X} .
\end{aligned}
$$

As is easy to see, if $X$ is extensional and satisfies the ascent principle then the quantifiers over classes in $X$ are tantamount to the conventional quantifiers:

$$
\begin{aligned}
& {[(\mathbb{\forall} \Phi) \varphi(\Phi, \ldots)]_{X}=[(\forall x) \varphi(x, \ldots)]_{X},} \\
& {[(\mathbb{H} \Phi) \varphi(\Phi, \ldots)]_{X}=[(\exists x) \varphi(x, \ldots)]_{X} .}
\end{aligned}
$$

The following assertion shows that, in every $B$-system, the Boolean-valued classes satisfy an analog of the maximum principle (see Definition 43).

Theorem 5. Given a formula $\varphi$ and a B-system $X$, the function $\Phi \Subset X \mapsto[\varphi(\Phi, \ldots)] \in B$ attains its maximum:

$$
(\exists \Psi \Subset X)[\varphi(\Psi, \ldots)]=[(\text { 斗 } \Phi) \varphi(\Phi, \ldots)] .
$$

In particular,

$$
\begin{aligned}
& X \vDash(\mathbb{\forall} \Phi) \varphi(\Phi, \ldots) \Leftrightarrow(\forall \Phi \Subset X) X \vDash \varphi(\Phi, \ldots), \\
& X \vDash(\mathbb{H} \Phi) \varphi(\Phi, \ldots) \Leftrightarrow(\exists \Phi \Subset X) X \vDash \varphi(\Phi, \ldots) .
\end{aligned}
$$

Proof. Put

$$
b:=[(\text { 疋 } \Phi) \varphi(\Phi, \ldots)]=\bigvee_{\Phi \Subset X}[\varphi(\Phi, \ldots)] .
$$


By the exhaustion principle (see [4] (2.3.9(2)), [5] (2.1.10(1)), [8] (3.12)), there exist an antichain $\left(d_{i}\right)_{i \in I} \subset B$ and a family of predicative classes $\Phi_{i} \Subset X(i \in I)$ such that $\bigvee_{i \in I} d_{i}=b$ and $d_{i} \leqslant\left[\varphi\left(\Phi_{i}, \ldots\right)\right]$ for all $i \in I$. Define the predicative Boolean-valued class $\Phi: X \rightarrow B$ by putting

$$
\Phi(x):=\bigvee_{i \in I} \Phi_{i}(x) \wedge d_{i}, \quad x \in X
$$

Then, for all $i \in I$ and $x \in X$, we have $\Phi_{i}(x) \wedge d_{i}=\Phi(x) \wedge d_{i}$, whence

$$
\left[\Phi_{i}=\Phi\right]=\left[(\forall x)\left(x \in \Phi_{i} \Leftrightarrow x \in \Phi\right)\right]=\bigwedge_{x \in X}\left(\Phi_{i}(x) \Leftrightarrow_{B} \Phi(x)\right) \geqslant d_{i}
$$

and so, recalling Proposition 11, we conclude that

$$
b=\bigvee_{i \in I} d_{i} \leqslant \bigvee_{i \in I}\left[\varphi\left(\Phi_{i}, \ldots\right)\right] \wedge\left[\Phi_{i}=\Phi\right] \leqslant[\varphi(\Phi, \ldots)]
$$

Lemma 9. Suppose that $\varphi$ is a formula, $X$ is a $B$-system, and $\Psi \Subset X$. Then

$$
[(\mathbb{\forall} \Phi \subset \Psi) \varphi(\Phi, \ldots)]=[(\mathbb{\forall} \Phi) \varphi(\Phi \cap \Psi, \ldots)]=\bigwedge_{\Phi \Subset X: \Phi \leqslant \Psi}[\varphi(\Phi, \ldots)] .
$$

In particular, the following are equivalent:

(a) $X \vDash(\mathbb{\forall} \Phi \subset \Psi) \varphi(\Phi, \ldots)$;

(b) $X \vDash(\mathbb{V} \Phi) \varphi(\Phi \cap \Psi, \ldots)$;

(c) $(\forall \Phi \Subset X)(\Phi \leqslant \Psi \Rightarrow X \vDash \varphi(\Phi, \ldots))$.

Proof. Given a predicative class $\Phi \Subset X$, define $\Phi_{\Psi} \Subset X$ by putting

$$
\Phi_{\Psi}(x):=\Phi(x) \wedge \Psi(x), \quad x \in X .
$$

Then $\Phi_{\Psi} \leqslant \Psi$ and $\left[\Phi_{\Psi} \subset \Psi\right]=\left[\Phi_{\Psi}=\Phi \cap \Psi\right]=1_{B}$. Since

$$
\left[\Phi_{\Psi} \subset \Psi \Rightarrow \varphi\left(\Phi_{\Psi}, \ldots\right)\right]=\left[\varphi\left(\Phi_{\Psi}, \ldots\right)\right]=[\varphi(\Phi \cap \Psi, \ldots)] ;
$$

therefore,

$$
\bigwedge_{\Phi \Subset X}[\Phi \subset \Psi \Rightarrow \varphi(\Phi, \ldots)] \leqslant \bigwedge_{\Phi \Subset X}[\varphi(\Phi \cap \Psi, \ldots)] .
$$

Furthermore, if $\Phi \leqslant \Psi$ then $[\Phi \cap \Psi=\Phi]=1_{B}$ and $[\varphi(\Phi \cap \Psi, \ldots)]=[\varphi(\Phi, \ldots)]$, and so

$$
\bigwedge_{\Phi \Subset X}[\varphi(\Phi \cap \Psi, \ldots)] \leqslant \bigwedge_{\Phi \Subset X: \Phi \leqslant \Psi}[\varphi(\Phi \cap \Psi, \ldots)]=\bigwedge_{\Phi \Subset X: \Phi \leqslant \Psi}[\varphi(\Phi, \ldots)] .
$$

Finally, the relations

$$
\begin{aligned}
& {[\varphi(\Phi \cap \Psi, \ldots) \Rightarrow(\Phi \subset \Psi \Rightarrow \varphi(\Phi, \ldots))]} \\
& \quad=[(\varphi(\Phi \cap \Psi, \ldots) \wedge \Phi \cap \Psi=\Phi) \Rightarrow \varphi(\Phi, \ldots)]=1_{B}
\end{aligned}
$$

imply that

$$
\left[\varphi\left(\Phi_{\Psi}, \ldots\right)\right]=[\varphi(\Phi \cap \Psi, \ldots)] \leqslant[\Phi \subset \Psi \Rightarrow \varphi(\Phi, \ldots)]
$$

and so

$$
\bigwedge_{\Phi \Subset X: \Phi \leqslant \Psi}[\varphi(\Phi, \ldots)] \leqslant \bigwedge_{\Phi \Subset X}[\Phi \subset \Psi \Rightarrow \varphi(\Phi, \ldots)] .
$$

\subsection{Joins, Mixings, and Cyclicity}

In this subsection, we study the joins of antichains of partial elements, cyclic subclasses, and the mixings of subclasses in a Boolean-valued system. 
In what follows, $B$ is a complete Boolean algebra and $X$ is a $B$-system.

Recall that elements $a, b \in B$ are disjoint, in writing $a \perp b$, whenever $a \wedge b=0_{B}$. For $A \subset B$, the relation $(\forall a \in A)(a \perp b)$ is abbreviated as $A \perp b$. An antichain in a Boolean algebra is a set or family of pairwise disjoint elements, while a partition of unity is a maximal antichain, that is, an antichain whose supremum equals $1_{B}$.

The following simple observations are repeatedly employed in the sequel (see, e.g., Lemmas 11 and 13).

\section{Lemma 10.}

(a) If $\left(d_{i}\right)_{i \in I} \subset B$ is an antichain, $\left(b_{i}\right)_{i \in I} \subset B,(\forall i \in I)\left(b_{i} \leqslant d_{i}\right)$, and $\bigvee_{i \in I} b_{i}=\bigvee_{i \in I} d_{i}$; then $(\forall i \in I)\left(b_{i}=d_{i}\right)$.

(b) If $A \subset B, \bar{a} \in B$, and $A \leqslant \bar{a}$; then $\vee A=\bar{a} \Leftrightarrow(\forall b \in B)(A \perp b \Rightarrow \bar{a} \perp b)$.

Definition 38. Define the descent $\Phi \downarrow$ of a Boolean-valued class $\Phi \Subset X$ as follows:

$$
\begin{aligned}
\Phi \downarrow & =\left\{p \in{ }^{\%} X:[p \in \Phi]=\Delta p=\vee \Phi\right\} \\
& =\{p \in \Phi \Downarrow:[p \in \Phi]=\vee \Phi\},
\end{aligned}
$$

where $\vee \Phi:=\bigvee_{x \in X} \Phi(x)=[\Phi \neq \varnothing]$. It is clear that

$$
\Phi \downarrow=\left\{\left.x\right|_{\vee \Phi}: x \in X, \Phi(x)=\vee \Phi\right\} ;
$$

therefore, $\Phi \downarrow \neq \varnothing$ if and only if $\Phi$ attains its maximum (see Definition 43). The descent $x \downarrow$ of an element $x \in X$ is defined as the descent $[\cdot \in x] \downarrow$ of the Boolean-valued class $[\cdot \in x]$ (see Definition 27). Observe that $\Phi \downarrow$ and $x \downarrow$ can be proper classes. If $X \vDash(\Phi \neq \varnothing)$ and $X$ is separated; then, according to the agreement of Definition 24,

$$
\Phi \downarrow=\{x \in X: X \vDash(x \in \Phi)\} .
$$

Definition 39. Say that a subset $P \subset{ }^{\%} X$ is an antichain if the elements of $P$ have pairwise disjoint domains: $\Delta p \perp \Delta q$ for all $p, q \in P, p \neq q$. A partial element $q \in \% X$ is the join of an antichain $P \subset{ }^{\%} X$ provided that

$$
\Delta q=\bigvee_{p \in P} \Delta p \text { and }(\forall p \in P) p \sqsubset q .
$$

As is easily seen, $q$ is uniquely determined by $P$, which fact justifies the notation $\sqcup P$ for the join of $P$. The join $\sqcup\{p, q\}$ of a two-element antichain $\{p, q\} \subset{ }^{\%} X$ will be written as $p \sqcup q$, while the join $\sqcup\left\{p_{i}: i \in I\right\}$ of the antichain defined by a family $\left(p_{i}\right)_{i \in I} \subset{ }^{\%} X$, as $\bigsqcup_{i \in I} p_{i}$.

Proposition 14. Inside $X$, the ascent of an antichain $P \subset{ }^{\%} X$ contains at most one element: $X \vDash$ $(\forall x, y \in P \uparrow)(x=y)$.

Proof. By Lemma 6(b) we have

$$
\begin{gathered}
{[(\forall x, y \in P \uparrow)(x=y)]=\bigwedge_{p, q \in P}[p=q] \vee \neg \Delta p \vee \neg \Delta q} \\
=\neg \bigvee_{p, q \in P}[p \neq q] \wedge \Delta p \wedge \Delta q=\neg \bigvee_{p \in P}[p \neq p]=1_{B} .
\end{gathered}
$$

The next assertion is easily verified by means of Lemma 10 (a) and Proposition 14 .

Lemma 11. If $P \subset \% X$ is an antichain, $x \in X$, and $b:=[P \uparrow \neq \varnothing]$, then the following are equivalent:
(a) $\left.x\right|_{b}=\sqcup P$;
(b) $(\forall p \in P)[x=p]=\Delta p$;
(c) $\vee_{p \in P}[x=p]=b$;
(d) $[x \in P \uparrow]=b$;
(e) $[P \uparrow=\{x\}]=b$; 
(f) $\left.x\right|_{b} \in P \uparrow \downarrow ;$

(g) $P \uparrow \downarrow=\left\{\left.x\right|_{b}\right\}$.

Corollary 6. If $P \subset{ }^{\%} X$ is an antichain and $P \uparrow \downarrow \neq \varnothing$, then the join $\sqcup P$ exists and $P \uparrow \downarrow=\{\sqcup P\}$.

Definition 40. Call a global partial element $\left.\bar{p} \in X\right|_{1_{B}}=\widetilde{X}$ the least global extension of a partial element $p \in{ }^{\%} X$ if $p \sqsubset \bar{p}$ and $[\bar{p}=\varnothing] \geqslant \neg \Delta p$ or, which is equivalent,

$$
[\bar{p} \neq \varnothing] \leqslant[\bar{p}=p]=\Delta p .
$$

The term is justified by the fact that $\bar{p}$ is the inclusion least inside $X$ among all global extensions of $p$; that is, $(\forall q \in \widetilde{X})(p \sqsubset q \Rightarrow X \vDash \bar{p} \subset q)$.

The relation (13) implies that, for all $z \in X$,

$$
\begin{aligned}
{[z \in \bar{p}] } & =[(z \in \bar{p}) \wedge(\bar{p} \neq \varnothing)]=[z \in \bar{p}] \wedge[\bar{p} \neq \varnothing] \wedge[\bar{p}=p] \\
& =[z \in p] \wedge[p \neq \varnothing] \wedge \Delta p=[z \in p] ;
\end{aligned}
$$

and so, if $X$ is extensional, then the least global extension $\bar{p}$ of a partial element $p$ is unique. In this case we denote $\bar{p}$ by ext $p$. If, moreover, $X$ is separated; then ext $p \in X$ according to the agreement of Definition 24.

Lemma 12. If $X$ is an extensional $B$-system, $P \subset{ }^{\%} X$ is an antichain, and $x \in X$, then the following are equivalent:

(a) $\widetilde{x}=\operatorname{ext} \sqcup P$;

(b) $X \vDash(x=\cup(P \uparrow))$;

(c) $[x \neq \varnothing] \leqslant[P \uparrow \neq \varnothing] \leqslant[x \in P \uparrow]$;

(d) $(\forall z \in X)[z \in x]=\bigvee_{p \in P}[z \in p]$.

Proof. The equivalence of (a)-(c) is straightforward from the definitions. we have

(a) $\Rightarrow(d)$ : For all $z \in X$, due to the relations $[z \in x] \leqslant[x \neq \varnothing] \leqslant \bigvee_{p \in P} \Delta p$ and $\left.x\right|_{\Delta p}=p$,

$$
[z \in x]=\bigvee_{p \in P}[z \in x] \wedge \Delta p=\bigvee_{p \in P}[z \in p]
$$

(d) $\Rightarrow$ (c): If $p \in P$ then $(\forall z \in X)[z \in x] \wedge \Delta p \geqslant[z \in p]$ according to (d). Whence, with Lemma 10 (a) taken into account, it follows that $(\forall z \in X)[z \in x] \wedge \Delta p=[z \in p]$ and so $[x=p] \geqslant \Delta p$ by extensionality of $X$. Consequently,

$$
[x \neq \varnothing]=\bigvee_{z \in X}[z \in x]=\bigvee_{z \in X} \bigvee_{p \in P}[z \in p] \leqslant \bigvee_{p \in P} \Delta p \leqslant \bigvee_{p \in P}[x=p]=[x \in P \uparrow]
$$

Definition 41. An antichain $P \subset{ }^{\%} X$ is maximal whenever $\bigvee_{p \in P} \Delta p=1_{B}$; that is, the domains of the elements of $P$ form a partition of unity in $B$. The joins of maximal antichains are global partial elements of $X$; and, if $X$ is separated, are identified with the corresponding elements of $X$ (see Definition 24).

If $x \in X,\left\{\left.y_{i}\right|_{d_{i}}: i \in I\right\}$ is a maximal antichain, and $\widetilde{x}=\left.\bigsqcup_{i \in I} y_{i}\right|_{d_{i}}$; then $\widetilde{x}$ is called the mixing of $\left(y_{i}\right)_{i \in I} \subset X$ with respect to the partition of unity $\left(d_{i}\right)_{i \in I} \subset B$. As is easily seen,

$$
\widetilde{x}=\left.\bigsqcup_{i \in I} y_{i}\right|_{d_{i}} \Leftrightarrow(\forall i \in I)\left[x=y_{i}\right] \geqslant d_{i} .
$$

In the case of a separated system $X$, the mixing $\left.\bigsqcup_{i \in I} y_{i}\right|_{d_{i}}$, regarded as an element of $X$, is denoted by $\operatorname{mix}_{i \in I} d_{i} y_{i}$.

The next two assertions follow from Proposition 14 and Lemma 11. 
Proposition 15. Inside $X$, the ascent of an arbitrary maximal antichain $P \subset{ }^{\%} X$ is a singleton: $X \vDash(\exists ! x)(x \in P \uparrow)$.

Lemma 13. If $P \subset{ }^{\%} X$ is a maximal antichain and $x \in X$, then the following are equivalent:
(a) $\tilde{x}=\sqcup P$;
(b) $(\forall p \in P)[x=p]=\Delta p$;
(c) $\bigvee_{p \in P}[x=p]=1_{B}$;
(d) $X \vDash(x \in P \uparrow)$;
(e) $X \vDash(P \uparrow=\{x\})$;
(f) $\tilde{x} \in P \uparrow \downarrow ;$
(g) $P \uparrow \downarrow=\{\tilde{x}\}$.

Definition 42. A subclass $Y \subset X$ is called cyclic whenever, for every family $\left(y_{i}\right)_{i \in I} \subset Y$ and every partition of unity $\left(d_{i}\right)_{i \in I} \subset B$, there exists an element $y \in Y$ such that $\widetilde{y}=\left.\bigsqcup_{i \in I} y_{i}\right|_{d_{i}}$. If the entire B-system $X$ is cyclic, then $X$ is said to satisfy the mixing principle.

Lemma 14. The following properties of a nonempty class $Y \subset X$ are equivalent:
(a) $Y$ is cyclic;
(b) every antichain $P \subset{ }^{\%} Y$ has a join $\sqcup P \in{ }^{\%} Y$;
(c) every maximal antichain $P \subset{ }^{\%} Y$ has a join $\sqcup P \in \widetilde{Y}$.

Lemma 15. Let $Y \subset X$. The following properties of an element $x \in X$ are equivalent:
(a) $\tilde{x}=\left.\bigsqcup_{i \in I} y_{i}\right|_{d_{i}}$ for some family $\left(y_{i}\right)_{i \in I} \subset Y$ and partition of unity $\left(d_{i}\right)_{i \in I} \subset B$;
(b) $\tilde{x}=\sqcup P$ for some maximal antichain $P \subset{ }^{\%} Y$;
(c) $X \vDash(x \in Y \uparrow)$;
(d) $\tilde{x} \in Y \uparrow \downarrow$;
(e) $\bigvee_{y \in Y}[x=y]=1_{B}$.

Proof. The implications $(\mathrm{a}) \Rightarrow(\mathrm{b})$ and $(\mathrm{c}) \Rightarrow(\mathrm{d}) \Rightarrow(\mathrm{e})$ are obvious. Furthermore, $(\mathrm{b}) \Rightarrow(\mathrm{c})$ follows from Lemmas 13 and $5(\mathrm{~b})$, while $(\mathrm{e}) \Rightarrow(\mathrm{a})$ is justified by the exhaustion principle.

The totality of elements $x \in X$ possessing each of the equivalent properties (a)-(e) is denoted by mix $Y$. Note that, if the system is not separated, then $\operatorname{mix} Y$ can be a proper class even if $Y$ is a set.

Lemma 16. If $Y \subset X$ then $Y \uparrow=(\operatorname{mix} Y) \uparrow$. In particular, for every Boolean-valued class $\Phi \Subset X$,

$$
\bigvee_{y \in Y} \Phi(y)=[(\exists y \in Y \uparrow) \Phi(y)]=[(\exists x \in(\operatorname{mix} Y) \uparrow) \Phi(x)]=\bigvee_{x \in \operatorname{mix} Y} \Phi(x)
$$

Proof. The inequality $Y \uparrow \leqslant(\operatorname{mix} Y) \uparrow$ is obvious. On the other hand, for all $x \in X$ and $z \in \operatorname{mix} Y$,

$$
[x=z]=[x=z] \wedge \bigvee_{y \in Y}[z=y]=\bigvee_{y \in Y}[x=z] \wedge[z=y] \leqslant \bigvee_{y \in Y}[x=y]=Y \uparrow(x),
$$

whence $(\operatorname{mix} Y) \uparrow(x)=\bigvee_{z \in \operatorname{mix} Y}[x=z] \leqslant Y \uparrow(x)$.

Lemma 17. If $Y \subset X$ then $\operatorname{mix} \operatorname{mix} Y=\operatorname{mix} Y$.

Proof. With Lemma 16 taken into account, for all $x \in X$ we have

$$
x \in \operatorname{mix} \operatorname{mix} Y \Leftrightarrow X \vDash(x \in(\operatorname{mix} Y) \uparrow) \Leftrightarrow X \vDash(x \in Y \uparrow) \Leftrightarrow x \in \operatorname{mix} Y .
$$

Corollary 7. The following properties of a subclass $Y \subset X$ are equivalent: 
(a) if $\left(y_{i}\right)_{i \in I} \subset Y$ and $\left(d_{i}\right)_{i \in I} \subset B$ is a partition of unity, then there is a mixing $\left.\bigsqcup_{i \in I} y_{i}\right|_{d_{i}} \in \widetilde{X}$;

(b) each antichain $P \subset{ }^{\%} Y$ has a join $\sqcup P \in{ }^{\%} X$;

(c) each maximal antichain $P \subset{ }^{\%} Y$ has a join $\sqcup P \in \widetilde{X}$;

(d) $Y \subset \bar{Y}$ for some cyclic class $\bar{Y} \subset X$;

(e) mix $Y$ is a cyclic class.

A class $Y$ subject to each of the equivalent conditions (a)-(e) will be called precyclic.

Remark 14. If $Y$ is a precyclic subclass of $X$, then

$$
Y \text { is cyclic } \Leftrightarrow \widetilde{Y}=(\operatorname{mix} Y)^{\sim} \Leftrightarrow \widetilde{Y}=Y \uparrow \downarrow .
$$

In the case of a separated system, the latter turns into the equality $Y=Y \uparrow \downarrow$, which originates the term "cyclic." If $Y$ is a precyclic subset of a separated system $X$, then mix $Y$ is the inclusion least cyclic subset of $X$ including $Y$. In this case, mix $Y$ is called the cyclic hull of $Y$.

Lemma 18. If $X$ satisfies the mixing principle; then, for all families $\left(x_{i}\right)_{i \in I} \subset X$ and $\left(b_{i}\right)_{i \in I} \subset B$, there are $Y \subset \operatorname{mix}\left\{x_{i}: i \in I\right\}$ and $b \in B$ such that

$$
\left\{\left.x_{i}\right|_{b_{i}}: i \in I\right\} \uparrow=\left(\left.Y\right|_{b}\right) \uparrow .
$$

Proof. In the case of $I=\varnothing$, the assertion is obvious. Suppose that $I \neq \varnothing$.

Put $b=\bigvee_{i \in I} b_{i}, I^{\bullet}=I \cup\{I\}, b_{I}=\neg b$, and take an arbitrary element $x_{I} \in\left\{x_{i}: i \in I\right\}$. By the exhaustion principle, there exists a partition of unity $\left(d_{i}\right)_{i \in I^{\bullet}} \subset B$ such that $d_{i} \leqslant b_{i}$ for all $i \in I^{\bullet}$. Observe that $\bigvee_{i \in I} d_{i}=b$. Owing to cyclicity of $X$, there is an element $\left.x \sim \bigsqcup_{i \in I} \bullet x_{i}\right|_{d_{i}}$ determined by the relations

$$
\left[x=x_{i}\right] \geqslant d_{i} \quad \text { for all } i \in I^{\bullet} .
$$

For the same reason, for each $i \in I$ there is an element $\left.\left.y_{i} \sim x_{i}\right|_{b_{i}} \sqcup x\right|_{\neg b_{i}}$ in $X$ subject to the inequalities

$$
\left[y_{i}=x_{i}\right] \geqslant b_{i}, \quad\left[y_{i}=x\right] \geqslant \neg b_{i} .
$$

Put $Y=\left\{y_{i}: i \in I\right\}$. Obviously, $Y \subset \operatorname{mix}\left\{x_{i}: i \in I\right\}$. Show that $\left\{\left.x_{i}\right|_{b_{i}}: i \in I\right\} \uparrow=\left(\left.Y\right|_{b}\right) \uparrow$, that is,

$$
\bigvee_{i \in I}\left[z=x_{i}\right] \wedge b_{i}=\bigvee_{i \in I}\left[z=y_{i}\right] \wedge b \text { for all } z \in X
$$

For every $z \in X$, with (15) taken into account, we have

$$
\bigvee_{i \in I}\left[z=x_{i}\right] \wedge b_{i}=\bigvee_{i \in I}\left[z=y_{i}\right] \wedge b_{i} \leqslant \bigvee_{i \in I}\left[z=y_{i}\right] \wedge b
$$

On the other hand, due to (14), the following holds:

$$
[z=x] \wedge b=\bigvee_{i \in I}[z=x] \wedge d_{i}=\bigvee_{i \in I}\left[z=x_{i}\right] \wedge d_{i} \leqslant \bigvee_{i \in I}\left[z=x_{i}\right] \wedge b_{i}
$$

whence, according to (15), we conclude that, for all $i \in I$,

$$
\begin{aligned}
{\left[z=y_{i}\right] \wedge b } & =\left(\left[z=y_{i}\right] \wedge b_{i}\right) \vee\left(\left[z=y_{i}\right] \wedge b \wedge \neg b_{i}\right) \\
& =\left(\left[z=x_{i}\right] \wedge b_{i}\right) \vee\left([z=x] \wedge b \wedge \neg b_{i}\right) \\
& \leqslant\left(\left[z=x_{i}\right] \wedge b_{i}\right) \vee \bigvee_{j \in I}\left[z=x_{j}\right] \wedge b_{j}=\bigvee_{j \in I}\left[z=x_{j}\right] \wedge b_{j},
\end{aligned}
$$

and, therefore, $\bigvee_{i \in I}\left[z=y_{i}\right] \wedge b \leqslant \bigvee_{i \in I}\left[z=x_{i}\right] \wedge b_{i}$.

Corollary 8. Suppose that $X$ is a B-system satisfying the mixing principle. 
(a) For all $Z \subset X$ and $P \subset{ }^{\%} Z$, there are $Y \subset$ mix $Z$ and $b \in B$ such that $P \uparrow=\left(\left.Y\right|_{b}\right) \uparrow$.

(b) For every predicative element $x \in X$, there exist $Y \subset X$ and $b \in B$ such that $x \simeq\left(\left.Y\right|_{b}\right) \uparrow$. In this event, $b=[x \neq \varnothing]=[y \in x]$ for all $y \in Y$.

(c) If $(\forall Y \subset X)(\forall b \in B)(\exists x \in X) x \simeq\left(\left.Y\right|_{b}\right) \uparrow$ then $X$ is intensional.

\subsection{The Maximum Principle}

In this subsection, we examine the maximum principle and its relationship with the ascent and mixing principles.

Let $B$ be a complete Boolean algebra and let $X$ be an arbitrary $B$-system.

Definition 43. Say that a Boolean-valued class $\Phi \Subset X$ attains its maximum on $Y \subset X$ whenever the set $\{\Phi(y): y \in Y\}$ has a top, that is,

$$
(\exists z \in Y) \Phi(z)=[(\exists y \in Y \uparrow) \Phi(y)] .
$$

Say that $\Phi$ attains its maximum, if $\Phi$ attains its maximum on $X$ or, which is the same, $\Phi \downarrow \neq \varnothing$ (see Definition 38).

Let $\varphi(x, \ddot{z})$ be a formula whose parameters are contained in the list $x, \ddot{z}$. The system $X$ is said to satisfy the maximum principle for the formula $(\exists x) \varphi$ provided that, for all $\ddot{z} \in X$, the Boolean-valued class $x \mapsto[\varphi(x, \ddot{z})]$ attains its maximum, that is,

$$
(\forall \ddot{z} \in X)(\exists y \in X)[\varphi(y, \ddot{z})]=[(\exists x) \varphi(x, \ddot{z})] .
$$

Say that $X$ satisfies the maximum principle if $X$ satisfies the maximum principle for every formula $(\exists x) \varphi$. (The latter is an infinite assertion; see Section 2.4.)

Lemma 19. A Boolean-valued class $\Phi \Subset X$ satisfies the relation $\Phi=\Phi \downarrow \uparrow$ if and only if

$$
(\forall p \in \Phi \downarrow)([x=p] \perp b) \Rightarrow \Phi(x) \perp b
$$

for all $x \in X$ and $b \in B$.

Proof. If $x \in X$ and $p \in \Phi \downarrow$ then $p=\left.y\right|_{\Phi(y)}$ for some $y \in X$ and, by extensionality of $\Phi$,

$$
[x=p]=\left[x=\left.y\right|_{\Phi(y)}\right]=[x=y] \wedge \Phi(y)=[x=y] \wedge \Phi(x) \leqslant \Phi(x) .
$$

Since $\Phi \downarrow \uparrow(x)=\bigvee_{p \in \Phi \downarrow}[x=p]$, it remains to refer to Lemma 10 (b).

Lemma 20. Let $\Phi \Subset X$. For $x \in X$, define the extensional function $\Phi_{x}: X \rightarrow B$ by putting

$$
\Phi_{x}(y)=(\neg \Phi(x) \vee[x=y]) \wedge \Phi(y), \quad y \in X .
$$

Then the following are equivalent:

(a) $(\forall p \in \Phi \Downarrow)(\exists \bar{p} \in \Phi \downarrow)(p \sqsubset \bar{p})$;

(b) $(\forall x \in X)(\exists y \in X)(\Phi(x) \leqslant[x=y], \Phi(y)=\vee \Phi)$;

(c) $(\forall x \in X)\left(\Phi_{x \downarrow} \neq \varnothing\right)$.

If $\Phi$ possesses any of the equivalent properties (a)-(c) then

$$
\Phi=\Phi \downarrow \uparrow .
$$

Proof. Show first that $\vee \Phi_{x}=\vee \Phi$. Indeed, the inequality $\vee \Phi_{x} \leqslant \vee \Phi$ is obvious, while, on the other hand, for all $y \in X$,

$$
\vee \Phi_{x} \geqslant \Phi_{x}(x) \vee \Phi_{x}(y)=\Phi(x) \vee((\neg \Phi(x) \vee[x=y]) \wedge \Phi(y))=\Phi(x) \vee \Phi(y) \geqslant \Phi(y) .
$$


(a) $\Leftrightarrow(b)$ : It suffices to observe that

$$
\begin{aligned}
& \left.x\right|_{b} \in \Phi \Downarrow \Leftrightarrow b \leqslant \Phi(x),\left.\quad y\right|_{\vee \Phi} \in \Phi \downarrow \Leftrightarrow \Phi(y)=\vee \Phi,\left.\left.\quad x\right|_{\Phi(x)} \sqsubset y\right|_{\vee} \Leftrightarrow \Leftrightarrow \Phi(x) \leqslant[x=y] . \\
& (b) \Rightarrow(c): \text { If } \Phi(x) \leqslant[x=y] \text { and } \Phi(y)=\vee \Phi \text { then } \\
& \Phi_{x}(y)=(\neg \Phi(x) \vee[x=y]) \wedge \Phi(y) \geqslant(\neg \Phi(x) \vee \Phi(x)) \wedge \Phi(y)=\Phi(y)=\vee \Phi=\vee \Phi_{x} ;
\end{aligned}
$$

and so $\Phi_{x}$ attains its maximum at $y$.

(c) $\Rightarrow(b)$ : Let $x \in X$ and let $y \in X$ be a maximum point of $\Phi_{x}$; that is,

$$
(\neg \Phi(x) \vee[x=y]) \wedge \Phi(y)=\vee \Phi_{x}=\vee \Phi .
$$

Since $\Phi(y) \leqslant \vee \Phi$, from (17) it follows that $\Phi(y)=\vee \Phi$. Consequently,

$$
(\neg \Phi(x) \vee[x=y]) \wedge \vee \Phi=\vee \Phi,
$$

whence, $\neg \Phi(x) \vee[x=y] \geqslant \vee \Phi \geqslant \Phi(x)$ and so $\Phi(x) \leqslant[x=y]$.

Suppose that $\Phi$ meets (b) and demonstrate that $\Phi=\Phi \downarrow \uparrow$. According to Lemma 19, it suffices to consider $x \in X$ and $b \in B$ subject to the condition

$$
(\forall y \in Y)(\Phi(y)=\vee \Phi \Rightarrow[x=y] \wedge \vee \Phi \perp b)
$$

and show that $\Phi(x) \perp b$. By (b), there is $y \in X$ such that $\Phi(x) \leqslant[x=y]$ and $\Phi(y)=\vee \Phi$. Then, with (18) taken into account, we have

$$
\Phi(x)=\Phi(x) \wedge \vee \Phi \leqslant[x=y] \wedge \vee \Phi \perp b .
$$

Remark 15. Observe that conditions (a)-(c) of Lemma 20 are not equivalent to the equality $\Phi=\Phi \downarrow \uparrow$. For instance, if

$$
\begin{aligned}
& B=\mathcal{P}(\{0,1,2\}), \\
& X=\{(1,0,0),(0,1,0),(1,1,1)\}, \\
& {[=](x, y)=\{i \in\{0,1,2\}: x(i)=y(i)\},} \\
& {[\in](x, y)=\{i \in\{0,1,2\}: x(i) \in y(i)\}, \text { where } 0 \in\{0\}=1,} \\
& \Phi(1,0,0)=\Phi(0,1,0)=\{0,1,2\}, \Phi(1,1,1)=\{0,1\},
\end{aligned}
$$

then $\Phi=\Phi \downarrow \uparrow$, but for $x=(1,1,1)$ there is no $y \in X$ subject to the conditions $\Phi(x) \leqslant[x=y]$ and $\Phi(y)=\vee \Phi$.

Proposition 16. Suppose that the B-system X satisfies the maximum principle for the formula $(\exists y)((z \in x \Rightarrow z=y) \wedge y \in x)$. Then

(a) $x \simeq x \downarrow \uparrow$ for all $x \in X$;

(b) $x \in X$ is predicative if and only if the class $x \downarrow$ is a set (see Corollary 5).

Proof. By applying Lemma 20 to $\Phi=[\cdot \in x]$ and $\Phi_{z}(y)=[(z \in x \Rightarrow z=y) \wedge y \in x]$, we see that (a) follows from Lemma 20 (c), while (b) follows from Corollary 5 (e), Lemma 20 (a) and the inclusion $x \downarrow \subset x \Downarrow$.

Theorem 6. The following properties of a nonempty subclass $Y \subset X$ are equivalent:

(a) Y is cyclic;

(b) each Boolean-valued class $\Phi \Subset X$ attains its maximum on $Y$;

(c) each predicative Boolean-valued class $\Phi \Subset X$ attains its maximum on $Y$. 
Proof. (a) $\Rightarrow$ (b): Put $b:=\bigvee_{y \in Y} \Phi(y)$. By the exhaustion principle, there exist an antichain $\left(d_{i}\right)_{i \in I} \subset B$ and a family $\left(y_{i}\right)_{i \in I} \subset Y$ such that $\bigvee_{i \in I} d_{i}=b$ and $d_{i} \leqslant \Phi\left(y_{i}\right)$ for all $i \in I$. Owing to cyclicity of $Y$, there is an element $z \in Y$ subject to the condition $\left.z\right|_{b}=\left.\bigsqcup_{i \in I} y_{i}\right|_{d_{i}}$. Show that $\Phi(z)=b$. Indeed, $\Phi(z)=\bigvee_{y \in Y}[z=y] \wedge \Phi(z) \leqslant \bigvee_{y \in Y} \Phi(y)=b$. On the other hand, for all $i \in I$, with account taken of the inequalities $\left[z=y_{i}\right] \geqslant d_{i}, \Phi\left(y_{i}\right) \geqslant d_{i}$, we have $\Phi(z) \geqslant \Phi\left(y_{i}\right) \wedge\left[z=y_{i}\right] \geqslant d_{i}$ and, hence, $\Phi(z) \geqslant \bigvee_{i \in I} d_{i}=b$.

The implication $(b) \Rightarrow(c)$ is trivial.

(c) $\Rightarrow(\mathrm{a})$ : Let $P \subset{ }^{\%} Y$ be an arbitrary maximal antichain. By (c), there exists an element $z \in Y$ such that $P \uparrow(z)=\bigvee_{y \in Y} P \uparrow(y)$. According to the assertions (b) and (c) of Lemma 2, we have $\bigvee_{y \in Y} P \uparrow(y)=1_{B}$. Consequently, $[z \in P \uparrow]=1_{B}$; whence, it follows by Lemma 13 that $\widetilde{z}=\sqcup P$.

Theorem 7. The following properties of a B-system $X$ are equivalent:

(a) $X$ satisfies the mixing principle;

(b) each Boolean-valued class $\Phi \Subset X$ attains its maximum;

(c) each predicative Boolean-valued class $\Phi \Subset X$ attains its maximum;

(d) $\Phi=\Phi \downarrow \uparrow$ for every Boolean-valued class $\Phi \Subset X$;

(e) $\Phi=\Phi \downarrow \uparrow$ for every predicative Boolean-valued class $\Phi \Subset X$;

(f) $P \uparrow \downarrow \neq \varnothing$ for every class $P \subset{ }^{\%} X$;

(g) $P \uparrow \downarrow \neq \varnothing$ for every set $P \subset \%$;

(h) $P \uparrow \downarrow \neq \varnothing$ for every maximal antichain $P \subset{ }^{\%} X$.

Proof. The equivalence of (a)-(c) is established in Theorem 6. The implications (d) $\Rightarrow(f)$ and (e) $\Rightarrow$ (g) are obvious: if $\Phi:=P \uparrow$ and $\Phi \downarrow=\varnothing$ then $\Phi \downarrow \uparrow \downarrow=\varnothing \uparrow \downarrow=\left\{\left.x\right|_{0_{B}}\right\} \neq \varnothing=\Phi \downarrow$ and, hence, $\Phi \downarrow \uparrow \neq \Phi$. The implication (b) $\Rightarrow(\mathrm{d})$ follows from Lemma 20 (c) $\Rightarrow(16)$; the implication $(\mathrm{h}) \Rightarrow(\mathrm{a})$ follows from Corollary 6 ; while $(\mathrm{d}) \Rightarrow(\mathrm{e})$ and $(\mathrm{f}) \Rightarrow(\mathrm{g}) \Rightarrow(\mathrm{h})$ are trivial.

Lemma 21. If $Y \subset X$ is a nonempty precyclic class then

$$
(\forall x \in X)(\exists y \in \operatorname{mix} Y)[x=y]=[x \in Y \uparrow] .
$$

Proof. According to Theorem 6, the Boolean-valued class $y \mapsto[x=y]$ attains its maximum on mix $Y$ and, moreover, $(\operatorname{mix} Y) \uparrow=Y \uparrow($ see Lemma 16$)$.

Lemma 22. Suppose that $X$ is an extensional B-system, $\left(d_{i}\right)_{i \in I} \subset B$ is an antichain, $\left(P_{i}\right)_{i \in I}$ is a family of subsets of ${ }^{\%}$, and $\left(x_{i}\right)_{i \in I} \subset X$. If $(\forall i \in I) x_{i} \simeq P_{i} \uparrow$ and $x \simeq\left(\left.\bigcup_{i \in I} P_{i}\right|_{d_{i}}\right) \uparrow$ then

$$
\widetilde{x}=\left.\operatorname{ext} \bigsqcup_{i \in I} x_{i}\right|_{d_{i}} .
$$

Proof. The claim follows from Lemma 12, since, for all $z \in X$,

$$
\begin{aligned}
{[z \in x] } & =\left[z \in\left(\bigcup_{i \in I} P_{i} \mid d_{i}\right) \uparrow\right]=\bigvee_{i \in I} \bigvee_{p \in P_{i}}\left[z=\left.p\right|_{d_{i}}\right]=\bigvee_{i \in I} \bigvee_{p \in P_{i}}[z=p] \wedge d_{i} \\
& =\bigvee_{i \in I}\left[z \in P_{i} \uparrow\right] \wedge d_{i}=\bigvee_{i \in I}\left[z \in x_{i}\right] \wedge d_{i}=\bigvee_{i \in I}\left[\left.z \in x_{i}\right|_{d_{i}}\right] .
\end{aligned}
$$

Corollary 9. Let $X$ be an extensional B-system. If $Y \subset X$ and $(\forall P \subset \% Y)(\exists x \in X)(x \simeq P \uparrow)$ then $\mathcal{P}_{X}(Y)$ is a cyclic class. In particular, if the system $X$ is extensional and intensional, then the class $\mathcal{P}_{X}(X)$ of all predicative elements of $X$ is cyclic.

Corollary 10. Let $X$ be an arbitrary B-system.

(a) If $X$ satisfies the mixing principle, then $X$ satisfies the maximum principle (see [9] (1.10), [5] (6.1.7)). 
(b) If $X$ is intensional and satisfies the maximum principle for the formula $(\exists x)(x \in y)$, then $X$ satisfies the mixing principle (see [9] (1.12), [5] (6.1.9)).

(c) If $X$ is extensional and satisfies the ascent principle, then $X$ satisfies the mixing and maximum principles (see [9] (1.11), [5] (6.1.8)).

Proof. Item (a) is a consequence of Proposition 12 and Theorem 7; (b) follows from Corollary 6; and (c) follows from Corollary 9 and (a).

\subsection{Transitive Subsystems}

A set or a class $Y$ is called transitive if

$$
(\forall x, y)(x \in y \in Y \Rightarrow x \in Y)
$$

Transitive classes are traditionally used in set theory as models of fragments and modifications of set theory itself. Lévy's Lemma on the absoluteness of bounded formulas is a useful tool in working with such models. In this subsection, we study the notion of transitive Boolean-valued subsystem and prove the corresponding analog of Lévy's Lemma.

Throughout the sequel, $B$ is an arbitrary complete Boolean algebra.

Remark 16. If $Y$ is a subsystem of a B-system $X$ (see Definition 16) then the notation ${ }^{\%} Y$ can be understood in two ways: as the set $(Y \times B) / \sim$ of partial elements of the system $Y$ (see Definition 23) or as the subclass $\left\{\left.x\right|_{b}: x \in Y, b \in B\right\} \subset{ }^{\%} X$. The choice is immaterial in this case since the correspondence

$$
\sim_{Y}(y, b) \mapsto \sim_{X}(y, b), \quad y \in Y, b \in B,
$$

is a natural embedding of ${ }^{\%} Y=(Y \times B) / \sim$ into ${ }^{\%} X=(X \times B) / \sim$, and we may always assume that ${ }^{\%} Y \subset{ }^{\%} X$.

An analogous ambiguity appears in interpreting the symbol $P \uparrow$ for $P \subset{ }^{\%} Y$ (see Definition 28). The domain of the Boolean-valued class $P \uparrow$ depends on the system under consideration: $P \uparrow_{X}: X \rightarrow B$, $P \uparrow_{Y}: Y \rightarrow B$. Nevertheless, the choice of an interpretation is again immaterial, since $P \uparrow_{X}$ and $P \uparrow_{Y}$ coincide on $Y$; therefore, the truth value $[y \in P \uparrow]$ for $y \in Y$ does not depend on the system in which it is calculated. Indeed, if $y \in Y$ and $P=\left\{\left.y_{i}\right|_{b_{i}}: i \in I\right\}$, where $y_{i} \in Y$ and $b_{i} \in B$, then

$$
\begin{aligned}
& {\left[y \in P \uparrow_{Y}\right]_{Y}=P \uparrow_{Y}(y)=\bigvee_{i \in I}\left[y=\left.y_{i}\right|_{b_{i}}\right]_{Y}=\bigvee_{i \in I}\left[y=y_{i}\right]_{Y} \wedge b_{i} } \\
= & \bigvee_{i \in I}\left[y=y_{i}\right]_{X} \wedge b_{i}=\bigvee_{i \in I}\left[y=\left.y_{i}\right|_{b_{i}}\right]_{X}=P \uparrow_{X}(y)=\left[y \in P \uparrow_{X}\right]_{X} .
\end{aligned}
$$

For this reason, we may simply write $[y \in P \uparrow]$ instead of $\left[y \in P \uparrow_{Y}\right]_{Y}$ or $\left[y \in P \uparrow_{X}\right]_{X}$ and add the indices $X$ and $Y$ just to specify the system where the calculation is carried out.

Slightly more accuracy is needed in dealing with the formula $y \simeq P \uparrow$ for $y \in Y$ and $P \subset{ }^{\%} Y$ (see Definition 29). The relation $y \simeq P \uparrow_{X}$ in $X$ implies the analogous relation $y \simeq P \uparrow_{Y}$ in $Y$ but, as is shown in Proposition 18, the converse holds for all $y \in Y$ and $P \subset \% Y$ if and only if the subsystem $Y \subset X$ is transitive.

Definition 44. Let $X$ be an arbitrary B-system. Call a subclass $Y \subset X$ transitive in $X$ if the ascent $Y \uparrow$ is transitive inside $X$ :

$$
X \vDash(\forall x, y)(x \in y \in Y \uparrow \Rightarrow x \in Y \uparrow) .
$$

We say that $Y$ is a transitive subsystem of $X$ and write $Y \preccurlyeq X$ if $Y$ is a nonempty transitive subclass of $X$ endowed with the interpretations induced from $X$ (see Definition 16).

Proposition 17. The following properties of a subclass $Y \subset X$ are equivalent:

(a) $Y$ is transitive in $X$;

(b) $(\forall y \in Y) X \vDash(y \subset Y \uparrow)$; 
(c) $(\forall x \in X)(\forall y \in Y)[x \in y] \leqslant[x \in Y \uparrow]$;

(d) $(\forall x \in X)(\forall y \in Y)[x \in y]=\bigvee_{z \in Y}[x=z] \wedge[z \in y]$.

Proof. The implications $(\mathrm{a}) \Rightarrow(\mathrm{b}) \Leftrightarrow(\mathrm{c}) \Leftarrow(\mathrm{d})$ are obvious; $(\mathrm{b}) \Rightarrow(\mathrm{a})$ follows from Corollary 2 . (c) $\Rightarrow$ (d): By (c) and Proposition 7, if $x \in X$ and $y \in Y$ then

$$
\begin{gathered}
\bigvee_{z \in Y}[x=z] \wedge[z \in y] \leqslant[x \in y] \leqslant[x \in Y \uparrow] \wedge[x \in y] \\
\quad=\bigvee_{z \in Y}[x=z] \wedge[x \in y]=\bigvee_{z \in Y}[x=z] \wedge[z \in y] .
\end{gathered}
$$

Proposition 18. Let $Y$ be a subsystem of a B-system $X$. The following are equivalent:

(a) $X \vDash\left(y=P \uparrow_{X}\right) \Leftrightarrow Y \vDash\left(y=P \uparrow_{Y}\right)$ for every $y \in Y$ and an arbitrary class $P \subset{ }^{\%} Y$;

(b) $X \vDash\left(y=y \Downarrow_{Y} \uparrow_{X}\right)$ for all $y \in Y$;

(c) $Y \preccurlyeq X$.

Proof. $(a) \Rightarrow(b)$ : Owing to Lemma 7 , if $y \in Y$ then $Y \vDash\left(y=y \Downarrow_{Y} \uparrow_{Y}\right)$, and so $X \vDash\left(y=y \Downarrow_{Y} \uparrow_{X}\right)$ due to (a).

The implication $(b) \Rightarrow(c)$ is a consequence of Proposition $17(b)$ and Lemma $5(b)$.

(c) $\Rightarrow(a)$ : Let $Y \preccurlyeq X, y \in Y$, and $P \subset{ }^{\%} Y$. From Proposition 17 (b) and Lemma 5(b) it follows that $X \vDash\left(y \subset Y \uparrow_{X}, P \uparrow_{X} \subset Y \uparrow_{X}\right)$, and so

$$
X \vDash\left(y=y \cap Y \uparrow_{X}, P \uparrow_{X}=P \uparrow_{X} \cap Y \uparrow_{X}\right) .
$$

Therefore, from Corollary 2 we deduce that

$$
\begin{aligned}
X \vDash\left(y=P \uparrow_{X}\right) & \Leftrightarrow X \vDash\left(y \cap Y \uparrow_{X}=P \uparrow_{X} \cap Y \uparrow_{X}\right) \\
& \Leftrightarrow X \vDash\left(\forall z \in Y \uparrow_{X}\right)\left(z \in y \Leftrightarrow z \in P \uparrow_{X}\right) \\
& \Leftrightarrow(\forall z \in Y) X \vDash\left(z \in y \Leftrightarrow z \in P \uparrow_{X}\right) \\
& \Leftrightarrow(\forall z \in Y) Y \vDash\left(z \in y \Leftrightarrow z \in P \uparrow_{Y}\right) \\
& \Leftrightarrow Y \vDash(\forall z)\left(z \in y \Leftrightarrow z \in P \uparrow_{Y}\right) \\
& \Leftrightarrow Y \vDash\left(y=P \uparrow_{Y}\right) . \quad \square
\end{aligned}
$$

Definition 45. Let $\varphi$ be a formula of signature $\{=, \in\}$, and let $V$ be a variable or a class. The relativization of $\varphi$ to $V$ is the formula obtained from $\varphi$ by replacing each quantifier $(\forall x)$ and $(\exists x)$, where $x$ is an arbitrary variable, by the corresponding quantifier $(\forall x \in V)$ and $(\exists x \in V)$; see [2] (12.6). The relativization of $\varphi$ to $V$ is denoted by $V \vDash \varphi$ or, in more detail, $V \vDash \varphi(\ddot{x})$, where $\ddot{x}=x_{1}, \ldots, x_{n}$ is a list containing the parameters of $\varphi$. The notation for relativization stems from the fact that, for every $\ddot{x} \in V$, the assertion $V \vDash \varphi(\ddot{x})$ is equivalent to the validity of $\varphi(\ddot{x})$ in the two-valued algebraic system $\left(V,[=]_{V},[\in]_{V}\right)$ with the standard interpretation of equality and containment.

Definition 46. A formula is said to be bounded if each occurrence of a quantifier in it has the form $(\exists x \in y)$ or $(\forall x \in y)$. As an example of a bounded formula serves the relativization $V \vDash \varphi$ of any formula $\varphi$ to a variable $V$.

The following assertion is a consequence of the classical lemma by A. Lévy on the absoluteness of bounded formulas for transitive models; see [10] (Lemma 34), [2] (Lemma 12.9).

Lemma 23. Let $\varphi$ be a bounded formula with parameters $\ddot{y}$. If $X$ is a B-system and $Y \preccurlyeq X$ then

$$
[\varphi(\ddot{y})]_{X}=[\varphi(\ddot{y})]_{Y} \quad \text { for all } \ddot{y} \in Y .
$$


Proof. Since Lévy's Lemma is proven within the predicate calculus of signature $\{=, \in\}$ without special axioms; by Proposition 11, the conclusion of the lemma is valid in $X$ for the transitive Boolean-valued class $Y \uparrow_{X}$, that is,

$$
X \vDash\left(\forall \ddot{y} \in Y \uparrow_{X}\right)\left(\varphi(\ddot{y}) \Leftrightarrow Y \uparrow_{X} \vDash \varphi(\ddot{y})\right),
$$

where $Y \uparrow_{X} \vDash \varphi(\ddot{y})$ is the relativization of $\varphi(\ddot{y})$ to $Y \uparrow_{X}$. In particular, $[\varphi(\ddot{y})]_{X}=\left[Y \uparrow_{X} \vDash \varphi(\ddot{y})\right]_{X}$ for all $\ddot{y} \in Y$. The equality $\left[Y \uparrow_{X} \vDash \varphi(\ddot{y})\right]_{X}=[\varphi(\ddot{y})]_{Y}$ is easily proven by induction on the complexity of a bounded formula $\varphi$.

In what follows, we will need a stronger version of Lévy's Lemma that involves Boolean-valued classes.

Theorem 8. Let $\varphi$ be a bounded formula. Consider an arbitrary partition of the parameters of $\varphi$ into two parts, $\ddot{y}=y_{1}, \ldots, y_{m}$ and $\ddot{z}=z_{1}, \ldots, z_{n}$. If $X$ is a $B$-system and $Y \preccurlyeq X$ then

$$
[\varphi(\ddot{y}, \ddot{\Phi})]_{X}=\left[\varphi\left(\ddot{y},\left.\ddot{\Phi}\right|_{Y}\right)\right]_{Y} \text { for all } \ddot{y} \in Y \text { and } \ddot{\Phi} \Subset X, \ddot{\Phi} \leqslant Y \uparrow_{X^{\prime}}
$$

where $\ddot{\Phi}=\Phi_{1}, \ldots, \Phi_{n}$ and $\left.\ddot{\Phi}\right|_{Y}=\left.\Phi_{1}\right|_{Y}, \ldots,\left.\Phi_{n}\right|_{\gamma}$.

Proof. Refer to $\varphi$ as an absolute formula if ZFC proves (19) for every partition of the parameters into two parts. (Note that, owing to the presence of arbitrary Boolean-valued classes, (19) is an infinite assertion; see Section 2.4.) To justify the absoluteness of each bounded formula, we use the induction on the complexity of the formula.

Show that the atomic formulas are absolute. Assume that $y \in Y, \Phi, \Psi \Subset X$, and $\Phi, \Psi \leqslant Y \uparrow_{X}$. Then $X \vDash\left(y \subset Y \uparrow_{X}\right)$ due to the transitivity of the subsystem $Y$. Moreover, $X \vDash\left(\Phi, \Psi \subset Y \uparrow_{X}\right)$ by Lemma 5 (a). Therefore, using Corollary 2, we have

$$
\begin{aligned}
& {[y \in \Phi]_{X}=\Phi(y)=\left.\Phi\right|_{Y}(y)=\left[\left.y \in \Phi\right|_{Y}\right]_{Y} ;} \\
& {[y=\Phi]_{X}=[(\forall z)(z \in y \Leftrightarrow z \in \Phi)]_{X}=\left[\left(\forall z \in Y \uparrow_{X}\right)(z \in y \Leftrightarrow z \in \Phi)\right]_{X}} \\
& =\bigwedge_{z \in Y}[z \in y]_{X} \Leftrightarrow_{B}[z \in \Phi]_{X}=\bigwedge_{z \in Y}[z \in y]_{Y} \Leftrightarrow_{B}\left[\left.z \in \Phi\right|_{Y}\right]_{Y} \\
& =\left[(\forall z)\left(\left.z \in y \Leftrightarrow z \in \Phi\right|_{Y}\right)\right]_{Y}=\left[y=\left.\Phi\right|_{Y}\right]_{Y} ; \\
& {[\Phi \in y]_{X}=[(\exists z)(z=\Phi \wedge z \in y)]_{X}=\left[\left(\exists z \in Y \uparrow_{X}\right)(z=\Phi \wedge z \in y)\right]_{X}} \\
& =\bigvee_{z \in Y}[z=\Phi]_{X} \wedge[z \in y]_{X}=\bigvee_{z \in Y}\left[z=\left.\Phi\right|_{Y}\right]_{Y} \wedge[z \in y]_{Y} \\
& =\left[(\exists z)\left(z=\left.\Phi\right|_{Y} \wedge z \in y\right)\right]_{Y}=\left[\left.\Phi\right|_{Y} \in y\right]_{Y} \text {; } \\
& {[\Phi=\Psi]_{X}=[(\forall z)(z \in \Phi \Leftrightarrow z \in \Psi)]_{X}=\left[\left(\forall z \in Y \uparrow_{X}\right)(z \in \Phi \Leftrightarrow z \in \Psi)\right]_{X}} \\
& =\bigwedge_{z \in Y}[z \in \Phi]_{X} \Leftrightarrow_{B}[z \in \Psi]_{X}=\bigwedge_{z \in Y}\left[\left.y \in \Phi\right|_{Y}\right]_{Y} \Leftrightarrow_{B}\left[\left.z \in \Psi\right|_{Y}\right]_{Y} \\
& =\left[(\forall z)\left(\left.\left.z \in \Phi\right|_{Y} \Leftrightarrow z \in \Psi\right|_{Y}\right)\right]_{Y}=\left[\left.\Phi\right|_{Y}=\left.\Psi\right|_{Y}\right]_{Y} ; \\
& {[\Phi \in \Psi]_{X}=[(\exists z)(z=\Phi \wedge z \in \Psi)]_{X}=\left[\left(\exists z \in Y \uparrow_{X}\right)(z=\Phi \wedge z \in \Psi)\right]_{X}} \\
& =\bigvee_{z \in Y}[z=\Phi]_{X} \wedge[z \in \Psi]_{X}=\bigvee_{z \in Y}\left[z=\left.\Phi\right|_{Y}\right]_{Y} \wedge\left[\left.z \in \Psi\right|_{Y}\right]_{Y} \\
& =\left[(\exists z)\left(z=\left.\left.\Phi\right|_{Y} \wedge z \in \Psi\right|_{Y}\right)\right]_{Y}=\left[\left.\left.\Phi\right|_{Y} \in \Psi\right|_{Y}\right]_{Y} .
\end{aligned}
$$

As is easy to see, the absoluteness of formulas is preserved by negation and disjunction. It remains to show that the absoluteness of $\varphi(x, y, \ddot{y}, \ddot{z})$ implies the absoluteness of 
$\psi(y, \ddot{y}, \ddot{z}):=(\exists x \in y) \varphi(x, y, \ddot{y}, \ddot{z})$. Indeed, if $y, \ddot{y} \in Y, \Phi, \ddot{\Phi} \Subset X$, and $\Phi, \ddot{\Phi} \leqslant Y \uparrow_{X}$; then, in view of the relations $X \vDash\left(y \subset Y \uparrow_{X}\right), X \vDash\left(\Phi \subset Y \uparrow_{X}\right)$ and the absoluteness of $\varphi$, we have

$$
\begin{aligned}
{[\psi(y, \ddot{y}, \ddot{\Phi})]_{X} } & =[(\exists x)(x \in y \wedge \varphi(x, y, \ddot{y}, \ddot{\Phi}))]_{X}=\left[\left(\exists x \in Y \uparrow_{X}\right)(x \in y \wedge \varphi(x, y, \ddot{y}, \ddot{\Phi}))\right]_{X} \\
& =\bigvee_{x \in Y}[x \in y]_{X} \wedge[\varphi(x, y, \ddot{y}, \ddot{\Phi})]_{X}=\bigvee_{x \in Y}[x \in y]_{Y} \wedge\left[\varphi\left(x, y, \ddot{y},\left.\ddot{\Phi}\right|_{Y}\right)\right]_{Y} \\
& =\left[(\exists x)\left(x \in y \wedge \varphi\left(x, y, \ddot{y},\left.\ddot{\Phi}\right|_{Y}\right)\right)\right]_{Y}=\left[\psi\left(y, \ddot{y},\left.\ddot{\Phi}\right|_{Y}\right)\right]_{Y} ; \\
{[\psi(\Phi, \ddot{y}, \ddot{\Phi})]_{X} } & =[(\exists x)(x \in \Phi \wedge \varphi(x, \Phi, \ddot{y}, \ddot{\Phi}))]_{X}=\left[\left(\exists x \in Y \uparrow_{X}\right)(x \in \Phi \wedge \varphi(x, \Phi, \ddot{y}, \ddot{\Phi}))\right]_{X} \\
& =\bigvee_{x \in Y}[x \in \Phi]_{X} \wedge[\varphi(x, \Phi, \ddot{y}, \ddot{\Phi})]_{X}=\bigvee_{x \in Y}\left[\left.x \in \Phi\right|_{Y}\right]_{Y} \wedge\left[\varphi\left(x,\left.\Phi\right|_{Y}, \ddot{y},\left.\ddot{\Phi}\right|_{Y}\right)\right]_{Y} \\
& =\left[(\exists x)\left(\left.x \in \Phi\right|_{Y} \wedge \varphi\left(x,\left.\Phi\right|_{Y}, \ddot{y},\left.\ddot{\Phi}\right|_{Y}\right)\right)\right]_{Y}=\left[\psi\left(\left.\Phi\right|_{Y}, \ddot{y},\left.\ddot{\Phi}\right|_{Y}\right)\right]_{Y} .
\end{aligned}
$$

The following is an analog of Lévy's Lemma for formulas with quantifiers over predicative Boolean-valued classes:

Lemma 24. Let $\varphi$ be a bounded formula. If $X$ is a B-system and $Y \preccurlyeq X$ then

$$
[(\mathbb{\forall} \Phi \subset \Psi) \varphi(\Phi, \ddot{y}, \ddot{\Psi})]_{X}=\left[\left(\left.\mathbb{\forall} \Phi \subset \Psi\right|_{Y}\right) \varphi\left(\Phi, \ddot{y},\left.\dddot{\Psi}\right|_{Y}\right)\right]_{Y}
$$

for all $\ddot{y} \in Y$ and $\Psi, \ddot{\Psi} \Subset X, \Psi, \ddot{\Psi} \leqslant Y \uparrow_{X}$.

Proof. If $\Phi \Subset Y$ and $\Phi \leqslant\left.\Psi\right|_{Y}$ then $\Phi \uparrow_{X} \leqslant \Psi$ by Lemma $3(b)$ and, moreover, $\left.\left(\Phi \uparrow_{X}\right)\right|_{Y}=\Phi$. Therefore, using Theorem 8 and Lemma 9, we have

$$
\begin{gathered}
{[(\mathbb{\forall} \Phi \subset \Psi) \varphi(\Phi, \ddot{y}, \ddot{\Psi})]_{X}=\bigwedge_{\Phi \Subset X: \Phi \leqslant \Psi}[\varphi(\Phi, \ddot{y}, \ddot{\Psi})]_{X}} \\
\leqslant \bigwedge_{\Phi \Subset Y: \Phi \leqslant\left.\Psi\right|_{Y}}\left[\varphi\left(\Phi \uparrow_{X}, \ddot{y}, \ddot{\Psi}\right)\right]_{X}=\bigwedge_{\Phi \Subset Y: \Phi \leqslant\left.\Psi\right|_{Y}}\left[\varphi\left(\left.\left(\Phi \uparrow_{X}\right)\right|_{Y}, \ddot{y},\left.\ddot{\Psi}\right|_{Y}\right)\right]_{Y} \\
=\bigwedge_{\Phi \Subset Y: \Phi \leqslant\left.\Psi\right|_{Y}}\left[\varphi\left(\Phi, \ddot{y},\left.\ddot{\Psi}\right|_{Y}\right)\right]_{Y}=\left[\left(\left.\mathbb{\Psi} \Phi \subset \Psi\right|_{Y}\right) \varphi\left(\Phi, \ddot{y},\left.\ddot{\Psi}\right|_{Y}\right)\right]_{Y} .
\end{gathered}
$$

The reverse inequality is also guaranteed by Theorem 8 and Lemma 9:

$$
\begin{aligned}
& {\left[\left(\left.\mathbb{\forall} \Phi \subset \Psi\right|_{Y}\right) \varphi\left(\Phi, \ddot{y},\left.\ddot{\Psi}\right|_{Y}\right)\right]_{Y}=\bigwedge_{\Phi \Subset Y: \Phi \leqslant\left.\Psi\right|_{Y}}\left[\varphi\left(\Phi, \ddot{y},\left.\ddot{\Psi}\right|_{Y}\right)\right]_{Y} } \\
\leqslant & \bigwedge_{\Phi \Subset X:\left.\Phi\right|_{Y} \leqslant\left.\Psi\right|_{Y}}\left[\varphi\left(\left.\Phi\right|_{Y}, \ddot{y},\left.\ddot{\Psi}\right|_{Y}\right)\right]_{Y}=\bigwedge_{\Phi \Subset X:\left.\Phi\right|_{Y} \leqslant\left.\Psi\right|_{Y}}[\varphi(\Phi, \ddot{y}, \ddot{\Psi})]_{X} \\
\leqslant & \bigwedge_{\Phi \Subset X: \Phi \leqslant \Psi}[\varphi(\Phi, \ddot{y}, \ddot{\Psi})]_{X}=[(\mathbb{V} \Phi \Psi) \varphi(\Phi, \ddot{y}, \ddot{\Psi})]_{X} .
\end{aligned}
$$

Corollary 11. If a B-system $X$ is extensional and $Y \preccurlyeq X$ then $Y$ is extensional.

Proof. By Definition 27, the extensionality of $X$ means that $X \vDash(\forall x, y) \varphi(x, y)$, where

$$
\varphi(x, y):=(\forall z \in x)(z \in y) \wedge(\forall z \in y)(z \in x) \Rightarrow x=y .
$$

Thus, if $X$ is extensional then $X \vDash\left(\forall x, y \in Y \uparrow_{X}\right) \varphi(x, y)$, whence, by Theorem 8 and the boundedness of the formula $(\forall x, y \in u) \varphi(x, y)$, we have $Y \vDash\left(\forall x,\left.y \in\left(Y \uparrow_{X}\right)\right|_{Y}\right) \varphi(x, y)$, that is, $Y \vDash(\forall x, y) \varphi(x, y)$, which is equivalent to the extensionality of $Y$.

Corollary 12. Let $\varphi(\ddot{y}, \ddot{z})$ be an arbitrary formula. If $X$ is a $B$-system and $Y \preccurlyeq X$ then

$$
\left[Y \uparrow_{X} \vDash \varphi(\ddot{y}, \ddot{\Phi})\right]_{X}=\left[\varphi\left(\ddot{y},\left.\ddot{\Phi}\right|_{Y}\right)\right]_{Y}
$$


for all $\ddot{y} \in Y$ and $\ddot{\Phi} \Subset X, \ddot{\Phi} \leqslant Y \uparrow_{X}$, where $x \vDash \varphi$ is the relativization of $\varphi$ to $x$.

Proof. Since the formula $x \vDash \varphi$ is bounded, Theorem 8 implies

$$
\left[Y \uparrow_{X} \vDash \varphi(\ddot{y}, \ddot{\Phi})\right]_{X}=\left[\left.\left(Y \uparrow_{X}\right)\right|_{Y} \vDash \varphi\left(\ddot{y},\left.\ddot{\Phi}\right|_{Y}\right)\right]_{Y}=\left[Y \uparrow_{Y} \vDash \varphi\left(\ddot{y},\left.\ddot{\Phi}\right|_{Y}\right)\right]_{Y}=\left[\varphi\left(\ddot{y},\left.\ddot{\Phi}\right|_{Y}\right)\right]_{Y} .
$$

Agreement 8. Let I be a nonempty set or class. Call a family of B-systems $\left(X_{i}\right)_{i \in I}$ directed if for all $i, j \in I$ there is $k \in I$ such that $X_{i}$ and $X_{j}$ are subsystems of $X_{k}$. As is easy to see, on the union $X:=\bigcup_{i \in I} X_{i}$ of such a family, there is a unique pair of functions $[=]_{X},[\in]_{X}: X^{2} \rightarrow B$ turning $X$ into a B-system that includes all the systems $X_{i}$ as subsystems. With this circumstance in mind, when considering a directed family of B-systems, agree to tacitly assume the union of the family to be a B-system.

\section{Proposition 19.}

(a) Let $Y$ and $Z$ be subsystems of a $B$-system $X$. If $Z \preccurlyeq X$ and $Z \subset Y$ then $Z \preccurlyeq Y$.

(b) If $X, Y$, and $Z$ are $B$-systems and $Z \preccurlyeq Y \preccurlyeq X$ then $Z \preccurlyeq X$.

(c) Let $\left(X_{i}\right)_{i \in I}$ be a nonempty family of subsystems of a B-system $X$. If $X_{i} \preccurlyeq X$ for all $i \in I$ then $\bigcup_{i \in I} X_{i} \preccurlyeq X$.

(d) Let I be a nonempty directed ordered set or class, let $\left(X_{i}\right)_{i \in I}$ be a family of B-systems, and let $X_{i} \preccurlyeq X_{j}$ for $i \leqslant j$. Then $X_{i} \preccurlyeq \bigcup_{j \in I} X_{j}$ for all $i \in I$.

(e) Suppose that $\left(X_{\alpha}\right)_{\alpha \in \text { Ord }}$ is a family of B-systems, $X_{\alpha} \preccurlyeq X_{\alpha+1}$ for $\alpha \in$ Ord, and $\bigcup_{\beta<\alpha} X_{\beta} \preccurlyeq X_{\alpha}$ for $\alpha \in \operatorname{Lim}$ Ord. Then $X_{\gamma} \preccurlyeq X_{\beta} \preccurlyeq \bigcup_{\alpha \in \text { Ord }} X_{\alpha}$ for all $\gamma \leqslant \beta \in$ Ord.

Proof. (a): If $Z \subset Y \subset X, Z \preccurlyeq X, y \in Y$, and $z \in Z$, then

$$
[y \in z] \leqslant\left[y \in Z \uparrow_{X}\right]_{X}=\left[y \in Z \uparrow_{Y}\right]_{Y} .
$$

(b): If $Z \preccurlyeq Y \preccurlyeq X$ then, using Proposition 17 (d), for all $x \in X$ and $z \in Z$, we have

$$
[x \in z]=\bigvee_{y \in Y}[x=y] \wedge[y \in z] \leqslant \bigvee_{y \in Y}[x=y] \wedge[y \in Z \uparrow] \leqslant[x \in Z \uparrow]
$$

(c): If $x \in X$ and $y \in Y:=\bigcup_{i \in I} X_{i}$ then $y \in X_{i}$ for some $i \in I$. Since $X_{i} \preccurlyeq X$, this implies $[x \in y] \leqslant\left[x \in X_{i} \uparrow\right]_{X} \leqslant[x \in Y \uparrow]_{X}$.

(d): If $i \in I, x \in X:=\bigcup_{j \in I} X_{j}$, and $y \in X_{i}$; then $x \in X_{j}$ for some $j \in I, i \leqslant j$. Since $X_{i} \preccurlyeq X_{j}$, it follows that $[x \in y] \leqslant\left[x \in X_{i} \uparrow\right]_{X_{j}}=\left[x \in X_{i} \uparrow\right]_{X}$.

(e): Given $0 \neq \alpha \in$ Ord, put $\mathcal{X}_{\alpha}:=\bigcup_{\beta<\alpha} X_{\beta}$ and observe that $\mathcal{X}_{\alpha} \preccurlyeq X_{\alpha}$. Indeed, for $\alpha \in$ Lim Ord, this relation explicitly occurs in the hypothesis; and if $\alpha=\alpha_{0}+1$ then, by the obvious monotonicity of the family $\left(X_{\alpha}\right)_{\alpha \in \text { Ord }}$, we have

$$
\mathcal{X}_{\alpha}=\bigcup_{\beta<\alpha} X_{\beta}=\bigcup_{\beta \leqslant \alpha_{0}} X_{\beta}=X_{\alpha_{0}} \preccurlyeq X_{\alpha_{0}+1}=X_{\alpha} .
$$

Show by induction on $\alpha$ that $X_{\beta} \preccurlyeq X_{\alpha}$ for $\beta<\alpha$. Consider an arbitrary ordinal $\alpha$, suppose that

$$
X_{\gamma} \preccurlyeq X_{\beta} \text { for } \gamma<\beta<\alpha,
$$

and establish the relation $X_{\beta} \preccurlyeq X_{\alpha}$ for all $\beta<\alpha$. If $\beta<\alpha$ then, by (20) and (d), we have $X_{\beta} \preccurlyeq \mathcal{X}_{\alpha}$, whence, owing to $\mathcal{X}_{\alpha} \preccurlyeq X_{\alpha}$ and using (b), we have $X_{\beta} \preccurlyeq X_{\alpha}$.

What was said above, together with (d), implies $X_{\beta} \preccurlyeq \bigcup_{\alpha \in \text { Ord }} X_{\alpha}$ for all $\beta \in$ Ord.

\subsection{Regular Systems}

In the present subsection, we introduce and study regular Boolean-valued systems. Those are the systems in which the axiom of regularity is valid for arbitrary predicative Boolean-valued classes. 
Definition 47. The axiom of regularity (foundation) has the form $(\forall x) \mu(x)$, where

$$
\mu(x):=((\exists y)(y \in x) \Rightarrow(\exists y \in x)(\forall z \in x)(z \notin y))
$$

or, which is the same,

$$
\mu(x):=(x \neq \varnothing \Rightarrow(\exists y \in x)(y \cap x=\varnothing)) .
$$

(The name for $\mu(x)$ is chosen to suggest that $x$, if nonempty, has an $\in$-minimal element.) If $\mathbb{V}$ is the class of all sets and $\left(\mathbb{V}_{\alpha}\right)_{\alpha \in \text { Ord }}$ is the von Neumann cumulative hierarchy defined by the recursive rule

$$
\begin{aligned}
& \mathbb{V}_{0}=\varnothing ; \\
& \mathbb{V}_{\alpha+1}=\mathcal{P}\left(\mathbb{V}_{\alpha}\right), \quad \alpha \in \text { Ord; } \\
& \mathbb{V}_{\alpha}=\bigcup_{\beta<\alpha} \mathbb{V}_{\beta}, \quad \alpha \in \text { Lim Ord; }
\end{aligned}
$$

then the equality $\mathbb{V}=\bigcup_{\alpha \in \text { Ord }} \mathbb{V}_{\alpha}$ is equivalent to the axiom of regularity in the theory obtained from ZFC by excluding the axiom; see [2] (Section 6). Moreover, in this theory, the axiom of regularity is equivalent to $\sigma$-regularity (see Section 3.9 below), that is the absence of a sequence $\left(x_{n}\right)_{n \in \mathbb{N}}$ with $x_{n+1} \in x_{n}$ for all $n \in \mathbb{N}$.

Definition 48. Let $X$ be a B-system and let $\Psi \Subset X$. Say that the Boolean-valued class $\Psi$ is regular in $X$ if

$$
X \vDash(\mathbb{\forall} \Phi \subset \Psi) \mu(\Phi)
$$

(see (21)). Say that the B-system $X$ is regular outside a subclass $Y \subset X$ if the complement $\neg(Y \uparrow)$ of the Boolean-valued class $Y \uparrow$ is regular in $X$ :

$$
X \vDash(\mathbb{\forall} \Phi)(\Phi \cap Y \uparrow=\varnothing \Rightarrow \mu(\Phi)) .
$$

Call the system X regular if the greatest Boolean-valued class $X \uparrow$ is regular in X:

$$
X \vDash(\mathbb{\nabla}) \mu(\Phi)
$$

The regularity of $X$ is obviously related to the validity of the axiom of regularity $(\forall x) \mu(x)$ in $X:$

\section{Proposition 20.}

(a) If $X$ is predicative then the regularity of $X$ implies the validity $X \vDash(\forall x) \mu(x)$.

(b) If $X$ is intensional then the validity $X \vDash(\forall x) \mu(x)$ implies the regularity of $X$.

(c) If $X$ satisfies the ascent principle then the regularity of $X$ is equivalent to the validity $X \vDash(\forall x) \mu(x)$.

Lemma 25. The following properties of a B-system $X$ are equivalent:

(a) $X$ is not regular;

(b) there exists a set $P \subset{ }^{\%} X$ such that

$$
\begin{aligned}
& (\exists p \in P) \Delta p \neq 0_{B}, \\
& (\forall p \in P) \bigvee_{q \in P}[q \in p] \geqslant \Delta p ;
\end{aligned}
$$


(c) there exists a sequence of sets $P_{n} \subset{ }^{\%} X(n \in \mathbb{N})$ such that

$$
\begin{aligned}
& P_{n} \text { is an antichain, } \\
& \bigvee_{p \in P_{n}} \Delta p=\bigvee_{p \in P_{1}} \Delta p \neq 0_{B}, \\
& \left(\forall p \in P_{n}\right)\left(\forall q \in P_{n+1}\right)\left(\Delta p \wedge \Delta q \neq 0_{B} \Rightarrow \Delta q=[q \in p]\right), \\
& \left(\forall p \in P_{n}\right) \bigvee_{q \in P_{n+1}}[q \in p]=\Delta p .
\end{aligned}
$$

Proof. (a) $\Rightarrow$ (b): If $X$ is not regular; then, by Theorems 4 (a) and 5 , there exists a set $P \subset{ }^{\%} X$ for which $X \not \models \mu(P \uparrow)$. The latter means that

$$
b:=[P \uparrow \neq \varnothing] \wedge[(\forall y \in P \uparrow)(\exists z \in P \uparrow)(z \in y)] \neq 0_{B} .
$$

Show that the set $\left\{\left.p\right|_{b}: p \in P\right\}$ satisfies (b). Indeed, with account taken of Lemma 2(b),

$$
\left.\bigvee_{p \in P} \Delta p\right|_{b}=\bigvee_{p \in P} \Delta p \wedge b=[P \uparrow \neq \varnothing] \wedge b=b \neq 0_{B}
$$

Moreover, by Lemma 6,

$$
b \leqslant[(\forall y \in P \uparrow)(\exists z \in P \uparrow)(z \in y)]=\bigwedge_{p \in P} \neg \Delta p \vee \bigvee_{q \in P}[q \in p]
$$

Therefore, for all $p \in P$, we have $b \leqslant \neg \Delta p \vee \bigvee_{q \in P}[q \in p]$, and so

$$
\bigvee_{q \in P}\left[\left.q \in p\right|_{b}\right]=\bigvee_{q \in P}[q \in p] \wedge \Delta p \wedge b=\left(\neg \Delta p \vee \bigvee_{q \in P}[q \in p]\right) \wedge \Delta p \wedge b \geqslant \Delta p \wedge b=\left.\Delta p\right|_{b}
$$

(b) $\Rightarrow$ (c): Suppose that $P \subset{ }^{\%} X$ satisfies (b). By the exhaustion principle, for each $p \in{ }^{\%} P$, the equality $\bigvee_{q \in P}[q \in p]=\Delta p$ implies the existence of an antichain $D(p) \subset{ }^{\%} P$ such that $\Delta q=[q \in p]$ for all $q \in D(p)$ and $\bigvee_{q \in D(p)}[q \in p]=\Delta p$. For the same reason, there exists an antichain $P_{1} \subset{ }^{\%} P$ such that

$$
\bigvee_{p \in P_{1}} \Delta p=\bigvee_{p \in P} \Delta p \neq 0_{B}
$$

Define the sets $P_{n} \subset{ }^{\%} P(n \in \mathbb{N})$ by putting

$$
P_{n+1}:=\bigcup_{p \in P_{n}} D(p), \quad n \in \mathbb{N} .
$$

An elementary check shows that the sets $P_{n}$ satisfy all the conditions in (c).

(c) $\Rightarrow$ (a): Suppose that a sequence $\left(P_{n}\right)_{n \in \mathbb{N}}$ satisfies (c). Put $P:=\bigcup_{n \in \mathbb{N}} P_{n}$ and show that $X \not \models \mu(P \uparrow)$. Indeed, according to Lemma 2(b),

$$
[P \uparrow \neq \varnothing]=\bigvee_{p \in P} \Delta p \geqslant \bigvee_{p \in P_{1}} \Delta p \neq 0_{B}
$$

On the other hand, by Lemma 6 we have

$$
\begin{gathered}
{[(\exists y \in P \uparrow)(\forall z \in P \uparrow)(z \notin y)]=[(\exists y \in P \uparrow) \neg(\exists z \in P \uparrow)(z \in y)]} \\
=\bigvee_{p \in P} \Delta p \wedge \neg \bigvee_{q \in P} \bigvee_{y \in X: p \sqsubset y}[q \in y] \leqslant \bigvee_{p \in P} \Delta p \wedge \neg \bigvee_{q \in P}[q \in p]=\bigvee_{n \in \mathbb{N}} \bigvee_{p \in P_{n}} \Delta p \wedge \neg \bigvee_{q \in P}[q \in p] \\
\leqslant \bigvee_{n \in \mathbb{N}} \bigvee_{p \in P_{n}} \Delta p \wedge \neg \bigvee_{q \in P_{n+1}}[q \in p]=\bigvee_{n \in \mathbb{N}} \bigvee_{p \in P_{n}} \Delta p \wedge \neg \Delta p=0_{B} . \square
\end{gathered}
$$


The following is a consequence of Lemma 24 owing to the boundedness of $\mu(x)$ :

Corollary 13. Suppose that $X$ is a B-system, $Y \preccurlyeq X, \Psi \Subset X$, and $\Psi \leqslant Y \uparrow_{X}$. The class $\Psi$ is regular in $X$ if and only if the class $\left.\Psi\right|_{Y}$ is regular in $Y$.

Lemma 26. Let $X$ and $Y$ be $B$-systems and let $Z \subset Y \preccurlyeq X$. If $Y$ is regular outside $Z$ then the assertion on the regularity of the difference $Y \uparrow \backslash Z \uparrow$ is valid inside $X$ :

$$
X \vDash(\mathbb{\nabla} \subset Y \uparrow \backslash Z \uparrow) \mu(\Phi)
$$

Proof. Put $\Psi:=Y \uparrow_{X} \wedge \neg\left(Z \uparrow_{X}\right)$. The regularity of $Y$ outside $Z$ means that the class $\neg\left(Z \uparrow_{Y}\right)$ is regular in $Y$, which, in view of Corollary 13 and the relations $\Psi \leqslant Y \uparrow_{X},\left.\Psi\right|_{Y}=\neg\left(Z \uparrow_{Y}\right)$, implies the regularity of the class $\Psi$ in $X$. It remains to observe that $X \vDash(\Psi=Y \uparrow \backslash Z \uparrow)$.

Lemma 27. Suppose that $X$ and $Y$ are $B$-systems and $Z \subset Y \preccurlyeq X$. If $Y$ is regular outside $Z$ and $X$ is regular outside $Y$ then $X$ is regular outside $Z$.

Proof. Validate $X \vDash(\mathbb{\forall} \Phi)(\Phi \cap Z \uparrow=\varnothing \Rightarrow \mu(\Phi))$ by "reasoning inside $X^{\prime \prime}$ (see Remark 11). Let $\Phi \cap Z \uparrow=\varnothing$ and $\Phi \neq \varnothing$. The aim is to find $y \in \Phi$ such that $y \cap \Phi=\varnothing$. If $\Phi \cap Y \uparrow=\varnothing$ then a desired $y$ exists by the regularity of $\neg Y \uparrow$. Now, let $\Phi \cap Y \uparrow \neq \varnothing$. Since the class $Y \uparrow \backslash Z \uparrow$ is regular (see Lemma 26) and $\varnothing \neq \Phi \cap Y \uparrow \subset Y \uparrow \backslash Z \uparrow$, there exists $y \in \Phi \cap Y \uparrow$ such that $y \cap \Phi \cap Y \uparrow=\varnothing$. Then $y \cap \Phi=y \cap \Phi \cap Y \uparrow=\varnothing$ since, by the transitivity of $Y \uparrow$, from $y \in Y \uparrow$ it follows that $y \subset Y \uparrow$.

Corollary 14. Suppose that $X$ and $Y$ are B-systems and $Y \preccurlyeq X$. If $Y$ is regular and $X$ is regular outside $Y$ then $X$ is regular.

Proposition 21. If a B-system $X$ is regular then every subsystem $Y \subset X$ is regular.

Proof. Consider arbitrary families $\left(y_{i}\right)_{i \in I} \subset Y$ and $\left(b_{i}\right)_{i \in I} \subset B$ and show that $Y \vDash \mu\left(P \uparrow_{\gamma}\right)$, where $P:=\left\{\left.y_{i}\right|_{b_{i}}: i \in I\right\}$. Indeed, by Lemma 6 and the regularity of $X$, we conclude that

$$
\begin{gathered}
{\left[P \uparrow_{Y} \neq \varnothing\right]_{Y}=\bigvee_{i \in I} b_{i}=\left[P \uparrow_{X} \neq \varnothing\right]_{X} \leqslant\left[\left(\exists y \in P \uparrow_{X}\right)\left(\forall z \in P \uparrow_{X}\right)(z \notin y)\right]_{X}} \\
=\bigvee_{i \in I} \bigwedge_{j \in I}\left(\left[y_{j} \notin y_{i}\right]_{X} \wedge b_{i} \wedge b_{j}\right) \vee \neg b_{j}=\bigvee_{i \in I} \bigwedge_{j \in I}\left(\left[y_{j} \notin y_{i}\right]_{Y} \wedge b_{i} \wedge b_{j}\right) \vee \neg b_{j} \\
=\left[\left(\exists y \in P \uparrow_{Y}\right)\left(\forall z \in P \uparrow_{Y}\right)(z \notin y)\right]_{Y} .
\end{gathered}
$$

\subsection{Sequentially Regular Systems}

In this subsection, we introduce and study the notion of $\sigma$-regular Boolean-valued system and describe the complete Boolean algebras for which regularity and $\sigma$-regularity coincide.

Definition 49. Let $X$ be a B-system. Say that a class $\Psi \Subset X$ is $\sigma$-regular in $X$ if

$$
\bigwedge_{n \in \mathbb{N}}\left[x_{n} \in \Psi\right] \wedge\left[x_{n+1} \in x_{n}\right]=0_{B}
$$

for every sequence $\left(x_{n}\right)_{n \in \mathbb{N}} \subset X$. Say that $X$ is $\sigma$-regular outside a subclass $Y \subset X$ if the complement $\neg(Y \uparrow)$ to the Boolean-valued class $Y \uparrow$ is $\sigma$-regular in $X$; that is,

$$
\bigwedge_{n \in \mathbb{N}}\left[x_{n} \notin Y \uparrow\right] \wedge\left[x_{n+1} \in x_{n}\right]=0_{B}
$$


for every $\left(x_{n}\right)_{n \in \mathbb{N}} \subset X$. Call $X \sigma$-regular if the greatest class $X \uparrow$ is $\sigma$-regular in $X$ or, which is the same, $X$ is $\sigma$-regular outside $\varnothing$; that is,

$$
\bigwedge_{n \in \mathbb{N}}\left[x_{n+1} \in x_{n}\right]=0_{B}
$$

for every $\left(x_{n}\right)_{n \in \mathbb{N}} \subset X$.

Theorem 9. Let $X$ be a B-system.

(a) If a class $\Psi \Subset X$ is regular in $X$ then $\Psi$ is $\sigma$-regular in $X$.

(b) If $Y \subset X$ and the difference $X \backslash Y$ is precyclic then the regularity and the $\sigma$-regularity of $X$ outside $Y$ are equivalent.

(c) If $X$ satisfies the mixing principle then the regularity and the $\sigma$-regularity of $X$ are equivalent.

Proof. (a): Suppose that $\Psi$ is regular in $X$. Consider an arbitrary sequence $\left(x_{n}\right)_{n \in \mathbb{N}} \subset X$ and show that

$$
b:=\bigwedge_{n \in \mathbb{N}}\left[x_{n} \in \Psi\right] \wedge\left[x_{n+1} \in x_{n}\right]=0_{B} .
$$

Put $\Phi:=\left\{x_{n}: n \in \mathbb{N}\right\} \uparrow$. Then $[\Phi \neq \varnothing]=1_{B}$; and, moreover, by Corollary 2,

$$
[\Phi \subset \Psi]=[(\forall x \in \Phi)(x \in \Psi)]=\bigwedge_{n \in \mathbb{N}}\left[x_{n} \in \Psi\right] \geqslant b,
$$

which, in view of the regularity of $\Psi$ in $X$, implies that $[(\exists y \in \Phi)(\forall z \in \Phi)(z \notin y)] \geqslant b$.

On the other hand, from Corollary 2 we conclude that

$$
\begin{aligned}
& {[(\exists y \in \Phi)(\forall z \in \Phi)(z \notin y)]=\bigvee_{n \in \mathbb{N}} \bigwedge_{m \in \mathbb{N}}\left[x_{m} \notin x_{n}\right]} \\
& =\neg \bigwedge_{n \in \mathbb{N}} \bigvee_{m \in \mathbb{N}}\left[x_{m} \in x_{n}\right] \leqslant \neg \bigwedge_{n \in \mathbb{N}}\left[x_{n+1} \in x_{n}\right] \leqslant \neg b .
\end{aligned}
$$

(b): Suppose that the difference $Z:=X \backslash Y$ is nonempty and precyclic (see Corollary 7), and that $X$ is $\sigma$-regular outside $Y$. Consider an arbitrary class $\Phi \Subset X$ satisfying $\Phi \leqslant \neg(Y \uparrow)$ and show that $X \vDash \mu(\Phi)$; that is,

$$
b:=[\Phi \neq \varnothing \wedge(\forall y \in \Phi)(y \cap \Phi \neq \varnothing)]=0_{B} .
$$

Observe first that, for every Boolean-valued class $\Psi \Subset X$,

$$
(\exists x \in X)[(\exists y \in \Phi) \Psi(y)]=[x \in \Phi] \wedge[\Psi(x)] .
$$

Indeed, by Lemma 5(d),

$$
X \vDash(\Phi \subset X \uparrow \backslash Y \uparrow \subset Z \uparrow),
$$

which, by Corollary 2 and Lemma 16, implies the equalities

$$
\begin{gathered}
{[(\exists y \in \Phi) \Psi(y)]=[(\exists y \in Z \uparrow)(\Phi(y) \wedge \Psi(y))]} \\
=[(\exists y \in(\operatorname{mix} Z) \uparrow)(\Phi(y) \wedge \Psi(y))]=\bigvee_{y \in \operatorname{mix} Z} \Phi(y) \wedge \Psi(y) .
\end{gathered}
$$

Moreover, owing to Theorem 6, the function $\Phi \wedge \Psi$ attains its maximum on the cyclic class $\operatorname{mix} Z$.

By (23), there is an element $x_{1} \in X$ such that

$$
\left[x_{1} \in \Phi\right]=[\Phi \neq \varnothing] \geqslant b .
$$

Since $[(\forall y \in \Phi)(y \cap \Phi \neq \varnothing)] \geqslant b$, we have $\left[x_{1} \cap \Phi \neq \varnothing\right] \geqslant b$, that is, $\left[(\exists y \in \Phi)\left(y \in x_{1}\right)\right] \geqslant b$; and so, by (23), there exists $x_{2} \in X$, such that 


$$
\left[x_{2} \in \Phi\right] \wedge\left[x_{2} \in x_{1}\right] \geqslant b .
$$

"Iterating" these arguments (and, strictly speaking, applying recursion and the axiom of choice), we obtain a sequence $\left(x_{n}\right)_{n \in \mathbb{N}}$ of elements in $X$ satisfying

$$
\left[x_{n} \notin Y \uparrow\right] \wedge\left[x_{n+1} \in x_{n}\right] \geqslant\left[x_{n} \in \Phi\right] \wedge\left[x_{n+1} \in x_{n}\right] \geqslant b, \quad n \in \mathbb{N} .
$$

Owing to the $\sigma$-regularity of $X$ outside $Y$, this implies $b=0$.

Assertion (c) is a direct consequence of (b).

The rest of the subsection is devoted to the description of the complete Boolean algebras $B$ for which the notions of regular and $\sigma$-regular $B$-system coincide.

Definition 50. Given an element $c \in B$ and sets $C, D, D_{n} \subset B(n \in \mathbb{N})$, introduce the following notions:

$c$ is refined from $D$, in writing $c \preccurlyeq D$, if $c \leqslant d$ for some $d \in D$;

$C$ is refined from $D$, in writing $C \preccurlyeq D$, if $c \preccurlyeq D$ for all $c \in C$;

$c$ is strictly refined from $D$, in writing $c \prec D$, if $c<d$ for some $d \in D$;

$C$ is strictly refined from $D$, in writing $C \prec D$, if $c \prec D$ for all $c \in C$;

$c$ is refined from $\left(D_{n}\right)_{n \in \mathbb{N}}$ if $c \preccurlyeq D_{n}$ for all $n \in \mathbb{N}$;

$C$ is refined from $\left(D_{n}\right)_{n \in \mathbb{N}}$ if $C \preccurlyeq D_{n}$ for all $n \in \mathbb{N}$.

A set $C \subset B$ is called a cover of a Boolean algebra $B$ if $\vee C=1_{B}$. A partition of a Boolean algebra is a partition of unity; that is, a cover that is an antichain. Recall that, by the exhaustion principle, from each cover we can refine a partition.

Theorem 10 ([11] (Section 19), [12]). The following properties of a complete Boolean algebra B are equivalent:

(a) $\bigwedge_{n \in \omega} \bigvee_{i \in I} b(n, i)=\bigvee_{i \in I^{\omega}} \bigwedge_{n \in \omega} b(n, i(n))$ for all sets $I$ and functions $b: \omega \times I \rightarrow B$;

(b) $\bigvee_{n \in \omega} \bigwedge_{i \in I} b(n, i)=\bigwedge_{i \in I^{\omega}} \bigvee_{n \in \omega} b(n, i(n))$ for all sets I and functions $b: \omega \times I \rightarrow B$;

(c) from each sequence of covers of $B$ we can refine a cover;

(d) from each sequence of partitions of $B$ we can refine a partition;

(e) for every sequence of partitions $\left(D_{n}\right)_{n \in \mathbb{N}}$ of $B$ and every nonzero $a \in B$, there exists a sequence of $d_{n} \in D_{n}(n \in \mathbb{N})$ such that

$$
a \wedge \bigwedge_{n \in \mathbb{N}} d_{n} \neq 0_{B} .
$$

A complete Boolean algebra $B$ satisfying each of the equivalent conditions (a)-(e) is called $\omega$-distributive or $(\omega, \infty)$-distributive; see [8] (Section 14).

Every atomic complete Boolean algebra is $\omega$-distributive. The completion of the quotient Boolean algebra $\mathcal{P}(\omega) / \mathcal{P}_{\text {fin }}(\omega)$ is an atomless $\omega$-distributive complete Boolean algebra; see [12] (Corollary to Lemma 5), [13] (Example 9). A classical example of a complete Boolean algebra that is not $\omega$-distributive is given by the Boolean algebra of cosets of Lebesgue measurable subsets of $\mathbb{R}$.

Definition 51. Given a sequence $\left(C_{n}\right)_{n \in \mathbb{N}}$ of subsets of $B$, call it a refinement in $B$ if

(a) $\vee C_{n}=\vee C_{1} \neq 0_{B}$ for all $n \in \mathbb{N}$;

(b) $\Lambda_{n \in \mathbb{N}} c_{n}=0_{B}$ for every sequence of $c_{n} \in C_{n}(n \in \mathbb{N})$.

Say that a refinement $\left(C_{n}\right)_{n \in \mathbb{N}}$ is partitioning if, for all $n \in \mathbb{N}$,

(c) $0_{B} \notin C_{n}$;

(d) $C_{n}$ is an antichain;

(e) $C_{n+1} \prec C_{n}$.

The following is readily verified:

Lemma 28. Let $\left(C_{n}\right)_{n \in \mathbb{N}}$ be a partitioning refinement in $B$ and let $X:=\bigcup_{n \in \mathbb{N}} C_{n}$. 
(a) For each $x \in X$, there exists a unique finite list of elements $c_{1} \in C_{1}, c_{2} \in C_{2}, \ldots, c_{n} \in C_{n}$ such that $c_{1}>c_{2}>\cdots>c_{n}=x$.

(b) If $n \neq m$ then $C_{n} \cap C_{m}=\varnothing$.

(c) Given en element $x \in X$, denote by $h(x)$ the only $n \in \mathbb{N}$ for which $x \in C_{n}$. If $\left(x_{i}\right)_{i \in I} \subset X$ and $\bigvee_{i \in I} h\left(x_{i}\right)=\infty$, then $\bigwedge_{i \in I} x_{i}=0_{B}$.

Theorem 11. The following properties of a complete Boolean algebra B are equivalent:

(a) $B$ admits a partitioning refinement;

(b) $B$ admits a refinement;

(c) $B$ is not $\omega$-distributive.

Proof. The implication $(\mathrm{a}) \Rightarrow(\mathrm{b})$ is trivial; $(\mathrm{b}) \Rightarrow(\mathrm{c})$ is easily deduced from Theorem $10(\mathrm{e})$. We will show that $(\mathrm{c}) \Rightarrow(\mathrm{a})$.

If $B$ is not $\omega$-distributive; then, by Theorem $10(\mathrm{e})$, there exist a sequence of partitions $\left(D_{n}\right)_{n \in \mathbb{N}}$ and a nonzero $a \in B$ such that

$$
a \wedge \bigwedge_{n \in \mathbb{N}} d_{n}=0_{B}
$$

for every sequence of $d_{n} \in D_{n}(n \in \mathbb{N})$. By (24), it is impossible to refine any nonzero element $b \leqslant a$ from $\left(D_{n}\right)_{n \in \mathbb{N}}$, and so for each nonzero $b \leqslant a$ we can consider the natural

$$
m(b):=\min \left\{n \in \mathbb{N}: b \nprec D_{n}\right\} .
$$

From $\vee D_{m(b)}=1_{B}$ it follows that $b \wedge \vee D_{m(b)}=b$. Moreover, since $b \npreceq D_{m(b)}$, we have $b \wedge d<b$ for all $d \in D_{m(b)}$. Therefore, the set

$$
P(b):=\left\{b \wedge d: d \in D_{m(b)}\right\} \backslash\left\{0_{B}\right\}
$$

possesses the following properties:

$$
\begin{aligned}
& P(b) \text { is an antichain, } \\
& \vee P(b)=b, \\
& 0_{B}<c<b \text { for all } c \in P(b) .
\end{aligned}
$$

Define $C_{n} \subset B(n \in \mathbb{N})$ recursively by putting

$$
\begin{aligned}
& C_{1}:=P(a) ; \\
& C_{n+1}:=\bigcup_{b \in C_{n}} P(b), \quad n \in \mathbb{N},
\end{aligned}
$$

and demonstrate that $\left(C_{n}\right)_{n \in \mathbb{N}}$ is a partitioning refinement. Conditions (a), (c)-(e) of Definition 51 are obvious. It remains to justify Definition $51(b)$.

Show by induction on $n \in \mathbb{N}$ that $m(b) \geqslant n$ for all $b \in C_{n}$. The induction base $n=1$ is trivial. Suppose that $m(b) \geqslant n$ for all $b \in C_{n}$, consider an arbitrary $c \in C_{n+1}$, and prove that $m(c) \geqslant n+1$. By the definition of $C_{n+1}$, we have the representation $c=b \wedge d$ for some $b \in C_{n}$ and $d \in D_{m(b)}$. Since $b$ is refined from $D_{1}, \ldots, D_{m(b)-1} ;$ by the inequality $c \leqslant b$, the same holds for $c$, and so $m(c) \geqslant m(b) \geqslant n$. Moreover, from $c \npreceq D_{m(c)}$ and $c \leqslant d \in D_{m(b)}$ it follows that $m(c) \neq m(b)$, and so $m(c) \geqslant n+1$.

Now, let $c_{n} \in C_{n}$ for all $n \in \mathbb{N}$. By the above, $m\left(c_{n+1}\right)>n$, whence $c_{n+1} \preccurlyeq D_{n}$, and so $c_{n+1} \leqslant d_{n}$ for some sequence of $d_{n} \in D_{n}(n \in \mathbb{N})$. Applying (24), we conclude that

$$
\bigwedge_{n \in \mathbb{N}} c_{n}=c_{1} \wedge \bigwedge_{n \in \mathbb{N}} c_{n+1} \leqslant a \wedge \bigwedge_{n \in \mathbb{N}} d_{n}=0_{B} .
$$


Theorem 12. The notions of regular and $\sigma$-regular B-system are equivalent if and only if the Boolean algebra $B$ is $\omega$-distributive.

Proof. Necessity: If $B$ is not $\omega$-distributive; then, by Theorem 11, there exists a partitioning refinement $\left(C_{n}\right)_{n \in \mathbb{N}}$ in $B$. Put

$$
X:=\bigcup_{n \in \mathbb{N}} C_{n} .
$$

As in Lemma 28(c), given $x \in X$, denote by $h(x)$ the only $n \in \mathbb{N}$ for which $x \in C_{n}$. Turn $X$ into a $B$-system by putting for $x, y \in X$

$$
\begin{aligned}
& {[=]_{X}(x, y):= \begin{cases}1_{B} & \text { if } x=y, \\
0_{B} & \text { otherwise; }\end{cases} } \\
& {[\in]_{X}(x, y):= \begin{cases}x & \text { if } h(x)=h(y)+1, \\
0_{B} & \text { otherwise. }\end{cases} }
\end{aligned}
$$

In order to establish the $\sigma$-regularity of $X$, consider an arbitrary sequence $\left(x_{n}\right)_{n \in \mathbb{N}} \subset X$ and show that $\wedge_{n \in \mathbb{N}}\left[x_{n+1} \in x_{n}\right]=0_{B}$. This relation is obvious if $\left[x_{n+1} \in x_{n}\right]=0_{B}$ for some $n \in \mathbb{N}$. Otherwise, $h\left(x_{n+1}\right)=h\left(x_{n}\right)+1$ and $\left[x_{n+1} \in x_{n}\right]=x_{n+1}$ for all $n \in \mathbb{N}$. Then $\bigvee_{n \in \mathbb{N}} h\left(x_{n+1}\right)=\infty$, whence, by Lemma 28 (c), we have

$$
\bigwedge_{n \in \mathbb{N}}\left[x_{n+1} \in x_{n}\right]=\bigwedge_{n \in \mathbb{N}} x_{n+1}=0_{B} .
$$

The system $X$ is not regular, since

$$
P:=\left\{\left.x\right|_{x}: x \in X\right\} \subset{ }^{\%} X
$$

satisfies Lemma 25(b). Indeed, for all $n \in \mathbb{N}$ and $x \in C_{n}$,

$$
\begin{gathered}
\bigvee_{y \in X}\left[\left.\left.y\right|_{y} \in x\right|_{x}\right]=x \wedge \bigvee_{y \in X}[y \in x] \wedge y \geqslant x \wedge \bigvee_{y \in C_{n+1}}[y \in x] \wedge y \\
\quad=x \wedge \bigvee_{y \in C_{n+1}} y=x \wedge \vee C_{n+1}=x \wedge \vee C_{n}=x=\left.\Delta x\right|_{x}
\end{gathered}
$$

Sufficiency: Let $X$ be a $B$-system that is $\sigma$-regular and not regular. Consider the sets $P_{n} \subset{ }^{\%} X(n \in \mathbb{N})$ satisfying Lemma 25(c), put

$$
C_{n}:=\left\{\Delta p: p \in P_{n}\right\}, \quad n \in \mathbb{N},
$$

and show that $\left(C_{n}\right)_{n \in \mathbb{N}}$ is a refinement in $B$ (see Theorem 11). Indeed, for all $n \in \mathbb{N}$,

$$
\vee C_{n}=\bigvee_{p \in P_{n}} \Delta p=\bigvee_{p \in P_{1}} \Delta p=\vee C_{1} \neq 0_{B}
$$

Let $c_{n} \in C_{n}$ for all $n \in \mathbb{N}$. Show that

$$
\bigwedge_{n \in \mathbb{N}} c_{n}=0_{B} .
$$

The latter is obvious if $c_{n} \wedge c_{n+1}=0_{B}$ for some $n \in \mathbb{N}$. Suppose now that $c_{n} \wedge c_{n+1} \neq 0_{B}$ for all $n \in \mathbb{N}$. By (25), for each $n \in \mathbb{N}$ there exists an element $p_{n} \in P_{n}$ such that $c_{n}=\Delta p_{n}$. Since $\Delta p_{n} \wedge \Delta p_{n+1}=c_{n} \wedge c_{n+1} \neq 0_{B}$; therefore, Lemma 25(c) implies that $c_{n+1}=\Delta p_{n+1}=$ $\left[p_{n+1} \in p_{n}\right]$. Consequently,

$$
\bigwedge_{n \in \mathbb{N}} c_{n} \leqslant \bigwedge_{n \geqslant 2} c_{n}=\bigwedge_{n \in \mathbb{N}} c_{n+1}=\bigwedge_{n \in \mathbb{N}}\left[p_{n+1} \in p_{n}\right]=0_{B}
$$

owing to the $\sigma$-regularity of $X$. 


\section{The Structure of the Boolean-Valued Universe}

In this section, we introduce the notion of universe over an arbitrary extensional Boolean-valued system and establish a close interrelation between such a universe and the intensional hierarchy, a Boolean-valued analog of the von Neumann cumulative hierarchy. This general tool makes it possible to prove the uniqueness of the classical Boolean-valued universe $\mathbb{V}^{(B)}$ up to a unique isomorphism and to construct examples of Boolean-valued systems with unusual properties. In particular, given an arbitrary complete Boolean algebra $B$, we show that the conditions listed in the axiomatic characterization of $\mathbb{V}^{(B)}$ are logically independent. We also describe the structure of $\mathbb{V}^{(B)}$ by means of four cumulative hierarchies.

\subsection{Intensional Hierarchy}

The von Neumann cumulative hierarchy $\left(V_{\alpha}\right)_{\alpha \in \text { Ord }}$ over a set or a class $V_{0}$ is defined by the transitive recursion

$$
\begin{array}{ll}
V_{\alpha+1}=V_{\alpha} \cup \mathcal{P}\left(V_{\alpha}\right), & \alpha \in \text { Ord } \\
V_{\alpha}=\bigcup_{\beta<\alpha} V_{\beta}, & \alpha \in \text { Lim Ord. }
\end{array}
$$

In this subsection, we define an intensional hierarchy that serves as an analog of the hierarchy (26) for Boolean-valued systems, introduce the notion of Boolean-valued universe over an arbitrary extensional Boolean-valued system, and establish a close relationship of such a universe with the corresponding intensional hierarchy.

Definition 52. We begin with a characterization of the superstructure that is a Boolean-valued analog of the discrete step $V_{\alpha+1}=V_{\alpha} \cup \mathcal{P}\left(V_{\alpha}\right)$ of (26).

Given an extensional B-system $X$ and an arbitrary subclass $Y \subset X$, introduce the following notions:

$$
\begin{aligned}
X \text { is intensional over } Y & \Leftrightarrow\left(\forall P \subset{ }^{\%} Y\right)(\exists x \in X)(x \simeq P \uparrow) \\
& \Leftrightarrow(\forall \Phi \Subset X: \Phi \leqslant Y \uparrow)(\exists x \in X)(x \simeq \Phi) ; \\
X \text { is predicative over } Y & \Leftrightarrow(\forall x \in X \backslash Y)\left(\exists P \subset{ }^{\%} Y\right)(x \simeq P \uparrow) \\
& \Leftrightarrow X=Y \cup \mathcal{P}_{X}(Y) ; \\
X \text { is separated over } Y & \Leftrightarrow(\forall x \in X)(\forall z \in X \backslash Y)(x \simeq z \Rightarrow x=z) \\
& \Leftrightarrow\left(\forall x_{1}, x_{2} \in X\right)\left(x_{1} \simeq x_{2}, x_{1} \neq x_{2} \Rightarrow x_{1}, x_{2} \in Y\right) .
\end{aligned}
$$

Say that a B-system $X$ is a superstructure over a subsystem $Y$ if
(a) $Y \preccurlyeq X$;
(b) $X$ is extensional;
(c) $X$ is intensional over $Y$;
(d) $X$ is predicative over $Y$;
(e) $X$ is separated over $Y$.

Refer to $X$ as a superstructure over a copy of a B-system $Z$ if $X$ is a superstructure over a subsystem isomorphic to $\mathrm{Z}$.

Lemma 29. For every extensional Boolean-valued system $Z$, there exists a superstructure over a copy of $Z$.

Proof. Consider an arbitrary extensional $B$-system $Z$, its isomorphic copy $Y:=\{\varnothing\} \times Z$, and put (see Definition 32)

$$
\mathcal{Y}:=\left\{P \subset{ }^{\%} Y: P \text { is saturated, } \neg(\exists y \in Y)\left(y \simeq P \uparrow_{Y}\right)\right\} .
$$


Note that $Y \cap \mathcal{Y}=\varnothing$. Indeed, by Definition 23, the elements of every subset $P \subset{ }^{\%} Y$ are sets of pairs, whereas every element of the product $Y=\{\varnothing\} \times Z$ in Kuratowski's approach has the form $\{\{\varnothing\},\{\varnothing, z\}\}$ and so contains $\{\varnothing\}$ that is not a set of pairs.

Put $X:=Y \cup \mathcal{Y}$ and extend the interpretations $[=]_{Y},[\in]_{Y}$ onto $X^{2}$ by putting

$$
\begin{aligned}
{[=]_{X}(y, z) } & :=[=]_{Y}(y, z), \\
{[\in]_{X}(y, z) } & :=[\in]_{Y}(y, z) ; \\
{[=]_{X}(P, Q) } & :=\left[P \uparrow_{Y}=Q \uparrow_{Y}\right]_{Y}, \\
{[\in]_{X}(P, Q) } & :=\left[P \uparrow_{Y} \in Q \uparrow_{Y}\right]_{Y} ; \\
{[=]_{X}(P, y) } & :=\left[P \uparrow_{Y}=y\right]_{Y} \\
{[\in]_{X}(P, y) } & :=\left[P \uparrow_{Y} \in y\right]_{Y} ; \\
{[=]_{X}(y, P) } & :=\left[y=P \uparrow_{Y}\right]_{Y} \\
{[\in]_{X}(y, P) } & :=\left[y \in P \uparrow_{Y}\right]_{Y}
\end{aligned}
$$

for all $y, z \in Y$ and $P, Q \in \mathcal{Y}$.

The fact that $X$ is a $B$-system under the interpretations $[=]_{X}$ and $[\in]_{X}$ is established by an elementary check of the conditions of Definition 26. In most cases the syntactic sugar of Definition 22 and the validity in $Y$ of the propositional and equality axioms (see Proposition 11) are enough for this check. We will only clarify five cases, in three of which the extensionality of $Y$ is used, and the other two employ the obvious inequality $[\varphi(x)]_{Y} \leqslant[(\exists x) \varphi(x)]_{Y}$.

If $x, y, z \in Y$ and $P, Q \in \mathcal{Y}$ then

$$
\begin{aligned}
& {[=]_{X}(x, P) \wedge[=]_{X}(P, z)=\left[x=P \uparrow_{Y} \wedge P \uparrow_{Y}=z\right]_{Y}} \\
& \quad=\left[(\forall y)\left(y \in x \Leftrightarrow y \in P \uparrow_{Y}\right) \wedge(\forall y)\left(y \in P \uparrow_{Y} \Leftrightarrow y \in z\right)\right]_{Y} \\
& \quad \leqslant[(\forall y)(y \in x \Leftrightarrow y \in z)]_{Y} \leqslant[x=z]_{Y}=[=]_{X}(x, z) ; \\
& {[\in]_{X}(P, y) \wedge[=]_{X}(P, z)=\left[P \uparrow_{Y} \in y \wedge P \uparrow_{Y}=z\right]_{Y}} \\
& \quad=\left[(\exists x)\left(x=P \uparrow_{Y} \wedge x \in y\right) \wedge P \uparrow_{Y}=z\right]_{Y} \\
& \quad=\left[(\exists x)\left((\forall u)\left(u \in x \Leftrightarrow u \in P \uparrow_{Y}\right) \wedge x \in y\right) \wedge(\forall u)\left(u \in P \uparrow_{Y} \Leftrightarrow u \in z\right)\right]_{Y} \\
& \quad \leqslant[(\exists x)((\forall u)(u \in x \Leftrightarrow u \in z) \wedge x \in y)]_{Y} \\
& \quad \leqslant[(\exists x)(x=z \wedge x \in y)]_{Y} \leqslant[z \in y]_{Y}=[\in]_{X}(z, y) ; \\
& \quad[\in]_{X}(P, Q) \wedge[=]_{X}(P, z)=\left[P \uparrow_{Y} \in Q \uparrow_{Y} \wedge P \uparrow_{Y}=z\right]_{Y} \\
& \quad=\left[(\exists x)\left(x=P \uparrow_{Y} \wedge x \in Q \uparrow_{Y}\right) \wedge P \uparrow_{Y}=z\right]_{Y} \\
& \quad=\left[(\exists x)\left((\forall y)\left(y \in x \Leftrightarrow y \in P \uparrow_{Y}\right) \wedge x \in Q \uparrow_{Y}\right) \wedge(\forall y)\left(y \in P \uparrow_{Y} \Leftrightarrow y \in z\right)\right]_{Y} \\
& \quad \leqslant\left[(\exists x)\left((\forall y)(y \in x \Leftrightarrow y \in z) \wedge x \in Q \uparrow_{Y}\right)\right]_{Y} \\
& \quad \leqslant\left[(\exists x)\left(x=z \wedge x \in Q \uparrow_{Y}\right)\right]_{Y} \leqslant\left[z \in Q \uparrow_{Y}\right]_{Y}=[\in]_{X}(z, Q) ; \\
& {[\in]_{X}(x, y) \wedge[=]_{X}(x, P)=\left[x \in y \wedge x=P \uparrow_{Y}\right]_{Y}} \\
& \quad \leqslant\left[(\exists x)\left(x=P \uparrow_{Y} \wedge x \in y\right)\right]_{Y}=\left[P \uparrow_{Y} \in y\right]_{Y}=[\in]_{X}(P, y) ; \\
& {[\in]_{X}(x, P) \wedge[=]_{X}(x, Q)=\left[x \in P \uparrow_{Y} \wedge x=Q \uparrow_{Y}\right]_{Y}} \\
& \quad \leqslant\left[(\exists x)\left(x=Q \uparrow_{Y} \wedge x \in P \uparrow_{Y}\right)\right]_{Y}=\left[Q \uparrow_{Y} \in P \uparrow_{Y}\right]_{Y}=[\in]_{X}(Q, P) .
\end{aligned}
$$


Definition 52(a): Establish the transitivity of the subsystem $Y \subset X$ by checking condition (c) of Proposition 17. Suppose that $u \in X$ and $v \in Y$. If $u \in Y$ then $[u \in v]_{X} \leqslant$ $1_{B}=\left[u \in Y \uparrow_{X}\right]_{X}$, and if $u \in \mathcal{Y}$ then

$$
\begin{gathered}
{[u \in v]_{X}=\left[u \uparrow_{Y} \in v\right]_{Y}=\left[(\exists y)\left(y=u \uparrow_{Y} \wedge y \in v\right)\right]_{Y}} \\
\leqslant\left[(\exists y)\left(u \uparrow_{Y}=y\right)\right]_{Y}=\bigvee_{y \in Y}\left[u \uparrow_{Y}=y\right]_{Y}=\bigvee_{y \in Y}[u=y]_{X}=\left[u \in Y \uparrow_{X}\right]_{X} .
\end{gathered}
$$

Definition 52(b): If $u, v \in X$ and $[\cdot \in u]_{X}=[\cdot \in v]_{X}$; then, in each of the three cases

$$
u, v \in Y ; \quad u \in Y, v \in \mathcal{Y} ; \quad u, v \in \mathcal{Y},
$$

we have

$$
[u=v]_{X}=\left\{\begin{array}{ll}
{[u=v]_{Y}} & =\bigwedge_{z \in Y}[z \in u]_{Y} \Leftrightarrow_{B}[z \in v]_{Y} \\
{\left[u=v \uparrow_{Y}\right]_{Y}} & =\bigwedge_{z \in Y}[z \in u]_{Y} \Leftrightarrow_{B}\left[z \in v \uparrow_{Y}\right]_{Y} \\
{\left[u \uparrow_{Y}=v \uparrow_{Y}\right]_{Y}=\bigwedge_{z \in Y}\left[z \in u \uparrow_{Y}\right]_{Y} \Leftrightarrow_{B}\left[z \in v \uparrow_{Y}\right]_{Y}}
\end{array}\right\}=\bigwedge_{z \in Y}[z \in u]_{X} \Leftrightarrow \Leftrightarrow_{B}[z \in v]_{X}=1_{B} .
$$

The following relation in $X$ will be of use below:

$$
P \simeq P \uparrow_{X} \text { for all } P \in \mathcal{Y} .
$$

Let $P=\left\{\left.y_{i}\right|_{b_{i}}: i \in I\right\} \in \mathcal{Y}$. Demonstrate that $[x \in P]_{X}=\left[x \in P \uparrow_{X}\right]_{X}$ for all $x \in X$. Indeed, if $y \in Y$ then $[y \in P]_{X}=\left[y \in P \uparrow_{Y}\right]_{Y}=\left[y \in P \uparrow_{X}\right]_{X}$ (see Remark 16 or Theorem 8); and if $Q \in \mathcal{Y}$ then by Lemma 4

$$
\begin{gathered}
{[Q \in P]_{X}=\left[Q \uparrow_{Y} \in P \uparrow_{Y}\right]_{Y}=\bigvee_{p \in P}\left[Q \uparrow_{Y}=p\right]_{Y}} \\
=\bigvee_{i \in I}\left[Q \uparrow_{Y}=y_{i}\right]_{Y} \wedge b_{i}=\bigvee_{i \in I}\left[Q=y_{i}\right]_{X} \wedge b_{i}=\left[Q \in P \uparrow_{X}\right]_{X} .
\end{gathered}
$$

Definition 52(c): Consider a subset $P \subset{ }^{\%} Y$ and show that $x \simeq P \uparrow_{X}$ for some $x \in X$. Let $\bar{P}:=P \uparrow_{Y} \Downarrow_{Y} \subset{ }^{\%} Y$ be the saturated hull of $P$ in $Y$ (see Corollary 3). Since $P \subset \bar{P} \subset$ $P \uparrow \Downarrow_{X}$; therefore, $P \uparrow_{X} \leqslant \bar{P} \uparrow_{X} \leqslant P \uparrow_{X} \Downarrow_{X} \uparrow_{X}$, and so $P \uparrow_{X}=\bar{P} \uparrow_{X}$ by the equality $P \uparrow_{X} \Downarrow_{X} \uparrow_{X}=P \uparrow_{X}$ (see Lemma 7). If $y \simeq \bar{P} \uparrow_{Y}$ in $Y$ for some $y \in Y$; then $y \simeq \bar{P} \uparrow_{X}$ in $X$ by Proposition 18, and so $x:=y \simeq \bar{P} \uparrow_{X}=P \uparrow_{X}$. If $\neg(\exists y \in Y)\left(y \simeq \bar{P} \uparrow_{Y}\right)$ then $\bar{P} \in \mathcal{Y}$ and, in this case, $x:=\bar{P} \simeq \bar{P} \uparrow_{X}=P \uparrow_{X}$ owing to (27).

Definition 52 (d): This is a direct consequence of (27).

Definition 52 (e): Suppose that $y \in Y$ and $P \in \mathcal{Y}$. By the definition of $\mathcal{Y}$, we have $y \not P \uparrow_{Y}$ in $Y$; whence from Proposition 18 it follows that $y \not f P \uparrow_{X}$ in $X$ and so $y \not P$ by (27).

Now, let $P, Q \in \mathcal{Y}, P \simeq Q$. The relation (27) implies $P \uparrow_{X}=Q \uparrow_{X}$. Then $P \uparrow_{Y}=\left.\left(P \uparrow_{X}\right)\right|_{Y}=$ $\left.\left(Q \uparrow_{X}\right)\right|_{Y}=Q \uparrow_{Y}$. Since $P$ and $Q$ are saturated subsets of $\%$; according to Theorem 3 we have $P=P \uparrow_{Y} \Downarrow_{Y}=Q \uparrow_{Y} \Downarrow_{Y}=Q$.

\section{Lemma 30.}

(a) If a B-system $X$ is a superstructure over a subsystem $Y \subset X, f: X \leftrightarrow_{B} X$, and $\left.f\right|_{Y}=\operatorname{id}_{Y}$; then $f=\operatorname{id}_{X}$.

(b) If B-systems $X$ and $X^{\prime}$ are superstructures over subsystems $Y \subset X$ and $Y^{\prime} \subset X^{\prime}$ then each isomorphism $f: Y \leftrightarrow_{B} Y^{\prime}$ extends to a unique isomorphism $\bar{f}: X \leftrightarrow_{B} X^{\prime}$. In particular, a superstructure over a copy of a Boolean-valued system is unique up to isomorphism. 
Proof. (a): Observe first of all that conditions (b)-(d) of Definition 52 imply the relation

$$
\left(\forall x_{1}, x_{2} \in X \backslash Y\right)\left[x_{1}=x_{2}\right]_{X}=\bigwedge_{y \in Y}\left[y \in x_{1} \Leftrightarrow y \in x_{2}\right]_{X} .
$$

Indeed, for every $x \in X \backslash Y$, there is a subset $P \subset{ }^{\%} Y$ such that $X \vDash\left(x=P \uparrow_{X}\right)$, which in view of the validity $X \vDash\left(P \uparrow_{X} \subset Y \uparrow_{X}\right)$ (see Lemma $\left.5(b)\right)$ implies $X \vDash\left(x \subset Y \uparrow_{X}\right)$. Consequently,

$$
X \vDash\left(x_{1}=x_{2} \Leftrightarrow\left(\forall y \in Y \uparrow_{X}\right)\left(y \in x_{1} \Leftrightarrow y \in x_{2}\right)\right)
$$

for all $x_{1}, x_{2} \in X \backslash Y$; and it remains to refer to Corollary 2.

Suppose that $X, Y$, and $f$ satisfy the conditions of (a). Consider an arbitrary $x \in X \backslash Y$ and show that $f(x)=x$. It is clear that $f(x) \in X \backslash Y$. Reckoning with (28) and the equality $f(y)=y$ for $y \in Y$, we conclude that

$$
\begin{gathered}
{[f(x)=x]_{X}=\bigwedge_{y \in Y}[y \in f(x) \Leftrightarrow y \in x]_{X}} \\
=\bigwedge_{y \in Y}[f(y) \in f(x)]_{X} \Leftrightarrow_{B}[y \in x]_{X}=\bigwedge_{y \in Y}[y \in x]_{X} \Leftrightarrow_{B}[y \in x]_{X}=1_{B} .
\end{gathered}
$$

Thus, $f(x) \simeq x$, which by Definition $52(\mathrm{e})$ implies $f(x)=x$.

(b): The uniqueness of an extension $\bar{f}$ follows from (a). Prove the existence. Consider arbitrary extensional $B$-systems $X$ and $X^{\prime}$, that are superstructures over subsystems $Y \subset X$ and $Y^{\prime} \subset X^{\prime}$, and an isomorphism $f: Y \leftrightarrow_{B} Y^{\prime}$.

Consider an arbitrary element $x \in X \backslash Y$. By Definition $52(\mathrm{~d})$, there is a set $P \subset{ }^{\%} Y$ such that $x \simeq P \uparrow_{X}$. Due to the relations $P \subset P \uparrow_{X} \Downarrow_{X}$ and $P \uparrow_{X}=P \uparrow_{X} \Downarrow_{X} \uparrow_{X}$ (see Lemma 7), we have

$$
P \uparrow_{X}=\left(P \uparrow_{X} \Downarrow_{X} \cap P\right) \uparrow_{X} \leqslant\left(P \uparrow_{X} \Downarrow_{X} \cap \%\right) \uparrow_{X} \leqslant P \uparrow_{X} \Downarrow_{X} \uparrow_{X}=P \uparrow_{X}
$$

and so $x \simeq P \uparrow_{X}=\left(P \uparrow_{X} \Downarrow_{X} \cap{ }^{\%} Y\right) \uparrow_{X}=\left(x \Downarrow_{X} \cap{ }^{\%} Y\right) \uparrow_{X}$. Moreover, since $P$ is a set, Theorem 4 implies that the class $x \Downarrow_{X}=P \uparrow_{X} \Downarrow_{X}$ is a set too. Therefore, by putting

$$
P_{x}:=x \Downarrow_{X} \cap{ }^{\%} Y, \quad x \in X \backslash Y,
$$

we obtain a definable class family of subsets $P_{x} \subset{ }^{\%} Y$ such that $x \simeq P_{x} \uparrow_{X}$ for all $x \in X \backslash Y$.

Next, given $x \in X \backslash Y$, put $P_{x}^{\prime}:=f^{\%}\left(P_{x}\right) \subset{ }^{\%} Y^{\prime}$ (see Definition 30). By Definition 52 (c), there exists an element $z \in X^{\prime}$ such that $z \simeq P_{x}^{\prime} \uparrow_{X^{\prime}}$. Note that $z$ cannot belong to $Y^{\prime}$. Indeed, if $z \in Y^{\prime}$ then $y:=f^{-1}(z) \in Y$ and, using Propositions 13 and 18 and conditions (a), (b) and (e) of Definition 52, we infer the following chain of implications leading to a contradiction:

$$
\begin{aligned}
z \simeq P_{x}^{\prime} \uparrow_{X^{\prime}} & \Rightarrow f(y) \simeq f^{\%}\left(P_{x}\right) \uparrow_{Y^{\prime}} \Rightarrow Y^{\prime} \vDash\left(f(y)=f^{\%}\left(P_{x}\right) \uparrow_{Y^{\prime}}\right) \Rightarrow Y \vDash\left(y=P_{x} \uparrow_{Y}\right) \\
& \Rightarrow X \vDash\left(y=P_{x} \uparrow_{X}\right) \Rightarrow y \simeq P_{x} \uparrow_{X} \Rightarrow x \simeq y \Rightarrow x=y \Rightarrow x \in Y .
\end{aligned}
$$

Taking account of Definition 52 (e), we conclude that, for each $x \in X \backslash Y$, there is a unique element $z \in X^{\prime} \backslash Y^{\prime}$ such that $z \simeq P_{x}^{\prime} \uparrow_{X^{\prime}}$. Therefore, we obtain a definable class function $g: X \backslash Y \rightarrow X^{\prime} \backslash Y^{\prime}$ satisfying $g(x) \simeq P_{x}^{\prime \prime} \uparrow_{X^{\prime}}$ for all $x \in X \backslash Y$.

Define $\bar{f}: X \rightarrow X^{\prime}$ by putting

$$
\bar{f}(x):= \begin{cases}f(x) & \text { if } x \in Y, \\ g(x) & \text { if } x \in X \backslash Y,\end{cases}
$$

and show that $\bar{f}: X \leftrightarrow_{B} X^{\prime}$. For convenience, put $x^{\prime}:=\bar{f}(x) \in X^{\prime}$ for each $x \in X$. Therefore, $f: y \mapsto y^{\prime}$ is an isomorphism from $Y$ onto $Y^{\prime}$, and, for all $x \in X \backslash Y$,

$$
\begin{aligned}
& P_{x} \subset{ }^{\%} Y, \quad P_{x}^{\prime}=f^{\%}\left(P_{x}\right) \subset{ }^{\%} Y^{\prime}, \\
& x \simeq P_{x} \uparrow_{X}, \quad x^{\prime} \simeq P_{x}^{\prime} \uparrow_{X^{\prime}} .
\end{aligned}
$$


Show that $\bar{f}$ preserves the truth values of atomic formulas. Let $\varphi\left(x_{1}, x_{2}, y_{1}, y_{2}\right)$ be any of the (bounded) formulas $x_{1}=x_{2}, x_{1} \in x_{2}, x_{1}=y_{1}, x_{1} \in y_{1}, y_{1} \in x_{1}, y_{1}=y_{2}$, or $y_{1} \in y_{2}$. Then, by Proposition 13 and Theorem 8 , for all $x_{1}, x_{2} \in X \backslash Y$ and $y_{1}, y_{2} \in Y$, we have

$$
\begin{aligned}
& { }_{x}=\left[\varphi\left(P_{x_{1}} \uparrow_{X^{\prime}}, P_{x_{2}} \uparrow_{X}, y_{1}, y_{2}\right)\right]_{X}=\left[\varphi\left(P_{x_{1}} \uparrow_{Y}, P_{x_{2}} \uparrow_{Y}, y_{1}, y_{2}\right)\right]_{Y} \\
& =\left[\varphi\left(P_{x_{1}}^{\prime} \uparrow_{\gamma^{\prime}}, P_{x_{2}}^{\prime} \uparrow_{\gamma^{\prime}}, y_{1}^{\prime}, y_{2}^{\prime}\right)\right]_{Y^{\prime}}=\left[\varphi\left(P_{x_{1}}^{\prime} \uparrow_{X^{\prime}}, P_{x_{2}}^{\prime} \uparrow_{X^{\prime}}, y_{1}^{\prime}, y_{2}^{\prime}\right)\right]_{X^{\prime}}=\left[\varphi\left(x_{1}^{\prime}, x_{2}^{\prime}, y_{1}^{\prime}, y_{2}^{\prime}\right)\right]_{X^{\prime}} .
\end{aligned}
$$

Show that $\bar{f}: X \rightarrow X^{\prime}$ is injective. The injectivity of $\bar{f}$ on $Y$ is guaranteed by the injectivity of the isomorphism $f: Y \rightarrow Y^{\prime}$. If at least one of the elements $x_{1}, x_{2} \in X$ does not belong to $Y$; then since $\left[x_{1}=x_{2}\right]_{X}=\left[x_{1}^{\prime}=x_{2}^{\prime}\right]_{X^{\prime}}$ (see (29)) from $x_{1}^{\prime}=x_{2}^{\prime}$ it follows that $x_{1} \simeq x_{2}$, and so $x_{1}=x_{2}$ by Definition $52(\mathrm{e})$.

Finally, prove the surjectivity of $\bar{f}: X \rightarrow X^{\prime}$. Consider an arbitrary $z \in X^{\prime}$ and show that $z=x^{\prime}$ for some $x \in X$. If $z \in Y^{\prime}$ then the desired $x \in X$ exists by the surjectivity of the isomorphism $f: Y \rightarrow Y^{\prime}$. Let $z \in X^{\prime} \backslash Y^{\prime}$. By Definition $52(\mathrm{~d})$, there is a subset $P^{\prime} \subset^{\%} Y^{\prime}$ for which $z \simeq P^{\prime} \uparrow_{X^{\prime}}$. Put $P:=\left(f^{\%}\right)^{-1}\left(P^{\prime}\right) \subset Y$. By Definition 52 (c), there exists $x \in X$ satisfying $x \simeq P \uparrow_{X}$. If $x \in Y$; then from Propositions 13 and 18 we infer

$$
x \simeq P \uparrow_{X} \Rightarrow x \simeq P \uparrow_{Y} \Rightarrow x^{\prime} \simeq P^{\prime} \uparrow_{Y^{\prime}} \Rightarrow x^{\prime} \simeq P^{\prime} \uparrow_{X^{\prime}} \Rightarrow x^{\prime} \simeq z,
$$

which contradicts Definition 52 (e). Consequently, $x \notin Y$. So, by Propositions 13 and 18,

$$
x \simeq P \uparrow_{X} \Rightarrow P_{x} \uparrow_{X}=P \uparrow_{X} \Rightarrow P_{x} \uparrow_{Y}=P \uparrow_{Y} \Rightarrow P_{x}^{\prime} \uparrow_{Y^{\prime}}=P^{\prime} \uparrow_{Y^{\prime}} \Rightarrow P_{x}^{\prime} \uparrow_{X^{\prime}}=P^{\prime} \uparrow_{X^{\prime}} \Rightarrow x^{\prime} \simeq z .
$$

Owing to Definition $52(\mathrm{e})$, the latter implies $x^{\prime}=z$.

Definition 53. Say that a family of extensional B-systems $\left(X_{\alpha}\right)_{\alpha \in \mathrm{O} d \mathrm{O}^{\bullet}}$ is an intensional hierarchy or, more exactly, a B-valued intensional hierarchy over $X_{0}$ if

$$
\begin{array}{ll}
X_{\alpha+1} \text { is a superstructure over } X_{\alpha}, & \alpha \in \text { Ord; } \\
X_{\alpha}=\bigcup_{\beta<\alpha} X_{\beta}, & \alpha \in \text { Lim Ord } .
\end{array}
$$

In this case, $X_{\beta} \preccurlyeq X_{\alpha}$ for all $\beta \leqslant \alpha \in$ Ord $^{\bullet}$ (see Proposition $19(\mathrm{e})$ ).

Lemma 31. For every extensional B-system $Y$, there exists an intensional hierarchy $\left(X_{\alpha}\right)_{\alpha \in \text { Ord }}$ • such that $X_{0} \leftrightarrow_{B} Y$.

Proof. The desired hierarchy can be easily built on using Proposition 19 and Lemma 29 together with the construction of the direct limit; see, for example, [14] (III.7.5). Indeed, define the family of $B$-systems $Y_{\alpha}\left(\alpha \in\right.$ Ord $\left.^{*}\right)$ and isomorphic embeddings $f_{\beta}^{\alpha}: Y_{\beta} \rightarrow Y_{\alpha}$ $\left(\beta \leqslant \alpha \in\right.$ Ord $\left.^{*}\right)$ satisfying the relations $f_{\alpha}^{\alpha}=\operatorname{id}_{Y_{\alpha}}$ and $f_{\gamma}^{\alpha}=f_{\beta}^{\alpha} \circ f_{\gamma}^{\beta}\left(\gamma \leqslant \beta \leqslant \alpha \in \mathrm{Ord}^{*}\right)$, by means of the following recursive procedure:

$$
Y_{0}:=Y, f_{0}^{0}:=\operatorname{id}_{Y_{0}} ;
$$

for $\alpha \in$ Ord

$Y_{\alpha+1}$ is a superstructure over a copy of $Y_{\alpha}$ (see Definition 52 and Lemma 29),

$f_{\alpha}^{\alpha+1}$ is the corresponding isomorphism of $Y_{\alpha}$ onto a subsystem of $Y_{\alpha+1}$,

$f_{\alpha+1}^{\alpha+1}:=\operatorname{id}_{Y_{\alpha+1}}, f_{\beta}^{\alpha+1}:=f_{\alpha}^{\alpha+1} \circ f_{\beta}^{\alpha}$ for $\beta<\alpha+1$;

for $\alpha \in \operatorname{Lim}$ Ord ${ }^{\bullet}$

$$
\begin{aligned}
& Y_{\alpha}:=\left(\bigcup_{\beta<\alpha}\{\beta\} \times Y_{\beta}\right) / \sim, \text { where, for } \beta, \gamma<\alpha, \mu:=\max \{\beta, \gamma\}, x \in Y_{\beta}, y \in Y_{\gamma}, \\
&(\beta, x) \sim(\gamma, y) \Leftrightarrow f_{\beta}^{\mu}(x)=f_{\gamma}^{\mu}(y), \\
& {[=]_{\gamma_{\alpha}}(\sim(\beta, x), \sim(\gamma, y)):=[=]_{\gamma_{\mu}}\left(f_{\beta}^{\mu}(x), f_{\gamma}^{\mu}(y)\right), } \\
& {[\in]_{\gamma_{\alpha}}(\sim(\beta, x), \sim(\gamma, y)):=[\in]_{\gamma_{\mu}}\left(f_{\beta}^{\mu}(x), f_{\gamma}^{\mu}(y)\right), } \\
& f_{\alpha}^{\alpha}:=\operatorname{id}_{\gamma_{\alpha}} f_{\beta}^{\alpha}(x):=\sim(\beta, x) \text { for } \beta<\alpha, x \in Y_{\beta} .
\end{aligned}
$$

From Propositions 18 and 19 and Definition 52 it follows that the family of $B$-systems $X_{\alpha}:=f_{\alpha}^{\infty}\left(Y_{\alpha}\right)\left(\alpha \in \mathrm{Ord}^{\bullet}\right)$ is the desired intensional hierarchy. 
Lemma 32. Let $\left(X_{\alpha}\right)_{\alpha \in \mathrm{Ord}} \bullet$ and $\left(Y_{\alpha}\right)_{\alpha \in \mathrm{Ord}} \bullet$ be B-valued intensional hierarchies.

(a) If $f: X_{\infty} \leftrightarrow_{B} Y_{\infty}$ and $\left.f\right|_{X_{0}}: X_{0} \leftrightarrow_{B} Y_{0}$ then $\left.f\right|_{X_{\alpha}}: X_{\alpha} \leftrightarrow_{B} Y_{\alpha}$ for all $\alpha \in$ Ord".

(b) If $f, g: X_{\infty} \leftrightarrow_{B} Y_{\infty}$ and $\left.f\right|_{X_{0}}=\left.g\right|_{X_{0}}$ then $\left.f\right|_{X_{\alpha}}=\left.g\right|_{X_{\alpha}}$ for all $\alpha \in$ Ord $^{*}$.

(c) Every isomorphism $f_{0}: X_{0} \leftrightarrow_{B} Y_{0}$ extends to a unique isomorphism $f: X_{\infty} \leftrightarrow_{B} Y_{\infty}$.

Proof. (a) and (b): In employing induction on $\alpha \in$ Ord $^{\bullet}$, the base and limit steps are trivial, and the step $\alpha \mapsto \alpha+1$ is easy to justify for item (a) with the use of Proposition 13 and conditions (c) and (d) of Definition 52, and for item (b), with the use of Lemmas 32 (a) and 30 (a).

(c): Employing transfinite recursion and basing on Lemma 30 (b), it is easy to construct a family of isomorphisms $f_{\alpha}: X_{\alpha} \leftrightarrow_{B} Y_{\alpha}\left(\alpha \in\right.$ Ord $\left.^{*}\right)$ such that $f_{\alpha} \subset f_{\beta}$ for $\alpha \leqslant \beta$. Then $f:=f_{\infty}$ is a desired isomorphism. The uniqueness of the extension follows from (b).

Definition 54. Say that a B-system $X$ is a Boolean-valued (B-valued) universe over $X_{0}$ if the following conditions are fulfilled:

(a) $X_{0} \preccurlyeq X$;

(b) $X$ is extensional;

(c) $X$ is intensional;

(d) the elements of $X \backslash X_{0}$ are predicative;

(e) $X$ is separated over $X_{0}$;

(f) $X$ is regular outside $X_{0}$.

By Corollary 9, conditions (b) and (c) imply the cyclicity of the class $\mathcal{P}_{X}(X)$ of all predicative elements in $X$, which in view of (d) implies that the difference $X \backslash X_{0}$ is precyclic, and hence, by Theorem $9(\mathrm{~b})$, condition (f) is equivalent to the $\sigma$-regularity of $X$ outside $X_{0}$.

As is easy to see, every Boolean-valued universe $X$ over $X_{0}$ is a Boolean-valued universe over an arbitrary subsystem $Y$ satisfying the relations $X_{0} \subset Y \preccurlyeq X$.

\section{Theorem 13.}

(a) If $\left(X_{\alpha}\right)_{\alpha \in \mathrm{Ord}}$ is an intensional hierarchy then $X_{\infty}$ is a Boolean-valued universe over $X_{0}$.

(b) If $X$ is a Boolean-valued universe over $X_{0}$ then there exists a unique intensional hierarchy $\left(X_{\alpha}\right)_{\alpha \in \text { Ord }} \bullet$ such that $X=X_{\infty}$. Moreover,

$$
\begin{array}{ll}
X_{\alpha+1}=X_{\alpha} \cup \mathcal{P}_{X}\left(X_{\alpha}\right), & \alpha \in \text { Ord; } \\
X_{\alpha}=\bigcup_{\alpha<\beta} X_{\beta}, & \alpha \in \text { Lim Ord }^{\bullet} .
\end{array}
$$

Proof. (a): Verify conditions (a)-(f) of Definition 54 for $X=X_{\infty}$.

Definition 54 (a): By Proposition 19 (e), the system $X_{0}$, as well as each of the systems $X_{\alpha}$, is a transitive subsystem of $X_{\infty}$. Note that, for this reason, the fulfillment of the relation $x \simeq P \uparrow$ in one of the systems $X_{\alpha}$ is equivalent to its fulfillment in $X_{\infty}$ (see Proposition 18).

Definition 54(b) is included in the definition of intensional hierarchy.

Definition 54(c): Let $P$ be a subset of ${ }^{\%} X_{\infty}=\bigcup_{\alpha \in \text { Ord }}{ }^{\%} X_{\alpha}$. Choosing for each element $p \in P$ an ordinal $\alpha(p)$ satisfying the condition $p \in{ }^{\%} X_{\alpha(p)}$, we conclude that $P \subset{ }^{\%} X_{\alpha}$, where $\alpha:=\vee\{\alpha(p): p \in P\}$, and so $P \uparrow \simeq x$ for some $x \in X_{\alpha+1}$ by Definition 52 (c).

Definition 54(d): If $x \in X_{\infty} \backslash X_{0}$ then $x \in X_{\alpha+1}$ for some $\alpha \in$ Ord, and, consequently, $\left(\exists P \subset{ }^{\%} X_{\alpha}\right)(x \simeq P \uparrow)$ by Definition $52(\mathrm{~d})$.

Definition 54(e): Suppose that $x, y \in X_{\infty}, x \simeq y$, and $x \neq y$. Put

$$
\alpha:=\min \left\{\beta \in \text { Ord }: x, y \in X_{\beta}\right\}
$$

and show that $\alpha=0$. Indeed, $\alpha$ cannot be a limit ordinal; since, in this case $X_{\alpha}=\bigcup_{\beta<\alpha} X_{\beta}$, and so there is an ordinal $\beta<\alpha$ satisfying $x, y \in X_{\beta}$. If $\alpha=\beta+1$ then $x, y \in X_{\beta}$ by Definition $52(\mathrm{e})$. 
Definition 54(f): Prove that all the systems $X_{\alpha}$ (including $X_{\infty}$ ) are regular outside $X_{0}$ by inducting on $\alpha \in$ Ord $^{\bullet}$.

The case of $\alpha=0$ is trivial: $X_{0}$ is obviously regular outside $X_{0}$.

Suppose that $X_{\alpha}$ is regular outside $X_{0}$. By Lemma 27, for proving the regularity of $X_{\alpha+1}$ outside $X_{0}$, it suffices to demonstrate the regularity of $X_{\alpha+1}$ outside $X_{\alpha}$. By Lemma 5(b); Definition 52 (d) implies that

$$
X_{\alpha+1} \vDash(\forall y)\left(y \in X_{\alpha} \uparrow \vee y \subset X_{\alpha} \uparrow\right) .
$$

Consider an arbitrary Boolean-valued class $\Phi \Subset X_{\alpha+1}$ and prove the validity

$$
X_{\alpha+1} \vDash\left(\Phi \cap X_{\alpha} \uparrow=\varnothing \wedge \Phi \neq \varnothing \Rightarrow(\exists y \in \Phi)(y \cap \Phi=\varnothing)\right)
$$

by "reasoning inside $X_{\alpha+1}$ " (see Remark 11). Let $\Phi \cap X_{\alpha} \uparrow=\varnothing$ and $\Phi \neq \varnothing$. Take $y \in \Phi$ and show that $y \cap \Phi=\varnothing$. Indeed, from $\Phi \cap X_{\alpha} \uparrow=\varnothing$ it follows that $y \notin X_{\alpha} \uparrow$, so $y \subset X_{\alpha} \uparrow$ by (31) and, therefore, $y \cap \Phi \subset X_{\alpha} \uparrow \cap \Phi=\varnothing$.

Suppose now that $\alpha \in$ Lim Ord $^{\bullet}$, and let $X_{\beta}$ be regular outside $X_{0}$ for all $\beta<\alpha$. By Lemma 9, for proving the regularity of $X_{\alpha}$ outside $X_{0}$, it suffices to consider a Booleanvalued class $\Phi \Subset X_{\alpha}$ satisfying $\Phi \leqslant \neg\left(X_{0} \uparrow_{X_{\alpha}}\right)$ and show that $X_{\alpha} \vDash \mu(\Phi)$ or, which is equivalent,

$$
\left(\forall x \in X_{\alpha}\right) X_{\alpha} \vDash(x \in \Phi \Rightarrow(\exists y \in \Phi)(y \cap \Phi=\varnothing)) .
$$

If $x \in X_{\alpha}=\bigcup_{\beta<\alpha} X_{\beta}$ then $x \in X_{\beta}$ for some ordinal $\beta<\alpha$. Prove the validity

$$
X_{\alpha} \vDash(x \in \Phi \Rightarrow(\exists y \in \Phi)(y \cap \Phi=\varnothing))
$$

by "reasoning inside $X_{\alpha}$ ". Relying upon the relations $\Phi \cap X_{0} \uparrow=\varnothing, x \in X_{\beta} \uparrow$, and $x \in \Phi$, show that $(\exists y \in \Phi)(y \cap \Phi=\varnothing)$. Since the class $X_{\beta} \uparrow \backslash X_{0} \uparrow$ is regular (see Lemma 26) and

$$
\varnothing \neq \Phi \cap X_{\beta} \uparrow \subset X_{\beta} \uparrow \backslash X_{0} \uparrow,
$$

there exists $y \in \Phi \cap X_{\beta} \uparrow$ such that $y \cap \Phi \cap X_{\beta} \uparrow=\varnothing$. By the transitivity of $X_{\beta} \uparrow$, from $y \in X_{\beta} \uparrow$ it follows that $y \subset X_{\beta} \uparrow$. Thus, $y \cap \Phi=y \cap \Phi \cap X_{\beta} \uparrow=\varnothing$.

(b): Consider the family of subsystems $X_{\alpha} \subset X\left(\alpha \in \mathrm{Ord}^{\bullet}\right)$ constructed with the use of transfinite recursion by (30) starting from the given subsystem $X_{0} \subset X$.

Induct on $\alpha \in$ Ord $^{\bullet}$ to show that $X_{\alpha} \preccurlyeq X$. The case of $\alpha=0$ is contained in Definition 54 (a). Suppose that $X_{\alpha} \preccurlyeq X$, consider arbitrary $x \in X, y \in X_{\alpha+1}$, and establish the inequality $[x \in y] \leqslant\left[x \in X_{\alpha+1} \uparrow\right]$. If $y \in X_{\alpha}$ then

$$
[x \in y] \leqslant\left[x \in X_{\alpha} \uparrow\right] \leqslant\left[x \in X_{\alpha+1} \uparrow\right] ;
$$

and if $y \in \mathcal{P}_{X}\left(X_{\alpha}\right)$ then $y \simeq P \uparrow, P \subset{ }^{\%} X_{\alpha}$ and so, by Lemma 5 (b),

$$
[x \in y]=[x \in P \uparrow] \leqslant\left[x \in X_{\alpha} \uparrow\right] \leqslant\left[x \in X_{\alpha+1} \uparrow\right] .
$$

If $\alpha \in \operatorname{Lim}$ Ord ${ }^{\bullet}$ and $X_{\beta} \preccurlyeq X$ for all $\beta<\alpha$, then $X_{\alpha}=\bigcup_{\alpha<\beta} X_{\beta} \preccurlyeq X$ by Proposition 19(c).

By Proposition 19(a), the above implies that $X_{\alpha} \preccurlyeq X_{\alpha+1}$ for all $\alpha \in$ Ord, which corresponds to Definition 52 (a). The extensionality of $X_{\alpha+1}$ (Definition 52 (b)) follows from Corollary 11 due to the extensionality of $X$ and the transitivity $X_{\alpha+1} \preccurlyeq X$ established above. Conditions (c) and (d) of Definition 52 are ensured by the definition of $X_{\alpha+1}$; and Definition 52 (e) follows from Definition 54 (e). Thus, the family $\left(X_{\alpha}\right)_{\alpha \in \mathrm{Ord}} \bullet$ is an intensional hierarchy.

For proving the equality $X=X_{\infty}$, we will need several auxiliary facts. Show that

$$
(\forall x \in X)\left(\exists y \in X_{\infty}\right)[x=y]=\left[x \subset X_{\infty} \uparrow\right] .
$$


In the case of $x \in X_{0}$, (32) is obvious. Let $x \notin X_{0}$. By Definition 54(d) the element $x \in X$ is predicative, and so by Lemma 8 there is a set $P \subset{ }^{\%} X_{\infty}$ such that $\left[x \subset X_{\infty} \uparrow\right]=[x=P \uparrow]$. By (a), the system $X_{\infty}$ satisfies Definition 54(c); therefore, the Boolean-valued class $P \uparrow$ is represented by some $y \in X_{\infty}$, which is desired.

Show also that

$$
(\forall x \in X)\left[x \in X_{\infty} \uparrow\right]=\left[x \subset X_{\infty} \uparrow\right] .
$$

The inequality " $\leqslant$ " is guaranteed by the transitivity $X_{\infty} \preccurlyeq X$ proven above. On the other hand, by (32), for every $x \in X$ there is $y \in X_{\infty}$ satisfying $[x=y]=\left[x \subset X_{\infty} \uparrow\right]$, and so

$$
\left[x \subset X_{\infty} \uparrow\right]=[x=y] \wedge\left[y \in X_{\infty} \uparrow\right] \leqslant\left[x \in X_{\infty} \uparrow\right] .
$$

Passing to the proof of the equality $X=X_{\infty}$, assume on the contrary that there exists $x \in X$ not belonging to $X_{\infty}$. Put $b:=\left[x \notin X_{\infty} \uparrow\right]=\left[x \not \subset X_{\infty} \uparrow\right]$ (see (33)). Note that $b \neq 0_{B}$. Indeed, if $b=0_{B}$ then $X \vDash\left(x \subset X_{\infty} \uparrow\right)$ and then, by (32), there exists $y \in X_{\infty}$ such that $[x=y]=\left[x \subset X_{\infty} \uparrow\right]=1_{B}$, that is, $x \simeq y$, which, with account taken of Definition $54(\mathrm{e})$, implies that $x=y$, and so $x \in X_{\infty}$. Let $\Phi:=\neg\left(X_{\infty} \uparrow\right)$ be the complement to $X_{\infty} \uparrow$ inside $X$. Since $X$ is regular outside $X_{0}$ (see Definition $54(\mathrm{f})$ ), $\Phi \leqslant \neg\left(X_{0} \uparrow\right)$ and $[\Phi \neq \varnothing] \geqslant b$ imply $[(\exists y \in \Phi)(y \cap \Phi=\varnothing)] \geqslant b$; therefore, $[y \in \Phi \wedge y \cap \Phi=\varnothing] \neq 0_{B}$ for some $y \in X$. On the other hand, by (33),

$$
[y \in \Phi \wedge y \cap \Phi=\varnothing]=\left[y \notin X_{\infty} \uparrow \wedge y \subset X_{\infty} \uparrow\right]=0_{B} .
$$

The uniqueness of the intensional hierarchy in (b) follows from Lemma 32 (a). Indeed, if $\left(X_{\alpha}\right)_{\alpha \in \text { Ord }} \bullet$ and $\left(Y_{\alpha}\right)_{\alpha \in \text { Ord }} \bullet$ are intensional hierarchies such that $X_{0}=Y_{0}$ and $X_{\infty}=Y_{\infty}$ then, for all $\alpha \in$ Ord $^{\bullet}$, we have $\left.\left(\operatorname{id}_{X_{\infty}}\right)\right|_{X_{\alpha}}: X_{\alpha} \leftrightarrow_{B} Y_{\alpha}$, that is, $X_{\alpha}=Y_{\alpha}$.

The assertion below follows from Lemmas 31 and 32 (c) and Theorem 13.

\section{Theorem 14.}

(a) For every extensional B-system $Y$, there exists a B-valued universe $X$ over $X_{0}$ such that $X_{0} \leftrightarrow_{B} Y$; moreover, such an $X$ is unique up to isomorphism.

(b) If $X$ and $Y$ are B-valued universes over $X_{0}$ and $Y_{0}$ then each isomorphism $f_{0}: X_{0} \leftrightarrow_{B} Y_{0}$ extends to a unique isomorphism $f: X \leftrightarrow_{B} Y$.

Applying Theorem 14(a) to some extensional $B$-system $Y$ and considering a $B$-valued universe $X$ over a copy $X_{0}$ of the system $Y$, agree to identify $X_{0}$ with $Y$ and refer to $X$ as a Boolean-valued universe over $Y$.

\subsection{The Boolean-Valued Universe}

In the present subsection, we formulate the defining properties of the classical Booleanvalued universe $\mathbb{V}^{(B)}$ as an algebraic system, prove the existence of such a system and its uniqueness up to a unique isomorphism. These well-known facts (see $[1,5])$ are reproduced here as direct consequences of the general properties of a Boolean-valued universe over an arbitrary extensional system which are established in Section 4.1. The new results presented in the subsection include examples of Boolean-valued systems with unusual combinations of properties. The examples show that, for each complete Boolean algebra $B$, none of the conditions listed in the definition of Boolean-valued universe follows from the other conditions.

Definition 55. A B-valued system $X$ is called a Boolean-valued (more exactly, B-valued) universe if $X$ satisfies the following conditions (see [1] (3.4)):
(a) $X$ is extensional:
$X \vDash(\forall x, y)((\forall z)(z \in x \Leftrightarrow z \in y) \Rightarrow x=y) ;$
(b) $X$ is intensional:
$\left(\forall P \subset{ }^{\%} X\right)(\exists x \in X)(x \simeq P \uparrow) ;$
(c) $X$ is predicative:
$(\forall x \in X)\left(\exists P \subset{ }^{\%} X\right)(x \simeq P \uparrow)$;
(d) $X$ is separated:
$(\forall x, y \in X)(X \vDash(x=y) \Rightarrow x=y)$;
(e) $X$ is regular:
$X \vDash(\mathbb{E} \Phi)((\exists y)(y \in \Phi) \Rightarrow(\exists y \in \Phi)(\forall z \in \Phi)(z \notin y))$. 
Recall that, by (b) and (c), the regularity, (e), of $X$ is equivalent to the validity in $X$ of the axiom of regularity; see Proposition 20. Moreover, owing to Corollary 10 (c) and Theorem 9 (c), conditions (a)-(c) imply that the regularity of $X$ is equivalent to its $\sigma$-regularity.

Lemma 33. Let $Y$ be an arbitrary extensional B-system. If a B-system $X$ is a Boolean-valued universe over $Y$ then

(a) $X$ is extensional;

(b) $X$ is intensional;

(c) $X$ is predicative if and only if $Y$ is predicative;

(d) $X$ is separated if and only if $Y$ is separated;

(e) $X$ is regular if and only if $Y$ is regular.

Proof. Assertions (a) and (b) are explicitly contained in Definition 54; (c) follows from Proposition 18 (a) and Definition 54(a); (d) is guaranteed by Definition 54(e); (e) is a consequence of Proposition 21, Corollary 14 and Definition 54(f).

Lemma 33 implies that the notion of $B$-valued universe coincides with the notion of Boolean-valued universe over a predicative separated regular $B$-system. The simplest of these $B$-systems is a singleton regular $B$-system. (It would be even easier to speak of a universe over $\varnothing$ but an algebraic system cannot be empty.)

Corollary 15. The following properties of a B-system $X$ are equivalent:

(a) $X$ is a Boolean-valued universe;

(b) $X$ is a Boolean-valued universe over $\{y\}$, where $y \in X$ is such that $X \vDash(y=\varnothing)$;

(c) $X$ is a Boolean-valued universe over a B-system $Y=\{y\}$ such that $Y \vDash(y \notin y)$.

The next assertion, given (without proof) in [1] (3.4), follows from Theorem 14 and Corollary 15. (The author is indebted to Professor Robert M. Solovay for a discussion on approaches to proving this theorem.)

\section{Theorem 15.}

(a) For every complete Boolean algebra B, there exists a B-valued universe unique up to isomorphism.

(b) For arbitrary B-valued universes $X$ and $Y$, there is a unique isomorphism $f: X \leftrightarrow_{B} Y$.

Definition 56. The B-valued universe, characterized up to isomorphism, is denoted by $\mathbb{V}^{(B)}$; and the corresponding truth values $[\varphi]_{\mathbb{V}(B)}$ are written down as $\llbracket \varphi \rrbracket ;$ see $[1,4,5]$.

As is known, the Boolean-valued universe is a model of ZFC, which fact is called the transfer principle; see [4] (2.4), [5] (4.4). More exactly, there are classes $\mathbb{V}^{(B)}, \llbracket=\rrbracket$, and $\llbracket \in \rrbracket$, defined with parameter $B$, such that

$$
\begin{aligned}
\mathrm{ZFC}, \mathrm{B} \text { is a complete Boolean algebra } \vdash \\
\qquad \begin{array}{l}
\left(\mathbb{V}^{(B)}, \llbracket=\rrbracket, \llbracket \in \rrbracket\right) \text { is a } B \text {-valued universe, } \\
\left(\mathbb{V}^{(B)}, \llbracket=\rrbracket, \llbracket \in \rrbracket\right) \vDash \mathrm{ZFC} .
\end{array}
\end{aligned}
$$

Examples 1-5 below show that, for every complete Boolean algebra $B$, each of the five conditions (a)-(e) listed in Definition 55 of the Boolean-valued universe is essential, that is, none of them follows from the other four conditions. The main tools here are Theorem 14 (a) and Lemma 33.

Example 1. For every complete Boolean algebra B, there exists a B-valued system that is intensional, predicative, separated, and regular but not extensional. 
Proof. Extend ZFC by the definitions of the constants

$$
2_{1}:=\{\{\varnothing\}\}, \quad 2_{2}:=\{\varnothing,\{\varnothing\}\} .
$$

Consider the Boolean-valued universe $\mathbb{V}^{(B)}$ and the elements $\varnothing^{\wedge},\{\varnothing\}^{\wedge}, 2_{1}^{\wedge}, 2_{2}^{\wedge} \in \mathbb{V}^{(B)}$ representing the ascents $\varnothing \uparrow,\left\{\varnothing^{\wedge}\right\} \uparrow,\left\{\{\varnothing\}^{\wedge}\right\} \uparrow$, and $\left\{\varnothing^{\wedge},\{\varnothing\}^{\wedge}\right\} \uparrow$ respectively. As is easy to see, $\mathbb{V}^{(B)} \vDash\left(2_{1}^{\wedge}=2_{1}\right)$ and $\mathbb{V}^{(B)} \vDash\left(2_{2}^{\wedge}=22\right)$ (see Definition 18).

Show that the subsystem

$$
X:=\left\{x \in \mathbb{V}^{(B)}: \mathbb{V}^{(B)} \vDash(x \not \subset\{\varnothing\})\right\} \subset \mathbb{V}^{(B)}
$$

possesses the desired properties.

Since $\mathbb{V}^{(B)} \vDash Z F C$; according to Proposition 9, the theorems $2_{1}, 2_{2} \not \subset\{\varnothing\}$ and $2_{1} \neq 2_{2}$ imply that $2_{1}^{\wedge}, 2_{2}^{\wedge} \in X$ and $\left[2 \wedge=2_{2}^{\wedge}\right]_{X}=\llbracket 2_{1}^{\wedge}=2_{2}^{\wedge} \rrbracket=0_{B}$. On the other hand, for all $x \in X$,

$$
\begin{gathered}
x=\llbracket x \in 2_{1}^{\wedge} \rrbracket=\llbracket x \in 2_{1} \wedge x \not \subset\{\varnothing\} \rrbracket=0_{B} \\
=\llbracket x \in 2_{2} \wedge x \not \subset\{\varnothing\} \rrbracket=\llbracket x \in 2_{2}^{\wedge} \rrbracket=\left[x \in 2_{2}^{\wedge}\right]_{X} .
\end{gathered}
$$

Thus, $X \vDash\left(2_{1}^{\wedge} \neq 2_{2}^{\wedge}\right)$ and $X \vDash(\forall x)\left(x \in 2_{1}^{\wedge} \Leftrightarrow x \in 2_{2}^{\wedge}\right)$, and so the system $X$ is not extensional.

For proving the intensionality of $X$, for an arbitrary subset $P \subset{ }^{\%} X$, consider the element $u \in \mathbb{V}^{(B)}$ representing the ascent $P \uparrow$, put $x:=\left.\left.u\right|_{b} \sqcup 2_{1}^{\wedge}\right|_{\neg b}$ (see Definition 39), where $b:=\bigvee_{p \in P} \Delta p$, and show that $x \in X$ and $x \simeq P \uparrow_{X}$. By Lemma 6 (a), we have

$$
\llbracket P \uparrow \not \subset\{\varnothing\} \rrbracket=\llbracket(\exists y \in P \uparrow)(y \notin\{\varnothing\}) \rrbracket=\bigvee_{p \in P} \llbracket p \notin\{\varnothing\} \rrbracket \geqslant \bigvee_{p \in P} \llbracket p \not \subset\{\varnothing\} \rrbracket=\bigvee_{p \in P} \Delta p=b,
$$

which implies that

$$
\llbracket x \not \subset\{\varnothing\} \rrbracket=(\llbracket u \not \subset\{\varnothing\} \rrbracket \wedge b) \vee\left(\llbracket 2_{1} \not \subset\{\varnothing\} \rrbracket \wedge \neg b\right)=(\llbracket P \uparrow \not \subset\{\varnothing\} \rrbracket \wedge b) \vee \neg b=1_{B} ;
$$

that is, $x \in X$. Moreover, for all $y \in X$, using the equality $\llbracket y \in 2_{1}^{\wedge} \rrbracket=0_{B}$ (see (34)), we have

$$
\begin{aligned}
{[y \in x]_{X} } & =\llbracket y \in x \rrbracket=(\llbracket y \in u \rrbracket \wedge b) \vee\left(\llbracket y \in 2_{1}^{\wedge} \rrbracket \wedge \neg b\right)=\llbracket y \in P \uparrow \rrbracket \wedge b \\
& =\llbracket y \in P \uparrow \wedge P \uparrow \neq \varnothing \rrbracket=\llbracket y \in P \uparrow \rrbracket=P \uparrow(y)=P \uparrow_{X}(y) .
\end{aligned}
$$

The predicativity and separatedness of $X$ are immediate from the predicativity and separatedness of $\mathbb{V}^{(B)}$ because $x \Downarrow_{X} \subset x \Downarrow_{\mathbb{V}^{(B)}}$ and $[x=y]_{X}=\llbracket x=y \rrbracket$ for all $x, y \in X$. The regularity of $X$ follows from that of $\mathbb{V}^{(B)}$ by Proposition 21 .

Example 2. For every complete Boolean algebra B, there exists a B-valued system that is extensional, predicative, separated, and regular but not intensional.

Proof. The extensionality, predicativity, separatedness, and regularity of a one-element system $\{x\}$ with interpretations $[=](x, x)=1_{B}$ and $[\epsilon](x, x)=0_{B}$ are obvious. Since $[x \in x]=0_{B} \neq 1_{B}=\{x\} \uparrow(x)$, the Boolean-valued class $\{x\} \uparrow$ is not represented by the (unique) element $x$; and so the system under consideration is not intensional.

Example 3. For every complete Boolean algebra B, there exists a B-valued system that is extensional, intensional, separated, and regular but not predicative.

Proof. Let $Z$ be an extensional, separated, regular $B$-valued system whose underlying class is a proper class different from the class of all sets. (As $Z$ we can take, for example, the isomorphic copy $\{\varnothing\} \times \mathbb{V}^{(B)}$ of the Boolean-valued universe $\mathbb{V}^{(B)}$.) 
Consider an arbitrary set $\infty$ not belonging to $Z$, put $Y:=Z \cup\{\infty\}$, and extend the Boolean-valued interpretations of $Z$ onto $Y$ by putting

$$
\begin{aligned}
& {[=]_{Y}(\infty, \infty)=1_{B},} \\
& {[\in]_{Y}(\infty, \infty)=[=]_{Y}(z, \infty)=[=]_{Y}(\infty, z)=[\in]_{Y}(\infty, z)=0_{B},} \\
& {[\in]_{Y}(z, \infty)=1_{B}}
\end{aligned}
$$

for all $z \in Z$. An elementary check shows that $Y$ is a $B$-system.

For proving that $Y$ is extensional, consider arbitrary $x, y \in Y$ and show that

$$
[(\forall z)(z \in x \Leftrightarrow z \in y)]_{Y} \leqslant[x=y]_{Y} .
$$

The case of $x, y \in Z$ amounts to the extensionality of $Z$; the case of $x=y=\infty$ is trivial; and in the case of $x \in Z$ and $y=\infty$ the desired inequality is guaranteed by the regularity of $Z$, since the validity $Z \vDash \mu(\{x\} \uparrow)$ (see Definition 47) implies $Z \vDash(x \notin x)$, and so

$$
[(\forall z)(z \in x \Leftrightarrow z \in \infty)]_{Y} \leqslant[x \in x \Leftrightarrow x \in \infty]_{Y}=[x \in x]_{Z}=0_{B}=[x=\infty]_{Y} .
$$

As is easy to see, $Y$ is separated and $Z \preccurlyeq Y$. Since

$$
Y \vDash(\mathbb{V} \Phi)(\Phi \cap Z \uparrow=\varnothing \Rightarrow \Phi \subset\{\infty\}),
$$

the system $Y$ is regular outside $Z$. By Corollary 14 , the regularity of $Z$ implies the regularity of $Y$. The system $Y$ is not predicative since the saturated descent $\infty \Downarrow$ includes the proper class $\infty \downarrow=Z$.

Lemma 33 implies that the Boolean-valued universe over $Y$ satisfies the requirements listed in the statement.

Example 4. For every complete Boolean algebra B, there exists a B-valued system that is extensional, intensional, predicative, and regular but not separated.

Proof. According to Lemma 33, all the properties listed in the above statement are possessed by the Boolean-valued universe over a two-element $B$-system $Y$ with interpretations $[=]_{Y}: Y^{2} \rightarrow\left\{1_{B}\right\}$ and $[\in]_{Y}: Y^{2} \rightarrow\left\{0_{B}\right\}$.

Example 5. For every complete Boolean algebra B, there exists a B-valued system that is extensional, intensional, predicative, and separated but not regular.

Proof. A singleton $Y=\{y\}$ endowed with the interpretations $[=]_{Y}(y, y)=[\in]_{Y}(y, y)=1_{B}$ is an extensional, predicative, separated, and non-regular $B$-system. Therefore, owing to Lemma 33, the Boolean-valued universe over $Y$ possesses the required properties.

\subsection{Hierarchies in the Boolean-Valued Universe}

In this subsection, we propose descriptions of the structure of the Boolean-valued universe $\mathbb{V}^{(B)}$ by means of four hierarchies, one of which reproduces the intensional hierarchy, the second serves as the descent of the von Neumann hierarchy, and the other two are generated by some ascents and mixings.

In accordance with Remark 13, for every subset $P \subset{ }^{\%} \mathbb{V}^{(B)}$, the Boolean-valued class $P \uparrow \Subset \mathbb{V}^{(B)}$ is identified with the element of $\mathbb{V}^{(B)}$ that represents the class.

The hierarchy in the theorem below corresponds to the classical construction of the (unseparated) Boolean-valued universe (see [2] (14.15), [4] (2.1.2), [5] (4.1.2)) and, without the zero term, coincides with the intensional hierarchy (30) over a singleton regular $B$-system. 
Theorem 16. Using transfinite recursion, define the family of subsets $\mathbb{V}_{\alpha}^{(B)} \subset \mathbb{V}^{(B)}(\alpha \in$ Ord $)$ by putting

Then

$$
\begin{array}{ll}
\mathbb{V}_{0}^{(B)}=\varnothing ; & \\
\mathbb{V}_{\alpha+1}^{(B)}=\mathcal{P}_{\mathbb{V}^{(B)}}\left(\mathbb{V}_{\alpha}^{(B)}\right), & \alpha \in \text { Ord; } \\
\mathbb{V}_{\alpha}^{(B)}=\bigcup_{\beta<\alpha} \mathbb{V}_{\beta}^{(B)}, & \alpha \in \text { Lim Ord. }
\end{array}
$$

$$
\mathbb{V}^{(B)}=\bigcup_{\alpha \in \text { Ord }} \mathbb{V}_{\alpha}^{(B)}
$$

Moreover, the family $\left(X_{\alpha}\right)_{\alpha \in \text { Ord }}$ defined by the rule

$$
X_{\alpha}= \begin{cases}\mathbb{V}_{\alpha+1}^{(B)} & \text { for } \alpha<\omega ; \\ \mathbb{V}_{\alpha}^{(B)} & \text { for } \omega \leqslant \alpha<\infty ; \\ \mathbb{V}^{(B)} & \text { for } \alpha=\infty\end{cases}
$$

is an intensional hierarchy over $X_{0}=\mathbb{V}_{1}^{(B)}=\{\varnothing \uparrow\}$. In particular, $\mathbb{V}_{\beta}^{(B)}$ is a transitive subset of $\mathbb{V}_{\alpha}^{(B)}$ for all $\beta \leqslant \alpha \in$ Ord $^{\bullet}$, where $\mathbb{V}_{\infty}^{(B)}:=\mathbb{V}^{(B)}$.

Proof. Inducting on $\alpha \in$ Ord, show that $\mathbb{V}_{\beta}^{(B)} \subset \mathbb{V}_{\alpha}^{(B)}$ for $\beta<\alpha$. Let $\mathbb{V}_{\gamma}^{(B)} \subset \mathbb{V}_{\beta}^{(B)}$ for all $\gamma<\beta<\alpha$. Consider $\beta<\alpha$ and $x \in \mathbb{V}_{\beta}^{(B)}$ and show that $x \in \mathbb{V}_{\alpha}^{(B)}$. The cases of $\alpha=0$ and $\alpha \in \operatorname{Lim}$ Ord are trivial. Let $\alpha=\alpha_{0}+1$. The definition (35) implies that $x \in \mathcal{P}_{\mathbb{V}^{(B)}}\left(\mathbb{V}_{\gamma}^{(B)}\right)$ for some $\gamma<\beta \leqslant$ $\alpha_{0}<\alpha$. By the induction hypothesis, $\mathbb{V}_{\gamma}^{(B)} \subset \mathbb{V}_{\alpha_{0}}^{(B)}$, and so $x \in \mathcal{P}_{\mathbb{V}(B)}\left(\mathbb{V}_{\gamma}^{(B)}\right) \subset \mathcal{P}_{\mathbb{V}(B)}\left(\mathbb{V}_{\alpha_{0}}^{(B)}\right)=\mathbb{V}_{\alpha}^{(B)}$.

The inclusions $X_{\alpha} \subset X_{\alpha+1}$ imply that $X_{\alpha+1}=X_{\alpha} \cup \mathcal{P}_{X_{\infty}}\left(X_{\alpha}\right)$ for all $\alpha \in$ Ord. It remains to use Theorem 13(b).

Corollary 16 ([4] (2.1.3), [5] (4.1.3)). Let $C$ be a subclass of $\mathbb{V}^{(B)}$. If $P \uparrow \in C$ for all subsets $P \subset{ }^{\%} C$, then $C=\mathbb{V}^{(B)}$.

Proof. Suppose that $C \neq \mathbb{V}^{(B)}$. By Theorem 16, there exists a least ordinal $\alpha$ for which there is an element $x \in \mathbb{V}_{\alpha}^{(B)} \backslash C$. From (35) it is clear that $\alpha \neq 0$ and $\alpha \notin$ Lim Ord. On the other hand, if $\alpha=\beta+1$ then $x=P \uparrow$ for some $P \subset{ }^{\%} \mathbb{V}_{\beta}^{(B)}$. Hence, $\mathbb{V}_{\beta}^{(B)} \subset C$ implies $x \in C$.

Definition 57. Since the Boolean-valued universe $\mathbb{V}^{(B)}$ is a model of ZFC, the equality

$$
\mathbb{V}=\bigcup_{\alpha \in \text { Ord }} \mathbb{V}_{\alpha}
$$

is valid in $\mathbb{V}^{(B)}$; that is, inside $\mathbb{V}^{(B)}$, the class of all sets $\mathbb{V}$ coincides with the union of the classical von Neumann cumulative hierarchy $\left(\mathbb{V}_{\alpha}\right)_{\alpha \in \mathrm{Ord}}$ (see (22)). Define the descent of the hierarchy $\left(\mathbb{V}_{\alpha}\right)_{\alpha \in \text { Ord }}$ from $\mathbb{V}^{(B)}$ by assigning to each ordinal $\alpha$ the descent $\mathcal{V}_{\alpha} \downarrow$ of the element $\mathcal{V}_{\alpha} \in \mathbb{V}^{(B)}$ that is equal inside $\mathbb{V}^{(B)}$ to the corresponding term $\mathbb{V}_{\alpha^{\wedge}}$ of the von Neumann hierarchy (see [4] (2.4.10), [5] (4.4.10)):

$$
\mathbb{V}^{(B)} \vDash\left(\mathcal{V}_{\alpha}=\mathbb{V}_{\alpha^{\wedge}}\right)
$$

Remark 17. It would be natural to expect that hierarchy (35), which is a Boolean-valued analog of hierarchy (22), would coincide with the descent of the latter: $\left(\mathbb{V}_{\alpha}^{(B)}\right)_{\alpha \in \text { Ord }}=\left(\mathcal{V}_{\alpha} \downarrow\right)_{\alpha \in \text { Ord }}$. However, this fails for every infinite complete Boolean algebra $B$, since not all subsets $\mathbb{V}_{\alpha}^{(B)} \subset \mathbb{V}^{(B)}$ are cyclic. Indeed, if $\left(d_{n}\right)_{n \in \omega}$ is a partition of unity constituted by nonzero elements $d_{n} \in B$ then the join $\left.\sqcup_{n \in \omega} n^{\wedge}\right|_{d_{n}}$ belongs to $\mathcal{V}_{\omega} \downarrow$ but does not belong to $\mathbb{V}_{\omega}^{(B)}=\bigcup_{n \in \omega} \mathbb{V}_{n}^{(B)}$.

The following assertion shows that, in order to turn (35) into the descent of the von Neumann hierarchy, it suffices to add mixings at the limit steps. 
Theorem 17. Using transfinite induction, define the family of subsets $\mathbb{U}_{\alpha}^{(B)} \subset \mathbb{V}^{(B)}(\alpha \in$ Ord $)$ by putting

$$
\begin{aligned}
& \mathbb{U}_{0}^{(B)}=\varnothing ; \\
& \mathbb{U}_{\alpha+1}^{(B)}=\mathcal{P}_{\mathbb{V}^{(B)}}\left(\mathbb{U}_{\alpha}^{(B)}\right), \quad \alpha \in \text { Ord; } \\
& \mathbb{U}_{\alpha}^{(B)}=\operatorname{mix} \bigcup_{\beta<\alpha} \mathbb{U}_{\beta}^{(B)}, \quad \alpha \in \text { Lim Ord. }
\end{aligned}
$$

Then $\left(\mathbb{U}_{\alpha}^{(B)}\right)_{\alpha \in \text { Ord }}$ is the descent of the von Neumann hierarchy $\left(\mathbb{V}_{\alpha}\right)_{\alpha \in \text { Ord }}$ from $\mathbb{V}^{(B)}$; that is,

$$
\mathbb{U}_{\alpha}^{(B)}=\mathcal{V}_{\alpha} \downarrow \text { for all } \alpha \in \text { Ord, }
$$

where $\mathcal{V}_{\alpha}$ are the elements of $\mathbb{V}^{(B)}$ satisfying $\mathbb{V}^{(B)} \vDash\left(\mathcal{V}_{\alpha}=\mathbb{V}_{\alpha^{\wedge}}\right)$.

Proof. Prove that $\mathbb{U}_{\alpha}^{(B)}=\mathcal{V}_{\alpha} \downarrow$ by induction on $\alpha \in$ Ord.

The induction base $\alpha=0$ is trivial: $\mathbb{U}_{0}^{(B)}=\varnothing=\varnothing \uparrow \downarrow=\mathcal{V}_{0} \downarrow$.

If $\mathbb{U}_{\alpha}^{(B)}=\mathcal{V}_{\alpha} \downarrow$ then, by Proposition 16(a), we have $\mathbb{U}_{\alpha}^{(B)} \uparrow=\mathcal{V}_{\alpha} \downarrow \uparrow=\mathcal{V}_{\alpha}$. Using the relation

$$
\mathbb{V}^{(B)} \vDash\left(\mathcal{P}\left(\mathbb{U}_{\alpha}^{(B)} \uparrow\right)=\mathcal{P}\left(\mathcal{V}_{\alpha}\right)=\mathcal{P}\left(\mathbb{V}_{\alpha^{\wedge}}\right)=\mathbb{V}_{\alpha^{\wedge}+1}=\mathbb{V}_{(\alpha+1)^{\wedge}}=\mathcal{V}_{\alpha+1}\right)
$$

and employing assertions (b) and (c) of Lemma 5, we conclude that, for all $x \in \mathbb{V}^{(B)}$,

$$
\begin{gathered}
x \in \mathbb{U}_{\alpha+1}^{(B)} \Leftrightarrow x \in \mathcal{P}_{\mathbb{V}^{(B)}}\left(\mathbb{U}_{\alpha}^{(B)}\right) \Leftrightarrow\left(\exists P \subset{ }^{\%} \mathbb{U}_{\alpha}^{(B)}\right)(x=P \uparrow) \Leftrightarrow \mathbb{V}^{(B)} \vDash\left(x \subset \mathbb{U}_{\alpha}^{(B)} \uparrow\right) \\
\Leftrightarrow \mathbb{V}^{(B)} \vDash\left(x \in \mathcal{P}\left(\mathbb{U}_{\alpha}^{(B)} \uparrow\right)\right) \Leftrightarrow \mathbb{V}^{(B)} \vDash\left(x \in \mathcal{V}_{\alpha+1}\right) \Leftrightarrow x \in \mathcal{V}_{\alpha+1} \downarrow .
\end{gathered}
$$

If $\alpha \in \operatorname{Lim}$ Ord and $\mathbb{U}_{\beta}^{(B)}=\mathcal{V}_{\beta} \downarrow$ for all $\beta<\alpha$; then, by the equality $\alpha^{\wedge}=\left\{\beta^{\wedge}: \beta \in \alpha\right\} \uparrow$, Corollary 2, Lemma 15, Proposition 16 (a) and [4] (3.1.9(1)) or [5] (5.1.7(1)), for all $x \in \mathbb{V}^{(B)}$ we have

$$
\begin{aligned}
& x \in \mathbb{U}_{\alpha}^{(B)} \Leftrightarrow x \in \operatorname{mix} \bigcup_{\beta<\alpha} \mathbb{U}_{\beta}^{(B)} \Leftrightarrow \vee\left\{\llbracket x=y \rrbracket: y \in \bigcup_{\beta<\alpha} \mathbb{U}_{\beta}^{(B)}\right\}=1_{B} \\
& \Leftrightarrow \bigvee_{\beta<\alpha} \bigvee_{y \in \mathbb{U}_{\beta}^{(B)}} \llbracket x=y \rrbracket=1_{B} \Leftrightarrow \bigvee_{\beta<\alpha}^{\bigvee \llbracket x \in \mathbb{U}_{\beta}^{(B)} \uparrow \rrbracket=1_{B}} \\
& \Leftrightarrow \bigvee_{\beta<\alpha} \llbracket x \in \mathcal{V}_{\beta} \downarrow \uparrow \rrbracket=1_{B} \Leftrightarrow \bigvee_{\beta<\alpha}^{\bigvee} \llbracket x \in \mathcal{V}_{\beta} \rrbracket=1_{B} \Leftrightarrow \bigvee \underset{\beta \in \alpha}{V} \llbracket x \in \mathbb{V}_{\beta^{\wedge}} \rrbracket=1_{B} \\
& \Leftrightarrow \mathbb{V}^{(B)} \vDash\left(\exists \beta \in \alpha^{\wedge}\right)\left(x \in \mathbb{V}_{\beta}\right) \Leftrightarrow \mathbb{V}^{(B)} \vDash\left(\begin{array}{c}
\left.x \in \bigcup_{\beta<\alpha^{\wedge}} \mathbb{V}_{\beta}\right) \\
\end{array}\right. \\
& \Leftrightarrow \mathbb{V}^{(B)} \vDash\left(x \in \mathbb{V}_{\alpha^{\wedge}}\right) \Leftrightarrow \mathbb{V}^{(B)} \vDash\left(x \in \mathcal{V}_{\alpha}\right) \Leftrightarrow x \in \mathcal{V}_{\alpha \downarrow} .
\end{aligned}
$$

The construction of the hierarchy (35) involves the ascents $P \uparrow$ of arbitrary sets $P$ of partial elements:

$$
\mathbb{V}_{\alpha+1}^{(B)}=\mathcal{P}_{\mathbb{V}^{(B)}}\left(\mathbb{V}_{\alpha}^{(B)}\right)=\left\{P \uparrow: P \subset{ }^{\%} \mathbb{V}_{\alpha}^{(B)}\right\} .
$$

On the other hand, by Corollary 8 (b), each element in the Boolean-valued universe is represented as the ascent $\left(\left.Y\right|_{b}\right) \uparrow$ of the set $\left.Y\right|_{b}$ of partial elements with equal domains. In this connection, it seems natural to conjecture that the Boolean-valued universe can be constructed into the hierarchy of ascents of the form $\left(\left.Y\right|_{b}\right) \uparrow$. Nevertheless, the fact below refutes this conjecture. 
Theorem 18. Suppose that a separated extensional B-system $X$ satisfies the ascent principle. Using transfinite induction, define the family of subsets $Y_{\alpha} \subset X$ ( $\alpha \in$ Ord) by putting

$$
Y_{\alpha}=\left\{\left(\left.Y\right|_{b}\right) \uparrow: Y \subset \bigcup_{\beta<\alpha} Y_{\beta}, b \in B\right\}, \quad \alpha \in \text { Ord. }
$$

If the Boolean algebra $B$ is infinite then $\bigcup_{\alpha \in \text { Ord }} Y_{\alpha} \neq X$.

Proof. Define recursively the sequence $\left(y_{n}\right)_{n \in \omega} \subset X$ as follows:

$$
y_{0}:=\varnothing \uparrow, \quad y_{n+1}:=\left\{y_{n}\right\} \uparrow, \quad n \in \omega .
$$

Fix an arbitrary antichain $\left(d_{n}\right)_{n \in \omega}$ of nonzero elements in $B$ and consider the ascents

$$
x_{i}:=\left\{\left.y_{n}\right|_{d_{n+i}}: n \in \omega\right\} \uparrow, \quad i \in \omega .
$$

Since $(\forall n \in \omega) y_{n+1}=\left\{y_{n}\right\} \uparrow$ and $x_{i}=\left(\left.\bigcup_{n \in \omega}\left\{y_{n}\right\}\right|_{d_{n+i}}\right) \uparrow ;$ by Lemma 22, we have

$$
x_{i}=\left.\operatorname{ext} \bigsqcup_{n \in \omega} y_{n+1}\right|_{d_{n+i}}, \quad i \in \omega .
$$

For completing the proof, it suffices to show that $x_{i} \notin Y_{\alpha}$ for all $\alpha \in$ Ord, $i \in \omega$. Induct transfinitely on $\alpha$. Consider $\alpha \in$ Ord, suppose that $x_{i} \notin Y_{\beta}$ for all $\beta<\alpha, i \in \omega$, and assume that $x_{i} \in Y_{\alpha}$ for some $i \in \omega$. Then $x_{i}=\left(\left.Y\right|_{b}\right) \uparrow$, where $Y \subset \cup_{\beta<\alpha} Y_{\beta}, b \in B$. Moreover,

$$
\left[y \in x_{i}\right]=\left[x_{i} \neq \varnothing\right]=\bigvee_{n \in \omega} d_{n+i}=b
$$

for all $y \in Y$ (see Corollary $8(b))$. Since $\left[x_{i} \neq \varnothing\right] \neq 0_{B}$, the set $Y$ is nonempty, and so there exist $\beta<\alpha$ and $y \in Y_{\beta}$ such that $\left[y \in x_{i}\right]=b$.

Reckoning with (37), we have

$$
\bigvee_{n \in \omega} d_{n+i}=\left[y \in x_{i}\right]=\bigvee_{n \in \omega}\left[y=y_{n}\right] \wedge d_{n+i}
$$

which, by Lemma 10 (a), implies that $d_{n+i}=\left[y=y_{n}\right] \wedge d_{n+i}$, that is, $\left[y=y_{n}\right] \geqslant d_{n+i}$ for all $n \in \omega$, and so $\left.y\right|_{b}=\left.\bigsqcup_{n \in \omega} y_{n}\right|_{d_{n+i}}$. Using the equality $y_{0}=\varnothing \uparrow$ and (38), we conclude that

$$
\left.\operatorname{ext} y\right|_{b}=\left.\operatorname{ext} \bigsqcup_{n \in \omega} y_{n+1}\right|_{d_{(n+1)+i}}=\left.\operatorname{ext} \bigsqcup_{n \in \omega} y_{n+1}\right|_{d_{n+(i+1)}}=x_{i+1}
$$

Since $y \in Y_{\beta}$, there are $Z \subset \cup_{\gamma<\beta} Y_{\gamma}$ and $c \in B$ such that $y=\left(\left.Z\right|_{c}\right) \uparrow$. Then, for all $x \in X$,

$$
\begin{gathered}
{\left[\left.x \in \operatorname{ext} y\right|_{b}\right]=\left[\left.x \in y\right|_{b}\right]=[x \in y] \wedge b=\left[x \in\left(\left.Z\right|_{c}\right) \uparrow\right] \wedge b} \\
=\bigvee_{z \in Z}\left[x=\left.z\right|_{c}\right] \wedge b=\bigvee_{z \in Z}\left[x=\left.z\right|_{c \wedge b}\right]=\left[x \in\left(\left.Z\right|_{c \wedge b}\right) \uparrow\right]
\end{gathered}
$$

which, according to (39), implies $x_{i+1}=\left.\operatorname{ext} y\right|_{b}=\left(\left.Z\right|_{c \wedge b}\right) \uparrow \in Y_{\beta}$ contrary to the induction hypothesis.

Lemma 34. If $\left(Z_{i}\right)_{i \in I}$ is a family of subsets of $\mathbb{V}^{(B)},\left(d_{i}\right)_{i \in I} \subset B$ is a partition of unity, and $\left(b_{i}\right)_{i \in I} \subset B$, then there exist $Y \subset \operatorname{mix} \bigcup_{i \in I} Z_{i}$ and $b \in B$ such that

$$
\left.\bigsqcup_{i \in I}\left(\left.Z_{i}\right|_{b_{i}}\right) \uparrow\right|_{d_{i}}=\left(\left.Y\right|_{b}\right) \uparrow .
$$


Proof. Put $Z=\bigcup_{i \in I} Z_{i} \subset \mathbb{V}^{(B)}$ and $P=\left.\bigcup_{i \in I} Z_{i}\right|_{b_{i} \wedge d_{i}} \subset{ }^{\%} Z$. According to Lemma 22, we have $\left.\bigsqcup_{i \in I}\left(\left.Z_{i}\right|_{b_{i}}\right) \uparrow\right|_{d_{i}}=P \uparrow$. By Corollary 8 (a), there are $Y \subset \operatorname{mix} Z$ and $b \in B$ such that $P \uparrow=\left(\left.Y\right|_{b}\right) \uparrow$.

According to Theorem 18, for constructing the Boolean-valued universe, the ascents with constant domain are not enough. The results below show that the situation will change if we add mixings at the limit steps of the hierarchy.

Theorem 19. Using transfinite recursion, define the family of subsets $Y_{\alpha} \subset \mathbb{V}^{(B)}(\alpha \in$ Ord) by putting

Then

$$
\begin{array}{ll}
Y_{0}=\varnothing ; & \\
Y_{\alpha+1}=\left\{\left(\left.Y\right|_{b}\right) \uparrow: Y \subset Y_{\alpha}, b \in B\right\}, & \alpha \in \text { Ord; } \\
Y_{\alpha}=\operatorname{mix} \bigcup_{\beta<\alpha} Y_{\beta}, & \alpha \in \text { Lim Ord. }
\end{array}
$$

(a) $Y_{\alpha} \subset Y_{\beta}$ for $\alpha \leqslant \beta$;

(b) $Y_{\alpha}$ are cyclic for all $\alpha \in$ Ord;

(c) $\mathbb{V}_{\alpha}^{(B)} \subset Y_{\alpha+1}$ for all $\alpha \in$ Ord;

(d) $\mathbb{V}^{(B)}=\bigcup_{\alpha \in \text { Ord }} Y_{\alpha}$.

Proof. (a): Prove that $Y_{\alpha} \subset Y_{\beta}$ for all $\alpha \leqslant \beta$ by induction on $\beta$. Let $\beta \in$ Ord, and let

$$
Y_{\alpha_{1}} \subset Y_{\alpha_{2}} \text { for all } \alpha_{1} \leqslant \alpha_{2}<\beta \text {. }
$$

Consider an arbitrary ordinal $\alpha \leqslant \beta$ and prove that $Y_{\alpha} \subset Y_{\beta}$. This inclusion is obvious if $\alpha=0, \alpha=\beta$, or $\beta \in \operatorname{Lim}$ Ord. Therefore, we will assume that $0<\alpha<\beta$ and $\beta=\beta_{0}+1$ for some $\beta_{0} \in$ Ord. Fix an arbitrary element $x \in Y_{\alpha}$ and show that $x \in Y_{\beta}$.

If $\alpha=\alpha_{0}+1$ for some $\alpha_{0} \in$ Ord then $x=\left(\left.Y\right|_{b}\right) \uparrow$, where $Y \subset Y_{\alpha_{0}}, b \in B$. Since $\alpha_{0}<\beta_{0}<\beta$, by (41) we have $Y_{\alpha_{0}} \subset Y_{\beta_{0}}$; so $Y \subset Y_{\beta_{0}}$ and, therefore, $x=\left(\left.Y\right|_{b}\right) \uparrow \in Y_{\beta_{0}+1}=Y_{\beta}$.

Now, let $\alpha \in \operatorname{Lim}$ Ord. Then $Y_{\alpha}=\operatorname{mix} \bigcup_{\gamma<\alpha} Y_{\gamma}$, and so $x=\left.\bigsqcup_{i \in I} x_{i}\right|_{d_{i}}$, where $\left(d_{i}\right)_{i \in I} \subset B$ is a partition of unity and $x_{i} \in Y_{\gamma_{i}}, \gamma_{i}<\alpha(i \in I)$. Since $\alpha$ is a limit ordinal, for all $i \in I$ we have $\gamma_{i}+1<\alpha$ and, in particular, $\gamma_{i}<\gamma_{i}+1<\beta$, which, owing to (41), implies that $Y_{\gamma_{i}} \subset Y_{\gamma_{i}+1}$. Thus, $x_{i} \in Y_{\gamma_{i}+1}$, and so $x_{i}=\left(Z_{i} \mid b_{i}\right) \uparrow$ for some $Z_{i} \subset Y_{\gamma_{i}}, b_{i} \in B(i \in I)$, and so $x=\left.\bigsqcup_{i \in I}\left(\left.Z_{i}\right|_{b_{i}}\right) \uparrow\right|_{d_{i}}$. By Lemma 34, there exist $Y \subset \operatorname{mix} \bigcup_{i \in I} Z_{i}$ and $b \in B$, such that $x=\left(\left.Y\right|_{b}\right) \uparrow$. Note that

$$
\operatorname{mix} \bigcup_{i \in I} Z_{i} \subset \operatorname{mix} \bigcup_{i \in I} Y_{\gamma_{i}} \subset \operatorname{mix} \bigcup_{\gamma<\alpha} Y_{\gamma}=Y_{\alpha}
$$

and, moreover, by (41), $\alpha \leqslant \beta_{0}<\beta$ implies $Y_{\alpha} \subset Y_{\beta_{0}}$. Hence, $Y \subset Y_{\beta_{0}}$ and, consequently, $x=\left(\left.Y\right|_{b}\right) \uparrow \in Y_{\beta_{0}+1}=Y_{\beta}$.

(b): Applying induction, consider an arbitrary ordinal $\alpha$, suppose that $\operatorname{mix} Y_{\beta}=Y_{\beta}$ for all $\beta<\alpha$, and establish the equality $\operatorname{mix} Y_{\alpha}=Y_{\alpha}$. In the case of $\alpha=0$ or $\alpha \in \operatorname{Lim}$ Ord, the equality is obvious. Assume that $\alpha=\beta+1$ for some $\beta \in$ Ord. Consider an arbitrary element $x \in \operatorname{mix} Y_{\alpha}$ and show that $x \in Y_{\alpha}$. Since

$$
x \in \operatorname{mix} Y_{\beta+1}=\operatorname{mix}\left\{\left(\left.Z\right|_{b}\right) \uparrow: Z \subset Y_{\beta}, b \in B\right\},
$$

there exist a partition of unity $\left(d_{i}\right)_{i \in I} \subset B$ and families $Z_{i} \subset Y_{\beta}, b_{i} \in B(i \in I)$ such that $x=\left.\bigsqcup_{i \in I}\left(\left.Z_{i}\right|_{b_{i}}\right) \uparrow\right|_{d_{i}}$. Lemma 34 implies the representation $x=\left(\left.Y\right|_{b}\right) \uparrow$ for some $Y \subset \operatorname{mix} \bigcup_{i \in I} Z_{i}$ and $b \in B$. By the induction hypothesis, $\operatorname{mix} \bigcup_{i \in I} Z_{i} \subset \operatorname{mix} Y_{\beta}=Y_{\beta}$, and so $Y \subset Y_{\beta}$; thus, $x=\left(\left.Y\right|_{b}\right) \uparrow \in Y_{\beta+1}=Y_{\alpha}$.

(c): Again proceeding by induction, fix an ordinal $\alpha$, suppose that $\mathbb{V}_{\beta}^{(B)} \subset Y_{\beta+1}$ for all $\beta<\alpha$, consider an arbitrary element $x \in \mathbb{V}_{\alpha}^{(B)}$, and show that $x \in Y_{\alpha+1}$. From (35) it follows that $x=P \uparrow$ for some subset $P \subset{ }^{*} \mathbb{V}_{\beta}^{(B)}$, where $\beta<\alpha$. By Corollary 8 (a), there 
are $Y \subset \operatorname{mix} \mathbb{V}_{\beta}^{(B)}$ and $b \in B$ such that $x=\left(\left.Y\right|_{b}\right) \uparrow$. By the induction hypothesis, $\mathbb{V}_{\beta}^{(B)} \subset Y_{\beta+1}$. Moreover, (a) implies that $Y_{\beta+1} \subset Y_{\alpha}$. Thus, $\mathbb{V}_{\beta}^{(B)} \subset Y_{\alpha}$, from which, in view of (b), it follows that $Y \subset \operatorname{mix} \mathbb{V}_{\beta}^{(B)} \subset \operatorname{mix} Y_{\alpha}=Y_{\alpha}$, and so $x=\left(\left.Y\right|_{b}\right) \uparrow \in Y_{\alpha+1}$.

(d): This is a consequence of (c) and (36).

The following assertion shows that equality (d) remains valid if, at the discrete steps of the hierarchy (40), we confine ourselves to ascents of the sets of everywhere defined elements.

Theorem 20. Using transfinite recursion, define the family of subsets $Z_{\alpha} \subset \mathbb{V}^{(B)}(\alpha \in$ Ord) by putting

$$
\begin{array}{ll}
Z_{0}=\varnothing ; & \\
Z_{\alpha+1}=\left\{Z \uparrow: Z \subset Z_{\alpha}\right\}, & \alpha \in \text { Ord; } \\
Z_{\alpha}=\operatorname{mix} \bigcup_{\beta<\alpha} Z_{\beta}, & \alpha \in \text { Lim Ord. }
\end{array}
$$

Then

$$
\mathbb{V}^{(B)}=\bigcup_{\alpha \in \text { Ord }} Z_{\alpha}
$$

Proof. Define the function $\delta:$ Ord $\rightarrow$ Ord by the recursive formula

$$
\delta(\alpha)=\vee\{\delta(\beta): \beta<\alpha\}+\omega, \quad \alpha \in \text { Ord }
$$

By Theorem 19(d), it suffices to prove that

$$
(\forall \alpha \in \operatorname{Ord})\left(Y_{\alpha} \subset Z_{\delta(\alpha)}\right),
$$

where $Y_{\alpha}$ are defined in (40). Inducting on $\alpha \in$ Ord, assume that $Y_{\beta} \subset Z_{\delta(\beta)}$ for all $\beta<\alpha$, and establish the inclusion $Y_{\alpha} \subset Z_{\delta(\alpha)}$.

Let $\alpha=\beta+1$. The obvious monotonicity of $\delta$ implies that

$$
\delta(\alpha)=\vee\{\delta(\gamma): \gamma<\alpha\}+\omega=\vee\{\delta(\gamma): \gamma \leqslant \beta\}+\omega=\delta(\beta)+\omega .
$$

Consider an arbitrary $x \in Y_{\alpha}=Y_{\beta+1}$. By (40), there are $Y \subset Y_{\beta}$ and $b \in B$ such that $x=\left(\left.Y\right|_{b}\right) \uparrow$. Then, for all $z \in \mathbb{V}^{(B)}$,

$$
\begin{aligned}
\llbracket z \in x \rrbracket & =\llbracket z \in\left(\left.Y\right|_{b}\right) \uparrow \rrbracket=\bigvee_{y \in Y} \llbracket z=\left.y\right|_{b} \rrbracket=\bigvee_{y \in Y} \llbracket z=y \rrbracket \wedge b \\
& =\llbracket z \in Y \uparrow \rrbracket \wedge b=\left.\llbracket z \in(Y \uparrow)\right|_{b} \rrbracket=\left.\llbracket z \in(Y \uparrow)\right|_{b} \rrbracket \vee 0_{B} \\
& =\left.\left.\llbracket z \in(Y \uparrow)\right|_{b} \rrbracket \vee \llbracket z \in(\varnothing \uparrow)\right|_{\neg b} \rrbracket,
\end{aligned}
$$

which, by Lemma 12(d), implies that $x=\left.\left.(Y \uparrow)\right|_{b} \sqcup \varnothing \uparrow\right|_{\neg b}$. The inclusions $Y, \varnothing \subset Y_{\beta} \subset Z_{\delta(\beta)}$ imply $Y \uparrow, \varnothing \uparrow \in Z_{\delta(\beta)+1}$, and so

$$
x=\left.\left.(Y \uparrow)\right|_{b} \sqcup \varnothing \uparrow\right|_{\neg b} \in \operatorname{mix} Z_{\delta(\beta)+1} \subset \operatorname{mix} \bigcup_{\gamma<\delta(\beta)+\omega} Z_{\gamma}=Z_{\delta(\beta)+\omega}=Z_{\delta(\alpha)} .
$$

If $\alpha \in \operatorname{Lim}$ Ord then

$$
Y_{\alpha}=\operatorname{mix} \bigcup_{\beta<\alpha} Y_{\beta} \subset \operatorname{mix} \bigcup_{\beta<\alpha} Z_{\delta(\beta)} \subset \operatorname{mix} \bigcup_{\gamma<\delta(\alpha)} Z_{\gamma}=Z_{\delta(\alpha)} .
$$

Corollary 17. If $C$ is a cyclic subclass of $\mathbb{V}^{(B)}$ and $Y \uparrow \in C$ for all subsets $Y \subset C$, then $C=\mathbb{V}^{(B)}$.

Proof. Suppose that $C \neq \mathbb{V}^{(B)}$. By Theorem 20, there exists a least ordinal $\alpha$ for which there is an element $x \in Z_{\alpha} \backslash C$. If $\alpha=\beta+1$ then $x=Y \uparrow$ for some subset $Y \subset Z_{\beta}$, and so $Z_{\beta} \subset C$ 
implies $x \in C$. If $\alpha \in$ Lim Ord then $x=\sqcup P$ for some maximal antichain $P \subset{ }^{\%}\left(\bigcup_{\beta<\alpha} Z_{\beta}\right)$, and then $\bigcup_{\beta<\alpha} Z_{\beta} \subset C$ implies $x \in \operatorname{mix} C=C$.

\section{Applications of the Lévy Hierarchy}

As another application of the formalism of eliminable extensions, we will present examples of using a technique based on the quantifier hierarchy of formulas and terms. In certain cases, the technique provides a simple replacement for the direct calculation of Boolean truth values. With the help of the tool, we analyze the logical structure of several classical definitions of the field of reals and find out which of them guarantee the inclusion $\mathbb{R}^{\wedge} \subset \mathbb{R}$ inside $\mathbb{V}^{(B)}$ for all $B$.

\subsection{Classification of Formulas and Terms}

The hierarchy of $\Sigma_{n}$ and $\Pi_{n}$ formulas suggested by A. Lévy [10] became an actively employed tool in Boolean-valued analysis due to the fact that the relations expressed by $\Sigma_{1}$ formulas are preserved by the canonical embedding in the Boolean-valued universe (see Theorem 21). Having become convinced that an assertion $\varphi\left(x_{1}, \ldots, x_{n}\right)$ is equivalent to a $\Sigma_{1}$ formula, we thereby relieve ourselves of the need to calculate the Boolean truth value of $\varphi\left(x_{1}^{\wedge}, \ldots, x_{n}^{\wedge}\right)$. In this respect, various syntactic methods are of use which help to successively build more and more complex formulas and terms, while staying within the class of $\Sigma_{1}$ constructions. Below, we suggest some additions to the set of such tools.

In what follows, we assume that terms arise within an eliminable extension of ZFC (see Definition 5), and keep the name of ZFC for the extension. In particular, every term $\tau(\ddot{x})$ is associated with a formula $\varphi(\ddot{x}, y)$ of signature $\{=, \in\}$ such that

$$
\mathrm{ZFC} \vdash(y=\tau(\ddot{x}) \Leftrightarrow \varphi(\ddot{x}, y)) .
$$

We also continue to follow Agreement 3 on expressions of the form $\varphi\left(x_{1}, \ldots, x_{n}\right)$ and $\tau\left(x_{1}, \ldots, x_{n}\right)$ and their connection with term substitution.

Definition 58. Let $\mathcal{F}$ be a set of formulas of set-theoretic signature. A formula $\varphi$ is said to belong to class $\mathcal{F}$ and is called an $\mathcal{F}$ formula, whenever ZFC $\vdash\left(\varphi \Leftrightarrow \varphi^{\prime}\right)$ for some $\varphi^{\prime} \in \mathcal{F}$. If ZFC $\vdash(\varphi \Leftrightarrow \psi)$ and $\psi$ is known to be an $\mathcal{F}$ formula, then the equivalence $\varphi \Leftrightarrow \psi$, as well as the formula $\psi$, is called an $\mathcal{F}$ definition for $\varphi$. As is easily seen, every formula admitting an $\mathcal{F}$ definition belongs to class $\mathcal{F}$.

A term $\tau$ belongs to class $\mathcal{F}$ and is called an $\mathcal{F}$ term or an $\mathcal{F}$ definable term, if the equality $y=\tau$ belongs to class $\mathcal{F}$. (Here and in other similar cases, $y$ is implicitly assumed to be a new variable; see Agreement 2. In the present context, the latter amounts to the condition that $y$ is not a parameter of $\tau$.) Therefore, the membership of a term $\tau(\ddot{x})$ in class $\mathcal{F}$ means that

$$
\operatorname{ZFC} \vdash(y=\tau(\ddot{x}) \Leftrightarrow \varphi(\ddot{x}, y))
$$

for some $\mathcal{F}$ formula $\varphi(\ddot{x}, y)$. In this event, the equivalence $y=\tau \Leftrightarrow \varphi$, as well as the formula $\varphi(\tau(\ddot{x}), \ddot{x})$, is called an $\mathcal{F}$ definition for $\tau$.

Definition 59. Let $\Delta_{0}$ be the smallest set of formulas containing the atomic formulas $x \in y$ and closed under the connectives $\vee, \neg,(\exists x \in y)$.

\section{Proposition 22.}

(a) All bounded formulas (see Definition 46) are $\Delta_{0}$.

(b) If $\varphi$ and $\psi$ are $\Delta_{0}$, then so are $\varphi \vee \psi, \varphi \wedge \psi, \neg \varphi, \varphi \Rightarrow \psi, \varphi \Leftrightarrow \psi,(\exists x \in y) \varphi,(\exists ! x \in y) \varphi,(\forall x \in y) \varphi$. 
The following expressions are known to be $\Delta_{0}$ :

$$
\begin{aligned}
& \varnothing ; \quad\{x\} ; \quad\{x, y\} ; \quad(x, y):=\{\{x\},\{x, y\}\} ; \\
& X \cup Y ; \quad X \cap Y ; \quad X \backslash Y ; \cup X ; \quad X \times Y ; \quad X \subset Y ; \\
& f \text { is a function; } \quad f: X \rightarrow Y ; \quad \operatorname{dom} f ; \quad \operatorname{im} f ; \quad f(x) ; \quad x f y:=f((x, y)) ; \\
& 0:=\varnothing ; \quad x+1:=x \cup\{x\} ; \quad \omega=\{0,1,2, \ldots\}:=\text { the least limit ordinal. }
\end{aligned}
$$

The corresponding $\Delta_{0}$ definitions can be found, for example, in [2] (12.10).

Definition 60. Let $\Sigma_{1}$ be the set of all formulas of the form $(\exists x) \varphi$, where $\varphi$ is $\Delta_{0}$.

The following are consequences of [2] (13.10):

\section{Proposition 23.}

(a) All $\Delta_{0}$ formulas and terms are $\Sigma_{1}$.

(b) If $\varphi$ and $\psi$ are $\Sigma_{1}$, then so are $\varphi \vee \psi, \varphi \wedge \psi,(\exists x) \varphi,(\forall x \in y) \varphi$.

(c) If a formula $\varphi$ and a term $\tau$ are $\Sigma_{1}$, then so are the formulas $(\exists x \in \tau) \varphi$ and $(\forall x \in \tau) \varphi$.

(d) If a formula $\varphi(\ddot{x})$ and terms $\dddot{\tau}$ are $\Sigma_{1}$, then so is the formula $\varphi(\ddot{\tau})$.

(e) If terms $\tau(\ddot{x})$ and $\ddot{\sigma}$ are $\Sigma_{1}$, then so is the term $\tau(\ddot{\sigma})$.

Definition 61. A formula $\varphi$ belongs to class $\Delta_{1}$ and is called $a \Delta_{1}$ formula, if $\varphi$ and $\neg \varphi$ are $\Sigma_{1}$. A term $\tau$ belongs to class $\Delta_{1}$ and is called a $\Delta_{1}$ term, if the equality $y=\tau$ is $\Delta_{1}$.

\section{Proposition 24.}

(a) All $\Delta_{1}$ formulas are $\Sigma_{1}$.

(b) The classes of $\Delta_{1}$ terms and $\Sigma_{1}$ terms coincide.

(c) If formulas $\varphi, \psi$ and a term $\tau$ are $\Delta_{1}$, then so are the formulas $\varphi \vee \psi, \varphi \wedge \psi, \neg \varphi, \varphi \Rightarrow \psi, \varphi \Leftrightarrow \psi,(\exists x \in \tau) \varphi,(\exists ! x \in \tau) \varphi,(\forall x \in \tau) \varphi$.

(d) If a formula $\varphi(\ddot{x})$ and terms $\ddot{\tau}$ are $\Delta_{1}$, then so is the formula $\varphi(\ddot{\tau})$.

Proof. Assertion (a) is obvious; (b) follows from the tautology $x \neq \tau \Leftrightarrow(\exists y)(y=\tau \wedge x \neq y)$; (c) for the case in which $\tau$ is a variable, can be found in [2] $(13.10(\mathrm{v}))$; (c) for an arbitrary $\Delta_{1}$ term $\tau$ follows from (d); for justifying (d), it suffices to observe that

$$
\varphi(\dddot{\tau}) \Leftrightarrow(\exists \dddot{y})(\dddot{y}=\dddot{\tau} \wedge \varphi(\dddot{y})), \quad \neg \varphi(\dddot{\tau}) \Leftrightarrow(\exists \dddot{y})(\dddot{y}=\dddot{\tau} \wedge \neg \varphi(\dddot{y})) .
$$

Taking account of the fact that the notions of $\Sigma_{1}$ term and $\Delta_{1}$ term coincide, we choose the latter and speak of $\Delta_{1}$ terms.

Proposition 25. If a formula $\varphi(\ddot{x}, \dddot{y}, \dddot{z})$, a term $\tau(\ddot{x})$ with parameters $\dddot{x}$, and terms $\dddot{\sigma}, \dddot{\rho}$ are $\Delta_{1}$, then so is the term $\{\tau(\ddot{x}): \ddot{x} \in \ddot{\sigma} \wedge \varphi(\ddot{x}, \ddot{\sigma}, \dddot{\rho})\}$.

Proof. According to Proposition $24(\mathrm{~d})$, the formula $\psi:=\varphi(\ddot{x}, \ddot{\sigma}, \ddot{\rho})$ is $\Delta_{1}$. Therefore, the term under consideration admits the following $\Delta_{1}$ definition:

$$
\begin{aligned}
z= & \{\tau(\dddot{x}): \dddot{x} \in \ddot{\sigma} \wedge \psi\} \\
& \Leftrightarrow(\forall y \in z)(\exists \dddot{x} \in \ddot{\sigma})(y=\tau(\dddot{x}) \wedge \psi) \wedge(\forall \ddot{x} \in \ddot{\sigma})(\psi \Rightarrow(\exists y \in z) y=\tau(\ddot{x})) .
\end{aligned}
$$

In Example 6 we will show that the above assertion cannot be expanded to the case of an arbitrary $\Sigma_{1}$ formula $\varphi$. 
Remark 18. In Agreement 4, the default way is described of making conditionally correct definitions correct. Namely, each definition $y=\tau(\ddot{x}) \Leftrightarrow \varphi(\ddot{x}, y)$ that satisfies the correctness $(\exists ! y) \varphi(\ddot{x}, y)$ only under a condition $\delta(\ddot{x})$, is implicitly replaced with the correct definition

$$
y=\tau(\dddot{x}) \Leftrightarrow(\delta(\ddot{x}) \wedge \varphi(\dddot{x}, y)) \vee\left(\neg \delta(\dddot{x}) \wedge y=x_{1}\right) .
$$

Furthermore, if the defining formula $\varphi(\dddot{x}, y)$ is $\Sigma_{1}$ (or $\left.\Delta_{0}\right)$, and the condition $\delta(\ddot{x})$ is $\Delta_{1}$ (or $\Delta_{0}$ ), then the term $\tau(\ddot{x})$ occurs to be $\Delta_{1}$ (respectively, $\Delta_{0}$ ). For instance, the conventional conditionally correct definition "if $f$ is a function and $x \in \operatorname{dom} f$, then $f(x)$ is the only $y$ such that $(x, y) \in f$ " is implicitly replaced with the correct $\Delta_{0}$ definition

$$
y=f(x) \Leftrightarrow(\delta(f, x) \wedge(x, y) \in f) \vee(\neg \delta(f, x) \wedge y=f),
$$

where $\delta(f, x):=(f$ is a function $\wedge x \in \operatorname{dom} f)$.

Theorem 21. Let $\varphi\left(x_{1}, \ldots, x_{n}\right)$ be a $\Sigma_{1}$ formula; let $\psi\left(x_{1}, \ldots, x_{n}\right)$ be a $\Delta_{1}$ formula; and let $\tau\left(x_{1}, \ldots, x_{n}\right)$ be a $\Delta_{1}$ term whose parameters are contained in the list $x_{1}, \ldots, x_{n}$. Then ZFC proves that, for every complete Boolean algebra $B$ and all $x_{1}, \ldots, x_{n}$,

(a) $\varphi\left(x_{1}, \ldots, x_{n}\right) \Rightarrow \mathbb{V}^{(B)} \vDash \varphi\left(x_{1}^{\wedge}, \ldots, x_{n}^{\wedge}\right)$;

(b) $\psi\left(x_{1}, \ldots, x_{n}\right) \Leftrightarrow \mathbb{V}^{(B)} \vDash \psi\left(x_{1}^{\wedge}, \ldots, x_{n}^{\wedge}\right)$;

(c) $\mathbb{V}^{(B)} \vDash\left(\tau\left(x_{1}, \ldots, x_{n}\right)^{\wedge}=\tau\left(x_{1}^{\wedge}, \ldots, x_{n}^{\wedge}\right)\right)$.

In particular, if $\tau$ is a $\Delta_{1}$ term without parameters, then $\mathrm{ZFC}$ proves $\mathbb{V}^{(B)} \vDash\left(\tau^{\wedge}=\tau\right)$.

Proof. A proof of (a) can be found in [4] (2.2.9) or [5] (4.2.9); (b) follows from (a); (c) is a synonym of the implication $y=\tau\left(x_{1}, \ldots, x_{n}\right) \Rightarrow \mathbb{V}^{(B)} \vDash\left(y^{\wedge}=\tau\left(x_{1}^{\wedge}, \ldots, x_{n}^{\wedge}\right)\right)$.

Proposition 26. Let $\tau\left(x_{1}, \ldots, x_{n}\right)$ be a term with parameters contained in the list $x_{1}, \ldots, x_{n}$. If the formula $x \in \tau\left(x_{1}, \ldots, x_{n}\right)$ is $\Sigma_{1}$ (with $x$ different from $x_{i}$ ), then ZFC proves that, for every complete Boolean algebra $B$ and all $x_{1}, \ldots, x_{n}$,

$$
\mathbb{V}^{(B)} \vDash\left(\tau\left(x_{1}, \ldots, x_{n}\right)^{\wedge} \subset \tau\left(x_{1}^{\wedge}, \ldots, x_{n}^{\wedge}\right)\right) .
$$

In particular, if $\tau$ has no parameters and the formula $x \in \tau$ is $\Sigma_{1}$, then $\mathbb{V}^{(B)} \vDash\left(\tau^{\wedge} \subset \tau\right)$.

Proof. If the containment $x \in \tau(\dddot{x})$ is $\Sigma_{1}$, then the formula $(\forall x \in y)(x \in \tau(\dddot{x}))$ is a $\Sigma_{1}$ definition for the inclusion $y \subset \tau(\ddot{x})$. Owing to Theorem 21 (a), we have $y \subset \tau(\ddot{x}) \Rightarrow$ $\mathbb{V}^{(B)} \vDash\left(y^{\wedge} \subset \tau\left(\dddot{x}^{\wedge}\right)\right)$, where $\dddot{x}^{\wedge}:=x_{1}^{\wedge}, \ldots, x_{n}^{\wedge}$. By substituting $\tau(\ddot{x})$ for $y$, we conclude that $\mathbb{V}^{(B)} \vDash\left(\tau(\ddot{x})^{\wedge} \subset \tau\left(\dddot{x}^{\wedge}\right)\right)$.

\subsection{Relative Classification of Terms}

In this subsection, we introduce the notion of relative $\Sigma_{1}$ definability for terms and demonstrate its logical connection with the canonical embedding in the Boolean-valued universe.

Definition 62. Let $\tau$ and $\dddot{\sigma}$ be arbitrary terms. Say that a formula $\varphi(T, \dddot{S})$ defines $\tau$ via $\dddot{\sigma}$ or, more exactly, defines $T=\tau$ via $\ddot{S}=\ddot{\sigma}$, if

$$
\mathrm{ZFC} \vdash(T=\tau \Leftrightarrow \varphi(T, \ddot{\sigma})) .
$$

Say that $\tau$ is $\Sigma_{1}$ definable via $\ddot{\sigma}$ and call $\tau$ a $\Sigma_{1}(\ddot{\sigma})$ term, if there is a $\Sigma_{1}$ formula that defines $\tau$ via $\ddot{\sigma}$. Say that terms $\tau$ and $\sigma$ are $\Sigma_{1}$ equivalent, if $\tau$ is $\Sigma_{1}(\sigma)$ and $\sigma$ is $\Sigma_{1}(\tau)$.

Proposition 27. Let $\tau, \dddot{\tau}, \dddot{\sigma}, \dddot{\rho}$ be terms.

(a) If $\tau$ is $\Delta_{1}$ then $\tau$ is $\Sigma_{1}(\ddot{\sigma})$.

(b) If $\tau$ is $\Sigma_{1}(\ddot{\sigma})$ and $\dddot{\sigma}$ are $\Delta_{1}$ then $\tau$ is $\Delta_{1}$. 
(c) If $\tau$ is $\Sigma_{1}(\ddot{\sigma}, \dddot{\rho})$ and $\dddot{\rho}$ are $\Sigma_{1}(\ddot{\sigma})$ then $\tau$ is $\Sigma_{1}(\ddot{\sigma})$.

(d) If $\tau$ is $\Sigma_{1}(\ddot{\sigma})$ and $\ddot{\sigma}$ are $\Sigma_{1}(\ddot{\rho})$ then $\tau$ is $\Sigma_{1}(\ddot{\rho})$.

(e) If $\tau\left(x_{1}, \ldots, x_{n}\right)$ and $\tau_{1}, \ldots, \tau_{n}$ are $\Sigma_{1}(\ddot{\sigma})$ then $\tau\left(\tau_{1}, \ldots, \tau_{n}\right)$ is $\Sigma_{1}(\ddot{\sigma})$.

(f) If $\tau(\ddot{x})$ is $\Delta_{1}$ then $\tau(\ddot{\sigma})$ is $\Sigma_{1}(\ddot{\sigma})$.

Proof. Assertion (a) is obvious.

(b): If a $\Sigma_{1}$ formula $\varphi\left(T, S_{1}, \ldots, S_{n}\right)$ defines $\tau$ via $\sigma_{1}, \ldots, \sigma_{n}$, and $\varphi_{i}\left(S_{i}\right)$ are $\Sigma_{1}$ definitions for $\sigma_{i}$, then $\tau$ admits the following $\Sigma_{1}$ definition:

$$
\left(\exists S_{1}, \ldots, S_{n}\right)\left(\varphi\left(T, S_{1}, \ldots, S_{n}\right) \wedge \varphi_{1}\left(S_{1}\right) \wedge \cdots \wedge \varphi_{n}\left(S_{n}\right)\right) .
$$

(c): If $\varphi\left(T, \dddot{S}, R_{1}, \ldots, R_{m}\right)$ defines $\tau$ via $\dddot{\sigma}, \rho_{1}, \ldots, \rho_{m}$, and $\psi_{j}\left(R_{j}, \dddot{S}\right)$ define $\rho_{j}$ via $\dddot{\sigma}$, then the following formula defines $\tau$ via $\dddot{\sigma}$ :

$$
\left(\exists R_{1}, \ldots, R_{m}\right)\left(\varphi\left(T, \dddot{S}, R_{1}, \ldots, R_{m}\right) \wedge \psi_{1}\left(R_{1}, \dddot{S}\right) \wedge \cdots \wedge \psi_{m}\left(R_{m}, \dddot{S}\right)\right) .
$$

(d): If $\varphi\left(T, S_{1}, \ldots, S_{n}\right)$ defines $\tau$ via $\sigma_{1}, \ldots, \sigma_{n}$, and $\varphi_{i}\left(S_{i}, \dddot{R}\right)$ define $\sigma_{i}$ via $\dddot{\rho}$, then the following formula defines $\tau$ via $\dddot{\rho}$ :

$$
\left(\exists S_{1}, \ldots, S_{n}\right)\left(\varphi\left(T, S_{1}, \ldots, S_{n}\right) \wedge \varphi_{1}\left(S_{1}, \dddot{R}\right) \wedge \cdots \wedge \varphi_{n}\left(S_{n}, \dddot{R}\right)\right) .
$$

(e): If $\varphi\left(T, \dddot{S}, x_{1}, \ldots, x_{n}\right)$ defines $T=\tau\left(x_{1}, \ldots, x_{n}\right)$ via $\dddot{S}=\dddot{\sigma}$, and $\varphi_{i}\left(T_{i}, \dddot{S}\right)$ define $T_{i}=\tau_{i}$ via $\ddot{S}=\ddot{\sigma}$, then the following formula defines $T=\tau\left(\tau_{1}, \ldots, \tau_{n}\right)$ via $\ddot{S}=\ddot{\sigma}$ :

$$
\left(\exists T_{1}, \ldots, T_{n}\right)\left(\varphi\left(T, \dddot{S}, T_{1}, \ldots, T_{n}\right) \wedge \varphi_{1}\left(T_{1}, \dddot{S}\right) \wedge \cdots \wedge \varphi_{n}\left(T_{n}, \dddot{S}\right)\right) .
$$

Assertion (f) is a particular case of (e).

Proposition 28. If $\tau(\ddot{x})$ is a $\Delta_{1}$ term with parameters $\dddot{x}, \varphi(\dddot{x}, \ddot{y})$ is a $\Delta_{1}$ formula, and $\dddot{\sigma}$ are arbitrary terms; then the term $\{\tau(\ddot{x}): \ddot{x} \in \ddot{\sigma} \wedge \varphi(\dddot{x}, \ddot{\sigma})\}$ is $\Sigma_{1}(\ddot{\sigma})$.

Proof. The claim follows from Propositions 25 and 27(f).

Lemma 35. Let $\tau(\ddot{x})$ be a $\Delta_{1}$ term with parameters $\ddot{x}$, and let $\varphi(\ddot{x}, y)$ be a $\Delta_{1}$ formula. Consider an arbitrary term $\sigma$ and put

$$
\rho=\{\tau(\dddot{x}): \dddot{x} \in \sigma \wedge \varphi(\dddot{x}, \sigma)\} .
$$

If $\pi$ is a $\Sigma_{1}(\sigma)$ term and $\mathrm{ZFC} \vdash(\pi \neq \varnothing)$, then the term $\rho^{\pi}$ is $\Sigma_{1}$ definable via $\sigma^{\pi}$. In particular, $\rho^{\omega}$ is $\Sigma_{1}$ definable via $\sigma^{\omega}$.

Proof. Since $\pi \neq \varnothing$ implies $\sigma=\left\{s(x): s \in \sigma^{\pi} \wedge x \in \pi\right\}$, the term $\sigma$ is $\Sigma_{1}\left(\sigma^{\pi}, \pi\right)$ by Proposition 28. Next, observe that $\pi$ is $\Sigma_{1}\left(\sigma^{\pi}\right)$. Indeed, if $\varphi(P, S)$ defines $P=\pi$ via $S=\sigma$ then, with account taken of $\mathrm{ZFC} \vdash(\pi \neq \varnothing)$, the formula

$$
(\mathcal{S}=\varnothing \wedge \varphi(P, \varnothing)) \vee(\exists s \in \mathcal{S})(P=\operatorname{dom} s)
$$

defines $P=\pi$ via $\mathcal{S}=\sigma^{\pi}$. From Proposition 27 (c) it follows that $\sigma$ is $\Sigma_{1}\left(\sigma^{\pi}\right)$. On the other hand, $\rho$ is $\Sigma_{1}(\sigma)$ by Proposition 28. According to Proposition 27(d), $\rho$ is $\Sigma_{1}\left(\sigma^{\pi}\right)$ and, consequently, there is a $\Sigma_{1}$ formula $\psi(R, \mathcal{S})$ such that ZFC $\vdash\left(R=\rho \Leftrightarrow \psi\left(R, \sigma^{\pi}\right)\right)$. Therefore, the following $\Sigma_{1}$ formula defines $\mathcal{R}=\rho^{\pi}$ via $\mathcal{S}=\sigma^{\pi}$ :

$$
\begin{aligned}
& (\exists R)(\psi(R, \mathcal{S}) \wedge(\forall r \in \mathcal{R})(r: \pi \rightarrow R)) \wedge \\
& \left(\forall s_{1}, \ldots, s_{n} \in \mathcal{S}\right)\left((\exists x \in \pi) \neg \varphi\left(s_{1}(x), \ldots, s_{n}(x), \mathcal{S}\right) \vee\right. \\
& \left.\quad(\exists r \in \mathcal{R})(\forall x \in \pi) r(x)=\tau\left(s_{1}(x), \ldots, s_{n}(x)\right)\right) .
\end{aligned}
$$


Definition 63. Given lists of terms $\ddot{\sigma}=\sigma_{1}, \ldots, \sigma_{m}$ and variables $\ddot{x}=x_{1}, \ldots, x_{n}$, introduce the notation

$$
\begin{gathered}
\dddot{\sigma}(\dddot{x}):=\sigma_{1}(\dddot{x}), \ldots, \sigma_{m}(\dddot{x}), \quad \dddot{x}^{\wedge}:=x_{1}^{\wedge}, \ldots, x_{n}^{\wedge} \\
\dddot{\sigma}(\dddot{x})^{\wedge}:=\sigma_{1}(\ddot{x})^{\wedge}, \ldots, \sigma_{m}(\ddot{x})^{\wedge}, \quad \ddot{\sigma}\left(\dddot{x}^{\wedge}\right):=\sigma_{1}\left(\ddot{x}^{\wedge}\right), \ldots, \sigma_{m}\left(\dddot{x}^{\wedge}\right)
\end{gathered}
$$

and agree to use the expression $\dddot{\sigma}(\dddot{x})^{\wedge}=\ddot{\sigma}\left(\dddot{x}^{\wedge}\right)$ as a shorthand for the formula

$$
\sigma_{1}(\dddot{x})^{\wedge}=\sigma_{1}\left(\dddot{x}^{\wedge}\right) \wedge \cdots \wedge \sigma_{m}(\ddot{x})^{\wedge}=\sigma_{m}\left(\dddot{x}^{\wedge}\right) .
$$

Theorem 22. Let $\sigma_{1}(\ddot{x}), \ldots, \sigma_{m}(\dddot{x})$ and $\tau(\ddot{x})$ be arbitrary terms whose parameters are contained in the list $\ddot{x}=x_{1}, \ldots, x_{n}$. If $\tau$ is $\Sigma_{1}$ definable via $\sigma_{1}, \ldots, \sigma_{m}$, then ZFC proves that, for every complete Boolean algebra $B$ and all $x_{1}, \ldots, x_{n}$,

$$
\mathbb{V}^{(B)} \vDash\left(\dddot{\sigma}(\dddot{x})^{\wedge}=\dddot{\sigma}\left(\dddot{x}^{\wedge}\right) \Rightarrow \tau(\dddot{x})^{\wedge}=\tau\left(\dddot{x}^{\wedge}\right)\right) .
$$

In particular, if $m=1$ and the term $\sigma:=\sigma_{1}$ has no parameters, then

$$
\mathbb{V}^{(B)} \vDash\left(\sigma^{\wedge}=\sigma \Rightarrow \tau(\dddot{x})^{\wedge}=\tau\left(\dddot{x}^{\wedge}\right)\right) ;
$$

and if both $\sigma$ and $\tau$ have no parameters, then

$$
\mathbb{V}^{(B)} \vDash\left(\sigma^{\wedge}=\sigma \Rightarrow \tau^{\wedge}=\tau\right) .
$$

Proof. Let $\varphi(T, \dddot{S}, \dddot{x})$ be a $\Sigma_{1}$ formula that defines $T=\tau(\ddot{x})$ via $\dddot{S}=\dddot{\sigma}(\dddot{x})$; that is, ZFC proves the formulas $(\forall \ddot{x}) \varphi(\tau(\ddot{x}), \ddot{\sigma}(\ddot{x}), \ddot{x})$ and $(\forall \ddot{x}, T)(\varphi(T, \ddot{\sigma}(\ddot{x}), \ddot{x}) \Rightarrow T=\tau(\ddot{x}))$. Due to the transfer principle, the two formulas are valid in $\mathbb{V}^{(B)}$. In particular, for all $\ddot{x}$, the following implication is valid in $\mathbb{V}^{(B)}$ :

$$
\varphi\left(\tau(\dddot{x})^{\wedge}, \dddot{\sigma}\left(\dddot{x}^{\wedge}\right), \dddot{x}^{\wedge}\right) \Rightarrow \tau(\dddot{x})^{\wedge}=\tau\left(\dddot{x}^{\wedge}\right) .
$$

According to Theorem $21(\mathrm{a}), \varphi(\tau(\ddot{x}), \dddot{\sigma}(\ddot{x}), \dddot{x})$ implies the validity in $\mathbb{V}^{(B)}$ of the formula

$$
\varphi\left(\tau(\dddot{x})^{\wedge}, \ddot{\sigma}(\dddot{x})^{\wedge}, \dddot{x}^{\wedge}\right) .
$$

It remains to observe that $(42),(43)$ and $\dddot{\sigma}(\dddot{x})^{\wedge}=\dddot{\sigma}\left(\dddot{x}^{\wedge}\right)$ imply $\tau(\dddot{x})^{\wedge}=\tau\left(\dddot{x}^{\wedge}\right)$.

\subsection{Embedding the Reals in the Boolean-Valued Universe}

As was noted in Remark 6, the field of reals in the Boolean-valued universe is often defined as an arbitrary element $\mathcal{R} \in \mathbb{V}^{(B)}$ that is a Dedekind-complete totally ordered field inside $\mathbb{V}^{(B)}$. However, the choice of such an $\mathcal{R}$ is usually restricted by the agreement that the assertion " $\mathbb{R}^{\wedge}$ is a subfield of $\mathcal{R}^{\prime}$ " should be valid in $\mathbb{V}^{(B)}$; see, e.g., [4] (5.2.2), [5] (10.3.3). Practically, this means that the field of reals in $\mathbb{V}^{(B)}$ is declared to be an arbitrary Dedekind completion of the Archimedean totally ordered field $\mathbb{R}^{\wedge}$.

The above-described introduction of the symbol $\mathcal{R}$ is represented by a conservative extension of set theory and is therefore logically flawless; however, it is technically inconvenient because of the lack of elimination (see Remark 6). There is also a methodical demerit: in the above approach, the definitions of the reals in ZFC and inside $\mathbb{V}^{(B)}$ occur different. Moreover, the notion of real number in the Boolean-valued universe fails to be "internal," since it employs an object, $\mathbb{R}^{\wedge}$, formally undefinable inside $\mathbb{V}^{(B)}$.

Within the formalism described in Remark 7, the field of reals is defined by an eliminable extension, the constant $\mathbb{R}$ becomes universal, and the notion of real number occurs the same for all models of set theory. Nevertheless, while eliminating the above-mentioned technical and methodical "defects," we run the risk of losing the conventional condition $\mathbb{V}^{(B)} \vDash\left(\underline{\mathbb{R}^{\wedge}} \subset \mathbb{R}\right)$.

The following question seems relevant: Which complete Boolean algebras $B$ and

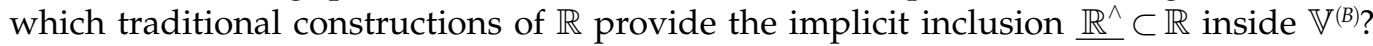
As an illustrative example of the technique presented in Sections 5.1 and 5.2, we will 
study the question and show that the relation $\mathbb{V}^{(B)} \vDash\left(\underline{\mathbb{R}^{\wedge}} \subset \mathbb{R}\right)$ always holds if the reals are defined as Dedekind cuts (see Theorem 23), whereas Cantor's approach, based on the Cauchy sequences of rationals, provides the inclusion only in the case of a $\sigma$-distributive Boolean algebra $B$ (see Proposition 29). The main tool will be the notion of $\Delta_{1}$ term.

Continuing the tradition, we hide the syntax of outer terms (see Definition 19) and, in particular, write $\mathbb{V}^{(B)} \vDash\left(\mathbb{R}^{\wedge} \subset \mathbb{R}\right)$ instead of $\mathbb{V}^{(B)} \vDash\left(\underline{\mathbb{R}^{\wedge}} \subset \mathbb{R}\right)$.

Remark 19. The information presented in Section 5.1 provides a convenient tool that helps to successively construct expressions within the classes $\Delta_{0}, \Sigma_{1}$, and $\Delta_{1}$. In particular, by employing the tool, we can easily verify that all the components of the conventionally defined number systems $\left(\omega,+_{\omega}, \cdot \omega_{\omega}, \leqslant_{\omega}\right),\left(\mathbb{Z},+_{\mathbb{Z}^{\prime}} \cdot \cdot_{\mathbb{Z}}, \leqslant_{\mathbb{Z}}\right)$, and $\left(\mathbb{Q},+_{\mathbb{Q}^{\prime}} \cdot \mathbb{Q}^{\prime} \leqslant \mathbb{Q}_{\mathbb{Q}}\right)$ are $\Delta_{1}$ terms. For instance,

$$
\mathbb{Z}=\{m-n: m, n \in \omega\}
$$

where $m-n=\left\{\left(m^{\prime}, n^{\prime}\right): m^{\prime}, n^{\prime} \in \omega, m+{ }_{\omega} n^{\prime}=m^{\prime}+{ }_{\omega} n\right\}$;

$$
\mathbb{Q}=\left\{i / j: i, j \in \mathbb{Z}, j \neq 0_{\mathbb{Z}}\right\},
$$

where $i / j=\left\{\left(i^{\prime}, j^{\prime}\right): i^{\prime}, j^{\prime} \in \mathbb{Z}, j^{\prime} \neq 0_{\mathbb{Z}}, i \cdot_{\mathbb{Z}} j^{\prime}=i^{\prime} \cdot{ }_{\mathbb{Z}} j\right\}$; the operation ${ }_{\omega}$ admits the $\Sigma_{1}$ definition

$$
+_{\omega}: \omega^{2} \rightarrow \omega \wedge(\forall m, n \in \omega)\left(m+_{\omega} 0=m \wedge m+_{\omega}(n+1)=\left(m+{ }_{\omega} n\right)+1\right) ;
$$

the relation $\leqslant_{\mathbb{Z}}$ is the $\Delta_{1}$ term

$$
\leqslant_{\mathbb{Z}}=\left\{\left(m-n, m^{\prime}-n^{\prime}\right): m, n, m^{\prime}, n^{\prime} \in \omega, m+_{\omega} n^{\prime} \leqslant_{\omega} m^{\prime}+{ }_{\omega} n\right\} .
$$

Consequently, due to Theorem 21, for each complete Boolean algebra $B$, the following equalities are valid in $\mathbb{V}^{(B)}$.

$$
\begin{aligned}
& \left(\omega^{\wedge},+_{\omega}^{\wedge}, \cdot \hat{\omega}, \leqslant_{\omega}^{\wedge}\right)=\left(\omega,+_{\omega}, \cdot{ }_{\omega},{ }_{\omega}\right), \\
& \left(\mathbb{Z}^{\wedge},+_{\mathbb{Z}}^{\wedge}, \bullet_{\mathbb{Z}}^{\wedge}, \leqslant_{\mathbb{Z}}^{\wedge}\right)=\left(\mathbb{Z},+_{\mathbb{Z}^{\prime}} \cdot \bullet_{\mathbb{Z}}, \leqslant_{\mathbb{Z}}\right), \\
& \left(\mathbb{Q}^{\wedge},+_{\mathbb{Q}}^{\wedge}, \hat{\mathbb{Q}}^{\prime} \leqslant \hat{\mathbb{Q}}\right)=\left(\mathbb{Q},+_{\mathbb{Q}^{\prime}} \cdot \cdot_{\mathbb{Q}}, \leqslant \mathbb{Q}\right) \text {. }
\end{aligned}
$$

Definition 64. Recall that a Dedekind cut is a nonempty downwards closed subset $x \subset \mathbb{Q}$ that differs from $\mathbb{Q}$ and has no greatest element. The order and addition on the set $\mathbb{R}_{\mathrm{D}}$ of all Dedekind cuts are defined by the axioms $x \leqslant y \Leftrightarrow x \subset y, x+y=\left\{p+_{\mathbb{Q}} q: p \in x, q \in y\right\}$. To define the multiplication, we first introduce the notion of positive cut: $0 \leqslant x \Leftrightarrow \mathbb{Q}^{-} \subset x$, where $\mathbb{Q}^{-}=\left\{q \in \mathbb{Q}: q<_{\mathbb{Q}} 0_{\mathbb{Q}}\right\} ;$ next, define the unary negation: $-x=\left\{p-{ }_{\mathbb{Q}} q: p \in \mathbb{Q}^{-}, q \in \mathbb{Q} \backslash x\right\} ;$ then introduce the multiplication $x \cdot y=\mathbb{Q}^{-} \cup\left\{p \cdot \mathbb{Q} q: p \in x, q \in y, p, q \geqslant \mathbb{Q}_{\mathbb{Q}} 0_{\mathbb{Q}}\right\}$ for positive cuts; and, finally, define the product $x \cdot y$ for the rest pairs of $x$ and $y$ as $-(-x \cdot y),-(x \cdot-y)$, or $-x \cdot-y$, depending on which of the two cuts are not positive.

Theorem 23. For each complete Boolean algebra $B$, the inclusion $\mathbb{R}_{\mathrm{D}}^{\wedge} \subset \mathbb{R}_{\mathrm{D}}$ is valid in $\mathbb{V}^{(B)}$. Moreover, $\left(\mathbb{R}_{\mathrm{D}}^{\wedge},+^{\wedge}, \cdot^{\wedge}, \leqslant^{\wedge}\right)$ is an ordered subfield of $\left(\mathbb{R}_{\mathrm{D}},+, \cdot, \leqslant\right)$ inside $\mathbb{V}^{(B)}$.

Proof. Since the containment $x \in \mathbb{R}_{\mathrm{D}}$ is equivalent to the $\Sigma_{1}$ (and even $\Delta_{1}$ ) formula

$$
\begin{aligned}
& x \neq \varnothing \wedge x \subset \mathbb{Q} \wedge x \neq \mathbb{Q} \wedge \\
& (\forall q \in x)(\forall p \in \mathbb{Q})\left(p \leqslant_{\mathbb{Q}} q \Rightarrow p \in x\right) \wedge(\forall q \in x)(\exists p \in x)\left(q<_{\mathbb{Q}} p\right),
\end{aligned}
$$

by Proposition 26 we have $\mathbb{V}^{(B)} \vDash\left(\mathbb{R}_{\mathrm{D}}^{\wedge} \subset \mathbb{R}_{\mathrm{D}}\right)$.

From the definitions of the addition, multiplication, and order on $\mathbb{R}_{\mathrm{D}}$, it is clear that the terms $x+y, x \cdot y$ and the formula $x \leqslant y$ are $\Delta_{1}$. Consequently, the containment $z \in+$ admits the $\Sigma_{1}$ definition

$$
(\exists x, y)\left(x, y \in \mathbb{R}_{\mathrm{D}} \wedge z=(x, y, x+y)\right),
$$


and, therefore, $\mathbb{V}^{(B)} \vDash\left(+^{\wedge} \subset+\right)$ by Proposition 26. With account taken of the validity $\mathbb{V}^{(B)} \models\left(+^{\wedge}: \mathbb{R}_{\mathrm{D}}^{\wedge} \times \mathbb{R}_{\mathrm{D}}^{\wedge} \rightarrow \mathbb{R}_{\mathrm{D}}^{\wedge}\right)$, we thus conclude that $\mathbb{V}^{(B)} \models\left(+^{\wedge}=+\left.\right|_{\mathbb{R}_{\mathrm{D}}^{\wedge} \times \mathbb{R}_{\mathrm{D}}^{\wedge}}\right)$. Similar arguments justify the relations $\mathbb{V}^{(B)} \models\left(\cdot^{\wedge}=\left.\cdot\right|_{\mathbb{R}_{\mathrm{D}}^{\wedge} \times \mathbb{R}_{\mathrm{D}}^{\wedge}}\right)$ and $\mathbb{V}^{(B)} \models\left(\leqslant^{\wedge}=\leqslant \cap\left(\mathbb{R}_{\mathrm{D}}^{\wedge} \times \mathbb{R}_{\mathrm{D}}^{\wedge}\right)\right)$.

Remark 20. In connection with the above proof, it is worth noting that, actually, the membership of the terms $x+y$ and $x \cdot y$ in class $\Delta_{1}$ does not require a verification. Regardless of what formulas those terms are defined by, the corresponding operations + and $\cdot$ should be continuous extension onto $\mathbb{R}_{\mathrm{D}} \times \mathbb{R}_{\mathrm{D}}$ of the respective operations $+_{\mathbb{Q}}$ and $\cdot_{\mathbb{Q}}$ having been transferred from the field $\mathbb{Q}$ onto the subfield $\left[[\mathbb{Q}] \subset \mathbb{R}_{\mathrm{D}}\right.$ by means of the isomorphic embedding

$$
q \in \mathbb{Q} \mapsto \imath(q)=\left\{p \in \mathbb{Q}: p<_{\mathbb{Q}} q\right\} \in \mathbb{R}_{\mathrm{D}} .
$$

Since the term $\imath(q)$ and the relation $x \leqslant y$ are $\Delta_{1}$, the term $x+y$ admits the $\Sigma_{1}$ definition

$$
\begin{aligned}
z=x+y & \Leftrightarrow\left(\imath\left(p++_{\mathbb{Q}} q\right) \rightarrow z \text { as } p \rightarrow x, q \rightarrow y\right) \\
& \Leftrightarrow\left(\forall \varepsilon \in \mathbb{Q}^{+}\right)\left(\exists \delta \in \mathbb{Q}^{+}\right)(\forall p, q \in \mathbb{Q})\left(p \in B(x, \delta), q \in B(y, \delta) \Rightarrow p++_{\mathbb{Q}} q \in B(z, \varepsilon)\right),
\end{aligned}
$$

where $p \in B(x, \delta) \Leftrightarrow \imath\left(p-{ }_{\mathbb{Q}} \delta\right) \leqslant x \leqslant \imath\left(p+{ }_{\mathbb{Q}} \delta\right)$. The same is true of the term $x \cdot y$.

Similar arguments, that lead to Theorem 23, are valid for other classical constructions of the field of reals, including those based on infinite decimal fractions (or records in other positional numeral systems) and continued fractions. Within those approaches, as in the case of Dedekind cuts, the formulas $x \in \mathbb{R}$ and $x \leqslant y$ are $\Delta_{1}$ and there is an embedding $\imath: \mathbb{Q} \rightarrow \mathbb{R}$ by means of a $\Delta_{1}$ term $\imath(q)$.

Definition 65. A complete Boolean algebra B is called $\sigma$-distributive or, which is the same, $(\omega, 2)$ distributive or $(\omega, \omega)$-distributive (see [8] (Section 14)), if it satisfies each of the following equivalent conditions (see [11] (Section 19), [13]):

(a) $\bigwedge_{i \in \omega} \bigvee_{j \in \omega} b(i, j)=\bigvee_{j \in \omega} \bigwedge_{i \in \omega} b(i, j(i))$ for all $b: \omega^{2} \rightarrow B$;

(b) $\bigvee_{i \in \omega} \bigwedge_{j \in \omega} b(i, j)=\bigwedge_{j \in \omega^{\omega}} \bigvee_{i \in \omega} b(i, j(i))$ for all $b: \omega^{2} \rightarrow B$;

(c) $\bigvee_{s \in\{1,-1\} \omega} \bigwedge_{i \in \omega} s(i) b(i)=1_{B}$ for all $b: \omega \rightarrow B$;

(d) from each sequence of countable (or finite, or two-element) covers of $B$ we can refine a cover;

(e) from each sequence of countable (or finite, or two-element) partitions of $B$ we can refine a partition.

The Boolean algebra of cosets of Lebesgue measurable subsets of $\mathbb{R}$ is not $\sigma$-distributive. Every atomic complete Boolean algebra is $\sigma$-distributive. The completion of the quotient Boolean algebra $\mathcal{P}(\omega) / \mathcal{P}_{\text {fin }}(\omega)$ serves as an example of an atomless $\sigma$-distributive complete Boolean algebra; see [13].

Theorem 24. The following properties of a complete Boolean algebra $B$ are equivalent:

(a) $B$ is $\sigma$-distributive;

(b) $\mathbb{V}^{(B)} \vDash\left(\mathcal{P}(\omega)^{\wedge}=\mathcal{P}(\omega)\right)$;

(c) $\mathbb{V}^{(B)} \vDash\left(\left(\omega^{\omega}\right)^{\wedge}=\omega^{\omega}\right)$;

(d) $\mathbb{V}^{(B)} \vDash\left(\mathbb{R}_{\mathrm{D}}^{\wedge}=\mathbb{R}_{\mathrm{D}}\right)$;

(e) $\mathbb{V}^{(B)} \models$ (the ordered fields $\mathbb{R}^{\wedge}$ and $\mathbb{R}$ are isomorphic), where $\mathbb{R}$ is the ordered field of reals introduced by any of the conventional definitions.

Proof. The implications (a) $\Leftrightarrow$ (b) $\Leftrightarrow$ (c) are well known; see [3] $(2.14,2.15)$. The equivalence (d) $\Leftrightarrow$ (e) is easily established with the help of Theorem 23 , the denseness of $\mathbb{R}_{\mathrm{D}}^{\wedge}$ in $\mathbb{R}_{\mathrm{D}}$, and the membership in class $\Sigma_{1}$ of the assertion on isomorphism of two ordered fields. A proof of $(\mathrm{a}) \Leftrightarrow(\mathrm{e})$ can be found in [13].

Each of the conditions (a)-(e) is equivalent to the assertion that the Kantorovich space with base $B$ is one-dimensional. This fact is used in [13] for proving the equivalence (a) $\Leftrightarrow($ e) by means of the theory of ordered vector spaces. Nevertheless, from Theorem 22 
it is clear that the equivalence (b) $\Leftrightarrow(\mathrm{c}) \Leftrightarrow(\mathrm{d})$ admits a "syntactic" reason: the terms $\mathcal{P}(\omega)$, $\omega^{\omega}$, and $\mathbb{R}_{\mathrm{D}}$ are $\Sigma_{1}$ equivalent. (The corresponding mutual $\Sigma_{1}$ definitions can be formulated with the use of, for instance, the representation of the reals as infinite binary fractions or continued fractions. In this approach, an essential role is played by the fact that the formula $x \in \mathbb{R}_{\mathrm{D}}$ is $\Delta_{1}$.) Observe also that the list $\mathcal{P}(\omega), \omega^{\omega}, \mathbb{R}_{\mathrm{D}}$ can be complemented by, for instance, the terms $\mathbb{Z}^{\omega}$ and $\mathbb{Q}^{\omega}$, since the latter are $\Sigma_{1}$ equivalent to $\omega^{\omega}$ due to Lemma 35 and the representations $\mathbb{Z}=\{n-m: n, m \in \omega\}, \mathbb{Q}=\left\{i / j: i, j \in \mathbb{Z}, j \neq 0_{\mathbb{Z}}\right\}$, $\omega=\left\{\lfloor q\rfloor: q \in \mathbb{Q}, q \geqslant \mathbb{Q} 0_{\mathbb{Q}}\right\}$.

Definition 66. Cantor's approach to the definition of the reals starts with considering the set

$$
\mathbb{Q}_{\mathrm{C}}^{\omega}=\left\{s \in \mathbb{Q}^{\omega}:\left(\forall q \in \mathbb{Q}^{+}\right)(\exists m \in \omega)(\forall n \in \omega)\left(m \leqslant_{\omega} n \Rightarrow\left|s(m)-{ }_{\mathbb{Q}} s(n)\right| \leqslant_{\mathbb{Q}} q\right)\right\}
$$

of all Cauchy sequences of rational numbers. The set $\mathbb{R}_{C}$ of reals is then defined as the quotient $\mathbb{Q}_{\mathrm{C}}^{\omega} / \sim$ with respect to the equivalence

$$
s \sim t \Leftrightarrow\left(\forall q \in \mathbb{Q}^{+}\right)(\exists m \in \omega)(\forall n \in \omega)\left(m \leqslant_{\omega} n \Rightarrow\left|s(n)-{ }_{\mathbb{Q}} t(n)\right| \leqslant_{\mathbb{Q}} q\right)
$$

end endowed with the natural addition, multiplication and order.

As is shown by the following proposition, in Cantor's approach, the logical structure of the reals is considerably more complex than that of Dedekind cuts, and the inclusion $\mathbb{R}^{\wedge} \subset \mathbb{R}$ is not valid in all Boolean-valued models $\mathbb{V}^{(B)}$.

Proposition 29. The inclusion $\mathbb{R}_{C}^{\wedge} \subset \mathbb{R}_{C}$ is valid in $\mathbb{V}^{(B)}$ if and only if the complete Boolean algebra $B$ is $\sigma$-distributive.

Proof. Suppose that $\mathbb{V}^{(B)} \vDash\left(\mathbb{R}_{\mathrm{C}}^{\wedge} \subset \mathbb{R}_{\mathrm{C}}\right)$.

Show first that $\mathbb{V}^{(B)} \vDash\left(0_{C}^{\wedge}=0_{C}\right)$, where $0_{C} \in \mathbb{R}_{C}$ is the set of all vanishing sequences of rational numbers. Indeed, $\mathbb{V}^{(B)} \vDash\left(\mathbb{R}_{\mathrm{C}}^{\wedge} \subset \mathbb{R}_{\mathrm{C}}\right)$ implies $\mathbb{V}^{(B)} \vDash\left(0_{\mathrm{C}}^{\wedge} \in \mathbb{R}_{\mathrm{C}}\right)$. Since the formula $s \in 0_{\mathrm{C}}$ admits the $\Sigma_{1}$ definition

$$
s: \omega \rightarrow \mathbb{Q} \wedge\left(\forall q \in \mathbb{Q}^{+}\right)(\exists m \in \omega)(\forall n \in \omega)\left(m \leqslant_{\omega} n \Rightarrow|s(n)| \leqslant_{\mathbb{Q}} q\right),
$$

by Proposition 26 we have $\mathbb{V}^{(B)} \vDash\left(0_{\mathrm{C}}^{\wedge} \subset 0_{\mathrm{C}}\right)$. Consequently, inside $\mathbb{V}^{(B)}$, the elements $0_{\mathrm{C}}^{\wedge}$ and $0_{\mathrm{C}}$ of the quotient $\mathbb{R}_{\mathrm{C}}=\mathbb{Q}_{\mathrm{C}}^{\omega} / \sim$ satisfy the inclusion $0_{\mathrm{C}}^{\wedge} \subset 0_{\mathrm{C}}$ and therefore coincide: $\mathbb{V}^{(B)} \vDash\left(0_{\mathrm{C}}^{\wedge}=0_{\mathrm{C}}\right)$.

Observe that $\mathcal{P}(\omega)=\left\{\operatorname{supp}(s): s \in 0_{\mathrm{C}}\right\}$, where $\operatorname{supp}(s)=\left\{n \in \omega: s(n) \neq 0_{\mathbb{Q}}\right\}$, and so, due to Propositions 25 and 28, the term $\mathcal{P}(\omega)$ is $\Sigma_{1}$ definable via $0_{\text {C }}$. By Theorem 22, $\mathbb{V}^{(B)} \vDash\left(0_{\mathrm{C}}^{\wedge}=0_{\mathrm{C}}\right)$ implies $\mathbb{V}^{(B)} \vDash\left(\mathcal{P}(\omega)^{\wedge}=\mathcal{P}(\omega)\right)$; therefore, $B$ is $\sigma$-distributive according to Theorem 24.

Conversely, let $B$ be $\sigma$-distributive. Then $\mathbb{V}^{(B)} \vDash\left(\left(\mathbb{Q}^{\omega}\right)^{\wedge}=\mathbb{Q}^{\omega}\right)$ (see Theorem 24). Given a sequence $s \in \mathbb{Q}_{\mathrm{C}}^{\omega}$, consider the coset $\rho(s) \in \mathbb{R}_{\mathrm{C}}$. With account taken of Proposition 24(d), from (44) it is clear that the formula $s \sim t$ is $\Delta_{1}$. According to Proposition 28, the term $\rho(s)=\left\{t: t \in \mathbb{Q}^{\omega}, s \sim t\right\}$ is $\Sigma_{1}$ definable via $\mathbb{Q}^{\omega}$. By Theorem $22, \mathbb{V}^{(B)} \vDash\left(\left(\mathbb{Q}^{\omega}\right)^{\wedge}=\mathbb{Q}^{\omega}\right)$ implies $\mathbb{V}^{(B)} \vDash\left(\rho(s)^{\wedge}=\rho\left(s^{\wedge}\right)\right)$ for all $s$. Consequently, $\left(\forall s \in \mathbb{Q}_{C}^{\omega}\right) \mathbb{V}^{(B)} \vDash\left(\rho(s)^{\wedge} \in \mathbb{R}_{\mathrm{C}}\right)$, that is, $\left(\forall x \in \mathbb{R}_{\mathrm{C}}\right) \mathbb{V}^{(B)} \vDash\left(x^{\wedge} \in \mathbb{R}_{\mathrm{C}}\right)$, and so $\mathbb{V}^{(B)} \vDash\left(\mathbb{R}_{\mathrm{C}}^{\wedge} \subset \mathbb{R}_{\mathrm{C}}\right)$.

\subsection{Further Applications}

As another application of the Lévy hierarchy, we present a short justification for the Boolean-valued validity of the equality $\mathcal{P}_{\text {fin }}(X)^{\wedge}=\mathcal{P}_{\text {fin }}\left(X^{\wedge}\right)$ and show that the term $\{x \in X: \varphi(x)\}$ can fail to be $\Sigma_{1}$ even in the case of a $\Sigma_{1}$ formula $\varphi$.

As is known (see [4] (3.1.10, 3.1.11), [5] $(5.1 .8,5.1 .9))$, given a complete Boolean algebra $B$ and an arbitrary set $X$, the equality

$$
\mathcal{P}_{\text {fin }}(X)^{\wedge}=\mathcal{P}_{\text {fin }}\left(X^{\wedge}\right)
$$

is valid in $\mathbb{V}^{(B)}$, which fact can be verified by a tedious calculation of the Boolean truth value. As an illustration of the technique of $\Delta_{1}$ terms, we will demonstrate that the above validity 
is a direct consequence of Theorem 21 (c) and admits a "syntactic" reason, the membership of the term $\mathcal{P}_{\text {fin }}(X)$ in class $\Delta_{1}$.

Indeed, given a set $X$, consider the function $\mathbb{F}_{X}: \omega \rightarrow \mathcal{P}(X)$ that maps each number $n \in \omega$ to the totality $\mathbb{F}_{X}(n)$ of all subsets of $X$ consisting of $n$ elements. It remains to observe that $\mathcal{P}_{\text {fin }}(X)=\cup\left(\operatorname{im} \mathbb{F}_{X}\right)$ and the term $\mathbb{F}_{X}$ admits the $\Sigma_{1}$ definition

$$
\begin{aligned}
& \mathbb{F}_{X} \text { is a function } \wedge \operatorname{dom} \mathbb{F}_{X}=\omega \wedge \mathbb{F}_{X}(0)=\{\varnothing\} \wedge \\
& (\forall n \in \omega) \mathbb{F}_{X}(n+1)=\left\{y \cup\{x\}: y \in \mathbb{F}_{X}(n), x \in X, x \notin y\right\} .
\end{aligned}
$$

We conclude the article with an example that shows that Proposition 25, valid for $\Delta_{1}$ formulas, cannot be expanded to $\Sigma_{1}$ formulas.

Example 6. The formula $\varphi(x):=(\mathcal{P}(\omega) \not \subset x)$ is $\Sigma_{1}$, while, if ZFC is consistent, the term $\{x \in X: \varphi(x)\}$ is not $\Sigma_{1}$.

Proof. For brevity, introduce the symbol $\mathbb{P}:=\mathcal{P}(\omega)$. The formula $\mathbb{P} \not \subset x$ is $\Sigma_{1}$, since $\mathbb{P} \not \subset x \Leftrightarrow(\exists y)(y \subset \omega \wedge y \notin x)$. Assume that ZFC is consistent, and show that the term

$$
\tau(X):=\{x \in X: \mathbb{P} \not \subset x\}
$$

is not $\Sigma_{1}$. According to Proposition 24(b) and Theorem 21 (c), it suffices to find a complete Boolean algebra $B$ such that $\tau(\{\mathbb{P}\})^{\wedge} \neq \tau\left(\{\mathbb{P}\}^{\wedge}\right)$ inside $\mathbb{V}^{(B)}$.

Let $A$ be an arbitrary complete Boolean algebra that is not $\sigma$-distributive. From Theorem 24 it follows that $\mathbb{V}^{(A)} \not \models\left(\mathbb{P}^{\wedge}=\mathbb{P}\right)$, that is, the truth value $a=\left[\mathbb{P}^{\wedge} \neq \mathbb{P}\right]_{\mathbb{V}(A)} \in A$ is nonzero. Then, by considering the component $B=[0, a] \subset A$, we obtain a complete Boolean algebra $B$ such that $\mathbb{P}^{\wedge} \neq \mathbb{P}$ inside $\mathbb{V}^{(B)}$.

Since $x \in \mathbb{P} \Leftrightarrow(\forall y \in x)(y \in \omega)$, the formula $x \in \mathbb{P}$ is $\Sigma_{1}$. According to Proposition 26, inside $\mathbb{V}^{(B)}$ we have $\mathbb{P}^{\wedge} \subset \mathbb{P}$, which, due to the inequality $\mathbb{P}^{\wedge} \neq \mathbb{P}$, implies $\mathbb{P} \not \subset \mathbb{P}^{\wedge}$ and, therefore,

$$
\tau\left(\{\mathbb{P}\}^{\wedge}\right)=\tau\left(\left\{\mathbb{P}^{\wedge}\right\}\right)=\left\{x \in\left\{\mathbb{P}^{\wedge}\right\}: \mathbb{P} \not \subset x\right\}=\left\{\mathbb{P}^{\wedge}\right\} .
$$

On the other hand,

$$
\tau(\{\mathbb{P}\})^{\wedge}=\{x \in\{\mathbb{P}\}: \mathbb{P} \not \subset x\}^{\wedge}=\varnothing^{\wedge}=\varnothing .
$$

The above example implies in particular that the assertion in [4] (3.1.3) and [5] (5.1.3) is valid only for the case in which the formula $\varphi$ is $\Delta_{1}$.

\section{Conclusions}

Despite the volume of the present paper, this is a research article rather than a survey. The information presented here can be divided into three categories-common knowledge, previously known facts, and new material. The first category includes Subsections 2.1, 2.4 and, partially, 2.5. The rest of Section 2 can be attributed to common knowledge as well, although the exposition is different from what can be found elsewhere. Previously known facts take a small portion of the total volume and are always supplied with references.

References are also provided for the author's publications, with the following exception. The present article continues, develops, and supersedes the study initiated in [15-17]. The content of those three papers has been redesigned, corrected, extended, and distributed across the new subsections. It would be difficult to label numerous small fragments with proper references, and there is little point in doing that. (Moreover, [15] is available only in Russian.) Therefore, we confine ourselves to including [15-17] in the list of references.

One-level continuous numbering of subsections is not the best choice in the case of a deeply structured article with numerous cross-references. A table of contents could also make it easier to navigate through the text. Since the journal's rules do not include changing the numbering style or adding a table of contents, we made the author's version of this paper available as Supplementary Materials (see the link below).

The author is indebted to Professor Robert M. Solovay for a fruitful discussion that became the main motivation for the present research. 
Supplementary Materials: The author's version of this article (with a table of contents and different section numbering style) is available online at https:/ / www.mdpi.com/article/10.3390/math90910 $56 /$ s1.

Funding: The work was carried out in the framework of the State Task to the Sobolev Institute of Mathematics (Project 0314-2019-0005).

Institutional Review Board Statement: Not applicable.

Informed Consent Statement: Not applicable.

Conflicts of Interest: The author declares no conflict of interest.

\section{References}

1. Solovay, R.M.; Tennenbaum, S. Iterated Cohen extensions and Souslin's problem. Ann. Math. 1971, 94, 201-245. [CrossRef]

2. Jech, T. Set Theory, The Third Millennium ed.; Revised and Expanded; Springer: Berlin/Heidelberg, Germany, 2003.

3. Bell, J.L. Set Theory. Boolean-Valued Models and Independence Proofs; Clarendon Press: New York, NY, USA, 2005.

4. Kusraev, A.G.; Kutateladze, S.S. Boolean Valued Analysis; Kluwer: Dordrecht, The Netherlands, 1999.

5. Kusraev, A.G.; Kutateladze, S.S. Introduction to Boolean Valued Analysis; Nauka: Moscow, Russia, 2005.

6. Solovay, R.M. (Department of Mathematics, University of California, Berkeley, CA, USA). Private communication, 2005.

7. Boolos, G.; Burgess, J.P.; Jeffrey, R.C. Computability and Logic, 5th ed.; Cambridge University Press: Cambridge, UK, 2007.

8. Koppelberg, S. Handbook of Boolean Algebras; North-Holland: Amsterdam, The Netherlands, 1989; Volume 1.

9. Gutman, A.E.; Losenkov, G.A. Function representation of the Boolean-valued universe. Sib. Adv. Math. 1998, 8, 99-120.

10. Lévy, A. A Hierarchy of Formulas in Set Theory; American Mathematical Society: Providence, RI, USA, 1965.

11. Sikorski, R. Boolean Algebras, 3rd ed.; Springer: Berlin/Heidelberg, Germany, 1969.

12. Jech, T. Distributive Laws. In Handbook of Boolean Algebras; North-Holland: Amsterdam, The Netherlands; London, UK, 1989; Chapter 8; pp. 317-331.

13. Gutman, A.E. Locally one-dimensional $K$-spaces and $\sigma$-distributive Boolean algebras. Sib. Adv. Math. 1995, 5, 42-48.

14. Bourbaki, N. Theory of Sets; Springer: Berlin/Heidelberg, Germany, 2004.

15. Gutman, A.E. An Example of Using $\Delta_{1}$ Terms in Boolean-Valued Analysis; Vladikavkaz. Mat. Zh. 2012, 14, 47-63. [CrossRef]

16. Gutman, A.E. Boolean-valued universe as an algebraic system. I. Sib. Math. J. 2019, 60, 810-827. [CrossRef]

17. Gutman, A.E. Boolean-valued universe as an algebraic system. II. Sib. Math. J. 2020, 61, 426-452. [CrossRef] 